\title{
Vascular dysfunction : at the heart of cardiovascular disease, cognitive impairment and depressive symptoms
}

Citation for published version (APA):

van Sloten, T. T. (2015). Vascular dysfunction : at the heart of cardiovascular disease, cognitive impairment and depressive symptoms. [Doctoral Thesis, Maastricht University]. Maastricht University. https://doi.org/10.26481/dis.20150520ts

Document status and date:

Published: 01/01/2015

DOI:

10.26481/dis.20150520ts

Document Version:

Publisher's PDF, also known as Version of record

Please check the document version of this publication:

- A submitted manuscript is the version of the article upon submission and before peer-review. There can be important differences between the submitted version and the official published version of record.

People interested in the research are advised to contact the author for the final version of the publication, or visit the DOI to the publisher's website.

- The final author version and the galley proof are versions of the publication after peer review.

- The final published version features the final layout of the paper including the volume, issue and page numbers.

Link to publication

\footnotetext{
General rights rights.

- You may freely distribute the URL identifying the publication in the public portal. please follow below link for the End User Agreement:

www.umlib.nl/taverne-license

Take down policy

If you believe that this document breaches copyright please contact us at:

repository@maastrichtuniversity.nl

providing details and we will investigate your claim.
}

Copyright and moral rights for the publications made accessible in the public portal are retained by the authors and/or other copyright owners and it is a condition of accessing publications that users recognise and abide by the legal requirements associated with these

- Users may download and print one copy of any publication from the public portal for the purpose of private study or research.

- You may not further distribute the material or use it for any profit-making activity or commercial gain

If the publication is distributed under the terms of Article 25fa of the Dutch Copyright Act, indicated by the "Taverne" license above, 


\section{Vascular dysfunction: at the heart of cardiovascular disease, cognitive impairment and depressive symptoms}

Thomas Teije van Sloten 
(C) Thomas van Sloten, Eindhoven, 2015

Cover picture: Bandaged Heart by Bansky - Street art located at King Street/Van Bunt Street, Brooklyn, New York, October 2013

Cover design: Wim Hilarius

Production: Ipskamp Drukkers B.V.

ISBN: 978-94-6259-637-5

Financial support by the Netherlands Heart Foundation for the publication of this thesis is greatly acknowledged. 


\title{
Vascular dysfunction: at the heart of cardiovascular disease, cognitive impairment and depressive symptoms
}

\author{
PROEFSCHRIFT \\ ter verkrijging van de graad van doctor aan de Universiteit Maastricht, \\ op gezag van de Rector Magnificus, Prof. dr. L.L.G. Soete, \\ volgens het besluit van het College van Decanen, \\ in het openbaar te verdedigen \\ op woensdag 20 mei 2015 om 14.00 uur
}

door

Thomas Teije van Sloten 


\section{Promotor}

Prof. dr. C.D.A. Stehouwer

\section{Copromotores}

Dr. R.M.A. Henry

Dr. M.T. Schram

\section{Beoordelingscommissie}

Prof. dr. H.A.J. Struijker-Boudier (voorzitter)

Prof. dr. J.K. Cruickshank (King's College, London, United Kingdom)

Prof. dr. R.J. van Oostenbrugge

Prof. dr. B.W.J.H. Penninx (VU Medisch Centrum, Amsterdam)

Dr. K.D. Reesink 


\section{Contents}

$\begin{array}{lll}\text { Chapter } 1 & \text { General introduction } & 7\end{array}$

Chapter 2 Endothelial dysfunction plays a key role in increasing cardiovascular 17 event risk in type 2 diabetes: The Hoorn Study

Chapter 3 Local stiffness of the carotid and femoral artery is associated with incident cardiovascular disease and all-cause mortality:

The Hoorn Study

Chapter 4 Carotid stiffness is associated with incident stroke: a systematic review and meta-analysis

Chapter 5 Association between arterial stiffness, cerebral small vessel disease and cognitive impairment: a systematic review and meta-analysis

Chapter 6 Cerebral small vessel disease and association with a higher incidence of depressive symptoms in a general elderly population:

The AGES-Reykjavik Study

Chapter 7 Arterial stiffness, depressive symptoms and mediation by cerebral 157 small vessel disease: The AGES-Reykjavik Study

Chapter 8 Endothelial dysfunction is associated with a greater depressive symptom score in a general elderly population: The Hoorn Study

Chapter 9 Association between arterial stiffness and skin microvascular 199 function: The SUVIMAX2 Study and The Maastricht Study

Chapter 10 General discussion

Chapter 11 Summary

$\begin{array}{ll}\text { Nederlandstalige samenvatting } & 241\end{array}$

$\begin{array}{ll}\text { List of publications } & 247\end{array}$

$\begin{array}{ll}\text { Valorization addendum } & 251\end{array}$

Dankwoord 255

$\begin{array}{ll}\text { Curriculum Vitae } & 259\end{array}$ 


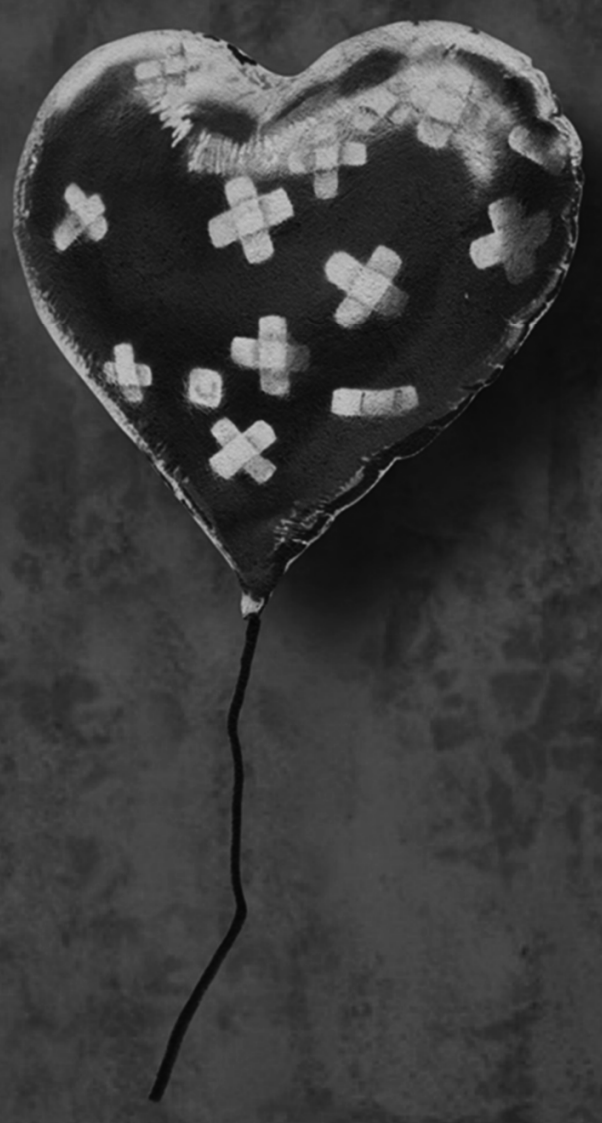

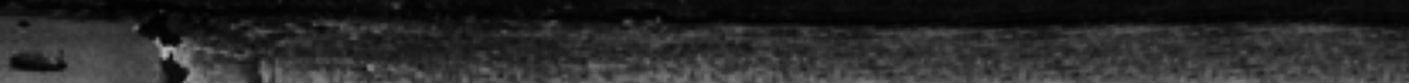
1. 1. 1. 


\section{Chapter 1}

General Introduction 

Life expectancy in the Netherlands and much of the rest of the world has dramatically increased and will continue to do so in the next decades. ${ }^{1}$ Indeed, in 2013, 2.8 million people in the Netherlands were aged 65 years or older, whereas in 2040, 4.7 million people will be aged 65 years or older ( $26.5 \%$ of the total population). ${ }^{2}$ Ageing is associated with a greatly increased risk of vascular-related diseases of the heart and brain. ${ }^{3,4}$ These include coronary heart disease, heart failure and stroke (cardiovascular diseases) and (vascular) dementia and depression. These diseases pose an enormous burden to patients, their families and health care systems and there is, thus, a growing need to understand their causes. Investigation of such causes will help to identify targets for treatment and prevention strategies. The present thesis represents an effort to investigate causes of cardiovascular disease, cognitive impairment and depressive symptoms, with a focus on the (interrelated) role of dysfunction of various elements of the vascular system (vascular dysfunction). This includes dysfunction of large arteries (due to arterial stiffness), the microcirculation (microvascular dysfunction) and endothelium (endothelial dysfunction). Emerging evidence indicates that dysfunction of these various elements is an important pathway through which ageing and other risk factors, such as type 2 diabetes and obesity, can lead to diseases of both the heart and brain. ${ }^{3-5}$

\section{Organization of the vascular system}

The vascular system branches out from a single large artery into the microvasculature, which consists of a network of arterioles, capillaries and venules. The endothelium, a single, continuous monolayer of endothelial cells, forms the inner layer of all blood vessels. The endothelium is closely related to the microvasculature, because most (98\%) of the endothelium is located in the microcirculation. ${ }^{6}$ The various elements of the vascular system have different functions. Large arteries have two main functions: to deliver blood to tissues according to their needs (conduit function) and to smooth flow pulsations imposed by the intermittent contracting heart so that blood is directed through the microcirculation in a steady stream (cushioning function). ${ }^{7}$ The microcirculation, in turn, provides a large surface area between the plasma compartment and tissue interstitium for the exchange of gases, nutrients and metabolites. In addition, the microcirculation and endothelium are involved in the regulation of many processes, including vasomotor tone, hemostatic balance, blood cell trafficking and innate and adaptive immunity. ${ }^{8,9}$

\section{Adverse effects of vascular dysfunction: underlying mechanisms}

Dysfunction of the various elements of the vascular system may lead to diseases of the heart and brain via different, but interrelated, mechanisms. Arterial stiffness leads to an impairment of the cushioning function of the arterial tree. As a consequence, pressure and flow pulsatility increase, which can manifest as increased systolic blood pressure and decreased coronary perfusion. ${ }^{10-12}$ Thereby, arterial stiffness can cause coronary heart disease, heart failure and stroke. ${ }^{10}$ In addition, the increased pulsatile load transmits distally 
and may damage the microcirculation. ${ }^{13,14}$ The brain may be especially vulnerable for this increased load, as its microcirculation is characterized by low impedance, allowing the pulsatile load to penetrate deeply into its microvascular bed. ${ }^{13,14}$ In addition, microvascular and endothelial dysfunction, defined by an impairment of any of their functions, ${ }^{15,16}$ are systemic phenomena involving vascular beds in various organs. ${ }^{17}$ Microvascular and endothelial dysfunction can lead to end-organ damage of the heart and brain via, amongst others, impairment of local tissue perfusion and blood-tissue barrier dysfunction. ${ }^{16}$ In addition, (large artery) endothelial dysfunction plays a key role in the process of atherothrombosis. ${ }^{18}$

\section{Adverse effects of vascular dysfunction: missing links}

Previous studies ${ }^{19-23}$ have shown a strong association between endothelial dysfunction, as measured by brachial artery flow-mediated dilatation ${ }^{19,20}$ or endothelium-derived circulating biomarkers, ${ }^{21-23}$ and, a higher cardiovascular event incidence. Furthermore, it has been hypothesized that individuals with type 2 diabetes are particularly prone to the detrimental effects of endothelial dysfunction on the development of cardiovascular events. $^{24,25}$ However, no studies have evaluated interaction, with regard to incident cardiovascular events, between type 2 diabetes and flow-mediated dilatation, a key functional measure of endothelium-dependent, nitric oxide-mediated dilatation. ${ }^{26}$ From a clinical point of view, detection of interaction is, however, important as this identifies key therapeutic targets: interventions aimed at such risk factors are potentially more efficacious than treatment of risk factors which do not interact.

In addition, previous meta-analyses ${ }^{27,28}$ have shown a strong association between greater aortic stiffness, as measured by carotid-femoral pulse wave velocity, and a higher cardiovascular event incidence and greater mortality risk. However, whether stiffness of other parts of the arterial tree is also associated with incident cardiovascular events is unknown. Evaluation of such associations is important, because stiffness is not uniform along the arterial tree. There are substantial differences in properties between elastic (e.g. carotid) and muscular (e.g. femoral and brachial) arteries. Stiffening of elastic and muscular arteries may lead to cardiovascular events via different mechanisms ${ }^{7,10}$ and, therefore, stiffness of these arteries may be differentially associated with cardiovascular events.

Arterial stiffness may also lead to microvascular dysfunction and damage in the brain. ${ }^{13,14}$ This can manifest itself as cerebral small vessel disease, ${ }^{29}$ and may ultimately result in cognitive impairment, including dementia. ${ }^{30-32}$ Neuroimaging markers of cerebral small vessel disease include white matter hyperintensities, cerebral microbleeds, lacunar infarcts, Virchow-Robin spaces and cerebral atrophy. ${ }^{33}$ The strength of the association between arterial stiffness and markers of cerebral small vessel disease and cognitive impairment is, however, still a matter of debate. In addition, it has been suggested ${ }^{34,35}$ that cerebral 
microvascular damage can lead to depression via disruption of deep and frontal brain structures or their connecting pathways involved in mood regulation, in particular in older individuals (vascular depression hypothesis). However, this hypothesis is controversial. ${ }^{36}$ Longitudinal data on the association between markers of cerebral small vessel disease and incident depressive symptoms are limited and inconsistent. Furthermore, the association between arterial stiffness and depressive symptoms and the potential mediating role of cerebral small vessel disease therein are yet to be determined.

Finally, it has been hypothesized that arterial stiffness leads to generalized microvascular dysfunction, i.e. dysfunction not limited to microvascular beds characterized by low impedance, such as the cerebral microcirculation. ${ }^{37}$ Such a phenomenon, if it exists, may explain the association between arterial stiffness and different diseases, including peripheral neuropathy, ${ }^{38}$ type 2 diabetes, ${ }^{39}$ and osteoporosis. ${ }^{40}$ However, evidence of an association between arterial stiffness and markers of generalized microvascular dysfunction is lacking. The skin microcirculation is a representative vascular bed to examine generalized microvascular phenomena. ${ }^{41}$ The association between arterial stiffness and skin microvascular dysfunction is, however, unknown.

\section{Cohort studies used in this thesis}

In this thesis, data are used of four cohort studies: The Hoorn Study, The Age, Gene/Environment Susceptibility (AGES)-Reykjavik Study, The Maastricht Study and The SUpplemenation en Vitamines et Mineraux AntioXydants 2 (SUVIMAX2) Study. The Hoorn Study is a population-based cohort study of glucose metabolism and cardiovascular risk among the inhabitants of the municipality of Hoorn in the Netherlands. ${ }^{42,43}$ For the present thesis, baseline and follow-up data are used of The 2000 Hoorn Study examination $(n=648) .{ }^{42}$ The AGES-Reykjavik Study is a population-based cohort study originating from The Reykjavik Study. ${ }^{44}$ From 2002 to 2006, 5,764 individuals were examined, randomly chosen from survivors of The Reykjavik Study. From 2007 to 2011 a follow-up examination was done in 3,316 of all surviving participants who agreed to participate. For the present thesis, data are used of individuals whom participated in both the baseline and follow-up examinations $(n=2,058)$. The Maastricht Study is an ongoing population-based cohort study that focuses on the etiology and pathophysiology of type 2 diabetes and the development of other chronic diseases in the general population. ${ }^{45}$ The Maastricht Study aims to include 10,000 participants from the southern part of the Netherlands. The present thesis includes data from the first 866 participants, whom completed the baseline survey between November 2010 and March 2012. Finally, The SUVIMAX Study ( $n=12,749)$ was a prevention trial designed to investigate the effect of antioxidant supplementation on cardiovascular disease and cancer, and was conducted in France between 1994 and 2002. ${ }^{46}$ In 2006 to 2007, 7,200 participants of The SUVIMAX trial participated in The SUVIMAX2 Study, an observational prospective cohort study on diet and ageing. In the present thesis, data are 


\section{2 | Chapter 1}

used of a subset of 291 individuals of The SUVIMAX2 Study, without type 2 diabetes, hypertension and cardiovascular disease, whom underwent measurements on arterial stiffness and skin microvascular function. ${ }^{47}$

\section{Outline of this thesis}

Figure 1.1 summarizes the investigated associations in this thesis (see below).

In chapter two, we investigated, in The Hoorn Study, the association between endothelial dysfunction and incident cardiovascular events, and investigated whether any such association was stronger in individuals with type 2 diabetes, impaired glucose metabolism or insulin resistance as compared to individuals with normal glucose metabolism or normal insulin sensitivity.

In chapter three, we investigated, in The Hoorn Study, the association between, on the one hand, multiple arterial stiffness indices (i.e. carotid, femoral and brachial artery stiffness, carotid-femoral pulse wave velocity, systematic arterial compliance and aortic augmentation index) and, on the other, incident cardiovascular events and all-cause mortality.

In chapter four, we conducted a systematic review and meta-analyses based on aggregate and individual participant data. We evaluated the association between carotid stiffness and incident cardiovascular events and mortality, and evaluated whether any such associations were independent of carotid-femoral pulse wave velocity. In addition, we quantified the incremental value of carotid stiffness for cardiovascular risk prediction beyond Framingham risk score factors and carotid-femoral pulse wave velocity.

In chapter five, we conducted a systematic review and an aggregate data meta-analysis of the association between, on the one hand, arterial stiffness and, on the other, markers of cerebral small vessel disease and cognitive impairment.

In chapter six, we investigated, in The AGES-Reykjavik Study, the longitudinal association between markers of cerebral small vessel disease and incident depressive symptoms.

In chapter seven, we investigated, in The AGES-Reykjavik Study, the association between arterial stiffness and depressive symptoms, and additionally investigated whether any such association was mediated by cerebral small vessel disease.

In chapter eight, we investigated, in The Hoorn Study, the association between endothelial dysfunction and depressive symptoms.

In chapter nine, we investigated the association between arterial stiffness and skin microvascular function. For this chapter, data were used of both The SUVIMAX2 Study and The Maastricht Study. 
Finally, in chapter ten we discussed the key findings of the present thesis and their clinical implications. In addition, methodological considerations and directions for future research were addressed.

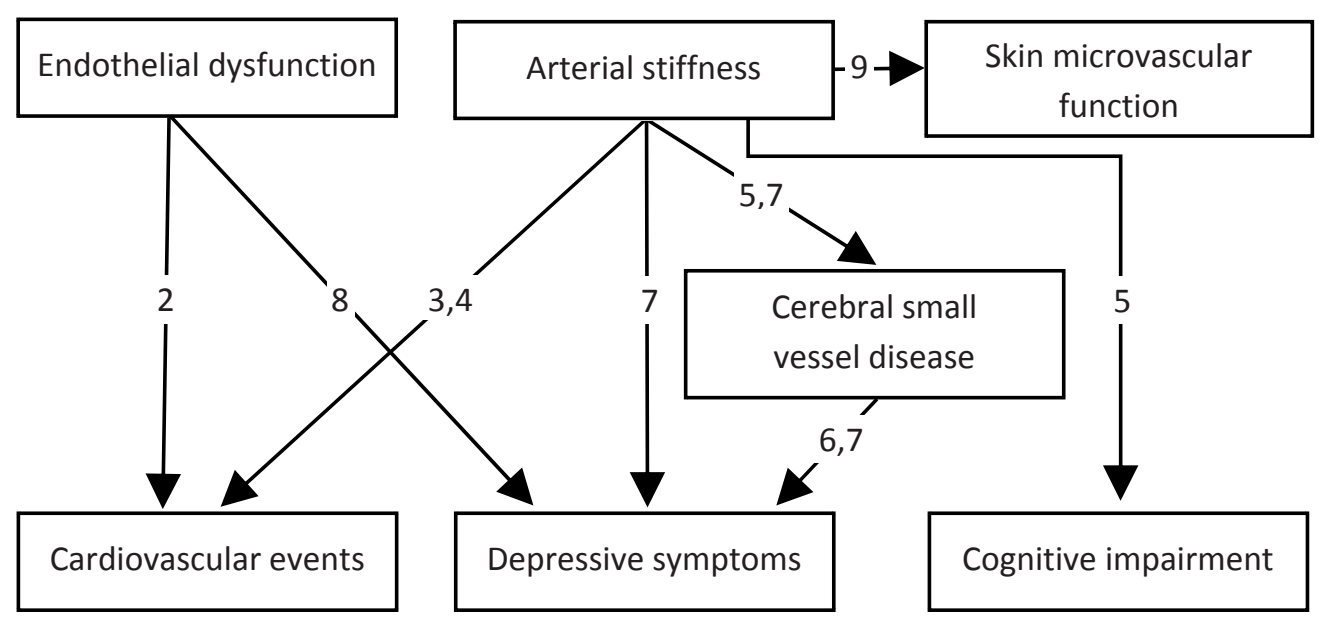

Figure 1.1. Schematic representation of the investigated associations in the present thesis. Numbers indicate corresponding chapters. 


\section{References}

1. Vaupel JW. Biodemography of human ageing. Nature. 2010;464:536-542.

2. Prognose bevolking kerncijfers, 2012-2060. Centraal Bureau voor de Statistiek. 2012.

3. Lakatta EG, Levy D. Arterial and cardiac aging: major shareholders in cardiovascular disease enterprises: Part I: aging arteries: a "set up" for vascular disease. Circulation. 2003;107:139-146.

4. Lakatta EG, Levy D. Arterial and cardiac aging: major shareholders in cardiovascular disease enterprises: Part II: the aging heart in health: links to heart disease. Circulation. 2003;107:346-354.

5. Stehouwer CD, Henry RM, Ferreira I. Arterial stiffness in diabetes and the metabolic syndrome: a pathway to cardiovascular disease. Diabetologia. 2008;51:527-539.

6. Wolinsky H. A proposal linking clearance of circulating lipoproteins to tissue metabolic activity as a basis for understanding atherogenesis. Circ Res. 1980;47:301-311.

7. Nichols WW, O'Rourke MF, Vlachopoulos C. McDonald's blood flow in arteries: Theoretical, Experimental and Clinical Principles, 6th edition. London: Hodder Arnold; 2011.

8. Aird WC. Phenotypic heterogeneity of the endothelium: II. Representative vascular beds. Circ Res. 2007;100:174-190.

9. Aird WC. Phenotypic heterogeneity of the endothelium: I. Structure, function, and mechanisms. Circ Res. 2007;100:158-173.

10. Laurent S, Cockcroft J, Van Bortel L, et al. Expert consensus document on arterial stiffness: methodological issues and clinical applications. Eur Heart J. 2006;27:2588-2605.

11. Kaess BM, Rong J, Larson MG, et al. Aortic stiffness, blood pressure progression, and incident hypertension. JAMA. 2012;308:875-881.

12. Weisbrod RM, Shiang T, Al Sayah L, et al. Arterial stiffening precedes systolic hypertension in dietinduced obesity. Hypertension. 2013;62:1105-1110.

13. Mitchell GF. Effects of central arterial aging on the structure and function of the peripheral vasculature: implications for end-organ damage. J Appl Physiol (1985). 2008;105:1652-1660.

14. O'Rourke MF, Safar ME. Relationship between aortic stiffening and microvascular disease in brain and kidney: cause and logic of therapy. Hypertension. 2005;46:200-204.

15. Schalkwijk CG, Stehouwer CD. Vascular complications in diabetes mellitus: the role of endothelial dysfunction. Clin Sci (Lond). 2005;109:143-159.

16. Muris DM, Houben AJ, Schram MT, Stehouwer CD. Microvascular dysfunction: an emerging pathway in the pathogenesis of obesity-related insulin resistance. Rev Endocr Metab Disord. 2013;14:29-38.

17. Vita JA. Endothelial function. Circulation. 2011;124:e906-912.

18. Hansson GK. Inflammation, atherosclerosis, and coronary artery disease. N Eng/ J Med. 2005;352:16851695.

19. Yeboah J, Crouse JR, Hsu FC, Burke GL, Herrington DM. Brachial flow-mediated dilation predicts incident cardiovascular events in older adults: the Cardiovascular Health Study. Circulation. 2007;115:23902397.

20. Yeboah J, Folsom AR, Burke GL, et al. Predictive value of brachial flow-mediated dilation for incident cardiovascular events in a population-based study: the multi-ethnic study of atherosclerosis. Circulation. 2009;120:502-509.

21. Blankenberg S, Rupprecht HJ, Bickel C, et al. Circulating cell adhesion molecules and death in patients with coronary artery disease. Circulation. 2001;104:1336-1342.

22. Empana JP, Sykes DH, Luc G, et al. Contributions of depressive mood and circulating inflammatory markers to coronary heart disease in healthy European men: the Prospective Epidemiological Study of Myocardial Infarction (PRIME). Circulation. 2005;111:2299-2305.

23. Jager A, van Hinsbergh VW, Kostense PJ, et al. von Willebrand factor, C-reactive protein, and 5-year mortality in diabetic and nondiabetic subjects: the Hoorn Study. Arterioscler Thromb Vasc Biol. 1999;19:3071-3078.

24. de Jager J, Dekker JM, Kooy A, et al. Endothelial dysfunction and low-grade inflammation explain much of the excess cardiovascular mortality in individuals with type 2 diabetes: the Hoorn Study. Arterioscler Thromb Vasc Biol. 2006;26:1086-1093.

25. Frankel DS, Meigs JB, Massaro JM, et al. Von Willebrand factor, type 2 diabetes mellitus, and risk of cardiovascular disease: the framingham offspring study. Circulation. 2008;118:2533-2539.

26. Flammer AJ, Anderson T, Celermajer DS, et al. The assessment of endothelial function: from research into clinical practice. Circulation. 2012;126:753-767. 
27. Ben-Shlomo Y, Spears M, Boustred C, et al. Aortic pulse wave velocity improves cardiovascular event prediction: an individual participant meta-analysis of prospective observational data from 17,635 subjects. J Am Coll Cardiol. 2014; 25:636-646

28. Vlachopoulos C, Aznaouridis K, Stefanadis C. Prediction of cardiovascular events and all-cause mortality with arterial stiffness: a systematic review and meta-analysis. J Am Coll Cardiol. 2010;55:1318-1327.

29. Wardlaw JM, Smith C, Dichgans M. Mechanisms of sporadic cerebral small vessel disease: insights from neuroimaging. Lancet Neurol. 2013;12:483-497.

30. Mitchell GF, van Buchem MA, Sigurdsson S, et al. Arterial stiffness, pressure and flow pulsatility and brain structure and function: the Age, Gene/Environment Susceptibility--Reykjavik study. Brain. 2011;134:3398-3407.

31. Tsao CW, Seshadri S, Beiser AS, et al. Relations of arterial stiffness and endothelial function to brain aging in the community. Neurology. 2013;81:984-991.

32. Hanon $\mathrm{O}$, Haulon $\mathrm{S}$, Lenoir $\mathrm{H}$, et al. Relationship between arterial stiffness and cognitive function in elderly subjects with complaints of memory loss. Stroke. 2005;36:2193-2197.

33. Wardlaw JM, Smith EE, Biessels GJ, et al. Neuroimaging standards for research into small vessel disease and its contribution to ageing and neurodegeneration. Lancet Neurol. 2013;12:822-838.

34. Alexopoulos GS, Meyers BS, Young RC, Campbell S, Silbersweig D, Charlson M. 'Vascular depression' hypothesis. Arch Gen Psychiatry. 1997;54:915-922.

35. Krishnan KR, Hays JC, Blazer DG. MRI-defined vascular depression. Am J Psychiatry. 1997;154:497-501.

36. Newson RS, Hek K, Luijendijk HJ, Hofman A, Witteman JC, Tiemeier H. Atherosclerosis and incident depression in late life. Arch Gen Psychiatry. 2010;67:1144-1151.

37. Mitchell GF, Vita JA, Larson MG, et al. Cross-sectional relations of peripheral microvascular function, cardiovascular disease risk factors, and aortic stiffness: the Framingham Heart Study. Circulation. 2005;112:3722-3728.

38. Kim ES, Moon SD, Kim HS, et al. Diabetic peripheral neuropathy is associated with increased arterial stiffness without changes in carotid intima-media thickness in type 2 diabetes. Diabetes Care. 2011;34:1403-1405.

39. Yasuno S, Ueshima K, Oba K, et al. Is pulse pressure a predictor of new-onset diabetes in high-risk hypertensive patients?: a subanalysis of the Candesartan Antihypertensive Survival Evaluation in Japan (CASE-J) trial. Diabetes Care. 2010;33:1122-1127.

40. Giallauria F, Ling SM, Schreiber C, et al. Arterial stiffness and bone demineralization: the Baltimore longitudinal study of aging. Am J Hypertens. 2011;24:970-975.

41. Holowatz LA, Thompson-Torgerson CS, Kenney WL. The human cutaneous circulation as a model of generalized microvascular function. J Appl Physiol (1985). 2008;105:370-372.

42. Henry RM, Kostense PJ, Spijkerman AM, et al. Arterial stiffness increases with deteriorating glucose tolerance status: the Hoorn Study. Circulation. 2003;107:2089-2095.

43. Mooy JM, Grootenhuis PA, de Vries H, et al. Prevalence and determinants of glucose intolerance in a Dutch caucasian population. The Hoorn Study. Diabetes Care. 1995;18:1270-1273.

44. Harris TB, Launer LJ, Eiriksdottir G, et al. Age, Gene/Environment Susceptibility-Reykjavik Study: multidisciplinary applied phenomics. Am J Epidemiol. 2007;165:1076-1087.

45. Schram MT, Sep SJ, van der Kallen CJ, et al. The Maastricht Study: an extensive phenotyping study on determinants of type 2 diabetes, its complications and its comorbidities. Eur J Epidemiol. 2014;29:439451.

46. Hercberg S, Galan P, Preziosi P, et al. The SU.VI.MAX Study: a randomized, placebo-controlled trial of the health effects of antioxidant vitamins and minerals. Arch Intern Med. 2004;164:2335-2342.

47. Karatzi K, Protogerou A, Kesse-Guyot E, et al. Associations between dietary patterns and skin microcirculation in healthy subjects. Arterioscler Thromb Vasc Biol. 2014;34:463-469. 


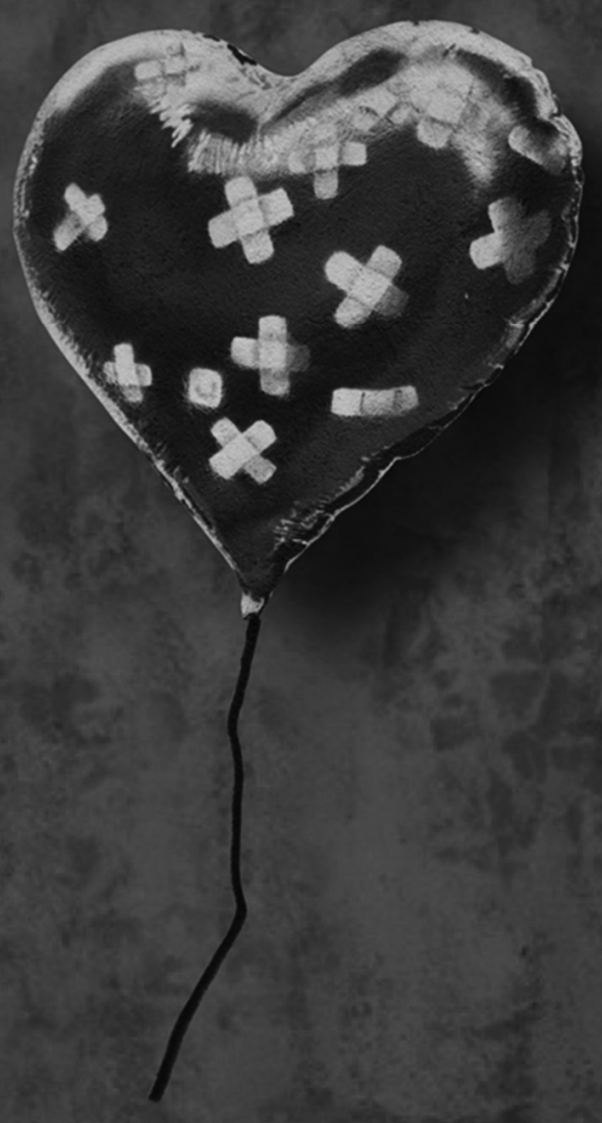

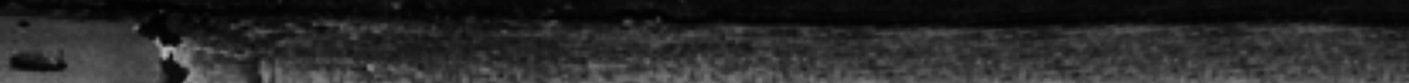
1. 1. 1. 


\section{Chapter 2}

\section{Endothelial dysfunction plays a key role}

in increasing cardiovascular event risk in type 2 diabetes: The Hoorn Study

TT van Sloten, RMA Henry, JM Dekker, G Nijpels, T Unger, MT Schram, CDA Stehouwer 
18 | Chapter 2

\begin{abstract}

\section{Background}

In the pathogenesis of cardiovascular events, interaction between risk factors has seldom been identified. However, endothelial dysfunction on the one hand and type 2 diabetes, impaired glucose metabolism and insulin resistance on the other may act synergistically (i.e. interact) in the development of cardiovascular disease. We therefore investigated the interaction between endothelial dysfunction and type 2 diabetes, impaired glucose metabolism and insulin resistance with regard to risk of cardiovascular events.
\end{abstract}

\title{
Methods
}

In a prospective population-based cohort ( $n=445$; mean age 69 years; $55 \%$ women; $23 \%$ type 2 diabetes, and $28 \%$ impaired glucose metabolism (by design)), endothelial dysfunction (brachial artery flow-mediated dilatation), glucose tolerance (oral glucose tolerance test) and insulin sensitivity (homeostasis model assessment for insulin resistance, HOMA2-IR) were determined.

\section{Results}

After a median follow-up of 7.6 years, 106 participants had had a cardiovascular event. After adjustments, one standard deviation less flow-mediated dilatation was associated with cardiovascular events in type 2 diabetes (hazard ratio 1.69 [95\% confidence interval 1.14 to 2.52]) and impaired glucose metabolism (1.50 [0.95 to 2.37]), and among those in the highest HOMA2-IR tertile (1.92 [1.42 to 2.60]), but not in normal glucose metabolism (0.85 [0.63 to 1.16]) or among those in the lower two HOMA2-IR tertile tertiles combined (0.85 [0.65 to 1.12]). Interaction between flow-mediated dilatation and type 2 diabetes, impaired glucose metabolism or insulin resistance was present on an additive (relative excess risk due to interaction $>0$ ) and a multiplicative scale (P-interaction $<.05)$.

\section{Conclusion}

Endothelial dysfunction and type 2 diabetes, impaired glucose metabolism or insulin resistance synergistically increase cardiovascular event risk. This identifies endothelial dysfunction as a key therapeutic target in these individuals. 


\section{Introduction}

In the pathogenesis of cardiovascular (CV) events, true interaction (i.e. synergy) between risk factors appears rare, i.e. most studies find that risk factors act, and thus increase CV event risk, independently of each other. ${ }^{1-3}$ However, it has been hypothesized that individuals with type 2 diabetes mellitus (DM2) are particularly prone to the detrimental effects of endothelial dysfunction, ${ }^{4-6}$ a key mechanism in the pathogenesis of atherothrombosis, and that this may explain the increased CV events risk in DM2. If true, this implies that DM2 and endothelial dysfunction interact with regard to the pathogenesis of CV events. That is, DM2 and endothelial dysfunction may act more strongly in the presence of the other variable than in its absence. From a clinical point of view, detection of interaction between risk factors is important as this identifies key therapeutic targets: interventions aimed at such risk factors are potentially more efficacious than treatment of risk factors which do not interact.

The mechanism that may underlie this phenomenon is a bidirectional association between endothelial dysfunction and DM2, in which endothelial dysfunction may act as both cause ${ }^{6,7}$ and consequence ${ }^{6,8}$ of DM2. On the one hand, DM2 leads to endothelial dysfunction via, amongst others, formation of advanced glycation end products (AGEs), intra-endothelial accumulation of glucose and increased oxidative stress. ${ }^{6,8}$ On the other hand, endothelial dysfunction causes or aggravates DM2 by impairing the timely access of glucose and insulin to their target tissues. ${ }^{7}$ Consequently, a vicious circle may exist between endothelial dysfunction and DM2. In addition, DM2 may amplify the detrimental effects of endothelial dysfunction on atherothrombosis via multiple pathways, including (mitochondrial) overproduction of reactive oxygen species, low-grade inflammation and increased procoagulant activity and platelet aggregation. ${ }^{9}$ Similar mechanisms may be operative in individuals with impaired glucose metabolism (IGM; i.e. impaired fasting glucose and/or impaired glucose tolerance) or with insulin resistance but with normal glucose tolerance, in whom an increased risk of CV events is also apparent. ${ }^{10,11}$

If the above hypothesis is correct, then the co-occurrence of endothelial dysfunction and DM2 will increase CV event risk more than expected on the basis of the presence of these processes alone. This phenomenon is called causal interaction or interaction on an additive scale, ${ }^{12,13}$ and can be formally tested in observational data through the calculation of the relative excess risk due to interaction (RERI). ${ }^{12}$

To date, two previous studies, an earlier report of The Hoorn Study ${ }^{4}$ and The Framingham Offspring Study, ${ }^{5}$ have evaluated the joint effects of endothelial dysfunction, as determined by plasma biomarkers, and DM2 on incident CV events. In agreement with the above hypothesis, these studies showed that endothelial dysfunction was most strongly 
associated with incident CV events in individuals with DM2 as compared to those without DM2. However, these studies did not evaluate causal interaction (i.e. interaction on an additive scale). In addition, these studies did not measure flow-mediated dilatation (FMD), a key functional measure of endothelium-dependent, nitric oxide (NO)-mediated dilatation. $^{14}$

In view of the above, we investigated, in a general elderly population, the association between endothelial dysfunction, as determined by FMD, and incident CV events, and formally tested, for the first time, whether any such association was stronger in individuals with DM2, IGM and/or insulin resistance as compared to individuals with normal glucose metabolism (NGM) or normal insulin sensitivity (i.e. the presence of causal interaction).

\section{Methods}

\section{Study design}

For the present study, we used data from The 2000 Hoorn Study follow-up examination. The Hoorn Study is a population-based cohort study of glucose metabolism and CV risk among inhabitants of the municipality of Hoorn in the Netherlands. Details of the study have been described elsewhere. ${ }^{4,14,15}$ The Hoorn Study was approved by the Ethical Review Committee of the VU University Medical Centre. Informed consent was obtained from all participants.

\section{Brachial artery FMD}

A detailed description of the measurement of FMD and nitroglycerin-mediated dilatation (NMD) is provided in the supplemental material.

\section{Determination of glucose metabolism and insulin resistance status}

All participants, except those with previously diagnosed diabetes, underwent a standard 75-g oral glucose tolerance test and were classified as having either NGM, IGM or DM2 according to the 1999 World Health Organization criteria. ${ }^{16}$ Insulin resistance was estimated using the homeostasis model assessment for insulin resistance (HOMA2-IR) calculator (www.dtu.ox.ac.uk).

\section{Other measurements}

CV risk factors and prior cardiovascular disease (CVD) were assessed as described previously. ${ }^{4,14,15}$ 


\section{Follow-up}

Follow-up was complete until January 1, 2009. Information on CV morbidity and mortality was extracted from medical records of general practitioners and the local hospital, and classified according to the International Classification of Disease ( $9^{\text {th }}$ edition; ICD-9). We defined incident CV events (non-fatal and fatal combined) as ICD-9 codes: 410-414 (coronary heart disease, CHD), 427-428 (heart failure), 431-438 (cerebrovascular disease), 440-443 (arterial disease), 798 (sudden death), and ICD-9 clinical modification code 36 (coronary arterial procedures). Data on the participants' vital status were collected from the municipal population register. We determined for each participant whether or not death had occurred during follow-up, and if so, the date when death occurred.

\section{Statistical analysis}

All analyses were performed with $R$ statistical software (version 2.15.2). For insulin resistance status, individuals were classified by the highest HOMA2-IR tertile vs. the lower tertiles combined. The lower tertiles were combined, because results for these tertiles did not materially differ from each other in all analyses (i.e. there was a non-linear interaction between FMD and HOMA2-IR levels, see below). In all analyses, FMD was adjusted for baseline diameter, flow increase after cuff release and NMD. Results were adjusted for NMD, a measure of endothelial-independent vasodilatation, because less FMD may be due to impaired endothelial and/or endothelial-independent (smooth muscle cell) function. ${ }^{14}$ Cox proportional hazard models were used to estimate the association between FMD and incident CV events. First, the association between FMD and CV events was evaluated in the total study population. This association was adjusted for age and sex (cohort stratifying variables, model 1); and additionally for prior CVD, body mass index (BMI), total/HDL cholesterol, triglycerides, hypertension, estimated glomerular filtration rate (eGFR), physical activity, smoking habits, the use of anti-hypertensive and lipid-lowering medication (potential confounders, model 2); glucose metabolism status (i.e. DM2, IGM and NGM) (model 3); and insulin resistance status (i.e. highest HOMA2-IR tertile and lower two tertiles combined) (model 4). Second, analyses were repeated after stratification for glucose metabolism or insulin resistance status. Third, we investigated the presence of potentially causal interactions on an additive scale (i.e. when risk factors act synergistically in causing disease ${ }^{13}$ ). In Cox regression analysis, however, statistical interaction is exponential and, therefore, multiplicative. To nevertheless evaluate the presence of additive interaction, we calculated the RERI. ${ }^{12}$ RERI represents the risk that is in excess of what would be expected if there had been no additive interaction. $A$ RERI $>0$ indicates positive additive interaction. ${ }^{12}$ In these analyses, adjustments were made for the same sets of potential confounders as described for the Cox regression models. Confidence intervals of the RERI were estimated by using a bootstrap method with 10,000 samples. ${ }^{12}$ Finally, we also calculated the presence of any multiplicative interaction by adding, to our Cox regression models, product terms between FMD and DM2, IGM and insulin resistance. 


\section{Results}

\section{Study population}

Of the 648 participants, qualitatively sufficient ultrasound examinations were obtained in 492 individuals. Data were missing for logistical reasons $(n=49)$ and poor definition of the arterial wall due to obesity $(n=107)$. In addition, participants were excluded when data on glucose metabolism status ( $n=8$ ) or CV event follow-up were missing ( $n=39$; of whom 6 had moved out of town and could not be contacted; and 33 did not give permission to access their medical files or to contact their general practitioner). Thus, 445 participants were eligible for the present analyses. Individuals without follow-up data did not differ from the study population (data not shown).

\section{Clinical characteristics}

Table 2.1 shows the baseline characteristics of the study population according to CV event status. The median duration of follow-up was 7.6 years (range 0.2 to 8.9). A total of 106 participants (42 NGM, 33 IGM, 31 DM2) had a CV event, 12 (11.3\%) of which were fatal. A total of 48 participants had a CHD event (16 NGM, 19 IGM, 13 DM2), 35 had a cerebrovascular event (16 NGM, 10 IGM, 9 DM2) and 23 had a CV event other than a CHD or cerebrovascular event (e.g. peripheral arterial disease or heart failure). The incidence rate of CV events was 3.1\% per year. Individuals with an incident CV event were older and more often men. In addition, these individuals suffered more often from DM2, had greater insulin resistance as determined by HOMA2-IR and a less favorable CV risk profile (Table 2.1). In addition, individuals with a CV event had less FMD, a smaller baseline diameter and a lower flow increase after cuff release (Table 2.1).

\section{Association between FMD and incident CV events}

Multivariable cox regression analysis showed that FMD was not significantly associated with incident CV events in the overall population (Table 2.2, models 1 to 4). However, when the analyses were repeated stratified according to glucose metabolism or insulin resistance status, the results showed that, after adjustment for potential confounders, less FMD was associated with CV events in individuals with DM2 and IGM, but not in individuals with NGM (Table 2.2, model 2; also illustrated in Figure 2.1, panel A). Similarly, less FMD was associated with CV events among those in the highest HOMA2-IR tertile, but not among those in the lower two tertiles combined (Table 2.2, model 2; also illustrated in Figure 2.1, panel B). These results did not materially change when we additionally adjusted glucose metabolism and insulin resistance status for each other (models 3 ). 
Table 2.1. Clinical characteristics of the study population at baseline according to incident cardiovascular event status

\begin{tabular}{|c|c|c|}
\hline & $\begin{array}{c}\text { Participants without a } \\
\text { cardiovascular event } \\
n=339(76.2 \%)\end{array}$ & $\begin{array}{c}\text { Participants with } \\
\text { a cardiovascular event } \\
n=106(23.8 \%)\end{array}$ \\
\hline \multicolumn{3}{|l|}{ General characteristics } \\
\hline Age (years) & $68.5 \pm 6.2$ & $71.4 \pm 6.1$ \\
\hline Women & 55.5 & 35.8 \\
\hline \multicolumn{3}{|l|}{ Smoking habits } \\
\hline Current smoker & 10.9 & 21.7 \\
\hline Former smoker & 44.0 & 49.1 \\
\hline Non-smoker & 45.1 & 29.2 \\
\hline Physical activity (MET hours / week) & $82(49-128)$ & $77(48-125)$ \\
\hline Prior cardiovascular disease & 47.5 & 63.2 \\
\hline \multicolumn{3}{|l|}{ Glucose metabolism status } \\
\hline Type 2 diabetes & 20.9 & 29.2 \\
\hline Impaired glucose metabolism & 26.8 & 31.1 \\
\hline Normal glucose metabolism & 52.3 & 39.7 \\
\hline \multicolumn{3}{|l|}{ Insulin resistance status } \\
\hline HOMA2-IR & $1.00(0.80-1.50)$ & $1.20(0.80-1.63)$ \\
\hline HOMA2-IR tertile 3 & 31.0 & 37.3 \\
\hline HOMA2-IR tertile 2 & 38.8 & 31.4 \\
\hline HOMA2-IR tertile 1 & 30.2 & 31.3 \\
\hline Body mass index $\left(\mathrm{kg} / \mathrm{m}^{2}\right)$ & $26.7 \pm 3.3$ & $27.2 \pm 3.4$ \\
\hline Systolic blood pressure (mmHg) & $141 \pm 20$ & $148 \pm 21$ \\
\hline Diastolic blood pressure (mmHg) & $82 \pm 11$ & $83 \pm 11$ \\
\hline Hypertension & 62.8 & 79.2 \\
\hline $\mathrm{HbA1c}(\mathrm{mmol} / \mathrm{mol})$ & $41 \pm 7$ & $44 \pm 9$ \\
\hline $\mathrm{HbA1c}(\%)$ & $5.9 \pm 0.7$ & $6.2 \pm 0.8$ \\
\hline Total cholesterol (mmol/L) & $5.8 \pm 1.0$ & $5.8 \pm 1.1$ \\
\hline LDL cholesterol (mmol/L) & $3.7 \pm 0.9$ & $3.8 \pm 0.9$ \\
\hline HDL cholesterol (mmol/L) & $1.5 \pm 0.4$ & $1.3 \pm 0.4$ \\
\hline Triglycerides (mmol/L) & $1.2(0.9-1.7)$ & $1.4(1.1-1.8)$ \\
\hline Estimated glomerular filtration rate $\left(\mathrm{ml} / \mathrm{min} / 1.73 \mathrm{~m}^{2}\right)$ & $62.7 \pm 10.0$ & $60.2 \pm 11.0$ \\
\hline (Micro)albuminuria (albumin/creatinine ratio $>2 \mathrm{mg} / \mathrm{mmol}$ ) & 10.9 & 20.0 \\
\hline \multicolumn{3}{|l|}{ Medication use } \\
\hline Lipid-lowering medication & 12.4 & 19.8 \\
\hline Anti-hypertensive medication & 28.9 & 44.3 \\
\hline \multicolumn{3}{|l|}{ Flow-mediated dilatation } \\
\hline Flow-mediated dilatation (mm) & $0.19 \pm 0.15$ & $0.14 \pm 0.20$ \\
\hline Flow-mediated percentage change in diameter (\%) & $4.3 \pm 3.7$ & $3.1 \pm 4.0$ \\
\hline Baseline diameter $(\mathrm{mm})$ & $4.59 \pm 0.75$ & $4.83 \pm 0.69$ \\
\hline Flow increase after cuff release (\%) & $91 \pm 45$ & $79 \pm 40$ \\
\hline Flow increase after cuff release $(\mathrm{cm} / \mathrm{s})$ & $11.3 \pm 6.0$ & $9.6 \pm 4.8$ \\
\hline
\end{tabular}

Values are \%, mean \pm SD or median (interquartile range).

HOMA2-IR = homeostasis model assessment for insulin resistance; HbA1c = glycated hemoglobin; LDL = low density lipoprotein; $\mathrm{HDL}$ = high density lipoprotein; $\mathrm{MET}$ = metabolic equivalent of task. 

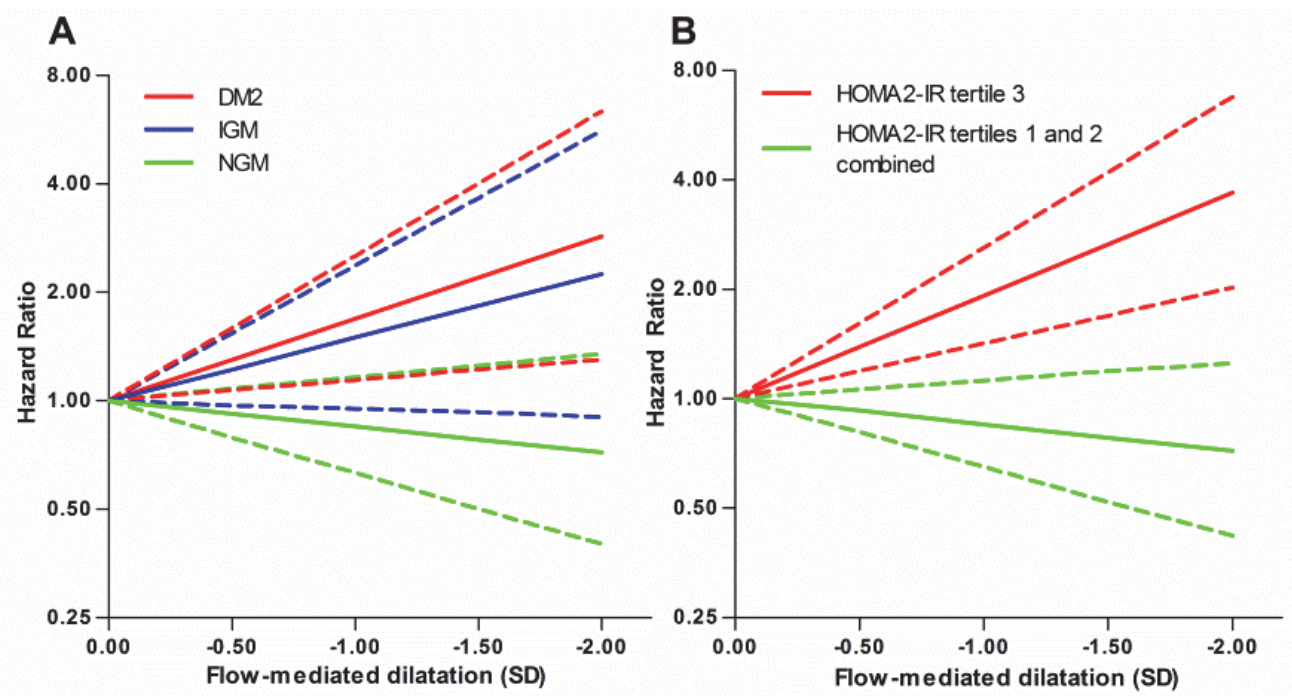

Figure 2.1. Associations of flow-mediated dilatation with incident cardiovascular events stratified according to glucose metabolism status (type 2 diabetes mellitus (DM2), impaired glucose metabolism (IGM) and normal glucose metabolism (NGM)) (panel A) and insulin resistance status (HOMA2-IR tertiles) (panel B). All results are adjusted for potential confounders (see text). Solid lines indicate estimated effect; dashed lines indicate corresponding $95 \%$ confidence intervals.

\section{Interaction analyses}

When the analyses were repeated to test for additive interaction between FMD and glucose metabolism status, the results showed that, after adjustment for potential confounders, the RERI, per one standard deviation (SD) less FMD, was 0.64 ( $95 \%$ confidence interval -0.35 to 1.32) for DM2 vs. NGM and 0.68 (-0.07 to 1.93) for IGM vs. NGM, respectively (Table 2.2, model 2). This means that the HRs for incident CV events in DM2 and IGM were, per one SD less FMD, 0.64 and 0.68 higher, respectively, than if there had been no interaction between FMD and DM2 or IGM. Similarly, the RERI, per one SD less FMD, was 0.73 (0.30 to 1.34) for the highest HOMA2-IR tertile vs. the lower two tertiles combined (Table 2.2, model 2).

When the analyses were repeated to test for multiplicative interaction, the results showed that, after adjustment for potential confounders, the HRs, per one SD less FMD, for the product terms between FMD and DM2 or IGM vs. NGM were 1.96 (1.21 to 3.17) and 1.76 (1.03 to 2.98), respectively (Table 2.2, model 2). This means that the HRs for incident CV events in DM2 and IGM were, per one SD less FMD, 1.96 and 1.76 times higher, respectively, than if there had been no multiplicative interaction between FMD and DM2 or IGM. Similarly, the HR, per one SD less FMD, for the product terms between FMD and the highest HOMA2-IR tertile vs. the lower two tertiles combined was 2.25 (1.53 to 3.32) (Table 2.2, model 2 ). These results did not materially change when we additionally adjusted glucose metabolism and insulin resistance status for each other (models 3 ). 


\section{Additional analyses}

There was no additive or multiplicative interaction between FMD and $\mathrm{HbA} 1 \mathrm{c}$, fasting or postload glucose in the association with CV events (data not shown). In addition, the results of the interaction analyses between FMD and DM2, IGM and insulin resistance did not materially change when we additionally adjusted for $\mathrm{HbA1c}$, fasting or postload glucose (data not shown).

All analyses were then repeated with incident CV events as the outcome, but with incident heart failure $(n=9)$ excluded from the definition of CV events. The results of these analyses were qualitatively similar to the analyses with total incident CV events as the outcome (see supplemental material, Table S2.1)

Next, all analyses were repeated with all-cause mortality as outcome. These analyses showed that FMD was not associated with all-cause mortality. In addition, there was no additive or multiplicative interaction between FMD and DM2, IGM or insulin resistance in the association with all-cause mortality (see supplemental material, Table S2.2).

Endothelial function is closely linked to low-grade inflammation. Any association of endothelial dysfunction with incident CV events and/or mortality may, thus, be dependent on low-grade inflammation. We therefore determined circulating biomarkers of low-grade inflammation (high-sensitivity C-reactive protein, serum amyloid A, interleukin-6, interleukin-8, tumor necrosis factor- $\alpha$ and soluble intracellular adhesion molecule-1), and constructed a summarizing low-grade inflammation Z-score as described previously. ${ }^{17}$ When we additionally adjusted the results for this Z-score, results did not materially change (data not shown).

Estimation of insulin resistance based on HOMA2-IR may be less accurate in individuals treated with insulin. ${ }^{18}$ When we excluded these individuals $(n=10)$ from the analyses, however, results did not materially change (data not shown).

The associations between FMD and incident CV events may differ according to the presence of prior CVD. However, no interaction was observed on an additive (RERI -0.17 [-1.09 to 0.74]) or a multiplicative scale (HR for the product term between FMD and prior CVD vs. no prior CVD was 0.82 [0.50 to 1.33$]$ ).

Finally, the associations between FMD and incident CV events may differ according to DM2 duration. However, no interaction was observed on an additive (RERI -0.03 (-0.25 to 0.32)) or a multiplicative scale (HR for the product term between FMD and DM2 duration was 0.96 (0.90 to 1.04). 


\section{Discussion}

This population-based study is the first that formally tests the joint effect, on incident CV events, of FMD on the one hand and DM2, IGM and insulin resistance on the other. We observed that FMD was most strongly associated with CV events in individuals with DM2 or insulin resistance, less strongly in IGM and not associated with incident CV events in individuals with NGM or normal insulin sensitivity. Importantly, the increased CV events risk of the joint effect of endothelial dysfunction and DM2, IGM or insulin resistance was greater than what would have been expected had the effect of FMD on the one hand and glucose metabolism or insulin resistance status on the other acted independently of each other (as indicated by a RERI $>0$ ), demonstrating the presence of interaction or synergy between endothelial dysfunction and impairment of glucose metabolism with respect to CV event risk.

The present study defined endothelial dysfunction as impaired endothelium-dependent FMD, which is a key functional estimate of endothelial function. ${ }^{14}$ The study thereby extends previous studies ${ }^{4,5}$ on the joint effects of endothelial dysfunction and DM2, IGM or insulin resistance on incident $\mathrm{CV}$ events, which used plasma biomarkers to define endothelial dysfunction and showed multiplicative interaction between endothelial dysfunction and DM2. No information was, however, provided on an additive interaction scale (i.e. potentially causal interaction ${ }^{12,13}$ ). The present study therefore provides additional and strong evidence in favor of a causal interaction between endothelial dysfunction on the one hand and DM2, IGM and insulin resistance on the other in the pathogenesis of CV events.

Causal interaction between two factors means mutual dependence in causing disease, i.e. such factors are component causes in the same causal model. Rothman ${ }^{13}$ and others ${ }^{12}$ have argued that potentially causal interaction needs to be evaluated as departure from additivity rather than departure from multiplicativity. In the present study, interaction was present on an additive (as indicated by RERI) as well as on a multiplicative scale (as indicated by interaction terms in regression analyses). Not all interaction tests were, however, statistically significant. This may be due to the fact that, in general, these tests are limited by relatively low statistical power.

The mechanism that may underlie this interaction is the presence of a vicious circle between endothelial dysfunction and DM2, IGM and insulin resistance, ${ }^{6}$ with, on the one hand, DM2, IGM and insulin resistance causing endothelial dysfunction and, on the other, endothelial dysfunction causing insulin resistance, IGM and DM2.7,8 There is abundant evidence that DM2, IGM and insulin resistance cause endothelial dysfunction, ${ }^{9}$ but evidence for the reverse process is relatively recent. ${ }^{7}$ However, insulin normally can redirect blood flow in skeletal muscle from non-nutritive capillaries to nutritive capillaries and, thereby, increase 
insulin-mediated glucose uptake. ${ }^{19}$ These processes are impaired by endothelial dysfunction. ${ }^{7}$ In addition, endothelial dysfunction may also cause apoptosis of beta-cells in the pancreas, ${ }^{20}$ which decreases insulin secretory capacity and, therefore, may, further impair glucose metabolism. In addition, DM2 may amplify the detrimental effects of endothelial dysfunction on atherothrombosis. ${ }^{9}$ Hence, the co-occurrence of these processes may accelerate atherothrombosis and, thus, increase $\mathrm{CV}$ event risk more than expected from the presence of these processes alone.

In the present study, glucose metabolism and insulin resistance states interacted with endothelial dysfunction independently of each other. This suggests that mechanisms associated with DM2 and IGM other than insulin resistance may play a role in the interaction with endothelial dysfunction, such as AGEs, oxidative stress and diabetic dyslipidemia. ${ }^{9}$

Somewhat surprisingly, estimates of hyperglycemia (i.e. HbA1c, fasting or postload glucose) did not interact with endothelial dysfunction in the association with $\mathrm{CV}$ events. This finding may have several explanations. First, only a single measurement of (baseline) variables was available in the present study. Baseline glucose levels may not accurately reflect exposure during follow-up. Second, glucose levels may not accurately reflect the mechanisms by which hyperglycemia leads to endothelial dysfunction and/or CV events (i.e. AGEs, and/or oxidative stress). ${ }^{9}$ Third, it has been suggested that glucose variability ${ }^{21}$ and episodes of hypoglycemia ${ }^{22}$ may be more strongly associated with endothelial dysfunction than mean blood glucose levels (i.e. HbA1c). Nevertheless, we cannot exclude the play of chance.

From a clinical point of view, the synergistic association between endothelial dysfunction and DM2 is important as endothelial dysfunction may act at least partially as the underlying phenomenon which might explain the two to three times higher CV events risk seen in DM2. This suggests that endothelial dysfunction is a key therapeutic target for lowering of CVD risk in DM2. In addition, the fact that an interaction was already present in individuals with IGM and insulin resistance identifies endothelial dysfunction as an early therapeutic target even before DM2 is present. This is in accordance with the hypothesis that insulin resistance, IGM and DM2 are manifestations of a continuous disease process to increase the risk of CV events ("ticking clock hypothesis"). ${ }^{10,11}$

Our study had some limitations. First, it is likely that survival bias affected our results, i.e. it is probable that individuals who died before the start of the present study were those with the strongest association between endothelial dysfunction and/or DM2 and incident CV events. Such bias would, however, have led to an underestimation of the reported associations and may explain why we did not find an association between FMD and incident CV events in individuals with NGM. Second, the CV event rate in the present study population at high CV risk was in accordance with previous studies. ${ }^{10}$ However, a relatively 
low number (11.3\%) of participants died of a CV event, which may reflect a survival effect and/or may be due to the high quality of CVD management in the Netherlands. This low fatal CV event rate may explain the lack of an interaction between endothelial dysfunction and glucose metabolism or insulin resistance in the association with all-cause mortality. Third, the present study had insufficient power to evaluate interaction, with regard to specific CV events, between FMD on the one hand and glucose metabolism status en insulin resistance on the other. Finally, only a single (baseline) measurement of FMD was available. Baseline FMD may not accurately reflect exposure during follow-up, and this may have led to an underestimation of the reported associations.

In conclusion, the present study shows that individuals with DM2, IGM or insulin resistance are particularly sensitive to the adverse cardiovascular effects of endothelial dysfunction.

\section{Perspectives}

In the pathogenesis of cardiovascular events, true interaction between risk factors has rarely been identified. The present study shows, for the first time, the presence of interaction (i.e. synergy) between endothelial dysfunction and DM2, IGM and insulin resistance with respect to $\mathrm{CV}$ event risk. This suggests that endothelial dysfunction is a key therapeutic target for the prevention of CV events in individuals with DM2, IGM or insulin resistance. 


\section{References}

1. Di Angelantonio E, Gao P, Pennells L, et al. Lipid-related markers and cardiovascular disease prediction. JAMA. 2012;307:2499-2506.

2. Kaptoge S, Di Angelantonio E, Lowe G, et al. C-reactive protein concentration and risk of coronary heart disease, stroke, and mortality: an individual participant meta-analysis. Lancet. 2010;375:132-140.

3. Lewington S, Clarke R, Qizilbash N, Peto R, Collins R. Age-specific relevance of usual blood pressure to vascular mortality: a meta-analysis of individual data for one million adults in 61 prospective studies. Lancet. 2002;360:1903-1913.

4. de Jager J, Dekker JM, Kooy A, et al. Endothelial dysfunction and low-grade inflammation explain much of the excess cardiovascular mortality in individuals with type 2 diabetes: the Hoorn Study. Arterioscler Thromb Vasc Biol. 2006;26:1086-1093.

5. Frankel DS, Meigs JB, Massaro JM, et al. Von Willebrand factor, type 2 diabetes mellitus, and risk of cardiovascular disease: the framingham offspring study. Circulation. 2008;118:2533-2539.

6. Kim JA, Montagnani M, Koh KK, Quon MJ. Reciprocal relationships between insulin resistance and endothelial dysfunction: molecular and pathophysiological mechanisms. Circulation. 2006;113:18881904.

7. Muris DM, Houben AJ, Schram MT, Stehouwer CD. Microvascular dysfunction is associated with a higher incidence of type 2 diabetes mellitus: a systematic review and meta-analysis. Arterioscler Thromb Vasc Biol. 2012;32:3082-3094.

8. $\mathrm{Xu} \mathrm{J,} \mathrm{Zou} \mathrm{MH.} \mathrm{Molecular} \mathrm{insights} \mathrm{and} \mathrm{therapeutic} \mathrm{targets} \mathrm{for} \mathrm{diabetic} \mathrm{endothelial} \mathrm{dysfunction.}$ Circulation. 2009;120:1266-1286.

9. Paneni F, Beckman JA, Creager MA, Cosentino F. Diabetes and vascular disease: pathophysiology, clinical consequences, and medical therapy: part I. Eur Heart J. 2013;34:2436-2443.

10. Ford ES, Zhao G, Li C. Pre-diabetes and the risk for cardiovascular disease: a systematic review of the evidence. J Am Coll Cardiol. 2010;55:1310-1317.

11. Sarwar N, Sattar N, Gudnason V, Danesh J. Circulating concentrations of insulin markers and coronary heart disease: a quantitative review of 19 Western prospective studies. Eur Heart J. 2007;28:2491-2497.

12. Knol MJ, van der Tweel I, Grobbee DE, Numans ME, Geerlings MI. Estimating interaction on an additive scale between continuous determinants in a logistic regression model. Int J Epidemiol. 2007;36:11111118.

13. Rothman KJ, Greenland S, Lash TJ. Modern Epidemiology. Philadelphia: Lippincott Williams And Wilkins; 2008.

14. Henry RM, Ferreira I, Kostense PJ, et al. Type 2 diabetes is associated with impaired endotheliumdependent, flow-mediated dilation, but impaired glucose metabolism is not; The Hoorn Study. Atherosclerosis. 2004;174:49-56.

15. Spijkerman AM, Adriaanse MC, Dekker JM, et al. Diabetic patients detected by population-based stepwise screening already have a diabetic cardiovascular risk profile. Diabetes Care. 2002;25:17841789.

16. Unwin N, Shaw J, Zimmet P, Alberti KG. Impaired glucose tolerance and impaired fasting glycaemia: the current status on definition and intervention. Diabet Med. 2002;19:708-723.

17. van Bussel BC, Henry RM, Schalkwijk CG, Dekker JM, Nijpels G, Stehouwer CD. Low-grade inflammation, but not endothelial dysfunction, is associated with greater carotid stiffness in the elderly: the Hoorn Study. J Hypertens. 2012;30:744-752.

18. Wallace TM, Levy JC, Matthews DR. Use and abuse of HOMA modeling. Diabetes Care. 2004;27:14871495.

19. Clark MG, Wallis MG, Barrett EJ, et al. Blood flow and muscle metabolism: a focus on insulin action. Am J Physiol Endocrinol Metab. 2003;284:E241-258. 
20. Tal MG. Type 2 diabetes: Microvascular ischemia of pancreatic islets? Med Hypotheses. 2009;73:357358.

21. Ceriello A, Esposito K, Piconi L, et al. Oscillating glucose is more deleterious to endothelial function and oxidative stress than mean glucose in normal and type 2 diabetic patients. Diabetes. 2008;57:13491354.

22. Wang J, Alexanian A, Ying R, et al. Acute exposure to low glucose rapidly induces endothelial dysfunction and mitochondrial oxidative stress: role for AMP kinase. Arterioscler Thromb Vasc Biol. 2012;32:712720. 


\section{Supplemental Material}

\section{Supplemental methods}

\section{Brachial artery flow-mediated dilatation}

The measurement protocol has been described in detail. ${ }^{1,2}$ All individuals underwent the ultrasound examination according to the guidelines of the International Brachial Artery Reactivity Task Force. ${ }^{3}$ All measurements were done by one investigator. The diameter of the right brachial artery ( $20 \mathrm{~mm}$ proximal to the antecubital fossa) was assessed with use of an ultrasound scanner equipped with a $7.5 \mathrm{MHz}$ linear probe (350 series, Pie Medical, Maastricht, the Netherlands). The scanner was connected to a PC equipped with vessel wall movement detection software and an acquisition system (Wall Track System, Pie Medical, Maastricht, the Netherlands). This set-up enables measurement of artery diameter, as described previously. ${ }^{4,5}$ Blood flow (peak systolic velocity) was estimated by pulsed-wave Doppler from a sample volume in the centre of the artery at a $60^{\circ}$ angle. To secure the ultrasound image and measurement position throughout the study, we used a stereotactic probe-holding device, while the subject's arm was positioned over a foam cast to inhibit movement. Baseline diameter and peak flow velocity were determined. A pressure cuff, placed on the forearm, was then automatically inflated and kept constant at supra-systolic pressure (brachial systolic pressure $+100 \mathrm{mmHg}$ ) in order to induce forearm ischemia. After $5 \mathrm{~min}$ the cuff was released, which was followed by an increase in blood flow. This increase in blood flow increased shear stress, which served as the stimulus for FMD. After cuff release, maximum peak flow velocity was measured within $15 \mathrm{~s}$, and diameter at 45, 90, 180 and $300 \mathrm{~s}$. The maximum diameter in any of these four measurements was used in the statistical analysis. In addition, endothelium-independent, nitroglycerin-mediated dilatation (NMD) was determined. After 15 min of rest to re-establish baseline conditions, baseline diameter (mean of three measurements) and peak flow velocity (mean of two measurements) were re-determined. Nitroglycerin (400 $\mu \mathrm{g}$, Nitrolingual Spray, PohlBoskamp, Germany) was then sublingually administered; after $5 \mathrm{~min}$, diameter (mean of three measurements) and peak flow velocity (mean of two measurements) were again determined.

Reproducibility was assessed in ten individuals (five men; $58.2 \pm 9.5$ years) whom were examined twice, two weeks apart. The intra-observer intersession coefficients of variation were $4.3 \%$ for diameter, $14.7 \%$ for FMD and $10.3 \%$ for NMD (the latter two expressed as absolute diameter change). 


\section{4 | Chapter 2}

Table S2.2. Association between flow-mediated dilatation and all-cause mortality: stratified (A), and interaction (B) analyses with glucose metabolism and insulin resistance status

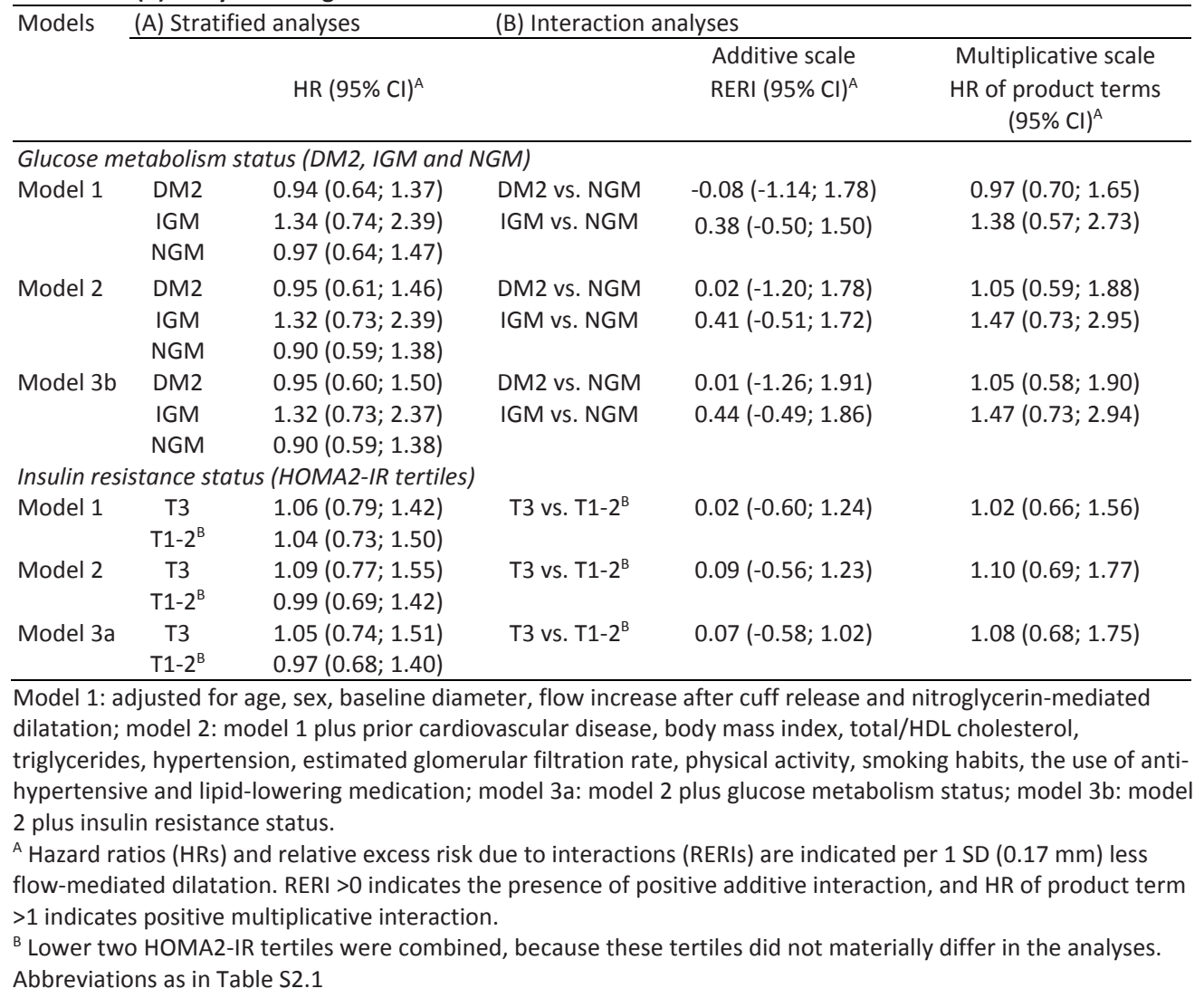


Endothelial dysfunction, diabetes and cardiovascular events | $\mathbf{3 5}$ 


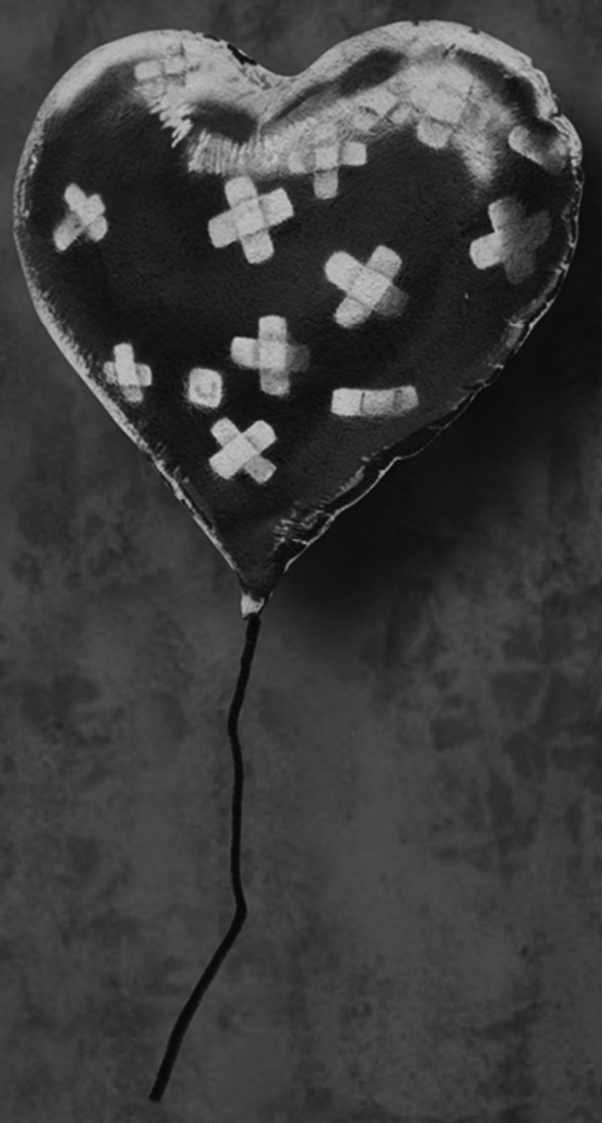

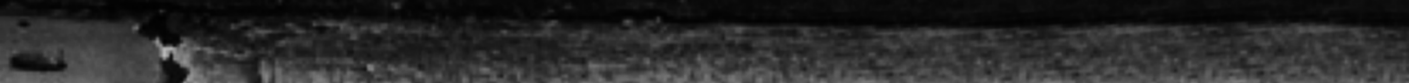
1. 1. 1. 


\section{Chapter 3}

\section{Local stiffness of the carotid and}

femoral artery is associated with

incident cardiovascular disease and allcause mortality: The Hoorn Study

TT van Sloten, MT Schram, K van den Hurk, JM Dekker, G Nijpels, RMA Henry, CDA Stehouwer 
38 | Chapter 3

\begin{abstract}

\section{Background}

The association of different stiffness indices, in particular of carotid, brachial and femoral stiffness, with cardiovascular disease and mortality is currently unknown. This study therefore sought to investigate the association of local and segmental arterial stiffness with incident cardiovascular events and all-cause mortality.
\end{abstract}

\title{
Methods
}

In a population-based cohort ( $n=579$; mean age 67 years; $50 \%$ women; $23 \%$ with type 2 diabetes (by design)), we assessed local stiffness of carotid, femoral and brachial arteries (by ultrasonography), carotid-femoral pulse wave velocity (cfPWV), aortic augmentation index and systemic arterial compliance.

\section{Results}

After a median follow-up of 7.6 years, 130 participants had had a cardiovascular event and 96 had died. Hazard ratios (95\% confidence intervals) per 1 standard deviation for cardiovascular events and all-cause mortality, respectively, were: 1.22 (0.95 to 1.56) and 1.51 (1.11 to 2.06 ) for lower carotid distensibility; 1.19 (1.00 to 1.41 ) and 1.28 (1.07 to 1.53 ) for higher carotid elastic modulus; 1.08 (0.88 to 1.31 ) and 1.43 (1.10 to 1.86) for lower carotid compliance; 1.39 (1.06 to 1.83 ) and 1.27 (0.90 to 1.79) for lower femoral distensibility; 1.25 (0.96 to 1.63 ) and 1.47 (1.01 to 2.13) for lower femoral compliance; and 1.56 (1.23 to 1.98 ) and 1.13 (0.83 to 1.54 ) for higher cfPWV. These results were adjusted for age, sex, mean arterial pressure and cardiovascular risk factors. Mutual adjustments for each of the other stiffness indices did not materially change these results. Brachial stiffness, augmentation index and systemic arterial compliance were not associated with cardiovascular events or mortality.

\section{Conclusion}

Carotid and femoral stiffness indices are independently associated with incident cardiovascular events and all-cause mortality. The strength of these associations with events may differ per stiffness parameter. 


\section{Introduction}

Stiffening of the arterial vasculature leads to increased systolic pressure, decreased coronary perfusion and an increased pulsatile load on the microcirculation. Thereby, arterial stiffness can cause stroke, coronary heart disease (CHD) and heart failure. ${ }^{1,2}$ Stiffness can be measured at different arterial segments or sites, and by use of different techniques. These include segmental carotid-femoral pulse wave velocity (cfPWV); local carotid, femoral or brachial artery stiffness; and assessment of systemic arterial compliance (SAC). ${ }^{1}$ In addition, aortic augmentation index (Aix) is used as a surrogate for wave reflections. ${ }^{1}$

Measurement of arterial stiffness at different sites is important, as stiffness is not uniform along the arterial tree. For example, there are substantial differences in properties between elastic and muscular arteries. Stiffening of elastic and muscular arteries may cause cardiovascular disease (CVD) via different mechanisms, ${ }^{1,2}$ and, therefore, may be differentially associated with CV events and mortality. However, the association of stiffening of different parts of the arterial tree with incident CV events or mortality has not yet been investigated.

Previous studies ${ }^{3,4}$ have shown an independent association of cfPWV with incident CVD. CfPWV, however, reflects the properties of a mixed elastic and muscular part of the arterial tree; thus, cfPWV does not discriminate between these segments. ${ }^{1}$ In contrast, local distensibility measurements of the carotid (a predominantly elastic artery) and the femoral and brachial arteries (predominantly muscular arteries) enable the study of stiffening of elastic and muscular sites. ${ }^{1,2}$ To date, no study has evaluated the association of local stiffness of the femoral and brachial artery and CV events or mortality, and prospective studies on local carotid stiffness are scarce. Some of these studies reported an association between carotid stiffness and incident $C V D^{5-7}$ and/or mortality ${ }^{7}$, whereas others ${ }^{8-12}$ did not. However, most studies (but not all ${ }^{5}$ ) were relatively small $6,7,11,12$ and/or had a relatively short follow-up ( $<5$ years). ${ }^{7-11}$ In addition, previous studies ${ }^{5-8,11,12}$ used brachial derived pulse pressure (PP) to calculate distensibility coefficients. The use of brachial instead of local PP may underestimate the predictive value of carotid stiffness due PP amplification (i.e. the increase in PP along the arterial tree). ${ }^{1,2}$ The magnitude of amplification, however, diminishes with age. ${ }^{1,2}$ Consequently, it has been suggested that, in elderly populations, ${ }^{9-12}$ local stiffness indices calculated with brachial or local PP may yield similar results. This, however, has not yet been investigated.

In view of the above, we investigated the association of, on the one hand, local stiffness of the carotid, brachial and femoral artery, segmental stiffness of the aorta, Aix and SAC with, on the other hand, incident CV events and all-cause mortality, during a median follow-up of 7.6 years in a population-based study of elderly individuals (The Hoorn Study). We 
40 | Chapter 3

additionally investigated whether the associations for local stiffness were different when calibrated local PP instead of brachial PP was used.

\section{Methods}

\section{Study design}

For the present study, we used data from The 2000 Hoorn Study examination $(n=648)$. The Hoorn Study is a population-based cohort study of glucose metabolism and CVD risk among the inhabitants of the municipality of Hoorn in the Netherlands. Details of the study have been described elsewhere. ${ }^{13,14}$

\section{Local stiffness indices of the carotid, femoral and brachial arteries}

Carotid, femoral and brachial arterial properties were determined according to international guidelines. ${ }^{13} \mathrm{~A}$ detailed description of the assessment of local arterial stiffness is provided in the supplemental material. Local arterial stiffness indices were calculated according to the following formulas ${ }^{15}$ :

- $\quad$ Distensibility Coefficient $(D C)=\left(2 \Delta D \times D+\Delta D^{2}\right) /\left(P P \times D^{2}\right)$

- Young's elastic modulus (YEM) (carotid artery only) = D / (IMT x DC)

- $\quad$ Compliance Coefficient $(C C)=\pi \times\left(2 D \times \Delta D+\Delta D^{2}\right) / 4 P P$

Where $\mathrm{D}$ is arterial diameter; $\Delta \mathrm{D}$ is distension; IMT is intima-media thickness; and PP is brachial pulse pressure (calculated as systolic minus diastolic blood pressure). DC represents arterial stiffness; YEM represents the stiffness of the arterial wall material at operating pressure; and CC represents arterial buffering capacity.

\section{CfPWV, Aix, SAC}

CfPWV, that is, the ratio of travelled distance divided by transit time, was estimated using body height (to estimate the travel distance ${ }^{16}$ ) and continuous measurement of the distension curves of the carotid and femoral artery (to estimate transit time ${ }^{14}$ ). The Aix was determined by radial applanation tonometry (Sphygmocor, Atcor Medical, Sydney, Australia). ${ }^{1,14}$ SAC was determined according to two methods: the exponential decay method based on the Windkessel method, and the ratio of stroke volume to aortic PP. ${ }^{14} \mathrm{~A}$ detailed description of the assessment of cfPWV, Aix and SAC is provided in the supplemental material. 


\section{Other measurements}

CVD risk factors were assessed as described previously. ${ }^{13,14}$ Physical activity was assessed by questionnaire. ${ }^{17}$

\section{Follow-up}

Follow-up was complete as of January 1, 2009. Information on morbidity was extracted from individuals' medical records from their general practitioners and from the local hospital, and classified according the International Classification of Disease ( $9^{\text {th }}$ edition; ICD-9). We defined incident CV events (non-fatal and fatal combined) as ICD-9 codes: 410 to 414 (CHD), 427 and 428 (heart failure), 431 to 438 (cerebrovascular disease), 440 to 443 (arterial disease), 798 (sudden death), and ICD-9 clinical modification code 36 (coronary arterial procedures). Data on the participants' vital status were collected from the municipal population register of Hoorn. The cause of death was extracted from the medical records of the general practitioners and the hospital of Hoorn. Information on cause of death could not be obtained for 21 (22\%) of the deceased participants.

\section{Statistical analysis}

All analyses were performed with PASW Statistics (version 21, IBM, Chicago, Illinois, USA). Characteristics of the study population at baseline were compared between participants with and without incident CV events, and between those who did and did not survive, with the use of Student $t$ test or Mann-Whitney $U$ test for continuous variables, and a chi-square test for discrete variables. Cox proportional hazard models were used to estimate the associations between, on the one hand, arterial stiffness indices, and, on the other hand, incident CV events and all-cause mortality. The associations were first adjusted for the stratification variables of The Hoorn Study cohort: age, sex and glucose metabolism ${ }^{13}$ (model 1); additionally for MAP (model 2); and for CVD risk factors: prior CVD, body mass index (BMI), triglycerides, total/high-density lipoprotein cholesterol, estimated glomerular filtration rate, (micro)albuminuria, physical activity and smoking habits (model 3). CfPWV, Aix, and SAC were additionally adjusted for heart rate. Finally, mutual adjustments were made for each of the individual stiffness indices. The associations are given as standardized hazard ratios (HRs) and corresponding 95\% confidence intervals ( $\mathrm{Cls}$ ). We used interaction terms to explore whether any association differed according to sex, prior CVD and/or glucose metabolism status. ${ }^{13,18}$

We tested 12 hypotheses (i.e. whether, on the one hand, local carotid, femoral and brachial stiffness, cfPWV, Aix and SAC, are associated with, on the other, CV events and mortality), each at the .05 level. All other tests (i.e. interactions and additional analyses) were considered to be hypothesis generating and were also conducted at the .05 or, for interaction analyses, .10 level. 
42 | Chapter 3

\section{Results}

\section{Study population}

For the present analyses, all participants were included in whom data were available on the carotid, femoral or brachial stiffness indices, cfPWV, Aix or SAC. Figure 3.1 shows the number of participants per stiffness measurement. The main reason for missing data on local stiffness indices was poor definition of the arterial wall due to obesity (the BMI of those with qualitatively sufficient examinations vs. those without was $26.4 \pm 3.1 \mathrm{~kg} / \mathrm{m}^{2}$ vs. $30.4 \pm$ $5.2 \mathrm{~kg} / \mathrm{m}^{2}$ ). Missing data on Aix and SAC was due to device availability, which was not related to the clinical status of the participants. Missing data on cfPWV was due to technical reasons (i.e. no qualitatively acceptable distension curve available for both the carotid and femoral artery), as well as due to later addition of the (automatic) calculation of carotid-femoral transit time to the vascular ultrasound protocol than the local stiffness measurements.

Participants were excluded when data on glucose metabolism status $(n=10)$ or follow-up were missing. CV event follow-up was missing for 46 participants either because they had moved out of the town of Hoorn and could not be contacted $(n=7)$, or because they had not given permission to access their medical files $(n=39)$. Individuals without follow-up data did not differ from the study population (data not shown). None of the participants were lost to follow-up for all-cause mortality. 


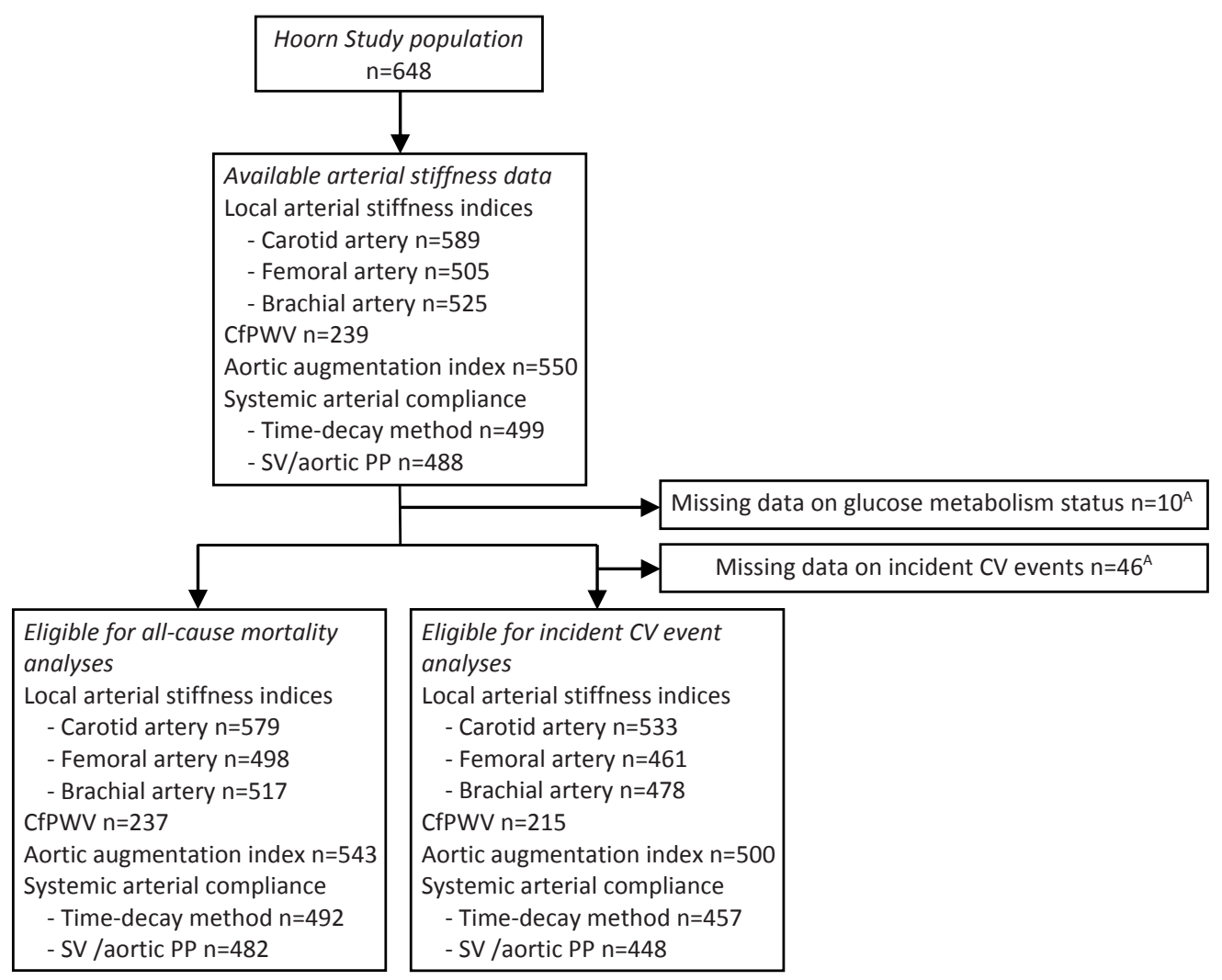

Figure 3.1. Flow diagram of the study.

A Number varies per measurement, maximum number of missing values indicated.

cfPWV = carotid-femoral pulse wave velocity; SV = stroke volume; PP = pulse pressure; $\mathrm{CV}=$ cardiovascular.

\section{Clinical characteristics}

Table 3.1 shows baseline characteristics of the study population according to CV event and mortality status. A total of 130 participants had a CV event (non-fatal and fatal combined) (median follow-up 7.6 years, [range 0.2 to 8.9 years), of whom 40 had a cerebrovascular event, 58 had a CHD event (7 persons had both a cerebrovascular and a CHD event), and 39 had a CV event other than a cerebrovascular or CHD event (e.g. peripheral arterial disease or heart failure). In addition, 96 participants died (median follow-up 7.8 years [range 0.02 to 8.9 years]), of whom 22 (23\%) died of a CVD, 31 (32\%) of cancer, 22 (23\%) of other causes and $21(22 \%)$ of causes unknown. The incidence rates per year were $3.1 \%$ for CV events, $1.4 \%$ for CHD events, $1.0 \%$ for cerebrovascular events and $2.1 \%$ for all-cause mortality. 
Table 3.1. Clinical characteristics of the study population at baseline according to cardiovascular event and mortality status ${ }^{\mathrm{A}}$

\begin{tabular}{|c|c|c|c|c|}
\hline & $\begin{array}{c}\text { Participants } \\
\text { without incident } \\
\text { CV event } \\
n=403(75.6 \%)\end{array}$ & $\begin{array}{l}\text { Participants } \\
\text { with incident } \\
\text { CV event } \\
n=130(24.4 \%)\end{array}$ & $\begin{array}{c}\text { Survivors } \\
\mathrm{n}=483(83.4 \%)\end{array}$ & $\begin{array}{c}\text { Deceased } \\
n=96(16.6 \%)\end{array}$ \\
\hline \multicolumn{5}{|l|}{ General characteristics } \\
\hline Women & 54.8 & 35.4 & 53.4 & 36.5 \\
\hline Age (years) & $69.0 \pm 6.4$ & $71.9 \pm 6.2$ & $68.9 \pm 6.0$ & $73.3 \pm 6.9$ \\
\hline \multicolumn{5}{|l|}{ Smoking habits } \\
\hline Current smoker & 11.7 & 22.3 & 13.7 & 20.8 \\
\hline Former smoker & 44.3 & 51.5 & 44.2 & 51.0 \\
\hline Non-smoker & 44.0 & 26.2 & 42.1 & 28.1 \\
\hline Physical activity (MET h/week) & $82(47-127)$ & $69(41-125)$ & $82(50-130)$ & $69(30-121)$ \\
\hline Prior cardiovascular disease & 47.0 & 63.8 & 49.1 & 60.9 \\
\hline \multicolumn{5}{|l|}{ Glucose metabolism status } \\
\hline Type 2 diabetes mellitus & 22.1 & 25.8 & 19.7 & 39.6 \\
\hline Impaired glucose metabolism & 27.5 & 34.8 & 29.0 & 29.2 \\
\hline Normal glucose metabolism & 50.4 & 39.4 & 51.3 & 31.3 \\
\hline Body mass index $\left(\mathrm{kg} / \mathrm{m}^{2}\right)$ & $27.0 \pm 3.6$ & $27.0 \pm 3.4$ & $27.0 \pm 3.6$ & $27.2 \pm 3.5$ \\
\hline Systolic blood pressure (mmHg) & $140 \pm 20$ & $148 \pm 21$ & $141 \pm 20$ & $147 \pm 21$ \\
\hline Diastolic blood pressure (mmHg) & $82 \pm 11$ & $83 \pm 12$ & $83 \pm 11$ & $83 \pm 12$ \\
\hline Hypertension (\%) & 62.9 & 80.6 & 65.1 & 77.9 \\
\hline $\mathrm{HbA1c}$ (mmol/mol) & $42 \pm 8$ & $44 \pm 8$ & $41 \pm 7$ & $46 \pm 9$ \\
\hline HbA1c (\%) & $5.9 \pm 0.7$ & $6.2 \pm 0.8$ & $5.9 \pm 0.7$ & $6.3 \pm 0.9$ \\
\hline Total cholesterol (mmol/L) & $5.7 \pm 1.0$ & $5.7 \pm 1.1$ & $5.8 \pm 1.4$ & $5.6 \pm 1.0$ \\
\hline LDL cholesterol (mmol/L) & $3.6 \pm 0.9$ & $3.7 \pm 0.9$ & $3.7 \pm 0.9$ & $3.6 \pm 0.9$ \\
\hline HDL cholesterol (mmol/L) & $1.5 \pm 0.4$ & $1.3 \pm 0.4$ & $1.5 \pm 0.4$ & $1.3 \pm 0.4$ \\
\hline Triglycerides (mmol/L) & $1.2(0.9-1.7)$ & $1.4(1.1-1.8)$ & $1.3(1.0-1.7)$ & $1.5(1.1-1.9)$ \\
\hline eGFR $\left(\mathrm{ml} / \mathrm{min} / 1.73 \mathrm{~m}^{2}\right)$ & $62.5 \pm 10.3$ & $60.4 \pm 11.6$ & $63 \pm 10.0$ & $59 \pm 12.5$ \\
\hline $\begin{array}{l}\text { (Micro)albuminuria (albumin /creatinine } \\
\text { ratio }>2 \mathrm{mg} / \mathrm{mmol} \text { ) }\end{array}$ & 11.4 & 21.7 & 11.6 & 26.0 \\
\hline Lipid-lowering medication & 14.4 & 20.0 & 15.6 & 16.7 \\
\hline Anti-hypertensive medication & 31.3 & 46.9 & 34.9 & 46.9 \\
\hline \multicolumn{5}{|l|}{ Arterial stiffness indices } \\
\hline \multicolumn{5}{|l|}{ Carotid artery } \\
\hline Distensibility coefficient $\left(10^{-3} / \mathrm{kPa}\right)$ & $11.2 \pm 4.2$ & $9.7 \pm 4.0$ & $11.3 \pm 4.1$ & $8.6 \pm 3.8$ \\
\hline Young's elastic modulus $\left(10^{3} \mathrm{x} \mathrm{kPa}\right)$ & $0.98 \pm 0.46$ & $1.20 \pm 0.84$ & $0.96 \pm 0.46$ & $1.36 \pm 0.89$ \\
\hline Compliance coefficient $\left(\mathrm{mm}^{2} / \mathrm{kPa}\right)$ & $0.53 \pm 0.21$ & $0.52 \pm 0.23$ & $0.54 \pm 0.22$ & $0.45 \pm 0.17$ \\
\hline \multicolumn{5}{|l|}{ Femoral artery } \\
\hline Distensibility coefficient $\left(10^{-3} / \mathrm{kPa}\right)$ & $5.4 \pm 2.4$ & $4.4 \pm 1.8$ & $5.3 \pm 2.4$ & $4.1 \pm 2.0$ \\
\hline Compliance coefficient $\left(\mathrm{mm}^{2} / \mathrm{kPa}\right)$ & $0.43 \pm 0.22$ & $0.37 \pm 0.17$ & $0.43 \pm 0.21$ & $0.32 \pm 0.17$ \\
\hline \multicolumn{5}{|l|}{ Brachial artery } \\
\hline Distensibility coefficient $\left(10^{-3} / \mathrm{kPa}\right)$ & $7.9 \pm 4.3$ & $7.9 \pm 4.2$ & $7.9 \pm 4.2$ & $7.4 \pm 4.1$ \\
\hline Compliance coefficient (mm²/kPa) & $0.13 \pm 0.07$ & $0.14 \pm 0.08$ & $0.13 \pm 0.07$ & $0.13 \pm 0.07$ \\
\hline Carotid-femoral PWV (m/s) & $10.0 \pm 3.5$ & $12.4 \pm 5.9$ & $9.7 \pm 3.5$ & $12.4 \pm 6.0$ \\
\hline Aortic augmentation index (\% point) & $32 \pm 9$ & $33 \pm 9$ & $32 \pm 9$ & $33 \pm 9$ \\
\hline \multicolumn{5}{|l|}{ Systemic arterial compliance $(\mathrm{ml} / \mathrm{mmHg})$} \\
\hline Time-decay method & $0.74 \pm 0.31$ & $0.71 \pm 0.33$ & $0.74 \pm 0.31$ & $0.66 \pm 0.33$ \\
\hline SV /aortic PP & $1.07 \pm 0.35$ & $1.02 \pm 0.36$ & $1.07 \pm 0.34$ & $0.97 \pm 0.36$ \\
\hline
\end{tabular}

Values are $\%$, mean \pm SD or median (interquartile range).

A Numbers correspond with the number of participants with available carotid artery ultrasound data.

$\mathrm{CV}=$ cardiovascular; eGFR = estimated glomerular filtration rate; $\mathrm{HbA1c}=$ glycosylated hemoglobin; $\mathrm{HDL}=\mathrm{high}-$ density lipoprotein; $L D L=$ low-density lipoprotein; $M E T$ = metabolic equivalent of task; $P P=$ pulse pressure; $\mathrm{PWV}=$ pulse wave velocity; SV = stroke volume. 


\section{Incident CV events}

A

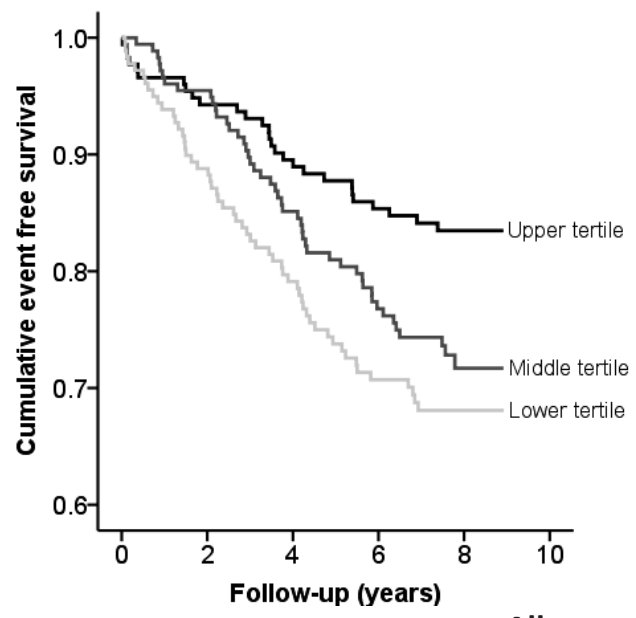

All-cause mortality

C

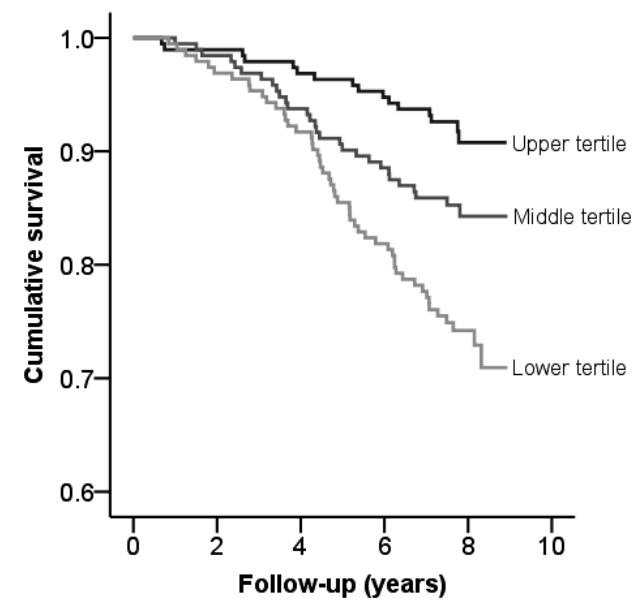

D
B

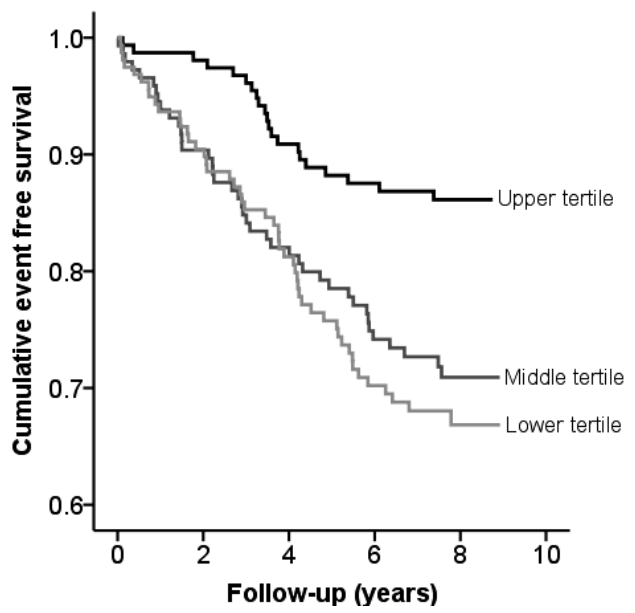

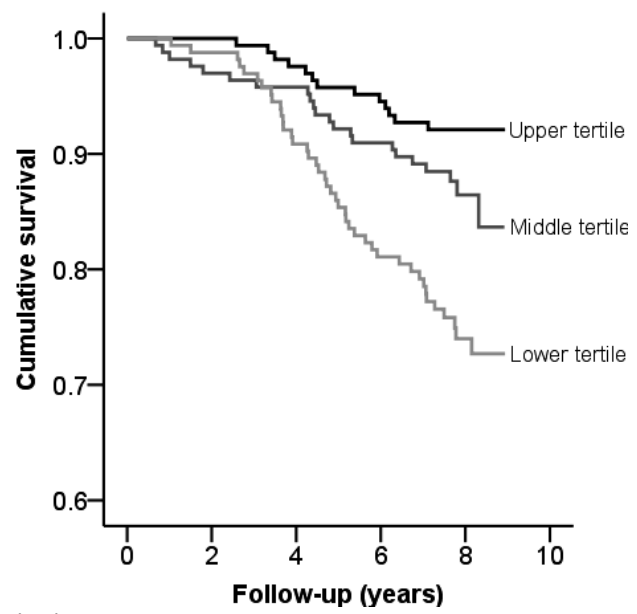

Figure 3.2. Kaplan-Meier curves for incident cardiovascular (CV) events and all-cause mortality according to tertiles of distensibility coefficients (DC) of the carotid ( $A$ and $C$ ) and femoral artery (B and D).

\section{Local arterial stiffness indices and incident $\mathrm{CV}$ events}

Figure 3.2 shows Kaplan-Meier curves for incident CV events according to tertiles of the carotid (panel A) and femoral DC (panel B). Cox regression analyses adjusted for age, sex and glucose metabolism showed that lower carotid DC, higher YEM and lower femoral DC and $\mathrm{CC}$ were associated with a higher $\mathrm{CV}$ event incidence (Table 3.2, model 1). The carotid CC and the brachial DC and CC were not associated with incident CV events (Table 3.2, model 1). Further adjustments for MAP (model 2) and CVD risk factors (model 3) did not materially change the associations between YEM and femoral DC, on the one hand, and incident $\mathrm{CV}$ events, on the other, whereas the association was attenuated for the carotid 
DC and the femoral CC. In addition, the associations between the carotid, femoral and brachial DC or CC and incident CV events did not materially change after mutual adjustments for each of the other stiffness indices (for adjustments for cfPWV, see supplemental material, Table S3.1).

\section{Local stiffness indices and all-cause mortality}

Figure 3.2 shows Kaplan-Meier curves for all-cause mortality according to tertiles of the carotid (panel C) and femoral DC (panel D). Cox regression analyses adjusted for age, sex and glucose metabolism showed that lower carotid DC and CC, higher YEM and lower femoral DC and CC were associated with greater all-cause mortality (Table 3.2, model 1). Brachial DC and CC were not associated with all-cause mortality (Table 3.2, model 1). Further adjustments for MAP (model 2) and CVD risk factors (model 3) did not materially change the associations between carotid DC, CC and YEM and all-cause mortality, whereas the association was attenuated for femoral DC and CC. In addition, the associations between the carotid, femoral and brachial DC or CC and all-cause mortality did not materially change after mutual adjustments for each of the other stiffness indices (for adjustments for cfPWV, see supplemental material, Table S3.1).

Table 3.2. Associations between arterial stiffness indices of the carotid, femoral and brachial artery with incident cardiovascular events and all-cause mortality

\begin{tabular}{|c|c|c|c|c|c|c|c|}
\hline \multirow[t]{2}{*}{ Model } & \multicolumn{3}{|c|}{ Carotid artery } & \multicolumn{2}{|c|}{ Femoral artery } & \multicolumn{2}{|c|}{ Brachial artery } \\
\hline & DC & YEM & $\mathrm{CC}$ & $\mathrm{DC}$ & $\mathrm{CC}$ & $\mathrm{DC}$ & $\mathrm{CC}$ \\
\hline \multicolumn{8}{|c|}{ Incident cardiovascular events } \\
\hline 1 & $\begin{array}{c}1.27 \\
(1.02 ; 1.57)\end{array}$ & $\begin{array}{c}1.22 \\
(1.04 ; 1.42)\end{array}$ & $\begin{array}{c}1.07 \\
(0.88 ; 1.30)\end{array}$ & $\begin{array}{c}1.39 \\
(1.07 ; 1.81)\end{array}$ & $\begin{array}{c}1.29 \\
(0.99 ; 1.67)\end{array}$ & $\begin{array}{c}0.90 \\
(0.74 ; 1.08)\end{array}$ & $\begin{array}{c}0.84 \\
(0.71 ; 1.01)\end{array}$ \\
\hline 2 & $\begin{array}{c}1.24 \\
(0.98 ; 1.58)\end{array}$ & $\begin{array}{c}1.20 \\
(1.02 ; 1.42)\end{array}$ & $\begin{array}{c}1.04 \\
(0.86 ; 1.28)\end{array}$ & $\begin{array}{c}1.37 \\
(1.04 ; 1.80)\end{array}$ & $\begin{array}{c}1.26 \\
(0.97 ; 1.65)\end{array}$ & $\begin{array}{c}0.87 \\
(0.72 ; 1.06)\end{array}$ & $\begin{array}{c}0.84 \\
(0.70 ; 1.00)\end{array}$ \\
\hline 3 & $\begin{array}{c}1.22 \\
(0.95 ; 1.56)\end{array}$ & $\begin{array}{c}1.19 \\
(1.00 ; 1.41)\end{array}$ & $\begin{array}{c}1.08 \\
(0.88 ; 1.31)\end{array}$ & $\begin{array}{c}1.39 \\
(1.06 ; 1.83)\end{array}$ & $\begin{array}{c}1.25 \\
(0.96 ; 1.63)\end{array}$ & $\begin{array}{c}0.88 \\
(0.72 ; 1.07)\end{array}$ & $\begin{array}{c}0.85 \\
(0.71 ; 1.02)\end{array}$ \\
\hline \multicolumn{8}{|c|}{ All-cause mortality } \\
\hline 1 & $\begin{array}{c}1.62 \\
(1.24 ; 2.13)\end{array}$ & $\begin{array}{c}1.33 \\
(1.15 ; 1.55)\end{array}$ & $\begin{array}{c}1.50 \\
(1.16 ; 1.95)\end{array}$ & $\begin{array}{c}1.35 \\
(0.97 ; 1.88)\end{array}$ & $\begin{array}{c}1.59 \\
(1.11 ; 2.28)\end{array}$ & $\begin{array}{c}0.98 \\
(0.77 ; 1.25)\end{array}$ & $\begin{array}{c}1.06 \\
(0.84 ; 1.33)\end{array}$ \\
\hline 2 & $\begin{array}{c}1.52 \\
(1.13 ; 2.05)\end{array}$ & $\begin{array}{c}1.27 \\
(1.08 ; 1.50)\end{array}$ & $\begin{array}{c}1.41 \\
(1.08 ; 1.84)\end{array}$ & $\begin{array}{c}1.27 \\
(0.90 ; 1.78)\end{array}$ & $\begin{array}{c}1.52 \\
(1.06 ; 2.19)\end{array}$ & $\begin{array}{c}0.92 \\
(0.72 ; 1.17)\end{array}$ & $\begin{array}{c}1.02 \\
(0.82 ; 1.28)\end{array}$ \\
\hline 3 & $\begin{array}{c}1.51 \\
(1.11 ; 2.06)\end{array}$ & $\begin{array}{c}1.28 \\
(1.07 ; 1.53)\end{array}$ & $\begin{array}{c}1.43 \\
(1.10 ; 1.86)\end{array}$ & $\begin{array}{c}1.27 \\
(0.90 ; 1.79)\end{array}$ & $\begin{array}{c}1.47 \\
(1.01 ; 2.13) \\
\end{array}$ & $\begin{array}{c}0.90 \\
(0.70 ; 1.15)\end{array}$ & $\begin{array}{c}0.99 \\
(0.78 ; 1.25)\end{array}$ \\
\hline
\end{tabular}

Values are hazard ratio (95\% confidence interval). Hazard ratios are indicated per 1 SD lower distensibility (DC) and compliance coefficient (CC) and per 1 SD higher Young's elastic modulus (YEM). Model 1: adjusted for age, sex and glucose metabolism status; model 2: model 1 plus mean arterial pressure; model 3: model 2 plus prior cardiovascular disease, BMI, triglycerides, total/HDL cholesterol ratio, eGFR, (micro)albuminuria, physical activity and smoking habits.

The number of participants and events available for analysis with incident cardiovascular events / all-cause mortality: for carotid DC, CC and YEM 533 (130 events) / 579 (96 events); for femoral DC and CC 461 (111 events) / 498 (77 events); and for brachial DC and CC 478 (116 events) / 517 (81 events).

Abbreviations as in Table 3.1. 


\section{CfPWV, incident CV events and all-cause mortality}

Higher cfPWV was associated with a higher CV event incidence (Table 3.3, models 1 to 3). In addition, the association between cfPWV and incident CV events did not materially change after adjustments for each of the other stiffness indices (data not shown). CfPWV was not significantly associated with all-cause mortality (Table 3.3, models 1 to 3).

\section{Aix, SAC and incident CV events and all-cause mortality}

Aix and SAC, either determined via the time-decay method or by the stroke volume to aortic PP ratio, were not associated with CV events or all-cause mortality (Table 3.3, models 1 to 3).

Table 3.3. Associations between the cfPWV, Aix and systemic arterial compliance with incident cardiovascular events and all-cause mortality

\begin{tabular}{|c|c|c|c|c|}
\hline Model & cfPWV & Aix & $\begin{array}{c}\text { SAC (time-decay } \\
\text { method) }\end{array}$ & SAC (SV/aortic PP) \\
\hline \multicolumn{5}{|c|}{ Incident cardiovascular events } \\
\hline 1 & $1.57(1.29 ; 1.92)$ & $1.08(0.89 ; 1.31)$ & $1.09(0.88 ; 1.35)$ & $1.15(0.90 ; 1.47)$ \\
\hline 2 & $1.56(1.27 ; 1.93)$ & $1.05(0.86 ; 1.28)$ & $1.03(0.81 ; 1.30)$ & $1.09(0.84 ; 1.43)$ \\
\hline 3 & $1.56(1.23 ; 1.98)$ & $0.99(0.81 ; 1.22)$ & $1.00(0.79 ; 1.26)$ & $1.13(0.87 ; 1.47)$ \\
\hline \multicolumn{5}{|c|}{ All-cause mortality } \\
\hline 1 & $1.27(0.99 ; 1.63)$ & $1.05(0.84 ; 1.32)$ & $1.22(0.92 ; 1.61)$ & $1.05(0.70 ; 1.58)$ \\
\hline 2 & $1.18(0.88 ; 1.57)$ & $1.19(0.92 ; 1.54)$ & $1.06(0.78 ; 1.43)$ & $1.05(0.67 ; 1.65)$ \\
\hline 3 & $1.13(0.83 ; 1.54)$ & $0.93(0.73 ; 1.18)$ & $1.04(0.76 ; 1.41)$ & $1.01(0.73 ; 1.41)$ \\
\hline
\end{tabular}

Values are hazard ratio (95\% confidence interval). Hazard ratios are indicated per 1 SD higher carotid-femoral pulse wave velocity (cfPWV) and aortic augmentation index (Aix) and per 1 SD lower systemic arterial compliance (SAC). Model 1: adjusted for age, sex and glucose metabolism status; model 2: model 1 plus mean arterial pressure and heart rate; model 3: model 2 plus prior cardiovascular disease, BMI, triglycerides, total/HDL cholesterol ratio, eGFR, (micro)albuminuria, physical activity and smoking habits.

The number of participants and events available for analysis with incident cardiovascular events / all-cause mortality: for cfPWV 215 (53 events) / 237 (36 events); for Aix 500 (120 events) / 543 (87 events); for systemic arterial compliance (time-decay method) 457 (110 events) / 492 (77 events); and for systemic arterial compliance (SV/aortic PP) 448 (106 events) / 482 (70 events).

Abbreviations as in Table 3.1.

\section{Additional analyses}

Analyses were repeated with distension-waveform-calibrated local PP ${ }^{19}$ in a subsample of the study population (data on carotid and femoral PP were not available in 68 and 8 participants, respectively). The results of these analyses (see supplemental material, Table S3.2) were qualitatively similar to the results that used brachial PP.

We additionally adjusted all analyses for systolic pressure and PP instead of MAP and for IMT. After these adjustments, results did not materially change (see supplemental material, Table S3.3). In addition, further adjustments for the use of anti-hypertensive and/or lipidlowering medication did not materially change the results (data not shown).

Analyses with specific cerebrovascular and CHD events did not show statistically significant associations (see supplemental material, Table S3.4). When we analysed the association 
48 | Chapter 3

between arterial stiffness indices and CV events defined as ICD-9 codes 390 to 459 and 798, results did not materially change (data not shown).

The elastance-wall thickness product of the carotid artery was significantly associated with incident CV events and all-cause mortality (see supplemental material, Table S3.5).

Finally, associations of the stiffness indices with incident CV events and all-cause mortality may differ according to sex, presence of prior CVD, or glucose metabolism status. ${ }^{13,18}$ We found no such interactions ( $P$ for interactions $>.10$ ), except for the associations between brachial CC with CV events. A significant association was present between lower brachial CC and incident CVD in persons with prior CVD (HR per 1 SD: 0.78 [ $95 \% \mathrm{Cl} 0.64$ to 0.94$]$ ), but not in persons without prior CVD (0.90 [0.64 to 1.26]) ( $P$ for interaction $=0.07$ ), after adjustment for all potential confounders. In addition, significant associations between lower brachial DC and CC and incident CV events were present in persons with impaired glucose metabolism (IGM) (0.68 [0.50 to 0.93] and 0.69 [0.51 to 0.93], respectively), and in persons with type 2 diabetes (DM2) (0.68 [0.43 to 1.09] and 0.53 [0.33 to 0.84 ], respectively) but not in persons with normal glucose tolerance (NGM) (0.94 [0.67 to 1.32] and 0.93 [0.70 to 1.24], respectively) ( $P$ for interactions $<.10)$, after adjustment for all potential confounders. 


\section{Discussion}

This population-based study is the first to show an association between local stiffness of both the carotid and femoral artery and incident CV events and all-cause mortality. These associations were independent of age, sex, blood pressure, glucose metabolism and CVD risk factors. Furthermore, the associations between carotid and femoral stiffness indices and incident $\mathrm{CV}$ events and mortality did not materially change after adjustments for each of the other stiffness indices. In addition, cfPWV was associated with a higher CV event incidence, but not with all-cause mortality, whereas Aix, SAC and brachial stiffness were not associated with either incident CV events or mortality.

Our study is in accordance with previous studies which showed an association between cfPWV and CV events ${ }^{3,4}$; therefore, cfPWV can be regarded as an internal validation marker of the present study. In addition, the present study extends these findings as it shows that local carotid and femoral stiffness is also independently associated with incident CV events and mortality. Advantages of the present study are the comprehensive assessment of multiple stiffness indices, both local and segmental and at different arterial sites, the use of both brachial and calibrated local PP to compute local stiffness indices, and the long duration of follow-up.

Currently, two population-based studies ${ }^{5,10}$ have evaluated the association of local carotid stiffness and incident CVD and/or mortality, whereas no studies have evaluated the association of local femoral or brachial stiffness and incident CVD or mortality. The ARIC (Atherosclerosis Risk in Communities) Study ${ }^{5}$ indicated an independent association between carotid stiffness and stroke. The Rotterdam Study ${ }^{10}$ also observed an association between carotid stiffness and incident CVD and all-cause mortality, although this association was attenuated and became statistically nonsignificant after adjustments for potential confounders. This study, however, did not measure concurrent blood pressures (i.e. during the time of the stiffness measurement) which may have led to an underestimation of the observed associations.

\section{Associations of local stiffness with CV events and all-cause mortality}

In the present study, local carotid stiffness indices were more strongly associated with allcause mortality than with incident CV events. This finding is difficult to interpret, and may have several explanations. First, incident CVD is more likely subject to misclassification than is all-cause mortality. Second, arterial stiffness may be a marker of biological aging, ${ }^{2}$ and, thus, may affect the risk of an individual to die of any age-related disease, not just CVD. In the elderly population that we studied, this mechanism may well be operative. Nevertheless, we cannot exclude the play of chance, and this issue needs further study. 


\section{Carotid vs. femoral artery stiffness}

The present study showed that stiffness of the carotid and femoral artery is associated with incident CV events and mortality independently of each other. This may suggest that stiffening of these arteries increases CVD and mortality risk via distinct pathways. The ARIC study ${ }^{5}$ indicated that carotid stiffness is more strongly associated with cerebrovascular disease compared with CHD, possibly because stiffening of this artery (or of other elastic arteries for which this artery might serve as a proxy) leads to a high pressure load on the brain. In contrast, femoral stiffness may be more strongly associated with CHD compared with cerebrovascular disease, because femoral and coronary arteries show similar wall characteristics (i.e. both are muscular vessels ${ }^{2}$ and have a high collagen/elastin ratio ${ }^{20}$ ), and, therefore, stiffening of the femoral artery may serve as a proxy for stiffening of the coronary vasculature. The present study had insufficient power to detect associations with specific CV events, and this issue requires further study.

\section{Clinical relevance}

Carotid and femoral stiffness are potential separate therapeutic targets for CVD risk lowering therapy. CVD risk factors have different impacts on stiffening of carotid vs. femoral arteries. ${ }^{13,18,21}$ This may be attributed to the marked differences in the architecture of elastic vs. muscular arteries, and suggests that stiffness of elastic and muscular arteries may be specifically targeted. Of the current available drugs, organic nitrates lower stiffness of muscular arteries. ${ }^{22}$ No therapy is yet available that specifically targets stiffness of elastic arteries. In addition, carotid and femoral stiffness were associated with CV events and mortality even after adjustments for CVD risk factors, IMT, systolic pressure, PP, and other stiffness indices (including cfPWV) and, therefore, may carry additional value with regard to CVD prediction.

\section{Aix and SAC}

In the present study, Aix and SAC were not associated with incident CV events or mortality. With regard to Aix, this is in contrast to a recent meta-analysis, ${ }^{23}$ in which Aix independently predicted CVD, and studies ${ }^{23,24}$ which showed an association between Aix and CVD in men only (in the present study, no interaction with sex was present). More recent data from the Framingham Heart Study ${ }^{3}$ and MESA (Multi-Ethnic Study of Atherosclerosis), ${ }^{25}$ however, indicated that Aix was not ${ }^{3}$ or weakly ${ }^{25}$ associated with CVD, respectively. In addition to magnitude and timing of wave reflections, Aix is also influenced by hemodynamic phenomena unrelated to wave reflections, ${ }^{2,25}$ and these phenomena may be different in DM2/IGM as compared with NGM. ${ }^{26}$ This may result in an underestimation of the association between wave reflections and CVD. Reflection estimates derived from wave separation analysis may more accurately indicate wave reflections than the Aix. In accordance, recent data of $\mathrm{MESA}^{25}$ showed that these estimates were more strongly associated with CVD than Aix. With regard to SAC, a possible explanation for the lack of an 
association might be that the assessment of this estimate has a relatively large measurement error. ${ }^{1}$

\section{Brachial artery stiffness}

In the total study population, local brachial stiffness was not associated with CV events and mortality. In accordance, a previous study ${ }^{27}$ showed that segmental stiffness of the brachial artery (i.e. brachial artery pulse wave velocity) was not associated with CVD mortality. The lack of this association can be explained by the fact that there is no arterial aging of the brachial artery. ${ }^{21}$ In accordance, in the present study population, the carotid and femoral arteries were stiffer in older participants, whereas brachial stiffness was not associated with age (data not shown). Somewhat surprisingly, however, the results of the interaction analysis showed that, in individuals with, but not in those without prior CVD, lower stiffness of the brachial artery was associated with greater risk of CV events. In addition, a similar association between lower brachial stiffness and incident CVD was present in individuals with DM2 and IGM, but not in individuals with NGM. A plausible underlying explanation for these observations is lacking and these findings may represent the play of chance. Further studies are needed to clarify this issue.

\section{Use of brachial derived PP in the calculation of local stiffness indices}

The present study supports the hypothesis that, in elderly people (i.e. age $>60$ years), calculation of local stiffness indices with brachial derived PP is as accurate as the use of local PP. The use of brachial derived vs. local PP is an important methodological issue in the assessment of local stiffness, because of the phenomenon of PP amplification. ${ }^{1,2}$ This phenomenon, however, may play a less important role in elderly people, as the magnitude of amplification diminishes with age. ${ }^{1,2}$ In accordance, the results of the present analyses were qualitatively similar when brachial or calibrated local PP were used.

\section{Limitations}

First, unavoidable survival bias will, in general, have led to an underestimation of the reported associations. Second, the present study had insufficient power to quantify the additive predictive value of local stiffness beyond standard CVD risk factors. Studies are needed to further clarify this issue. Third, data on cfPWV were available in a subsample of the study population only (cfPWV analysis: $n=237 ; 53 \mathrm{CV}$ events and 36 deaths). This may explain why we did not observe an association between cfPWV and all-cause mortality. To our knowledge, carotid-femoral transit time as determined by distension curves has not been validated against any other method. Nevertheless, it has been shown that this method is highly reproducible $e^{28}$ and the present results are in accordance with studies that used applanation tonometry to determine cfPWV. ${ }^{4}$ Finally, a relatively large number of statistical tests were done. The aim of the present study was to evaluate the prognostic value of multiple stiffness indices, which, as a consequence, involves carrying out multiple tests. The 


\section{2 | Chapter 3}

associations with CV events and mortality, were, however, consistent across the different stiffness indices studied. It is therefore unlikely that these findings are the result of the play of chance.

\section{Conclusions}

The present study shows that local stiffness of the carotid and femoral artery is associated with incident CV events and all-cause mortality, independently of CVD risk factors and each of the other stiffness indices. These data suggest that local carotid and femoral stiffness indices may serve as a target for lowering of CVD risk and can have predictive value. Further studies are needed to quantify the predictive value of local stiffness beyond standard CVD risk factors. 


\section{References}

1. Laurent S, Cockcroft J, Van Bortel L, et al. Expert consensus document on arterial stiffness: methodological issues and clinical applications. Eur Heart J. 2006;27:2588-2605.

2. Safar ME, O'Rourke MF. Arterial stiffness in hypertension. Amsterdam: Elsevier; 2006.

3. Mitchell GF, Hwang SJ, Vasan RS, et al. Arterial stiffness and cardiovascular events: the Framingham Heart Study. Circulation. 2010;121:505-511.

4. Vlachopoulos C, Aznaouridis K, Stefanadis C. Prediction of cardiovascular events and all-cause mortality with arterial stiffness: a systematic review and meta-analysis. J Am Coll Cardiol. 2010;55:1318-1327.

5. Yang EY, Chambless L, Sharrett AR, et al. Carotid arterial wall characteristics are associated with incident ischemic stroke but not coronary heart disease in the Atherosclerosis Risk in Communities (ARIC) study. Stroke. 2012;43:103-108.

6. Barenbrock M, Kosch M, Joster E, Kisters K, Rahn KH, Hausberg M. Reduced arterial distensibility is a predictor of cardiovascular disease in patients after renal transplantation. J Hypertens. 2002;20:79-84.

7. Blacher J, Pannier B, Guerin AP, Marchais SJ, Safar ME, London GM. Carotid arterial stiffness as a predictor of cardiovascular and all-cause mortality in end-stage renal disease. Hypertension. 1998;32:570-574.

8. Dijk JM, Algra A, van der Graaf Y, Grobbee DE, Bots ML. Carotid stiffness and the risk of new vascular events in patients with manifest cardiovascular disease. The SMART study. Eur Heart J. 2005;26:12131220.

9. Leone N, Ducimetiere P, Gariepy J, et al. Distension of the carotid artery and risk of coronary events: the three-city study. Arterioscler Thromb Vasc Biol. 2008;28:1392-1397.

10. Mattace-Raso FU, van der Cammen TJ, Hofman A, et al. Arterial stiffness and risk of coronary heart disease and stroke: the Rotterdam Study. Circulation. 2006;113:657-663.

11. Stork S, van den Beld AW, von Schacky C, et al. Carotid artery plaque burden, stiffness, and mortality risk in elderly men: a prospective, population-based cohort study. Circulation. 2004;110:344-348.

12. van Dijk RA, Dekker JM, Nijpels G, Heine RJ, Bouter LM, Stehouwer CD. Brachial artery pulse pressure and common carotid artery diameter: mutually independent associations with mortality in subjects with a recent history of impaired glucose tolerance. Eur J Clin Invest. 2001;31:756-763.

13. Henry RM, Kostense PJ, Spijkerman AM, et al. Arterial stiffness increases with deteriorating glucose tolerance status: the Hoorn Study. Circulation. 2003;107:2089-2095.

14. Schram MT, Henry RM, van Dijk RA, et al. Increased central artery stiffness in impaired glucose metabolism and type 2 diabetes: the Hoorn Study. Hypertension. 2004;43:176-181.

15. Van Bortel LM, Duprez D, Starmans-Kool MJ, et al. Clinical applications of arterial stiffness, Task Force III: recommendations for user procedures. Am J Hypertens. 2002;15:445-452.

16. Weber T, Ammer M, Rammer M, et al. Noninvasive determination of carotid-femoral pulse wave velocity depends critically on assessment of travel distance: a comparison with invasive measurement. J Hypertens. 2009;27:1624-1630.

17. Wendel-Vos GC, Schuit AJ, Saris WH, Kromhout D. Reproducibility and relative validity of the short questionnaire to assess health-enhancing physical activity. J Clin Epidemiol. 2003;56:1163-1169.

18. Stehouwer CD, Henry RM, Ferreira I. Arterial stiffness in diabetes and the metabolic syndrome: a pathway to cardiovascular disease. Diabetologia. 2008;51:527-539.

19. Van Bortel LM, Balkestein EJ, van der Heijden-Spek JJ, et al. Non-invasive assessment of local arterial pulse pressure: comparison of applanation tonometry and echo-tracking. J Hypertens. 2001;19:10371044.

20. Maher E EM, Creane A, Lally C, Kelly DJ. Site specific inelasticity of arterial tissue. J Biomech. 2021;8:1393-1399.

21. Benetos A, Laurent S, Hoeks AP, Boutouyrie PH, Safar ME. Arterial alterations with aging and high blood pressure. A noninvasive study of carotid and femoral arteries. Arterioscler Thromb. 1993;13:90-97. 


\section{4 | Chapter 3}

22. Van Bortel LM, Struijker-Boudier HA, Safar ME. Pulse pressure, arterial stiffness, and drug treatment of hypertension. Hypertension. 2001;38:914-921.

23. Vlachopoulos C, Aznaouridis K, O'Rourke MF, Safar ME, Baou K, Stefanadis C. Prediction of cardiovascular events and all-cause mortality with central haemodynamics: a systematic review and meta-analysis. Eur Heart J. 2010;31:1865-1871.

24. Wang KL, Cheng HM, Sung SH, et al. Wave reflection and arterial stiffness in the prediction of 15-year all-cause and cardiovascular mortalities: a community-based study. Hypertension. 2010;55:799-805.

25. Chirinos JA, Kips JG, Jacobs DR, Jr., et al. Arterial Wave Reflections and Incident Cardiovascular Events and Heart Failure: MESA (Multiethnic Study of Atherosclerosis). J Am Coll Cardiol. 2012;60:2170-2177.

26. Safar ME, Balkau B, Lange $C$, et al. Hypertension and vascular dynamics in men and women with metabolic syndrome. J Am Coll Cardiol. 2013;61:12-19.

27. Pannier B, Guerin AP, Marchais SJ, Safar ME, London GM. Stiffness of capacitive and conduit arteries: prognostic significance for end-stage renal disease patients. Hypertension. 2005;45:592-596.

28. van Dijk RA, van Ittersum FJ, Westerhof N, van Dongen EM, Kamp O, Stehouwer CD. Determinants of brachial artery mean $24 \mathrm{~h}$ pulse pressure in individuals with Type II diabetes mellitus and untreated mild hypertension. Clin Sci (Lond). 2002;102:177-186. 


\section{Supplemental Material}

\section{Supplemental methods}

\section{Determination of local arterial stiffness indices}

A single observer unaware of the participants' clinical or glucose tolerance status obtained properties of the right common carotid (10 $\mathrm{mm}$ proximal to the carotid bulb), the right common femoral (20 mm proximal to the flow divider), and the right brachial $(20 \mathrm{~mm}$ proximal to the antecubital fossa) arteries, with the use of an ultrasound scanner equipped with an 7.5-MHz linear probe (350 Series, Pie Medical, Maastricht, the Netherlands). ${ }^{1}$ The scanner was connected to a PC equipped with vessel wall movement detection software and an acquisition system (Wall Track System, Pie Medical, Maastricht, the Netherlands). This setup enables measurements of diameter, distension, and intima-media thickness (IMT). ${ }^{2,3}$ The temporal resolution of this system was approximately 10 ms. Briefly, after a 15-minute supine rest, the artery was visualized in B-mode. An M-line was then placed at the measurement site. After switching to M-mode, data acquisition in a real-time A-mode presentation on the computer screen was enabled after trackball-assisted identification of the arterial lumen. Data were then obtained during 3 consecutive 4 seconds measurements, triggered by the R-top of a simultaneously recorded ECG. The first radiofrequency signal was displayed on the screen, enabling the observer to check if the markers, positioned by the Wall Track System, coincided with the anterior and posterior wall reflections in the diastolic phase of the cardiac cycle. The cumulative radiofrequency signals were then digitized and stored. The change in diameter as a function of time (distension) was estimated and presented on the computer screen (distension waveform). Diastolic diameter was calculated as the difference in position between the anterior and posterior wall markers. Additionally, the carotid posterior wall IMT was calculated as the distance from the leading edge interface between lumen and intima to the leading edge interface between media and adventitia. The mean diameter, distension, and IMT of the 3 measurements were used in the analyses.

Reproducibility was assessed in 10 individuals ( 5 men; $58.2 \pm 9.5$ years) who were examined twice, 2 weeks apart. The intraobserver intersession coefficients of variation (CV = [standard deviation of the mean difference /V2] / pooled mean) were as follows: carotid IMT: 10.9\%; diameters: $2.9 \%$ (carotid), $2.5 \%$ (femoral), and 4.3\% (brachial); distension: $5.3 \%$ (carotid), $11.6 \%$ (femoral), and $12.8 \%$ (brachial); DC: $7.0 \%$ (carotid), $11.3 \%$ (femoral), and $12.8 \%$ (brachial); CC: 6.3\% (carotid), 13.1\% (femoral), and 13.9\% (brachial); carotid YEM, 11.6\%; and aortic pressures: 5.2\% (systolic), 3.4\% (diastolic), 3.8\% (pulse), and 3.2\% (mean). 


\section{Determination of cfPWV, Aix and SAC}

Segmental aortic stiffness was determined by assessment of cfPWV (i.e. the ratio of travelled distance divided by transit time). The travel distance was estimated using body height according to the formula proposed by Weber et al. ${ }^{4}$ Carotid-femoral transit time was determined by continuous measurement of the distension curves of the carotid and femoral artery. ${ }^{5}$ To obtain carotid-femoral transit time, we assessed the average time delay (mean of 3 recordings of 4 seconds per artery) from the ECG trigger to $10 \%$ of the ascending slope of the distension curve of both arteries and subtracted the carotid value from the femoral value. The Aix was determined by radial applanation tonometry (Sphygmocor, Atcor Medical, Sydney, Australia). ${ }^{5,6}$ SAC was determined according to two methods: the exponential decay method based on the Windkessel method, and the ratio of stroke volume to aortic PP, as described previously. ${ }^{5}$ The first method used data obtained by applanation tonometry. The second method used the ratio of stroke volume to aortic PP to determine total arterial compliance. Stroke volume was calculated as cardiac output (determined by echocardiography ${ }^{7}$ ) divided by heart rate. Aortic PP was calculated by calibration. ${ }^{5}$

\section{References}

1. Henry RM, Kostense PJ, Spijkerman AM, et al. Arterial stiffness increases with deteriorating glucose tolerance status: the Hoorn Study. Circulation. 2003;107:2089-2095.

2. Hoeks AP, Brands PJ, Willigers JM, Reneman RS. Non-invasive measurement of mechanical properties of arteries in health and disease. Proc Inst Mech Eng H. 1999;213:195-202.

3. Hoeks AP, Willekes C, Boutouyrie P, Brands PJ, Willigers JM, Reneman RS. Automated detection of local artery wall thickness based on M-line signal processing. Ultrasound Med Biol. 1997;23:1017-1023.

4. Weber T, Ammer M, Rammer M, et al. Noninvasive determination of carotid-femoral pulse wave velocity depends critically on assessment of travel distance: a comparison with invasive measurement. J Hypertens. 2009;27:1624-1630.

5. Schram MT, Henry RM, van Dijk RA, et al. Increased central artery stiffness in impaired glucose metabolism and type 2 diabetes: the Hoorn Study. Hypertension. 2004;43:176-181.

6. Laurent S, Cockcroft J, Van Bortel L, et al. Expert consensus document on arterial stiffness: methodological issues and clinical applications. Eur Heart J. 2006;27:2588-2605.

7. Henry RM, Kamp O, Kostense PJ, et al. Left ventricular mass increases with deteriorating glucose tolerance, especially in women: independence of increased arterial stiffness or decreased flowmediated dilation: the Hoorn study. Diabetes Care. 2004;27:522-529.

\section{Supplemental results}

\section{Additional analysis femoral DC and CC}

The results were somewhat inconsistent for the femoral DC and CC (i.e. DC was significantly associated with CV events, but not with all-cause mortality, whereas CC was significantly associated with mortality, but not with CV events). To further explore these findings, we evaluated the association between, on the one hand, the individual elements of DC and CC (i.e. femoral diameter, distension and PP), and, on the other, CV events and all-cause mortality. The results showed that all individual elements contributed to the association with CV events and mortality (data not shown). Therefore, we believe that the discrepancy between the DC and CC may be the result of the play of chance, and cannot be explained 
by a particular remodelling pattern (e.g. a change primarily in diameter which impacts more on the $\mathrm{CC}$ ).

Table S3.1. Associations between arterial stiffness indices of the carotid, femoral and brachial artery with incident cardiovascular events and all-cause mortality; additional adjustments for carotid-femoral pulse wave velocity ${ }^{\mathrm{A}}$

\begin{tabular}{cccccc}
\hline \multirow{2}{*}{ Model } & \multicolumn{2}{c}{ Carotid artery } & \multicolumn{2}{c}{ Femoral artery } \\
\cline { 2 - 6 } & \multicolumn{2}{c}{ DC } & YEM & CC & DC \\
\hline \multicolumn{2}{l}{ Incident cardiovascular events } \\
1 & $1.10(0.78 ; 1.54)$ & $1.12(0.85 ; 1.49)$ & $0.99(0.74 ; 1.36)$ & $1.69(1.12 ; 2.46)$ & $1.43(1.00 ; 2.05)$ \\
2 & $1.00(0.69 ; 1.45)$ & $1.05(0.76 ; 1.46)$ & $0.97(0.71 ; 1.32)$ & $1.65(1.12 ; 2.42)$ & $1.39(0.96 ; 2.01)$ \\
3 & $0.95(0.64 ; 1.41)$ & $1.02(0.72 ; 1.45)$ & $1.03(0.75 ; 1.40)$ & $1.63(1.10 ; 2.41)$ & $1.45(0.98 ; 2.14)$ \\
4 & $0.89(0.61 ; 1.31)$ & $1.00(0.69 ; 1.46)$ & $1.03(0.75 ; 1.40)$ & $1.64(1.11 ; 2.42)$ & $1.47(0.98 ; 2.20)$ \\
All-cause mortality & & & & \\
1 & $1.84(1.16 ; 2.92)$ & $1.72(1.32 ; 2.24)$ & $1.75(1.12 ; 2.74)$ & $1.48(0.94 ; 2.34)$ & $1.30(0.85 ; 2.00)$ \\
2 & $1.76(1.04 ; 2.97)$ & $1.69(1.26 ; 2.25)$ & $1.64(1.05 ; 2.57)$ & $1.37(0.86 ; 2.20)$ & $1.20(0.77 ; 1.87)$ \\
3 & $1.70(1.01 ; 2.85)$ & $1.65(1.22 ; 2.22)$ & $1.64(1.07 ; 2.52)$ & $1.40(0.87 ; 2.24)$ & $1.08(0.69 ; 1.67)$ \\
4 & $1.67(1.00 ; 2.81)$ & $1.66(1.23 ; 2.27)$ & $1.63(1.06 ; 2.50)$ & $1.40(0.87 ; 2.24)$ & $1.07(0.68 ; 1.66)$ \\
\hline
\end{tabular}

Values are hazard ratio (95\% confidence interval). Hazard ratios are indicated per 1 SD lower distensibility (DC) and compliance coefficient (CC) and per 1 SD higher Young's elastic modulus (YEM). Model 1: adjusted for age, sex and glucose metabolism status; model 2: model 1 plus mean arterial pressure; model 3: model 2 plus prior cardiovascular disease, BMI, triglycerides, total/HDL cholesterol ratio, eGFR, (micro)albuminuria, physical activity, and smoking habits; model 4: model $3+$ cfPWV.

Number of events for incident cardiovascular events / all-mortality: 53 / 35.

${ }^{A}$ Analyses done in a subsample $(n=237)$ of the study population with available data on cfPWV. The effect estimates, therefore, differ from those obtained in the total study population. Participants with missing cfPWV data were, as compared to those without, older (71 years vs. 68 years), had a higher BMI $\left(27.7 \mathrm{~kg} / \mathrm{m}^{2} \mathrm{vs.} 25.6 \mathrm{~kg} / \mathrm{m}^{2}\right)$, a higher HbA1c $(6.1 \%$ vs. $5.9 \%)$, more often had hypertension $(73.5 \%$ vs. $62.1 \%)$, and had stiffer carotid arteries (distensibility coefficient $10.510^{-3} / \mathrm{kPa}$ vs. $11.410^{-3} / \mathrm{kPa}$ ); $\mathrm{P}$ for all <.05.

Table S3.2. Associations between arterial stiffness indices of carotid and femoral arteries with incident cardiovascular events and all-cause mortality; indices calculated with distension-waveform-calibrated pulse pressure instead of brachial pulse pressure

\begin{tabular}{cccccc}
\hline \multirow{2}{*}{ Model } & \multicolumn{3}{c}{ Carotid artery } & \multicolumn{2}{c}{ Femoral artery } \\
\cline { 2 - 6 } & \multicolumn{2}{c}{ DC } & YEM & CC & DC \\
\hline \multicolumn{2}{c}{ Incident cardiovascular events } & & & & \\
1 & $1.24(0.98 ; 1.58)$ & $1.24(1.04 ; 1.47)$ & $1.08(0.87 ; 1.35)$ & $1.38(1.07 ; 1.77)$ & $1.28(0.99 ; 1.65)$ \\
2 & $1.21(0.93 ; 1.57)$ & $1.23(1.02 ; 1.47)$ & $1.06(0.85 ; 1.32)$ & $1.35(1.05 ; 1.75)$ & $1.25(0.96 ; 1.62)$ \\
3 & $1.16(0.89 ; 1.51)$ & $1.17(0.97 ; 1.40)$ & $1.11(0.89 ; 1.38)$ & $1.37(1.06 ; 1.76)$ & $1.23(0.95 ; 1.59)$ \\
All-cause mortality & & & & \\
1 & $1.44(1.07 ; 1.95)$ & $1.30(1.09 ; 1.55)$ & $1.46(1.08 ; 1.97)$ & $1.51(1.08 ; 2.11)$ & $1.60(1.11 ; 2.31)$ \\
2 & $1.38(0.99 ; 1.93)$ & $1.27(1.04 ; 1.55)$ & $1.40(1.03 ; 1.90)$ & $1.45(1.03 ; 2.04)$ & $1.53(1.06 ; 2.23)$ \\
3 & $1.38(0.98 ; 1.94)$ & $1.27(1.03 ; 1.57)$ & $1.45(1.06 ; 1.97)$ & $1.46(1.03 ; 2.06)$ & $1.50(1.02 ; 2.20)$ \\
\hline
\end{tabular}

Values are hazard ratio (95\% confidence interval). Hazard ratios are indicated per 1 SD lower distensibility (DC) and compliance coefficient (CC) and per 1 SD higher Young's elastic modulus (YEM). Model 1: adjusted for age, sex and glucose metabolism status; model 2: model 1 plus mean arterial pressure; model 3: model 2 plus prior cardiovascular disease, BMI, triglycerides, total/HDL cholesterol ratio, eGFR, (micro)albuminuria, physical activity and smoking habits.

Number of participants and events available for analysis with incident cardiovascular events / all-cause mortality: for carotid DC, CC and YEM 473 (115 events) / 511 (79 events); and for femoral DC and CC 453 (110 events) / 490 (76 events). 
Table S3.3. Associations between arterial stiffness indices of the carotid and femoral artery and the aorta with incident cardiovascular events and all-cause mortality; additional adjustments for intima-media thickness, and systolic and pulse pressure instead of mean arterial pressure

\begin{tabular}{|c|c|c|c|c|c|c|}
\hline \multirow[t]{2}{*}{ Model } & \multicolumn{3}{|c|}{ Carotid artery } & \multicolumn{2}{|c|}{ Femoral artery } & \multirow{2}{*}{$\begin{array}{c}\text { Aorta } \\
\text { cfPWV }\end{array}$} \\
\hline & DC & YEM & $\mathrm{CC}$ & $\mathrm{DC}$ & $\mathrm{CC}$ & \\
\hline \multicolumn{7}{|l|}{ Incident cardiovascular events } \\
\hline 1: fully adjusted model & $\begin{array}{c}1.22 \\
(0.95 ; 1.56)\end{array}$ & $\begin{array}{c}1.19 \\
(1.00 ; 1.41)\end{array}$ & $\begin{array}{c}1.08 \\
(0.88 ; 1.31)\end{array}$ & $\begin{array}{c}1.39 \\
(1.06 ; 1.83)\end{array}$ & $\begin{array}{c}1.25 \\
(0.96 ; 1.63)\end{array}$ & $\begin{array}{c}1.56 \\
(1.23 ; 1.98)\end{array}$ \\
\hline 2: model $1+$ IMT & $\begin{array}{c}1.17 \\
(0.90 ; 1.50)\end{array}$ & $\begin{array}{c}1.25 \\
(1.05 ; 1.48)\end{array}$ & $\begin{array}{c}1.10 \\
(0.90 ; 1.35)\end{array}$ & $\begin{array}{c}1.37 \\
(1.04 ; 1.79)\end{array}$ & $\begin{array}{c}1.23 \\
(0.94 ; 1.61)\end{array}$ & $\begin{array}{c}1.56 \\
(1.23 ; 1.98)\end{array}$ \\
\hline $\begin{array}{l}\text { 3: model } 1+\text { SBP instead of } \\
\text { MAP }\end{array}$ & $\begin{array}{c}1.15 \\
(0.91 ; 1.45)\end{array}$ & $\begin{array}{c}1.16 \\
(0.97 ; 1.38)\end{array}$ & $\begin{array}{c}1.07 \\
(0.88 ; 1.30)\end{array}$ & $\begin{array}{c}1.32 \\
(1.01 ; 1.73)\end{array}$ & $\begin{array}{c}1.19 \\
(0.92 ; 1.55)\end{array}$ & $\begin{array}{c}1.50 \\
(1.17 ; 1.93)\end{array}$ \\
\hline $\begin{array}{l}\text { 4: model } 1+\text { PP instead of } \\
\text { MAP }\end{array}$ & $\begin{array}{c}1.15 \\
(0.92 ; 1.45)\end{array}$ & $\begin{array}{c}1.16 \\
(0.97 ; 1.37)\end{array}$ & $\begin{array}{c}1.07 \\
(0.89 ; 1.30)\end{array}$ & $\begin{array}{c}1.31 \\
(1.00 ; 1.72)\end{array}$ & $\begin{array}{c}1.18 \\
(0.91 ; 1.54)\end{array}$ & $\begin{array}{c}1.43 \\
(1.12 ; 1.83)\end{array}$ \\
\hline \multicolumn{7}{|l|}{ All-cause mortality } \\
\hline 1: fully adjusted model & $\begin{array}{c}1.51 \\
(1.11 ; 2.06)\end{array}$ & $\begin{array}{c}1.28 \\
(1.07 ; 1.53)\end{array}$ & $\begin{array}{c}1.43 \\
(1.10 ; 1.86)\end{array}$ & $\begin{array}{c}1.27 \\
(0.90 ; 1.79)\end{array}$ & $\begin{array}{c}1.47 \\
(1.01 ; 2.13)\end{array}$ & $\begin{array}{c}1.13 \\
(0.83 ; 1.54)\end{array}$ \\
\hline 2: model $1+$ IMT & $\begin{array}{c}1.52 \\
(1.13 ; 2.05)\end{array}$ & $\begin{array}{c}1.30 \\
(1.09 ; 1.55)\end{array}$ & $\begin{array}{c}1.45 \\
(1.10 ; 1.90)\end{array}$ & $\begin{array}{c}1.40 \\
(0.97 ; 2.00)\end{array}$ & $\begin{array}{c}1.51 \\
(1.03 ; 2.22)\end{array}$ & $\begin{array}{c}1.15 \\
(0.84 ; 1.57)\end{array}$ \\
\hline $\begin{array}{l}\text { 3: model } 1+\text { SBP instead of } \\
\text { MAP }\end{array}$ & $\begin{array}{c}1.58 \\
(1.18 ; 2.12)\end{array}$ & $\begin{array}{c}1.32 \\
(1.12 ; 1.56)\end{array}$ & $\begin{array}{c}1.48 \\
(1.14 ; 1.92)\end{array}$ & $\begin{array}{c}1.32 \\
(0.94 ; 1.85)\end{array}$ & $\begin{array}{c}1.52 \\
(1.05 ; 2.20)\end{array}$ & $\begin{array}{c}1.09 \\
(0.80 ; 1.48)\end{array}$ \\
\hline $\begin{array}{l}\text { 4: model } 1+\text { PP instead of } \\
\text { MAP }\end{array}$ & $\begin{array}{c}1.57 \\
(1.17 ; 2.10)\end{array}$ & $\begin{array}{c}1.33 \\
(1.13 ; 1.56)\end{array}$ & $\begin{array}{c}1.48 \\
(1.14 ; 1.92)\end{array}$ & $\begin{array}{c}1.29 \\
(0.93 ; 1.81)\end{array}$ & $\begin{array}{c}1.49 \\
(1.03 ; 2.15)\end{array}$ & $\begin{array}{c}1.07 \\
(0.78 ; 1.47)\end{array}$ \\
\hline
\end{tabular}

Values are hazard ratio (95\% confidence interval). Hazard ratios are indicated per 1 SD lower distensibility (DC) and compliance coefficient (CC) and per 1 SD higher Young's elastic modulus (YEM) and carotid-femoral pulse wave velocity (cfPWV). Model 1: adjusted for age, gender and glucose metabolism status, mean arterial pressure (MAP), heart rate, prior cardiovascular disease, BMI, triglycerides, total/HDL cholesterol ratio, eGFR, (micro)albuminuria, physical activity and smoking habits.

Number of participants and events available for analysis with incident cardiovascular events / all-cause mortality: for carotid DC, CC and YEM 533 (130 events) / 579 (96 events), for femoral DC and CC 461 (111 events) / 498 (77 events), and for cfPWV 215 (53 events) / 237 (36 events).

Table S3.4. Associations between arterial stiffness indices of carotid and femoral arteries and the aorta with incident cerebrovascular and coronary heart disease events

\begin{tabular}{|c|c|c|c|c|c|c|}
\hline \multirow[t]{2}{*}{ Model } & \multicolumn{3}{|c|}{ Carotid artery } & \multicolumn{2}{|c|}{ Femoral artery } & \multirow{2}{*}{$\begin{array}{l}\text { Aorta } \\
\text { cfPWV }\end{array}$} \\
\hline & $D C$ & YEM & CC & DC & CC & \\
\hline
\end{tabular}

Incident cerebrovascular events

$1 \quad 1.33(0.90 ; 1.95) 1.08(0.78 ; 1.49) 1.01(0.72 ; 1.41) 1.34(0.85 ; 2.13) 1.01(0.67 ; 1.54) 1.03(0.64 ; 1.68)$

$21.36(0.89 ; 2.08) 1.05(0.73 ; 1.50) 0.99(0.71 ; 1.40) 1.33(0.88 ; 2.01) 0.99(0.64 ; 1.52) 1.15(0.70 ; 1.89)$

Incident coronary heart disease events

$1 \quad 1.06(0.77 ; 1.45) 1.07(0.84 ; 1.37) 0.96(0.74 ; 1.26) 1.27(0.86 ; 1.87) 1.30(0.87 ; 1.94) 1.34(0.78 ; 2.29)$

$21.14(0.81 ; 1.62) 1.14(0.89 ; 1.47) \quad 0.99(0.75 ; 1.30) \quad 1.33(0.88 ; 2.01) 1.35(0.89 ; 2.04) 1.19(0.65 ; 2.19)$

Values are hazard ratio (95\% confidence interval). Hazard ratios are indicated per 1 SD lower distensibility (DC) and compliance coefficient (CC) and per 1 SD higher Young's elastic modulus (YEM) and carotid-femoral pulse wave velocity (cfPWV). Model 1: adjusted for age, sex and glucose metabolism status; model 2: model 1 plus mean arterial pressure.

Number of events for incident cerebrovascular events / coronary heart disease (CHD) events: for carotid DC, CC and YEM 40 / 57, for femoral DC and CC 33 / 49 and cfPWV 24 / 12. 
Table S3.5. Associations between elastance-thickness product of the carotid artery with incident cardiovascular events and all-cause mortality

\begin{tabular}{lc}
\hline Model & Eh \\
\hline Incident cardiovascular events & \\
1 & $1.23(1.06 ; 1.44)$ \\
2 & $1.22(1.04 ; 1.44)$ \\
3 & $1.21(1.02 ; 1.43)$ \\
All-cause mortality & \\
1 & $1.33(1.15 ; 1.54)$ \\
2 & $1.27(1.08 ; 1.49)$ \\
3 & $1.27(1.07 ; 1.50)$ \\
\hline
\end{tabular}

Values are hazard ratio (95\% confidence interval). Hazard ratios are indicated per $1 \mathrm{SD}$ higher elastance-wall thickness product (Eh). Model 1: adjusted for age, sex and glucose metabolism status; model 2: model 1 plus mean arterial pressure; model 3: model 2 plus prior cardiovascular disease, BMI, triglycerides, total/HDL

cholesterol ratio, eGFR, (micro)albuminuria, physical activity and smoking habits. Number of participants and events available for analysis with incident cardiovascular events / all-cause mortality 533 (130 events) / 579 (96 events). 


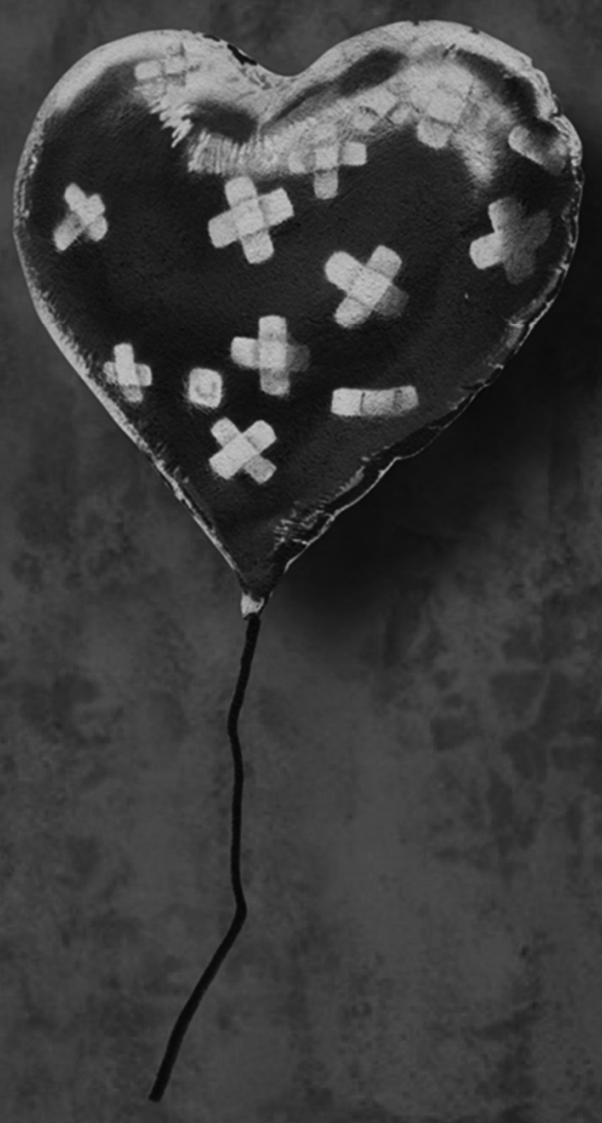

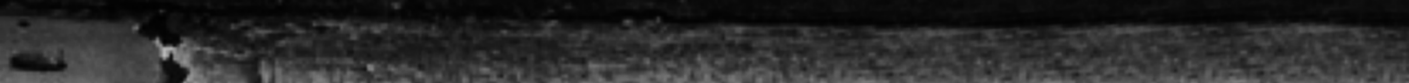
1. 1. 1. 


\section{Chapter 4}

\section{Carotid stiffness is associated with incident stroke: a systematic review and meta-analysis}

TT van Sloten*, S Sedaghat*, S Laurent, GM London, B Pannier, MA Ikram, M Kavousi, F Mattace-Raso, OH Franco, P Boutouyrie, CDA Stehouwer

*Both authors contributed equally 


\begin{abstract}

\section{Background}

Carotid stiffening is considered a key element in the pathogenesis of stroke. However, results of studies on the association between carotid stiffness and incident stroke have been inconsistent. We therefore investigated whether carotid stiffness (as determined by ultrasonography) is associated with incident stroke, and whether this association is independent of aortic stiffness as estimated by carotid-femoral pulse wave velocity (cfPWV). In addition, we evaluated the incremental value of carotid stiffness for stroke risk prediction beyond Framingham stroke risk score factors and cfPWV.
\end{abstract}

\title{
Methods
}

We performed a systematic review and meta-analyses of aggregate and individual participant data (IPD). We searched MEDLINE and EMBASE for prospective studies on carotid stiffness and incident cardiovascular events and/or mortality, published between inception and September 2014. For the IPD meta-analysis, we requested individual level data of all studies with available data on carotid stiffness and cfPWV. Main outcomes and measures were hazard ratios (HRs) and risk reclassification measures (integrative discrimination improvement [IDI] and continuous net reclassification improvement [NRI]) for incident stroke, CHD events, total cardiovascular events and mortality.

\section{Results}

Ten studies ( $n=22,472 ; 219,386$ person-years follow-up) were included in the aggregate data meta-analysis and four ( $n=4,540 ; 52,476$ person-years follow-up) in the IPD metaanalysis. The aggregate data meta-analysis showed that, after adjustment for cardiovascular risk factors, greater carotid stiffness (per SD) was associated with incident stroke (HR 1.18 [95\% confidence interval 1.05 to 1.33]). In addition, carotid stiffness was associated with incident total cardiovascular events (HR 1.16 [1.07 to 1.26] and cardiovascular (1.30 [1.15 to 1.46]) and all-cause mortality (1.22 [1.22 to 1.34]), but not with coronary heart disease events (1.03 [0.98 to 1.10]). The IPD meta-analysis showed that additional adjustment for cfPWV did not materially change these associations. Carotid stiffness improved stroke risk prediction beyond Framingham stroke risk score factors and cfPWV (IDI: 0.4\%-point [0.1 to 0.6\%-point]); continuous NRI: $18.6 \%$ [5.8 to $31.3 \%]$ )).

\section{Conclusion}

Carotid stiffness is associated with incident stroke independently of aortic stiffness and cardiovascular factors. In addition, carotid stiffness improves stroke risk prediction beyond Framingham stroke risk score factors and aortic stiffness. This supports the concept that carotid stiffening is important in the pathogenesis of stroke and constitutes a potential target for stroke prevention strategies. 


\section{Introduction}

Stroke is one of the leading causes of disability and death worldwide. ${ }^{1}$ The global burden of stroke has greatly increased in the last decades, and will continue to increase in the coming years. ${ }^{1,2}$ Therefore, effective prevention strategies need to be developed, which requires a better understanding of the risk factors for stroke. ${ }^{1}$

Aging and cardiovascular disease (CVD) risk factors lead to stiffening of the common carotid artery, $^{3}$ which can be quantified non-invasively by measurement of local distensibility. ${ }^{3,4}$ Stiffening of carotid arteries impairs their cushioning function and increases pressure and flow pulsatility, which transmits distally into the cerebral circulation and, thus, may increase the risk of stroke. ${ }^{5,6}$ In addition, carotid stiffening may lead to stroke through development of (rupture-prone) atherosclerotic carotid plaques. ${ }^{7}$ However, results of studies ${ }^{6,8-10}$ on the association between carotid stiffness and incident stroke have not been consistent. One study ${ }^{8}$ reported a statistically significant association between carotid stiffness and incident stroke, whereas other, smaller, studies ${ }^{6,9,10}$ did not.

We therefore performed a systematic review and aggregate data meta-analysis of cohort studies on the association between carotid stiffness and incident stroke. Because carotidfemoral pulse wave velocity (cfPWV), a measure of aortic stiffness, ${ }^{3}$ is the most often used arterial stiffness measurement and is associated with incident CVD, ${ }^{11,12}$ we additionally performed an individual participant data (IPD) meta-analysis with data from cohorts with measures of both carotid stiffness and cfPWV, and evaluated whether the association between carotid stiffness and stroke (if any) is independent of cfPWV. In addition, to evaluate whether carotid stiffness has any potential of being used as a risk predictor of stroke, we quantified the incremental value of carotid stiffness for stroke risk prediction beyond Framingham stroke risk score factors and cfPWV. Finally, we evaluated the association between carotid stiffness and other cardiovascular outcomes than stroke, including coronary heart disease (CHD) events, non-fatal and fatal cardiovascular events, and all-cause mortality. 


\section{Methods}

This review is reported in accordance with the preferred reporting items for systematic reviews and meta-analyses (PRISMA) statement (see supplemental material, Appendix A). ${ }^{13}$

\section{Evaluation procedure}

Two independent reviewers (TVS and SS) selected all relevant studies based on title and abstract, retrieved selected full texts, performed an eligibility assessment, extracted data and assessed risk of bias (described below). A third reviewer was available to solve any persisting disagreements.

\section{Data sources and search strategy}

We identified relevant studies through a search of Medline and Embase from inception to September 15, 2014, without any language restriction (search terms are provided as supplemental material, Appendix B). In addition, we identified studies by reviewing the reference lists of all relevant articles identified and by discussion with researchers to identify unpublished data.

\section{Eligibility criteria and study selection}

For the systematic review and aggregate data meta-analysis, we considered eligible all prospective cohort studies in humans (of any age) that investigated the association between, on the one hand, carotid stiffness and, on the other, (non-fatal and/or fatal) incident stroke, CHD events and/or total cardiovascular events, and/or all-cause mortality. We selected all studies that measured common carotid artery properties (diameter and distention) by ultrasound, together with brachial or local carotid pulse pressure (PP), and calculated carotid artery distensibility coefficient (DC), Young's elastic modulus (YEM), compliance coefficient (CC) or beta-stiffness index (SI). DC represents arterial stiffness (the lower DC, the greater the stiffness). ${ }^{3,4}$ The other indices are closely related to the DC: higher YEM represents greater stiffness of the arterial wall material; lower CC represents lower arterial buffering capacity; and higher SI represents greater stiffness and takes into account the non-linear relation between pressure and carotid artery diameter. , $^{3,4}$

\section{Data extraction}

We used a predesigned data abstraction form to extract information on the following items: study size, location, population characteristics, measures of arterial stiffness, follow-up duration, type and number of events, reported risk estimates, and variable(s) that were adjusted for in the analyses. In the case of multiple publications, ${ }^{6,14-16}$ we included the most up-to-date or comprehensive information. For the aggregate data meta-analysis, additional information for two studies ${ }^{17,18}$ was requested from corresponding authors; none provided the requested data. 


\section{Risk of bias assessment}

Risk of bias was evaluated with the Newcastle-Ottawa Scale (NOS) (NOS is provided as supplemental material, Appendix C). ${ }^{19}$ The NOS includes items on participant selection, validity of measurements, whether or not results were adjusted for age and blood pressure, plus duration and completeness of follow-up.

\section{Individual participant data meta-analysis}

For the IPD meta-analysis, we requested individual level data of all studies eligible for the aggregate data meta-analysis with available data on cfPWV. All eligible studies provided the requested data. Individual data from these studies were collected and harmonized for the statistical analysis using PASW statistics (version 21, IBM, Chicago, Illinois, USA).

\section{Outcome definitions}

In both the aggregate data and IPD meta-analysis, outcome definitions were used as reported in the originally published articles (see supplemental material, Table S4.1). Stroke included non-fatal and fatal cerebral infarction and intracerebral hemorrhage; CHD events included non-fatal and fatal acute myocardial infarction, angina pectoris, coronary artery bypass grafting, percutaneous coronary intervention and sudden death; total cardiovascular events included non-fatal and fatal CHD events, stroke, congestive heart failure and peripheral arterial disease; cardiovascular mortality included all fatal cardiovascular events (as defined above); and all-cause mortality included death from any cause.

\section{Data synthesis and analysis}

All analyses were performed with Cochrane Review Manager (version 5.2) and R statistical software (version 2.15).

\section{Aggregate data meta-analysis}

Results were pooled for the association between one standard deviation (SD) greater carotid stiffness and incident stroke. In addition, we evaluated the association of carotid stiffness with CHD events, total cardiovascular events, and cardiovascular and all-cause mortality. Results were included for lower DC or, if not available, higher YEM, lower CC or higher SI. For studies that reported results on carotid stiffness calculated with brachial as well as local PP, we included the results on carotid stiffness calculated with brachial PP in the main analysis, because these were available in the largest number of participants. In a sensitivity analysis, results were pooled for carotid stiffness calculated with local PP. All included studies calculated hazard ratios (HRs), except one study ${ }^{20}$ which calculated an odds ratio. We treated this odds ratio as a HR. Pooled HRs were calculated using the randomeffects inverse variance method. For each study, we included the fully adjusted value for the HR. Heterogeneity between studies was investigated with Higgins $I^{2}$ statistic. Several 
sensitivity analyses were done: analyses were repeated after exclusion of studies with a relatively high risk of bias (NOS score $<7$ ); analyses were repeated with studies which obtained carotid stiffness data by echotracking, which is considered the "gold standard" measurement technique to assess common carotid artery properties ${ }^{3,21,22}$; and results were pooled for each stiffness index separately (for lower DC, higher YEM, lower CC and higher $\mathrm{SI}$, respectively).

\section{Individual participant data meta-analysis}

Missing values on covariates were imputed using the expectation maximization method (single imputation) for each cohort separately. Percentage of missing values on covariates was minimal (total 2.0\%). We first used a two-stage analysis approach ${ }^{23}$ with estimates of association calculated separately within each study before pooling across studies by the random-effects inverse variance method. We used Cox proportional hazard models with one SD lower carotid DC as the determinant and incident stroke as the outcome. Additionally, we evaluated the association of carotid stiffness with CHD events, total cardiovascular events, and cardiovascular and all-cause mortality. The associations were first adjusted for the following potential confounders: age, sex, mean arterial pressure, heart rate, body mass index, total/high density lipoprotein cholesterol ratio, triglycerides, current smoking, diabetes, prior CVD, the use of anti-hypertensive and lipid-modifying medication (model 1); and additionally for cfPWV (model 2). We checked whether the associations of carotid stiffness with outcomes were linear by visual inspection of graphs of carotid stiffness quartiles against the corresponding HR and formal testing for nonlinearity using cubic restricted splines. ${ }^{24}$ The proportional hazards assumption was assessed by tests and visual inspection of graphs based on Schoenfeld residuals. ${ }^{24}$

We then evaluated whether carotid stiffness has any potential of being used as a risk predictor of stroke. We considered this a "proof of concept" analysis rather than an analysis of clinical relevance, because the study populations included in the IPD analysis had a high absolute baseline risk of stroke (see below). This greatly limits the possibility of identifying clinically relevant improvement of risk estimation. Therefore, we used the integrated discrimination improvement (IDI) and the continuous (category-free) net reclassification index (NRI) to quantify the incremental value of carotid DC for prediction of stroke risk beyond Framingham risk score factors and cfPWV. We used a one-stage approach. ${ }^{23}$ The IDI is a measure that reflects the average improvement, in percent point, in predicted probabilities summed across events and nonevents. ${ }^{25}$ The continuous NRI is a measure of reclassification that quantifies the sum of the percentages, for events and nonevents separately, of individuals in whom the directional change in predicted risk was consistent with observed events; values can range between $-200 \%$ and $+200 \%{ }^{25}$ These analyses were done in individuals without a prior CVD and limited to a time horizon of 10 years. We first fitted a Cox proportional hazards model to the data using the Kaplan-Meier estimate ${ }^{26}$ on 
the basis of cfPWV and the Framingham stroke risk score factors, ${ }^{27}$ i.e. age, sex, systolic blood pressure, total and high density lipoprotein cholesterol, current smoking, diabetes, the use of anti-hypertensive medication and left ventricular hypertrophy. We refer to this model as the "base model". This base model was then extended by carotid DC, and the base and extended model were compared using the IDI and continuous NRI. Additionally, we calculated the (change in) C-statistic, a measure of risk discrimination. ${ }^{24}$ Confidence intervals for the IDI, NRI and C-statistic were calculated by bootstrapping $(1,000$ repetitions). Finally, we evaluated the incremental value of carotid DC beyond Framingham cardiovascular risk score factors ${ }^{28}$ (i.e. age, sex, systolic blood pressure, total and high density lipoprotein cholesterol, current smoking, diabetes and the use of anti-hypertensive medication) and cfPWV for risk prediction of CHD events, total cardiovascular events, and cardiovascular and all-cause mortality.

\section{Results}

\section{Selection process and study characteristics}

Figure 4.1 shows the selection process of included studies. Of the ten studies $6,8-10,15,20,29-32$ included in the aggregate data meta-analysis, four ${ }^{6,8-10}$ evaluated stroke ( $n=17,662$ with 898 events), five $6,8-10,30$ CHD events ( $n=21,080$ with 2,113 events), ten ${ }^{6,8-10,15,20,29-32}$ any cardiovascular events ( $n=22,214$ individuals with 3,010 events), seven ${ }^{6,9,10,15,29,31,32}$ cardiovascular mortality ( $n=8,534$ with 806 events) and five $6,10,15,29,32$ all-cause mortality ( $n=5,991$ with 2,062 events). For the Rotterdam Study ${ }^{10}$ and the study of Blacher et al. ${ }^{15}$ the original investigators were able to provide an update of previously published results with unpublished data on a higher number of participants and longer follow-up duration. The updated results of the Rotterdam Study were based on 4,713 individuals and a median follow-up duration of 12.0 years (previously published results ${ }^{10}$ were based on $n=2,835$ and 4.1 years follow-up), and the updated results of Blacher et al. were based on 156 individuals and a median follow-up duration of 5.1 years (previously published results ${ }^{15}$ were based on $\mathrm{n}=110$ and 4.4 years follow-up). The studies included were conducted in the general population (five studies $6,8,10,30,32$ ), or in individuals with chronic kidney disease (four studies $^{15,20,29,31}$ ) or prior CVD (one study ${ }^{9}$ ). The follow-up duration ranged from 2.8 to 13.8 years (full study characteristics are provided in the supplemental material, Tables S4.1S4.3). Four studies (the study of Blacher et al. ${ }^{15}$, and the Rotterdam ${ }^{10}$, Hoorn $^{6}$ and Nephrotest ${ }^{29}$ Studies) had data available on cfPWV and were included in the IPD metaanalysis. Of these, two studies (Rotterdam and Hoorn Studies) had data available on incident stroke ( $n=4,075$ with 351 events) and all four had data available on total cardiovascular events ( $n=4,395$ with 763 events). 


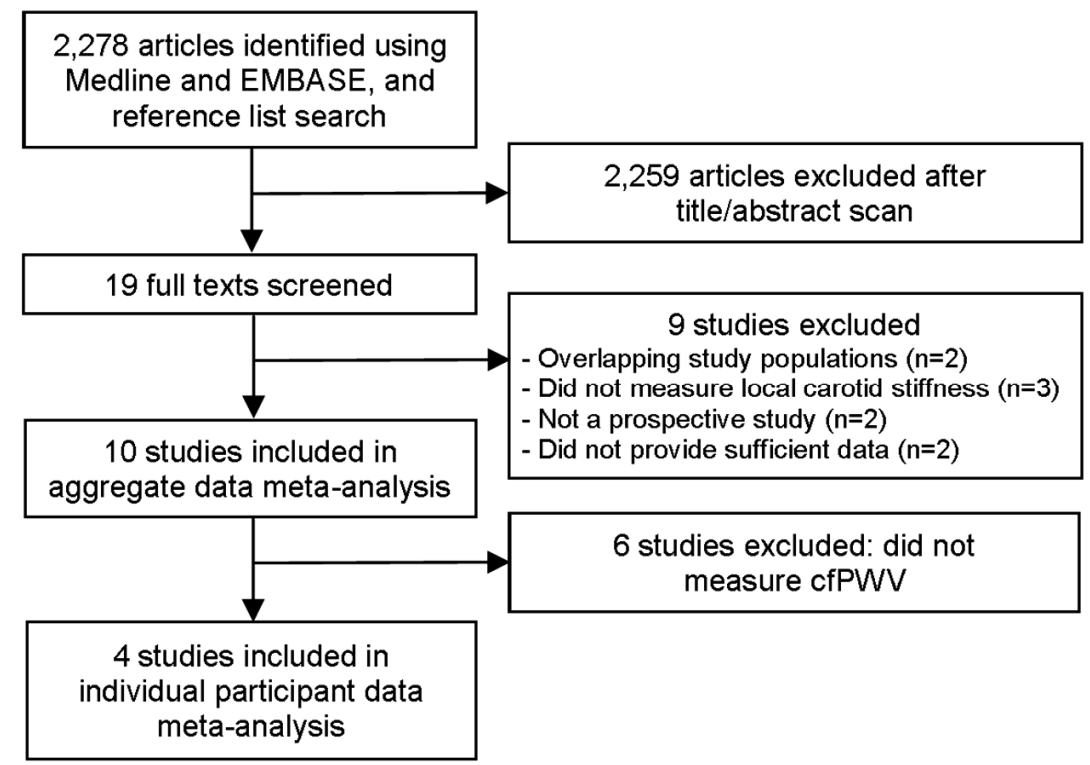

Figure 4.1. Flow chart of selection process of eligible studies.

\section{Risk of bias of individual studies}

Risk of bias among the included studies is presented in the supplemental material, Table S4.4. Overall, risk of bias was low (mean NOS score was 7 out of 8).

\section{Aggregate data meta-analysis}

Greater carotid stiffness was associated with a higher stroke incidence (Figure 4.2, Panel A). In addition, greater carotid stiffness was associated with a higher incidence of total cardiovascular events, and with greater cardiovascular and all-cause mortality, but not with CHD events (Figure 4.2, Panels B to E). The statistical heterogeneity between studies was low to moderate (range of $\mathrm{I}^{2}$ was $0 \%$ to $55 \%$; see also Figure 4.2 , Panels $A$ to E). Results did not materially change when data were pooled of carotid stiffness calculated with local PP; after exclusion of studies with a relatively high risk of bias; or when data were pooled of studies which obtained carotid stiffness data by echotracking (supplemental material, Figure S4.1). In addition, results were qualitatively similar for each carotid stiffness index, except for carotid CC, which was not statistically significantly associated with stroke or any of the other outcomes (Figure S4.1). 
A. Stroke

\section{Hazard ratio ( $95 \%$ confidence interval)}

$n$ for this analysis 17,662 with 898 events and 208,301 person-years of follow-up

ARIC Study

Hoorn Study

Rotterdam Study

SMART Study

$1^{2}=17 \%$

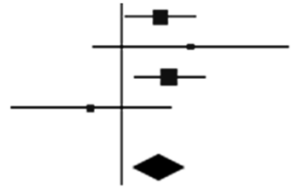

$1.19(1.02 ; 1.39)$

$1.36(0.89 ; 2.08)$

$1.24(1.07 ; 1.44)$

$0.88(0.62 ; 1.24)$

$1.18(1.05 ; 1.33)$

B. Coronary heart disease events

$n=21,080$ with 2,113 events; follow-up: 211,830 pers on-years

3City Study

ARIC Study

Hoorn Study

Rotterdam Study

SMART Study

$1^{2}=0 \%$

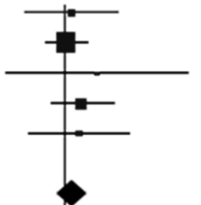

$1.03(0.86 ; 1.23)$

$1.01(0.94 ; 1.09)$

$1.14(0.81 ; 1.62)$

$1.07(0.95 ; 1.21)$

$1.06(0.87 ; 1.29)$

$1.03(0.98 ; 1.10)$

C. Total cardiovascular events

$n=22,214$ with 3,010 events: follow-up: 216,959 person-years

3City Study

ARIC Study

Barenbrock, 2002

Blacher, 2001

Hoorn Study

Nephrotest Study

Rotterdam Study

Shoji, 2010

SMART Study

Stork, 2004

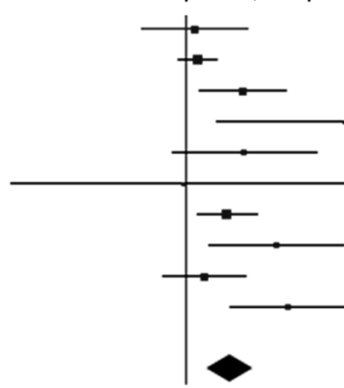

$1.03(0.86 ; 1.23)$

$1.04(0.97 ; 1.11)$

$1.21(1.05 ; 1.40)$

$1.72(1.11 ; 2.65)$

$1.22(0.95 ; 1.56)$

$0.99(0.55 ; 1.79)$

$1.15(1.04 ; 1.27)$

$1.36(1.08 ; 1.71)$

$1.06(0.92 ; 1.23)$

$1.42(1.17 ; 1.73)$

$1^{2}=55 \%$

$1.16(1.07 ; 1.26)$

D. Cardiovascular mortality

$n=8,534$ with 806 events; follow-up: 73,768 person-years

Blacher, 2001

Hoorn Study

Nephrotest Study

Rotterdam Study

Shoji, 2010

SMART Study

Stork, 2004

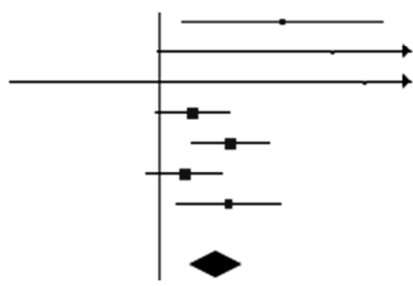

$1.72(1,11 ; 2.65)$

$2.14(0.99 ; 4.61)$

$2.47(0.53 ; 11.60)$

$1.16(0.99 ; 1.35)$

$1.36(1.08 ; 1.71)$

$1.12(0.95 ; 1.32)$

$1.42(1.17 ; 1.73)$

$1^{2}=38 \%$

$1.30(1.15 ; 1.46)$

E. All-cause mortality

$n=5,991$ with 2,062 events; follow-up: 65,771 person-years

Blacher, 2001

Hoorn Study

Nephrotest Study

Rotterdam Study

Stork, 2004

$P^{2}=15 \%$

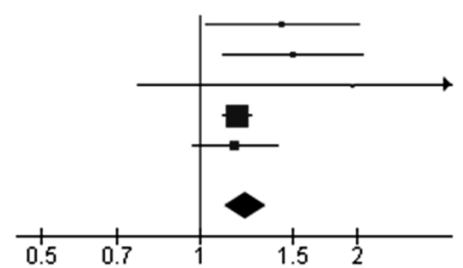

$1.44(1,03 ; 2.01)$

$1.51(1.11 ; 2.06)$

$1.95(0.76 ; 4.97)$

$1.18(1.10 ; 1.26)$

$1.17(0.97 ; 1.40)$

$1.22(1.12 ; 1.34)$

Figure 4.2. Results of aggregate data meta-analysis. Forest plot for the association between, on the one hand, one standard deviation (SD) greater carotid stiffness and, on the other, incident stroke (A), coronary heart disease events (B), total cardiovascular events (C), cardiovascular mortality (D) and all-cause mortality (E). For each study, the hazard ratio was pooled for, if available, one SD lower carotid distensibility coefficient; if not available, the hazard ratio was pooled for one SD higher Young's elastic modulus (for SMART Study; 3City Study; and Stork, 2004), or one SD higher beta-stiffness index (for Shoji, 2010).

ARIC = atherosclerosis risk in communities; SMART = second manifestations of arterial disease. 


\section{Discussion}

The present systematic review and meta-analysis of aggregate and individual participant data showed that greater carotid stiffness was associated with a higher stroke incidence. This association was independent of age, sex, blood pressure and CVD risk factors, and did not materially change after adjustment for cfPWV, a measure of aortic stiffness. In addition, estimation of carotid stiffness modestly improved stroke risk prediction beyond Framingham stroke risk score factors and cfPWV, as indicated by a statistically significant improvement of the IDI and continuous NRI. Finally, carotid stiffness was associated with a higher incidence of total cardiovascular events, and greater cardiovascular and all-cause mortality, but not with CHD events.

This is the first systematic review and meta-analysis on the association between carotid stiffness and incident cardiovascular disease and mortality. The findings are in agreement with, and further extend, previous observational studies ${ }^{6,8,20,29}$ that reported an association between carotid stiffness and incident CVD, 6,8,20,29 including stroke. ${ }^{8}$ The aggregate data meta-analysis enabled us to examine these associations in greater detail with enhanced power. In addition, the IPD meta-analysis allowed us to do a comprehensive range of additional analyses, including adjustment for cfPWV and quantification of stroke risk improvement beyond Framingham risk score factors and cfPWV.

Some methodological issues warrant consideration. First, the results were consistent across different study populations notwithstanding differences in methods to quantify carotid stiffness, and were not related to the risk of bias of included studies, which strengthens the validity of the findings. Second, the results were consistent for all carotid stiffness indices, except for carotid CC, which was not statistically significantly associated with stroke. To further explore this finding, we evaluated the association between individual elements of the stiffness indices (diameter, distension and PP) and stroke. The results showed that greater carotid diameter, lower distension and higher PP were each associated with a higher stroke incidence (data not shown). The association between greater carotid diameter and incident stroke is in accordance with previous studies, ${ }^{33,34}$ and may reflect arterial remodeling in response to atherosclerosis or increased arterial stiffness. ${ }^{14}$ However, arterial diameter enlargement leads to greater compliance, and this may explain that we did not find an association between (lower) carotid CC and stroke. Third, the present study had insufficient power to formally test the potential influence of publication bias. Nevertheless, a broad systematic search was done to identify all relevant studies, and we were able to include published as well as unpublished data. This limits the possibility of the presence of (substantial) publication bias. 
The present study showed that greater carotid stiffness is associated with a higher stroke incidence, independently of aortic stiffness, and supports the concept that carotid stiffening is important in the pathogenesis of stroke. ${ }^{6}$ The underlying mechanism may be that stiffening of the carotid artery (or of other elastic arteries for which the carotid artery may serve as a proxy) leads to a higher pulsatile pressure and flow load on the brain. ${ }^{3,4,35}$ This increased load can penetrate distally into the cerebral microcirculation and may directly cause cerebral ischemia and hemorrhage. 5,35,36 In addition, the increased pulsatile load may induce a hypertrophic remodeling response and rarefaction of small cerebral arteries, which, in turn, may lead to chronic ischemia. Furthermore, stiffening of the carotid artery may lead to stroke through local development of rupture-prone atherosclerotic plaques. Indeed, previous studies ${ }^{7,37}$ have shown that arterial stiffness is associated with presence ${ }^{7,37}$ and a rupture-prone phenotype ${ }^{7}$ (e.g. intraplaque hemorrhage) of atherosclerotic plaques in the internal carotid artery.

In the present study, carotid stiffness, in contrast to aortic stiffness (as determined by (fPWV) ${ }^{11,12}$, was not associated with incident CHD events. A possible explanation for these observations may be that stiffening of the aorta, but not of the carotid artery, leads to a higher left ventricular load and reduced diastolic coronary perfusion. . $^{3,4}$

In addition, carotid stiffness was associated with total (non-fatal and fatal) cardiovascular events and with all-cause mortality. This suggests that stiffening of carotid arteries additionally increases the risk of diseases other than stroke. For example, it is conceivable that stiffening of the carotid artery is associated with risk of congestive heart failure, as stiffening of the carotid artery could act as a proxy for stiffening of the proximal elastic segment of the aorta, which increases cardiac afterload and is associated with risk of congestive heart failure. ${ }^{38,39}$ In addition, carotid stiffness may be a marker of biological aging and, thus, be associated with mortality of age-related diseases other than cardiovascular disease. ${ }^{6}$ These possibilities require further investigation.

From a clinical point of view, the observation that carotid stiffness was associated with incident stroke independently of aortic stiffness is important, as this, together with experimental data, identifies carotid stiffness as a potential separate target for stroke risk lowering therapy. CVD risk factors have different impacts on stiffening of elastic versus muscular arteries. ${ }^{40,41}$ This may be attributed to the marked differences in the architecture of these arteries, and suggests that stiffness of elastic arteries may be specifically targeted. Currently, no therapy is available that specifically targets stiffness of elastic arteries.

In the present study, carotid stiffness improved risk prediction of stroke beyond Framingham stroke risk score factors and cfPWV, as indicated by improvement of IDI and continuous NRI. This finding provides proof of principle that carotid stiffness can have 
additional value as a risk predictor of stroke. The improvement of stroke risk prediction by carotid stiffness was, however, modest, and, in high-risk populations such as those included in the current analyses, such an improvement may not be clinically relevant. ${ }^{42}$ Nevertheless, the current data provide a framework for investigating whether assessment of carotid stiffness can improve stroke risk prediction in younger individuals and in those at intermediate risk, in whom improvement of risk prediction may be of greater importance. ${ }^{43}$

A limitation of the present study is that (unavoidable) survival bias may have led to an underestimation of the associations observed. In addition, we did not evaluate the association between carotid stiffness and stroke subtypes, i.e. ischemic versus hemorrhagic. However, it is likely that stiffening of the carotid artery increases the risk of both ischemic and hemorrhagic stroke. . $, 35,36$

In conclusion, the present study shows that greater carotid stiffness is associated with a higher stroke incidence independently of cfPWV. In addition, carotid stiffness modestly improved risk prediction of stroke beyond Framingham stroke risk score factors and cfPWV. This identifies carotid stiffness as a potential separate target for prevention strategies of stroke. Further studies are needed to quantify the predictive value of carotid stiffness in individuals at intermediate risk in whom reclassification improvement may be of greatest clinical importance. 


\section{References}

1. Feigin VL, Forouzanfar MH, Krishnamurthi R, et al. Global and regional burden of stroke during 19902010: findings from the Global Burden of Disease Study 2010. Lancet. 2014;383:245-254.

2. Ovbiagele B, Goldstein LB, Higashida RT, et al. Forecasting the future of stroke in the United States: a policy statement from the American Heart Association and American Stroke Association. Stroke. 2013;44:2361-2375.

3. Laurent S, Cockcroft J, Van Bortel L, et al. Expert consensus document on arterial stiffness: methodological issues and clinical applications. Eur Heart J. 2006;27:2588-2605.

4. Safar ME, O'Rourke MF. Arterial stiffness in hypertension. Amsterdam: Elsevier; 2006.

5. Mitchell GF. Effects of central arterial aging on the structure and function of the peripheral vasculature: implications for end-organ damage. J Appl Physiol (1985). 2008;105:1652-1660.

6. van Sloten TT, Schram MT, van den Hurk K, et al. Local stiffness of the carotid and femoral artery is associated with incident cardiovascular events and all-cause mortality: the Hoorn study. J Am Coll Cardiol. 2014;63:1739-1747.

7. Selwaness $M$, van den Bouwhuijsen $Q$, Mattace-Raso FU, et al. Arterial stiffness is associated with carotid intraplaque hemorrhage in the general population: the Rotterdam study. Arterioscler Thromb Vasc Biol. 2014;34:927-932.

8. Yang EY, Chambless L, Sharrett AR, et al. Carotid arterial wall characteristics are associated with incident ischemic stroke but not coronary heart disease in the Atherosclerosis Risk in Communities (ARIC) study. Stroke. 2012;43:103-108.

9. Dijk JM, Algra A, van der Graaf Y, Grobbee DE, Bots ML. Carotid stiffness and the risk of new vascular events in patients with manifest cardiovascular disease. The SMART study. Eur Heart J. 2005;26:12131220.

10. Mattace-Raso FU, van der Cammen TJ, Hofman A, et al. Arterial stiffness and risk of coronary heart disease and stroke: the Rotterdam Study. Circulation. 2006;113:657-663.

11. Ben-Shlomo Y, Spears M, Boustred C, et al. Aortic pulse wave velocity improves cardiovascular event prediction: an individual participant meta-analysis of prospective observational data from 17,635 subjects. J Am Coll Cardiol. 2014;63:636-646.

12. Vlachopoulos C, Aznaouridis K, Stefanadis C. Prediction of cardiovascular events and all-cause mortality with arterial stiffness: a systematic review and meta-analysis. J Am Coll Cardiol. 2010;55:1318-1327.

13. Moher D, Liberati A, Tetzlaff J, Altman DG, Group P. Preferred reporting items for systematic reviews and meta-analyses: the PRISMA statement. BMJ. 2009;339:b2535.

14. van Dijk RA, Dekker JM, Nijpels G, Heine RJ, Bouter LM, Stehouwer CD. Brachial artery pulse pressure and common carotid artery diameter: mutually independent associations with mortality in subjects with a recent history of impaired glucose tolerance. Eur J Clin Invest. 2001;31:756-763.

15. Blacher J, Guerin AP, Pannier B, Marchais SJ, London GM. Arterial calcifications, arterial stiffness, and cardiovascular risk in end-stage renal disease. Hypertension. 2001;38:938-942.

16. Blacher J, Pannier B, Guerin AP, Marchais SJ, Safar ME, London GM. Carotid arterial stiffness as a predictor of cardiovascular and all-cause mortality in end-stage renal disease. Hypertension. 1998;32:570-574.

17. Duprez d, Jacobs DR, Lutsey PL Bluemke DA, Brumback LC, Polak J, Kronmal RA. Small artery elasticity, but neither carotid artery elasticity nor aorta distensibility, predicts cardiovascular events in an asymptomatic - results of the multi-ethnic study of atherosclerosis. J Am Coll Cardiol. 2010:A133;E1252.

18. Haluska BA, Jeffries L, Carlier S, Marwick TH. Measurement of arterial distensibility and compliance to assess prognosis. Atherosclerosis. 2010;209:474-480.

19. Wells GA SB, O'Connell D, Peterson J, et al. The Newcastle-Ottawa Scale (NOS) for assessing the quality if nonrandomized studies in meta-analyses. www.ohri.ca/programs/clinical_epidemiology/oxford.htm. 
20. Barenbrock M, Kosch M, Joster E, Kisters K, Rahn KH, Hausberg M. Reduced arterial distensibility is a predictor of cardiovascular disease in patients after renal transplantation. J Hypertens. 2002;20:79-84.

21. Boutouyrie P, Bussy C, Lacolley P, Girerd X, Laloux B, Laurent S. Association between local pulse pressure, mean blood pressure, and large-artery remodeling. Circulation. 1999;100:1387-1393.

22. Laurent $\mathrm{S}$, Caviezel B, Beck L, et al. Carotid artery distensibility and distending pressure in hypertensive humans. Hypertension. 1994;23:878-883.

23. Pennells L, Kaptoge S, White IR, Thompson SG, Wood AM, Emerging Risk Factors C. Assessing risk prediction models using individual participant data from multiple studies. Am J Epidemiol. 2014;179:621-632.

24. Harrell FEJ. Regression Modeling Strategies: With Application to Linear Models, Logistic Regression, and Survival Analysis. New York: Springer Publishing; 2001.

25. Pencina MJ, D'Agostino RB, Vasan RS. Statistical methods for assessment of added usefulness of new biomarkers. Clin Chem Lab Med. 2010;48:1703-1711.

26. Steyerberg EW, Pencina MJ. Reclassification calculations for persons with incomplete follow-up. Ann Intern Med. 2010;152:195-196; author reply 196-197.

27. Wolf PA, D'Agostino RB, Belanger AJ, Kannel WB. Probability of stroke: a risk profile from the Framingham Study. Stroke. 1991;22:312-318.

28. D'Agostino RB, Sr., Vasan RS, Pencina MJ, et al. General cardiovascular risk profile for use in primary care: the Framingham Heart Study. Circulation. 2008;117:743-753.

29. Karras A, Haymann JP, Bozec E, et al. Large artery stiffening and remodeling are independently associated with all-cause mortality and cardiovascular events in chronic kidney disease. Hypertension. 2012;60:1451-1457.

30. Leone N, Ducimetiere P, Gariepy J, et al. Distension of the carotid artery and risk of coronary events: the three-city study. Arterioscler Thromb Vasc Biol. 2008;28:1392-1397.

31. Shoji T, Maekawa K, Emoto M, et al. Arterial stiffness predicts cardiovascular death independent of arterial thickness in a cohort of hemodialysis patients. Atherosclerosis. 2010;210:145-149.

32. Stork S, van den Beld AW, von Schacky C, et al. Carotid artery plaque burden, stiffness, and mortality risk in elderly men: a prospective, population-based cohort study. Circulation. 2004;110:344-348.

33. Baldassarre D, Hamsten A, Veglia F, et al. Measurements of carotid intima-media thickness and of interadventitia common carotid diameter improve prediction of cardiovascular events: results of the IMPROVE (Carotid Intima Media Thickness [IMT] and IMT-Progression as Predictors of Vascular Events in a High Risk European Population) study. J Am Coll Cardiol. 2012;60:1489-1499.

34. Polak JF, Sacco RL, Post WS, Vaidya D, Arnan MK, O'Leary DH. Incident stroke is associated with common carotid artery diameter and not common carotid artery intima-media thickness. Stroke. 2014;45:14421446.

35. Tzourio C, Laurent S, Debette S. Is hypertension associated with an accelerated aging of the brain? Hypertension. 2014;63:894-903.

36. Brisset M, Boutouyrie P, Pico F, et al. Large-vessel correlates of cerebral small-vessel disease. Neurology. 2013;80:662-669.

37. Zureik M, Temmar M, Adamopoulos C, et al. Carotid plaques, but not common carotid intima-media thickness, are independently associated with aortic stiffness. J Hypertens. 2002;20:85-93.

38. Coutinho T, Borlaug BA, Pellikka PA, Turner ST, Kullo IJ. Sex differences in arterial stiffness and ventricular-arterial interactions. J Am Coll Cardiol. 2013;61:96-103.

39. Tartiere-Kesri L, Tartiere JM, Logeart D, Beauvais F, Cohen Solal A. Increased proximal arterial stiffness and cardiac response with moderate exercise in patients with heart failure and preserved ejection fraction. J Am Coll Cardiol. 2012;59:455-461.

40. Paini A, Boutouyrie P, Calvet D, Tropeano Al, Laloux B, Laurent S. Carotid and aortic stiffness: determinants of discrepancies. Hypertension. 2006;47:371-376.

41. Stehouwer CD, Henry RM, Ferreira I. Arterial stiffness in diabetes and the metabolic syndrome: a pathway to cardiovascular disease. Diabetologia. 2008;51:527-539. 
76 | Chapter 4

42. Pencina MJ, D'Agostino RB, Pencina KM, Janssens AC, Greenland P. Interpreting incremental value of markers added to risk prediction models. Am J Epidemiol. 2012;176:473-481.

43. Lloyd-Jones DM. Cardiovascular risk prediction: basic concepts, current status, and future directions. Circulation. 2010;121:1768-1777. 


\section{Supplemental Material}

\section{Supplemental methods section}

\section{Appendix A: PRISMA 2009 checklist}

\begin{tabular}{|c|c|c|c|}
\hline Section/topic & & Checklist item & $\begin{array}{l}\text { Reported on } \\
\text { page: }\end{array}$ \\
\hline \multicolumn{4}{|l|}{ TITLE } \\
\hline Title & 1 & $\begin{array}{l}\text { Identify the report as a systematic review, meta-analysis, or } \\
\text { both. }\end{array}$ & 61 \\
\hline \multicolumn{4}{|l|}{ ABSTRACT } \\
\hline $\begin{array}{l}\text { Structured } \\
\text { summary }\end{array}$ & 2 & $\begin{array}{l}\text { Provide a structured summary including, as applicable: } \\
\text { background; objectives; data sources; study eligibility criteria, } \\
\text { participants, and interventions; study appraisal and synthesis } \\
\text { methods; results; limitations; conclusions and implications of key } \\
\text { findings; systematic review registration number. }\end{array}$ & 62 \\
\hline \multicolumn{4}{|l|}{ INTRODUCTION } \\
\hline \begin{tabular}{|l|} 
Rationale \\
\end{tabular} & 3 & $\begin{array}{l}\text { Describe the rationale for the review in the context of what is } \\
\text { already known. }\end{array}$ & 63 \\
\hline Objectives & 4 & $\begin{array}{l}\text { Provide an explicit statement of questions being addressed with } \\
\text { reference to participants, interventions, comparisons, outcomes, } \\
\text { and study design (PICOS). }\end{array}$ & 63 \\
\hline \multicolumn{4}{|l|}{ METHODS } \\
\hline $\begin{array}{l}\text { Protocol and } \\
\text { registration }\end{array}$ & 5 & $\begin{array}{l}\text { Indicate if a review protocol exists, if and where it can be } \\
\text { accessed (e.g., Web address), and, if available, provide } \\
\text { registration information including registration number. }\end{array}$ & - \\
\hline $\begin{array}{l}\text { Eligibility } \\
\text { criteria }\end{array}$ & 6 & $\begin{array}{l}\text { Specify study characteristics (e.g., PICOS, length of follow-up) } \\
\text { and report characteristics (e.g., years considered, language, } \\
\text { publication status) used as criteria for eligibility, giving rationale. }\end{array}$ & 64 \\
\hline $\begin{array}{l}\text { Information } \\
\text { sources }\end{array}$ & 7 & $\begin{array}{l}\text { Describe all information sources (e.g., databases with dates of } \\
\text { coverage, contact with study authors to identify additional } \\
\text { studies) in the search and date last searched. }\end{array}$ & 64 \\
\hline Search & 8 & $\begin{array}{l}\text { Present full electronic search strategy for at least one database, } \\
\text { including any limits used, such that it could be repeated. }\end{array}$ & $\begin{array}{l}\text { Supplemental } \\
\text { Material }\end{array}$ \\
\hline $\begin{array}{l}\text { Study } \\
\text { selection }\end{array}$ & 9 & $\begin{array}{l}\text { State the process for selecting studies (i.e., screening, eligibility, } \\
\text { included in systematic review, and, if applicable, included in the } \\
\text { meta-analysis). }\end{array}$ & 64 \\
\hline $\begin{array}{l}\text { Data collection } \\
\text { process }\end{array}$ & 10 & $\begin{array}{l}\text { Describe method of data extraction from reports (e.g., piloted } \\
\text { forms, independently, in duplicate) and any processes for } \\
\text { obtaining and confirming data from investigators. }\end{array}$ & 64 \\
\hline Data items & 11 & $\begin{array}{l}\text { List and define all variables for which data were sought (e.g., } \\
\text { PICOS, funding sources) and any assumptions and simplifications } \\
\text { made. }\end{array}$ & 64 \\
\hline $\begin{array}{l}\text { Risk of bias in } \\
\text { individual } \\
\text { studies }\end{array}$ & 12 & $\begin{array}{l}\text { Describe methods used for assessing risk of bias of individual } \\
\text { studies (including specification of whether this was done at the } \\
\text { study or outcome level), and how this information is to be used } \\
\text { in any data synthesis. }\end{array}$ & $\begin{array}{l}65, \\
\text { Supplemental } \\
\text { Material }\end{array}$ \\
\hline
\end{tabular}




\begin{tabular}{|c|c|c|c|}
\hline $\begin{array}{l}\text { Summary } \\
\text { measures }\end{array}$ & 13 & $\begin{array}{l}\text { State the principal summary measures (e.g., risk ratio, difference } \\
\text { in means). }\end{array}$ & $65-66$ \\
\hline $\begin{array}{l}\text { Synthesis of } \\
\text { results }\end{array}$ & 14 & $\begin{array}{l}\text { Describe the methods of handling data and combining results of } \\
\left.\text { studies, if done, including measures of consistency (e.g., }\left.\right|^{2}\right) \text { for } \\
\text { each meta-analysis. }\end{array}$ & $65-66$ \\
\hline $\begin{array}{l}\text { Risk of bias } \\
\text { across studies }\end{array}$ & 15 & $\begin{array}{l}\text { Specify any assessment of risk of bias that may affect the } \\
\text { cumulative evidence (e.g., publication bias, selective reporting } \\
\text { within studies). }\end{array}$ & $65-66$ \\
\hline $\begin{array}{l}\text { Additional } \\
\text { analyses }\end{array}$ & 16 & $\begin{array}{l}\text { Describe methods of additional analyses (e.g., sensitivity or } \\
\text { subgroup analyses, meta-regression), if done, indicating which } \\
\text { were pre-specified. }\end{array}$ & $65-66$ \\
\hline \multicolumn{4}{|l|}{ RESULTS } \\
\hline $\begin{array}{l}\text { Study } \\
\text { selection }\end{array}$ & 17 & $\begin{array}{l}\text { Give numbers of studies screened, assessed for eligibility, and } \\
\text { included in the review, with reasons for exclusions at each stage, } \\
\text { ideally with a flow diagram. }\end{array}$ & Figure 4.1 \\
\hline $\begin{array}{l}\text { Study } \\
\text { characteristic }\end{array}$ & 18 & $\begin{array}{l}\text { For each study, present characteristics for which data were } \\
\text { extracted (e.g., study size, PICOS, follow-up period) and provide } \\
\text { the citations. }\end{array}$ & $\begin{array}{l}\text { 67, Tables } \\
\text { S4.1-S4.3 }\end{array}$ \\
\hline $\begin{array}{l}\text { Risk of bias } \\
\text { within studies }\end{array}$ & 19 & $\begin{array}{l}\text { Present data on risk of bias of each study and, if available, any } \\
\text { outcome level assessment (see item 12). }\end{array}$ & $\begin{array}{l}68, \\
\text { Table S4.4 }\end{array}$ \\
\hline $\begin{array}{l}\text { Results of } \\
\text { individual } \\
\text { studies }\end{array}$ & 20 & $\begin{array}{l}\text { For all outcomes considered (benefits or harms), present, for } \\
\text { each study: (a) simple summary data for each intervention } \\
\text { group (b) effect estimates and confidence intervals, ideally with } \\
\text { a forest plot. }\end{array}$ & Figure 4.2 \\
\hline $\begin{array}{l}\text { Synthesis of } \\
\text { results }\end{array}$ & 21 & $\begin{array}{l}\text { Present results of each meta-analysis done, including confidence } \\
\text { intervals and measures of consistency. }\end{array}$ & 68 , Figure 4.2 \\
\hline $\begin{array}{l}\text { Risk of bias } \\
\text { across studies }\end{array}$ & 22 & $\begin{array}{l}\text { Present results of any assessment of risk of bias across studies } \\
\text { (see Item 15). }\end{array}$ & \begin{tabular}{|l|}
68, \\
Figure S4.1
\end{tabular} \\
\hline $\begin{array}{l}\text { Additional } \\
\text { analysis }\end{array}$ & 23 & $\begin{array}{l}\text { Give results of additional analyses, if done (e.g., sensitivity or } \\
\text { subgroup analyses, meta-regression [see Item 16]). }\end{array}$ & \begin{tabular}{|l|}
68,71 \\
Figure S4.1
\end{tabular} \\
\hline \multicolumn{4}{|l|}{ DISCUSSION } \\
\hline $\begin{array}{l}\text { Summary of } \\
\text { evidence }\end{array}$ & 24 & $\begin{array}{l}\text { Summarize the main findings including the strength of evidence } \\
\text { for each main outcome; consider their relevance to key groups } \\
\text { (e.g., healthcare providers, users, and policy makers). }\end{array}$ & 71 \\
\hline Limitations & 25 & $\begin{array}{l}\text { Discuss limitations at study and outcome level (e.g., risk of bias), } \\
\text { and at review-level (e.g., incomplete retrieval of identified } \\
\text { research, reporting bias). }\end{array}$ & 71,73 \\
\hline Conclusions & 26 & $\begin{array}{l}\text { Provide a general interpretation of the results in the context of } \\
\text { other evidence, and implications for future research. }\end{array}$ & 73 \\
\hline \multicolumn{4}{|l|}{ FUNDING } \\
\hline Funding & 27 & $\begin{array}{l}\text { Describe sources of funding for the systematic review and other } \\
\text { support (e.g., supply of data); role of funders for the systematic } \\
\text { review. }\end{array}$ & $n / a$ \\
\hline
\end{tabular}




\section{Appendix B: Search strategy}

We used Medline and Embase (from inception to September 15, 2014) to identify relevant articles. The searches performed in Medline and Embase were essentially similar, except for the subject headings (see below).

\section{Search strategy for Medline}

(("carotid arteries" [MeSH Terms] OR "carotid artery diseases" [MeSH Terms] OR carotid [Tiab] OR local [Tiab]) AND ("elastic modulus" [MeSH Terms] OR "vascular stiffness" [MeSH Terms] OR "compliance" [MeSH Terms] OR wall characteristics [Tiab] OR "elastic modulus" [Tiab] OR disten* OR "compliance" OR stiff*)) AND (("CV diseases" [MeSH Terms] OR ("Cardiovascular" [Tiab] AND ("disease" [MeSH Terms] OR disease* [Tiab] OR event* [Tiab])) OR "atherosclerosis" [MeSH Terms] OR "atherosclerosis" [Tiab]) OR ("stroke" [MeSH Terms] OR "cerebrovascular disorders" [MeSH Terms] OR "ischemic attack, transient" [MeSH Terms] OR ("Cerebrovascular"[Tiab] AND ("disease" [MeSH Terms] OR disease* [Tiab] OR event* [Tiab])) OR ("Transient ischemic" [Tiab] AND (accident* [Tiab] OR attack* [Tiab] OR incident* [Tiab])) OR "coronary disease" [MeSH Terms] OR "myocardial infarction" [MeSH Terms] OR "myocardial ischemia" [MeSH Terms] OR "coronary artery disease" [MeSH Terms] OR "heart diseases" [MeSH Terms] OR ("heart" [Tiab] OR "coronary" [Tiab] OR "myocardial" [Tiab]) AND ("disease" [MeSH Terms] OR disease* [Tiab] OR event* [Tiab] OR "syndrome" [MeSH Terms] OR syndrome* [Tiab])) OR CVD [Tiab] OR CHD [Tiab]) OR (Mortality [MeSH] OR Death [MeSH] OR "Mortality" OR "Death")) AND ("longitudinal studies" [MeSH Terms] OR "prospective studies" [MeSH Terms] OR "cohort studies" [MeSH Terms] OR "follow-up studies" [MeSH Terms] OR "survival" [MeSH Terms] OR Survival Rate [MeSH] OR "Longitudinal" [Tiab] OR "Prospective" [Tiab] OR "Observational" [Tiab] OR "Cohort" [Tiab] OR "Follow-up" [Tiab] OR "Survival analysis" [Tiab])

\section{Search strategy for Embase}

1. $\exp$ CV disease/

2. atherosclerosis/ or carotid atherosclerosis/

3. (CV and (disease* or event*)).ti,ab.

4. 1 or 2 or 3

5. exp cerebrovascular accident/

6. exp transient ischemic attack/

7. (cerebrovascular and (disease* or event*)).ti,ab.

8. (Transient ischemic and (accident* or attack* or incident*)).ti,ab.

9. 5 or 6 or 7 or 8

10. exp coronary artery disease/

11. exp heart infarction/ 
80 | Chapter 4

12. exp heart muscle ischemia/

13. exp heart disease/

14. ((heart or coronary or myocardial) and (disease* or event* or syndrome* or infarct*)).ti,ab.

15. (cvd or chd).ti,ab.

16.10 or 11 or 12 or 13 or 14 or 15

17. exp mortality/

18. exp death/

19. exp survival rate/

20. (mortality or death or survival rate).ti,ab.

21. 17 or 18 or 19 or 20

22.4 or 9 or 16 or 21

23. exp longitudinal study/

24. exp prospective study/

25. exp cohort analysis/

26. exp follow up/

27. exp survival/

28. (longitudinal or prospective or observational or cohort or follow-up or survival analysis).ti,ab.

29.23 or 24 or 25 or 26 or 27 or 28

30. exp carotid artery/

31. carotid.ti,ab.

32. local.ti,ab.

33. exp arterial stiffness/

34. exp artery compliance/

35. exp Young modulus/

36. (wall characteristics or elastic modulus or disten* or compliance or stiff*).ti,ab.

37. (30 or 31 or 32 ) and (33 or 34 or 35 or 36 )

38. 22 and 29 and 37

39. limit 38 to (English)

40. 39 and "Journal: Article".sa_pubt 


\section{Appendix C: Newcastle-Ottawa scale}

For the present study, items 2 and 3 (selection) of the original Newcastle-Ottawa scale $(\mathrm{NOS})^{1}$ for cohort studies were combined. The original items evaluated the quality of the assessment of the exposed and non-exposed cohorts, respectively. In the present study, however, the total study was "exposed" to the risk factor under study (carotid stiffness). A study can be awarded a maximum of one star for each numbered item within the selection and outcome categories. A maximum of two stars can be given for comparability. The individual items of the scale are described below.

\section{Selection}

1) Representativeness of the cohort:
a) truly representative of the general population *
b) somewhat representative of the general population *
c) selected group of users e.g. nurses, volunteers
d) no description of the derivation of the cohort

2) Ascertainment of determinant (carotid stiffness):
a) used validated echotracking technique to assess carotid stiffness *
b) other method
c) no description

3) Demonstration that outcome of interest was not present at start of study (i.e. no prior cardiovascular disease at baseline, or study adjusted for prior cardiovascular disease in the regression analysis)
a) yes *
b) no

\section{Comparability}

1) Comparability of cohorts on the basis of the design or analysis
a) study controls for systolic, pulse and(or) mean blood pressure *
b) study controls for age *

Outcome

1) Assessment of outcome
a) Assessment by health care professional *
b) record linkage *
c) self-report
d) no description

2) Was follow-up long enough for outcomes to occur
a) yes (median/mean follow-up duration $\geq 5$ year) *
b) no 
82 | Chapter 4

3) Adequacy of follow-up of cohorts

a) complete follow-up - all subjects accounted for *

b) subjects lost to follow-up unlikely to introduce bias (small number lost: $>80 \%$ follow-up, or description provided of those lost) *

c) follow up rate $<80 \%$ and no description of those lost to follow-up

d) no statement 


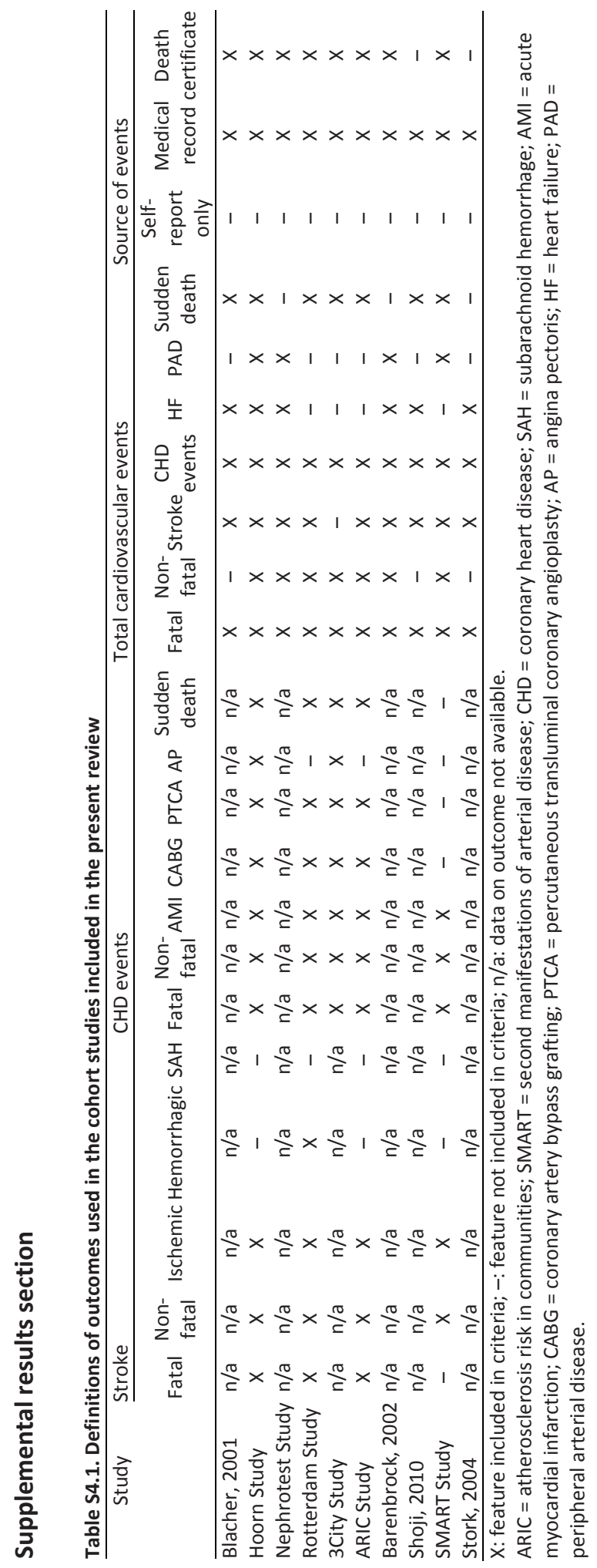




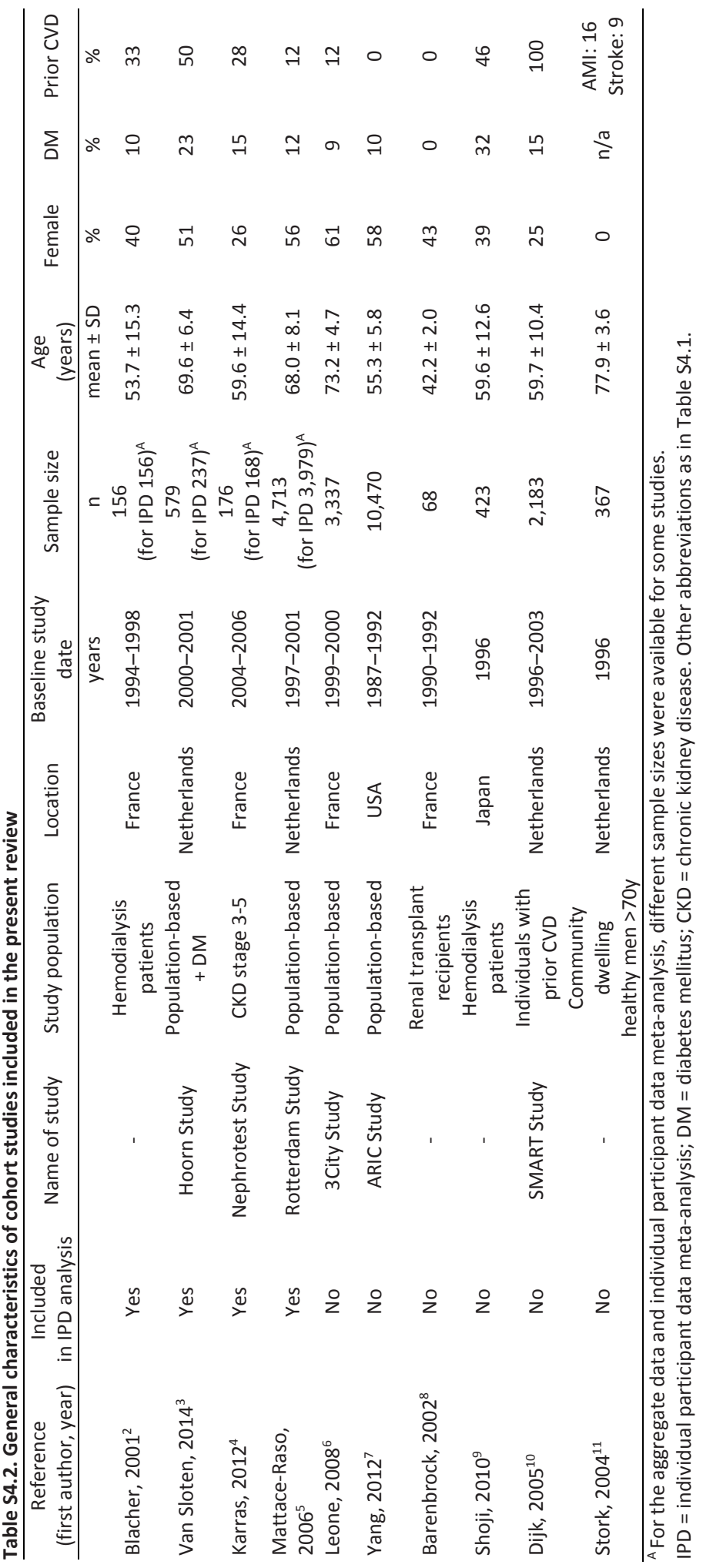




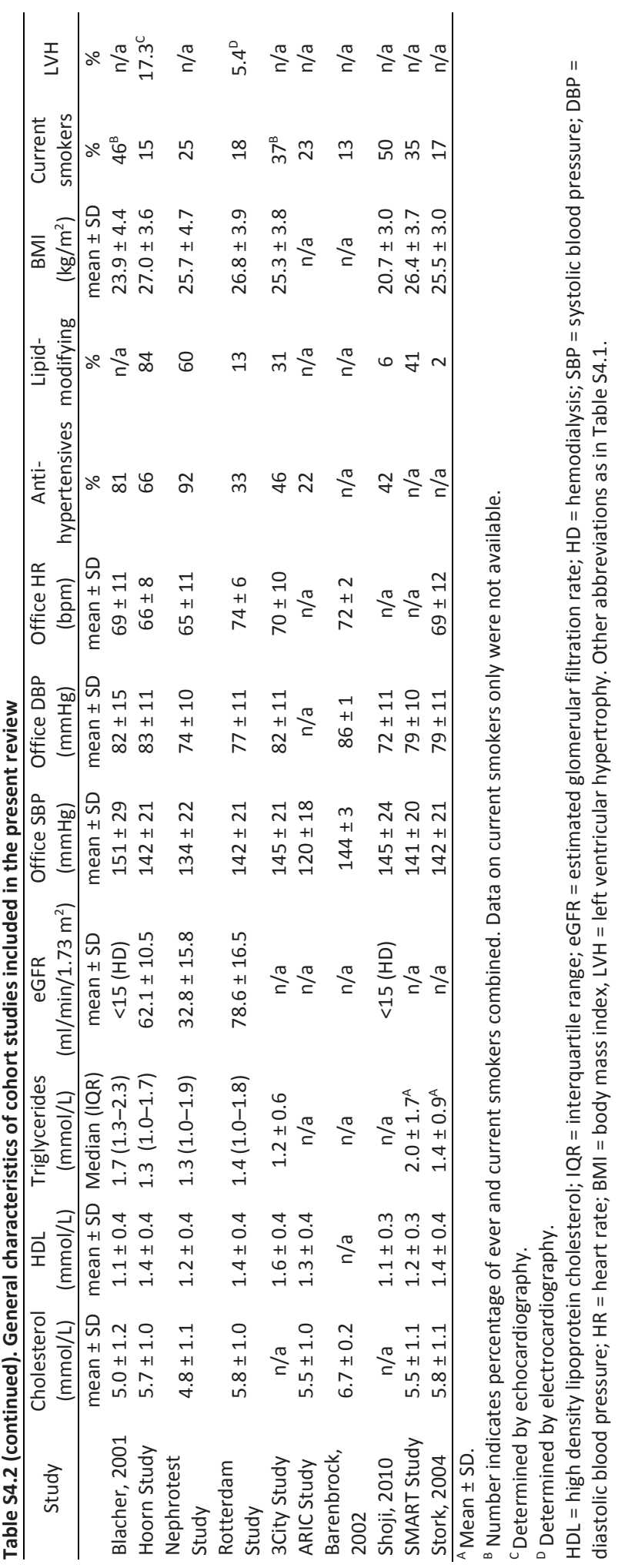




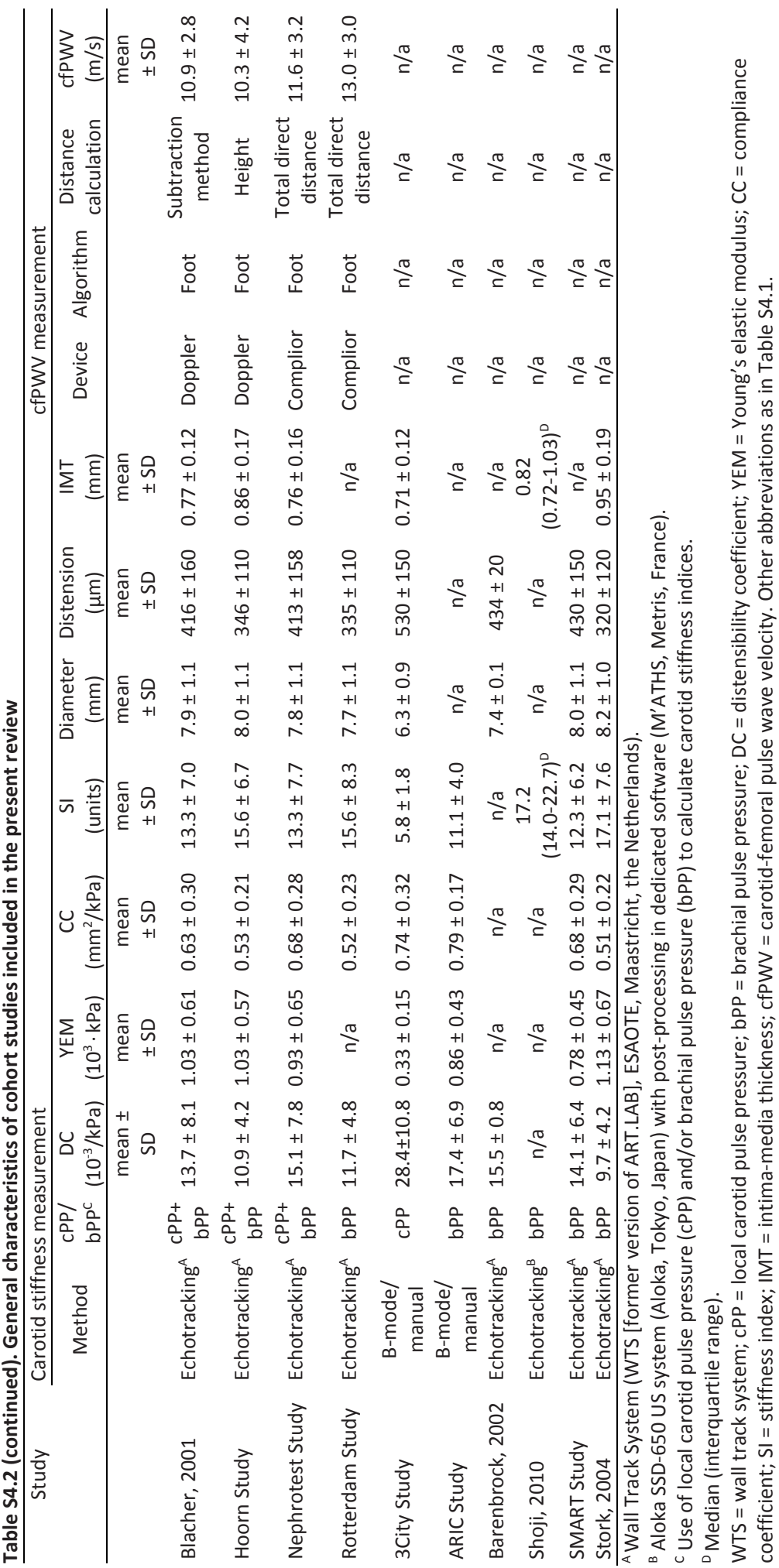




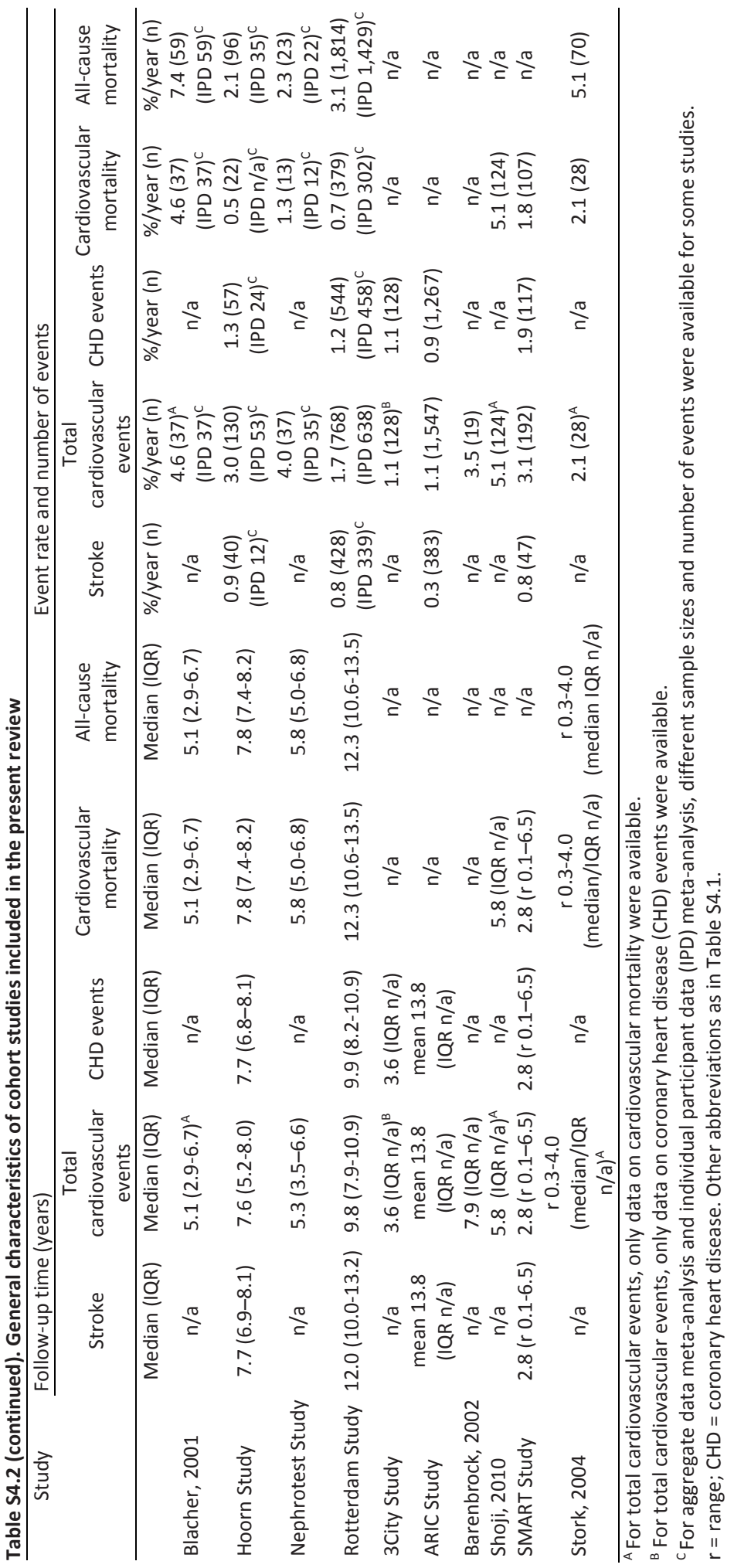


Table S4.3. List of variables adjusted for in the studies included the aggregate data meta-analysis

\begin{tabular}{|c|c|}
\hline Study & Variables adjusted for in the aggregate data-meta-analysis \\
\hline Blacher, 2001 & $\begin{array}{l}\text { Age, sex, MAP, HR, BMI, smoking, DM, triglycerides, total/HDL ratio, anti-hypertensive } \\
\text { medication }\end{array}$ \\
\hline Hoorn Study & $\begin{array}{l}\text { Age, sex, MAP, HR, prior CVD, BMI, smoking, DM, triglycerides, total/HDL cholesterol ratio, } \\
\text { eGFR, albuminuria, physical activity }\end{array}$ \\
\hline Nephrotest Study & $\begin{array}{l}\text { Age, sex, MAP, HR, prior CVD, BMI, smoking, DM, triglycerides, total/HDL cholesterol ratio, } \\
\text { anti-hypertensive and lipid-modifying medication }\end{array}$ \\
\hline Rotterdam Study & $\begin{array}{l}\text { Age, sex, MAP, HR, prior CVD, BMI, smoking, DM, triglycerides, total/HDL cholesterol ratio, } \\
\text { anti-hypertensive and lipid-modifying medication }\end{array}$ \\
\hline 3City Study & $\begin{array}{l}\text { Age, sex, study site, education, MAP, HR, DM, BMI, prior CVD, smoking, LDL cholesterol, } \\
\text { triglycerides, anti-hypertensive and lipid-modifying medication, IMT, carotid plaques }\end{array}$ \\
\hline ARIC Study & $\begin{array}{l}\text { Age, sex, race, study site, SBP, height, weight, smoking, DM, total and HDL cholesterol, anti- } \\
\text { hypertensive medication, IMT }\end{array}$ \\
\hline Barenbrock, 2002 & $\begin{array}{l}\text { Age, sex, } \mathrm{SBP}, \mathrm{DBP}, \mathrm{HR} \text {, smoking, creatinine, total cholesterol, } \mathrm{Hb} \text {, carotid diameter, HD } \\
\text { duration (prior to renal transplantation) }\end{array}$ \\
\hline Shoji, 2010 & $\begin{array}{l}\text { Age, sex, SBP, prior CVD, smoking, DM, HDL cholesterol, creatinine, albumin, PTH, CRP, } \\
\text { calcium carbonate use, HD duration }\end{array}$ \\
\hline SMART Study & Age, age ${ }^{2}$, sex, MAP, smoking, anti-hypertensive medication \\
\hline Stork, 2004 & Age, diuretics, carotid plaques \\
\hline
\end{tabular}

Table S4.4. Newcastle-Ottowa Scale (NOS) scores for included studies in the present review ${ }^{A}$

\begin{tabular}{lccccccccc}
\hline Study & S1 & S2 & S3 & C1a & C1b & O1 & O2 & O3 & Total score \\
\hline Blacher, 2001 & 0 & 1 & 1 & 1 & 1 & 0 & 1 & 1 & 6 \\
Hoorn Study & 1 & 1 & 1 & 1 & 1 & 1 & 1 & 1 & 8 \\
Nephrotest Study & 0 & 1 & 1 & 1 & 1 & 1 & 1 & 1 & 7 \\
Rotterdam Study & 1 & 1 & 1 & 1 & 1 & 1 & 1 & 1 & 8 \\
3City Study & 1 & 0 & 1 & 1 & 1 & 1 & 0 & 1 & 6 \\
ARIC study & 1 & 0 & 1 & 1 & 1 & 1 & 1 & 1 & 7 \\
Barenbrock, 2002 & 0 & 1 & 1 & 1 & 1 & 1 & 1 & 1 & 7 \\
Shoji, 2010 & 0 & 1 & 1 & 1 & 1 & 1 & 1 & 1 & 7 \\
SMART Study & 0 & 1 & 0 & 1 & 1 & 1 & 0 & 1 & 5 \\
Stork, 2004 & 1 & 1 & 0 & 0 & 1 & 1 & 0 & 1 & 5 \\
\hline
\end{tabular}

${ }^{A}$ For an explanation of the individual items on selection (S), comparability (C) and outcome (O), see the provided NOS. Maximal NOS score is 8 . 
Table S4.5. Results of the individual participant meta-analysis. Association between one standard deviation lower carotid distensibility coefficient (DC) and incident cardiovascular events and mortality ${ }^{A}$ : additional adjustments for carotid-femoral pulse wave velocity (cfPWV) (panel A) and analysis of risk improvement (panel B)

\begin{tabular}{|c|c|c|c|c|}
\hline Models & $\begin{array}{l}\text { Coronary heart } \\
\text { disease events }\end{array}$ & $\begin{array}{c}\text { Total cardiovascular } \\
\text { events }\end{array}$ & $\begin{array}{c}\text { Cardiovascular } \\
\text { mortality }\end{array}$ & $\begin{array}{l}\text { All-cause } \\
\text { mortality }\end{array}$ \\
\hline $\begin{array}{l}\text { A. Cox regression } \\
\text { analysis }\end{array}$ & \multicolumn{4}{|c|}{ Hazard ratio ( $95 \%$ confidence interval) } \\
\hline Model $1^{B}$ & $1.02(0.90 ; 1.16)$ & $1.14(0.94 ; 1.37)$ & $1.36(0.95 ; 1.95)$ & $\begin{array}{c}1.34(1.11 ; \\
1.62)\end{array}$ \\
\hline Model $1^{B}+\operatorname{cfPWV}$ & $1.02(0.90 ; 1.16)$ & $1.10(0.92 ; 1.33)$ & $1.39(0.92 ; 2.10)$ & $\begin{array}{c}1.31(1.10 \\
1.57)\end{array}$ \\
\hline $\begin{array}{l}\text { B. Risk improvement } \\
\text { analysis }^{C}\end{array}$ & \multicolumn{4}{|c|}{ Effect estimate ( $95 \%$ confidence interval) } \\
\hline IDI (\%-point) & $-0.0(-0.1 ; 0.1)$ & $0.1(-0.2 ; 0.3)$ & $0.3(0.1 ; 0.5)$ & $0.6(0.4 ; 0.9)$ \\
\hline Continuous NRI (\%) & $3.9(-7.3 ; 15.1)$ & $5.0(-4.6 ; 15.0)$ & $17.5(3.2: 31.7)$ & $\begin{array}{c}19.0 \\
(12.3 ; 25.7)\end{array}$ \\
\hline C-statistic base model & $\begin{array}{c}0.698 \\
(0.673 ; 0.723)\end{array}$ & $\begin{array}{c}0.721 \\
(0.690 ; 0.752)\end{array}$ & $\begin{array}{c}0.812 \\
(0.773 ; 0.851)\end{array}$ & $\begin{array}{c}0.778 \\
(0.760 ; 0.796)\end{array}$ \\
\hline $\begin{array}{l}\text { C-statistic extended } \\
\text { model }\end{array}$ & $\begin{array}{c}0.699 \\
(0.674 ; 0.724)\end{array}$ & $\begin{array}{c}0.721 \\
(0.690 ; 0.752)\end{array}$ & $\begin{array}{c}0.813 \\
(0.774 ; 0.852)\end{array}$ & $\begin{array}{c}0.779 \\
(0.761 ; 0.797)\end{array}$ \\
\hline Change in C-statistic & $\begin{array}{c}0.001 \\
(-0.003 ; 0.005)\end{array}$ & $\begin{array}{c}0.001 \\
(-0.003 ; 0.004) \\
\end{array}$ & $\begin{array}{c}0.001 \\
(-0.003 ; 0.004)\end{array}$ & $\begin{array}{c}0.001 \\
(-0.001 ; 0.003) \\
\end{array}$ \\
\hline
\end{tabular}

\footnotetext{
${ }^{\mathrm{A}}$ Number of participants (n) for incident coronary heart disease events: 4,114 with 482 events and 40,207 person-years of follow-up; for total cardiovascular events: $n=4,395$ with 763 events and 41,060 person-years of follow-up; for cardiovascular mortality: $n=4,540$ with 351 events and 50,711 person-years of follow-up; and for all-cause mortality: $n=4,545$ with 1,545 events and 52,622 person-years of follow-up.

${ }^{B}$ Model 1: results adjusted for age, sex, mean arterial pressure, heart rate, body mass index, smoking habits, diabetes, triglycerides, total / high density lipoprotein cholesterol ratio, prior cardiovascular disease, the use of lipid-modifying and anti-hypertensive medication.

${ }^{\mathrm{C}}$ Base model for risk improvement analysis included Framingham cardiovascular risk score factors and cfPWV. Model was extended by carotid DC (per one lower standard deviation). $\mathrm{IDI}=$ integrated discrimination improvement ; NRI = net reclassification index.
} 

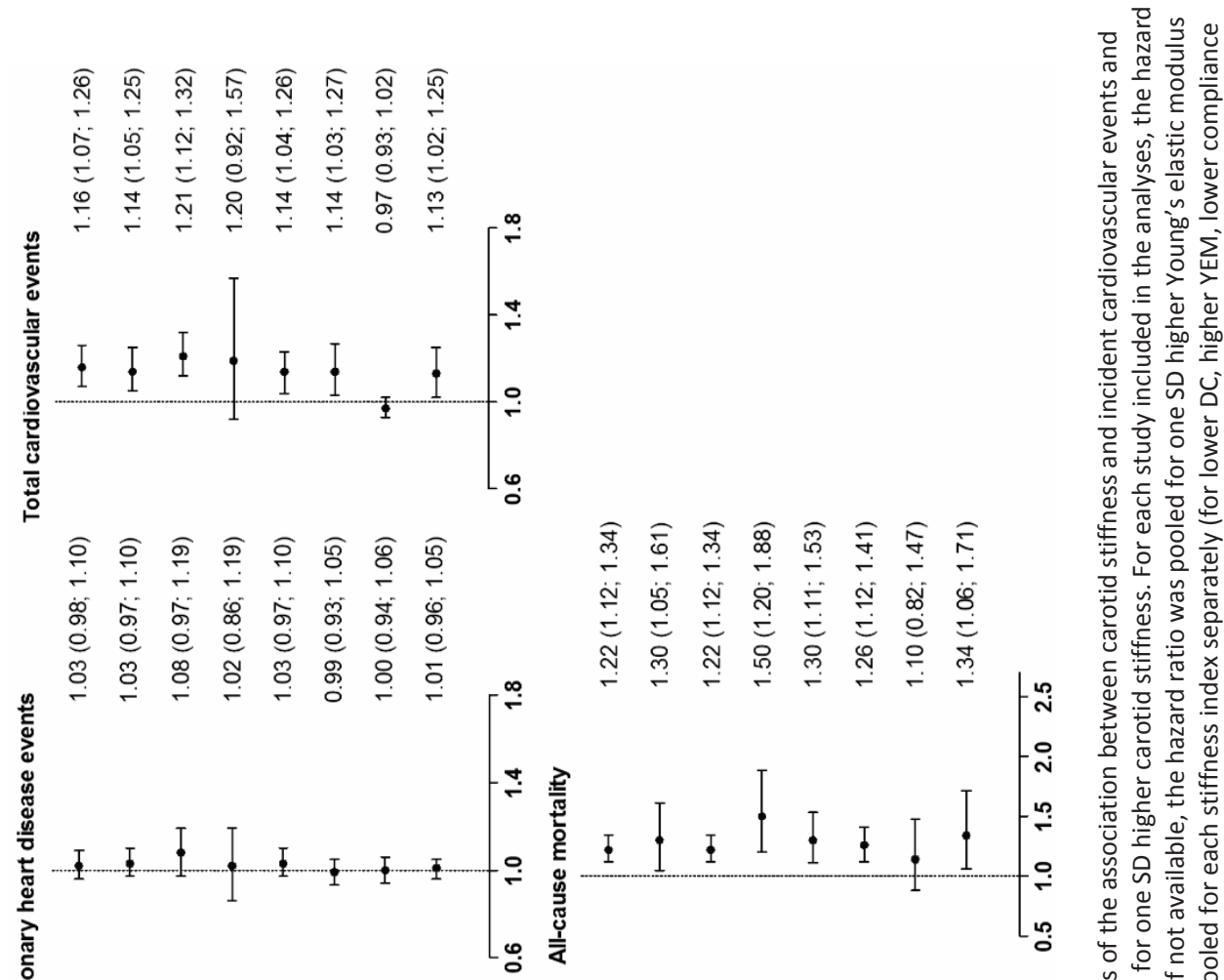

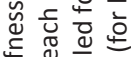

范

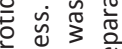

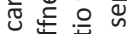

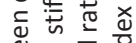

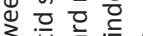

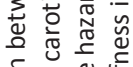

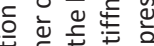

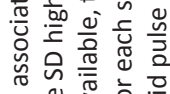

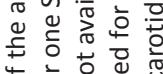
원둥

ㅇํ
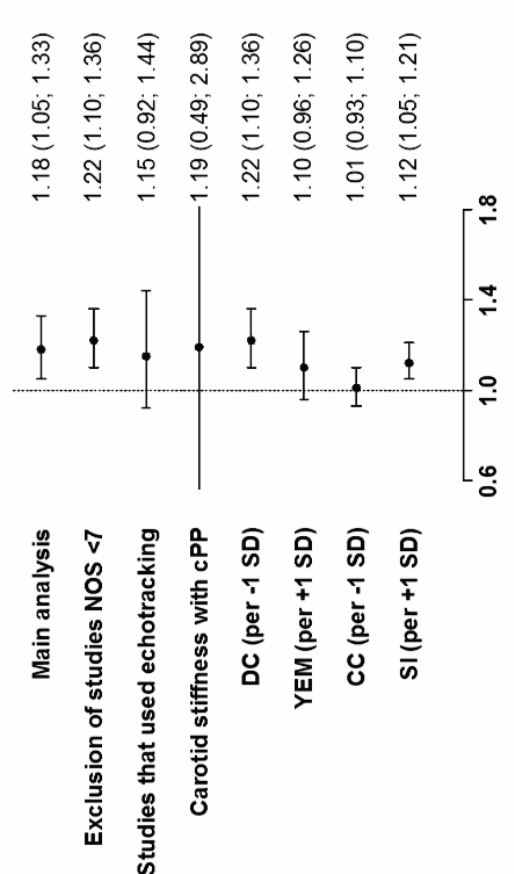
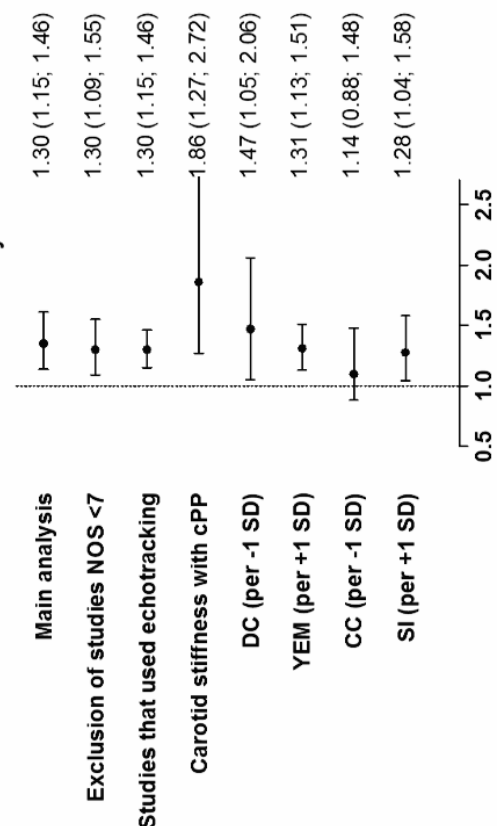

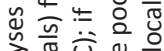

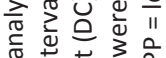
i.

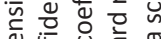

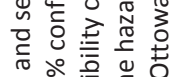
ㄷํำํㅡㄴ 인

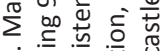

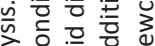
ते 윰율

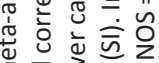
ह 일

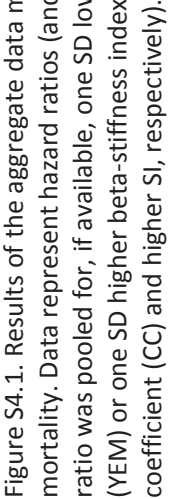




\section{References}

1. Wells GA SB, O'Connell D, Peterson J et al. The Newcastle-Ottawa Scale (NOS) for assessing the quality if nonrandomized studies in meta-analyses. www.ohri.ca/programs/clinical_epidemiology/oxford.htm.

2. Blacher J, Guerin AP, Pannier B, Marchais SJ, London GM. Arterial calcifications, arterial stiffness, and cardiovascular risk in end-stage renal disease. Hypertension. 2001;38:938-942.

3. van Sloten TT, Schram MT, van den Hurk K, et al. Local stiffness of the carotid and femoral artery is associated with incident cardiovascular events and all-cause mortality: the Hoorn study. J Am Coll Cardiol. 2014;63:1739-1747.

4. Karras A, Haymann JP, Bozec E, et al. Large artery stiffening and remodeling are independently associated with all-cause mortality and cardiovascular events in chronic kidney disease. Hypertension. 2012;60:1451-1457.

5. Mattace-Raso FU, van der Cammen TJ, Hofman A, et al. Arterial stiffness and risk of coronary heart disease and stroke: the Rotterdam Study. Circulation. 2006;113:657-663.

6. Leone N, Ducimetiere P, Gariepy J, et al. Distension of the carotid artery and risk of coronary events: the three-city study. Arterioscler Thromb Vasc Biol. 2008;28:1392-1397.

7. Yang EY, Chambless L, Sharrett AR, et al. Carotid arterial wall characteristics are associated with incident ischemic stroke but not coronary heart disease in the Atherosclerosis Risk in Communities (ARIC) study. Stroke. 2012;43:103-108.

8. Barenbrock M, Kosch M, Joster E, Kisters K, Rahn KH, Hausberg M. Reduced arterial distensibility is a predictor of cardiovascular disease in patients after renal transplantation. J Hypertens. 2002;20:79-84.

9. Shoji T, Maekawa K, Emoto M, et al. Arterial stiffness predicts cardiovascular death independent of arterial thickness in a cohort of hemodialysis patients. Atherosclerosis. 2010;210:145-149.

10. Dijk JM, Algra A, van der Graaf Y, Grobbee DE, Bots ML. Carotid stiffness and the risk of new vascular events in patients with manifest cardiovascular disease. The SMART study. Eur Heart J. 2005;26:12131220.

11. Stork S, van den Beld AW, von Schacky C, et al. Carotid artery plaque burden, stiffness, and mortality risk in elderly men: a prospective, population-based cohort study. Circulation. 2004;110:344-348. 


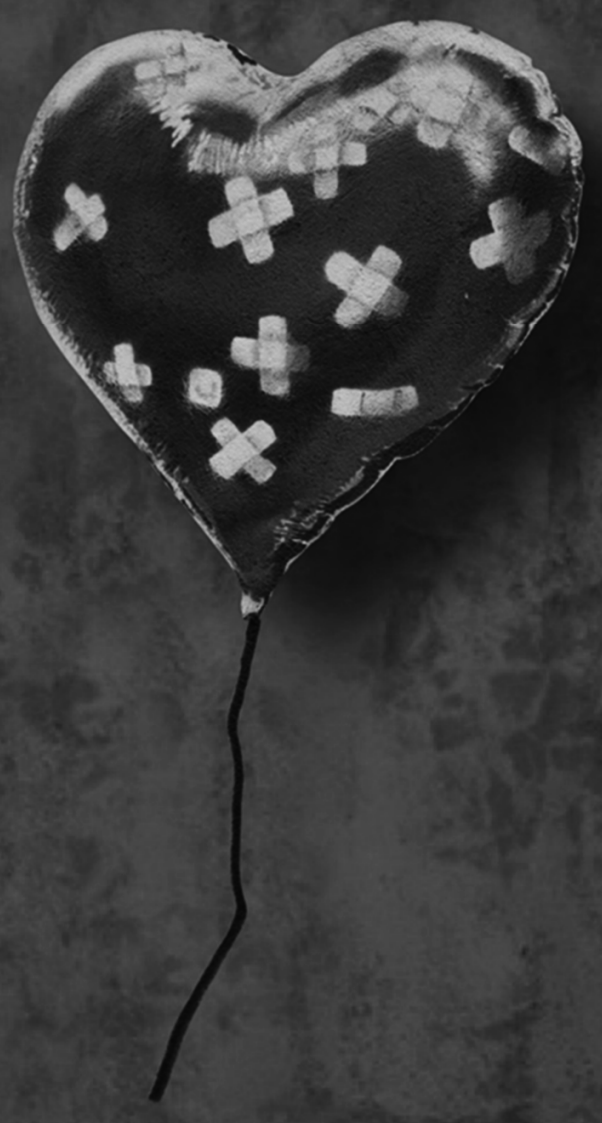

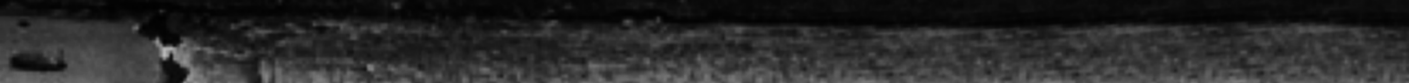
1. 1. 1. 


\section{Chapter 5}

\section{Association between arterial stiffness,}

cerebral small vessel disease and cognitive impairment: a systematic review and meta-analysis

TT van Sloten, AD Protogerou, RMA Henry, MT Schram, L Launer, CDA Stehouwer 
94 | Chapter 5

\section{Abstract}

\section{Background}

Arterial stiffness may be a cause of cerebral small vessel disease and cognitive impairment. We therefore performed a systematic review and meta-analysis of studies on the association between stiffness, cerebral small vessel disease and cognitive impairment.

\section{Methods}

For the associations between stiffness (i.e. carotid-femoral pulse wave velocity (cfPWV), brachial-ankle PWV (baPWV), carotid stiffness and pulse pressure) on the one hand and cerebral small vessel disease and cognitive impairment on the other, we identified 23 ( $n=15,666 ; 20$ cross-sectional; 1 longitudinal; 2 combined cross-sectional/longitudinal) and 41 studies ( $n=57,671 ; 26$ cross-sectional; 11 longitudinal; 4 combined crosssectional/longitudinal), respectively.

\section{Results}

Pooled analysis of cross-sectional studies showed that greater stiffness was associated with markers of cerebral small vessel disease with odds ratios, per one higher standard deviation, of 1.29 to $1.32(\mathrm{P}<.001)$. Studies on cognitive impairment could not be pooled due to large heterogeneity. Nevertheless, these studies showed an association between greater stiffness and cognitive impairment, although the strength of this association was relatively weak.

\section{Conclusion}

The present study supports the hypothesis that greater arterial stiffness is a cause of microvascular brain disease, which may result in cognitive impairment. 


\section{Introduction}

Increased arterial stiffness leads to an increased pulsatile pressure load, which can damage the microcirculation. ${ }^{1,2}$ The brain is more vulnerable for this increased pressure load, because its microcirculation is characterized by low impedance, allowing the pressure load to penetrate deeply into its microvascular bed., ${ }^{1,2}$ In the brain, microvascular damage can manifest itself as white matter hyperintensities (WMH), cerebral microbleeds and lacunar infarcts, ${ }^{3}$ which may ultimately result in cognitive impairment, including dementia. ${ }^{4}$

Currently, consistent evidence is lacking, however, to support an association between increased arterial stiffness on the one hand and cerebral small vessel disease and cognitive impairment on the other, despite the fact that in recent years a growing number of studies have been done on this issue. Existing studies were done in diverse study populations and evaluated different measures of cerebral small vessel disease, cognitive function and arterial stiffness. Measures of arterial stiffness included carotid-femoral pulse wave velocity (cfPWV), brachial-ankle PWV (baPWV) and local distensibility measurements of the carotid artery (i.e. local carotid stiffness). These indices reflect stiffening of different parts of the arterial tree, and may be differentially associated with cerebral small vessel disease and cognitive impairment. In addition, some studies used pulse pressure (PP) (i.e. the difference between systolic and diastolic blood pressure) as a surrogate measure of arterial stiffness. PP is, however, determined by factors other than arterial stiffness, including stroke volume and wave reflections. ${ }^{5}$ This may affect the association between arterial stiffness and cerebral small vessel disease and cognitive impairment.

Three previous reviews $s^{6-8}$ have examined the association between arterial stiffness and microvascular brain disease. However, these studies evaluated only cognitive impairment, ${ }^{6,7}$ included a limited number of measures of arterial stiffness and cognitive impairment, ${ }^{6,7}$ included only studies done in healthy individuals, ${ }^{8}$ did not perform a study quality assessment ${ }^{6-8}$ and/or did not do a meta-analysis. ${ }^{8}$

In view of the above, we performed a systematic review and meta-analysis of the literature on the association between, on the one hand, arterial stiffness (i.e. cfPWV, baPWV, and local carotid stiffness and PP) and, on the other, markers of cerebral small vessel disease and cognitive impairment. 


\section{Methods}

This systematic review and meta-analysis is reported in accordance with preferred reporting items for systematic reviews and meta-analyses (PRISMA) statement (see supplemental material, Appendix A) ${ }^{9}$

\section{Data sources}

We identified relevant studies through a search of Medline and Embase from inception to July 18, 2014 (search terms are provided as supplemental material, Appendix B). In addition, we identified studies by reviewing the reference lists of all relevant articles identified.

\section{Study selection and evaluation procedure}

Two reviewers (TVS and AP) selected independently all relevant studies based on title and abstract, retrieved selected full texts, performed an eligibility assessment and assessed risk of bias (described below). Any disagreements between the reviewers were resolved by consensus.

\section{Eligibility criteria}

Human studies were eligible if they met the following criteria: 1) cross-sectional or longitudinal in design; 2 ) sample size $n \geq 150 ; 2$ ) investigated an association between, on the one hand, arterial stiffness and, on the other, markers of cerebral small vessel disease and/or cognitive function; and 3) measured arterial stiffness by cfPWV, baPWV or local carotid arterial stiffness, and/or measured PP, either at the level of the brachial artery (i.e. peripheral PP) or carotid artery or aorta (i.e. central PP). Case-control studies were excluded. For cerebral small vessel disease, we selected all studies with data on any of the following magnetic resonance imaging (MRI)-detected markers: WMH, cerebral microbleeds and lacunar infarcts. ${ }^{3}$ In addition, most silent infarcts (i.e. infarcts detected in individuals without a history of stroke/transient ischemic attack) and subcortical infarcts (i.e. cerebral infarcts in the deep brain regions not extending into the cortex) are lacunar, ${ }^{3}$ and were also included. Studies that used computed tomography (CT) to detect markers of cerebral small vessel disease were excluded, because CT is less sensitive than MRI. ${ }^{3}$ For cognitive function, we selected all studies with data on any measure of global and/or domain-specific cognitive function. Only papers written in English were included. For studies that published more than one article based upon overlapping groups of participants, with the same outcome measure and study design, we included only the study with the largest number of participants. 


\section{Assessment of risk of bias}

Risk of bias was evaluated with a slightly modified version of the Newcastle Ottawa Scale $(N O S)^{10}$ (NOS is provided as supplemental material, Appendix C). The NOS includes items on participant selection, validity of measurements, whether or not results were adjusted for age, systolic and/or mean blood pressure, and (for studies on cognitive function) education, plus (for longitudinal studies) duration and completeness of follow-up.

\section{Data extraction}

One reviewer (TVS) extracted data with use of a standardized form and a second reviewer (AP) verified data for accuracy and completeness. Any disagreements were resolved by consensus. Information on the following items was extracted from each study: design, sample size, population characteristics, measures of arterial stiffness, cerebral small vessel disease and cognitive impairment, unadjusted and/or adjusted results and variable(s) that were adjusted for in the original analyses. Classification of cognitive domains and included cognitive function tests are described in the supplemental material, Table S5.1. Additional data were requested for two studies ${ }^{11,12}$ from corresponding authors; one ${ }^{12}$ provided the requested data.

\section{Statistical analysis}

We intended to pool results of studies that were sufficiently homogeneous with regard to study methodology and statistical analysis. However, such a meta-analysis was methodologically possible only for the cross-sectional association between cfPWV, baPWV and local carotid stiffness on the one hand and markers of cerebral small vessel disease on the other. Results of studies on PP or cognitive impairment could not be pooled due to a large heterogeneity between studies (see below).

For the meta-analysis, results were pooled for the association between cfPWV, baPWV and local carotid stiffness on the one hand and a categorical measure of cerebral small vessel disease on the other. When more than two categories were present for $\mathrm{WMH}$, we used the odds ratio (OR) for the highest compared to the lowest category. For studies that measured deep and periventricular WMH separately and did not provide a measure of total $\mathrm{WMH}$, we included the results for periventricular $\mathrm{WMH}$ only, because periventricular $\mathrm{WMH}$ is more closely related to total $\mathrm{WMH} .{ }^{13}$ For studies that measured lobar and deep cerebral microbleeds separately and did not provide a measure of total microbleeds, we included the results for deep microbleeds only, because deep microbleeds are more strongly associated with microvascular damage. ${ }^{14}$ Pooled standardized ORs were calculated with the use of the random-effects inverse variance method. If available, we included the fully adjusted value for the OR. Heterogeneity between studies was investigated with Higgins ${ }^{2}$ statistic and Cochran's Q test. An $\mathrm{I}^{2}>50 \%$ and/or a $\mathrm{Q}$ test $\mathrm{P}$-value $<.05$ indicated statistical 
heterogeneity. Funnel plots were used to evaluate potential publication bias. The metaanalysis was performed with Cochrane Review Manager (version 5.2). ${ }^{1515} 15$

\section{Results}

\section{Selection process and study characteristics}

Figure 5.1 shows the selection process of included studies. Of the 23 studies included on cerebral small vessel disease ( $n=15,666 ; 22$ cross-sectional, 3 longitudinal), 8 evaluated cfPWV ( $n=5,017), 7$ baPWV ( $n=3,176), 1$ local carotid stiffness ( $n=912)$ and 12 PP $(n=10,775$; 8 office PP, 2 ambulatory PP, 3 central PP). Of the 41 studies on cognitive impairment ( $n=57,671 ; 30$ cross-sectional, 15 longitudinal), 13 evaluated cfPWV ( $n=12,578), 4$ baPWV $(n=1,313), 1$ local carotid stiffness $(n=3,714)$ and 28 PP $(n=50,408 ; 26$ office PP, 3 central PP). Markers of cerebral small vessel disease studied were WMH (17 studies), microbleeds (6 studies) and infarcts (10 studies). Measures of cognitive function included dementia (8 studies) and tests of global cognitive function (26 studies), memory (14 studies), processing speed (13 studies) and executive function/attention (16 studies). The studies were conducted in the general population, or in selected clinical populations (e.g. individuals with diabetes, hypertension, stroke or Alzheimer's disease) (full study characteristics are provided as supplemental material, Tables S5.2 to S5.5).

\section{A: cerebral small vessel disease}

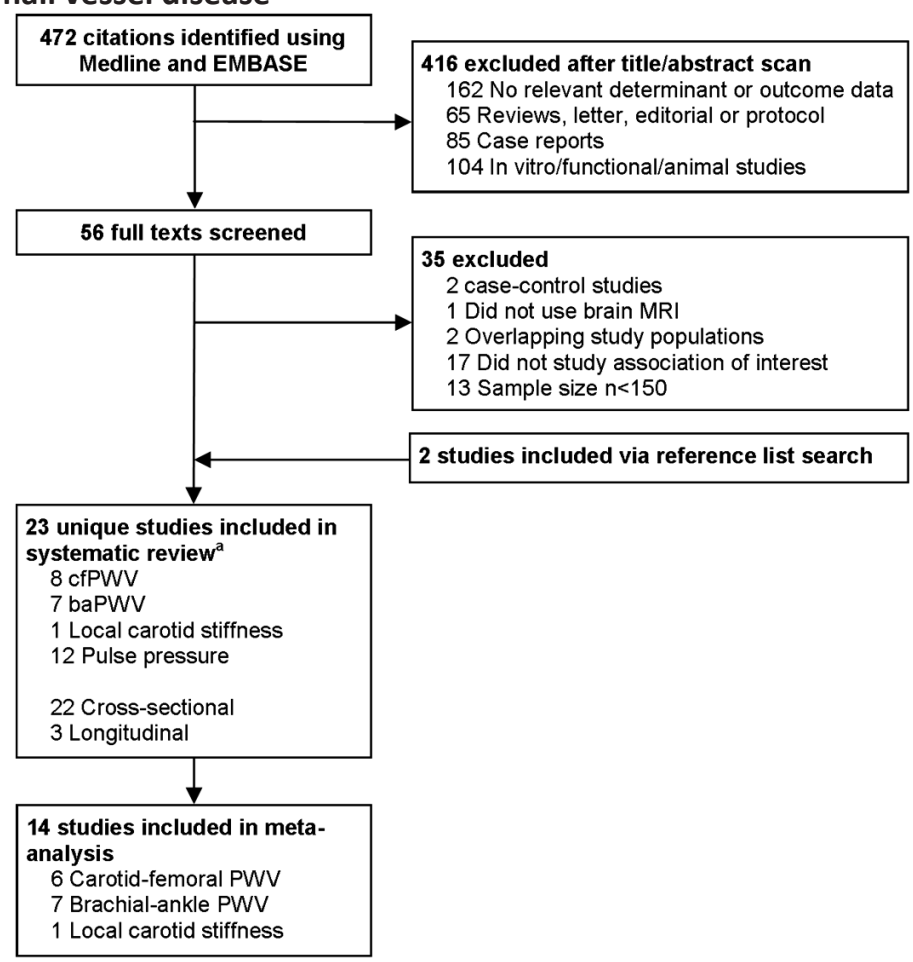




\section{B: cognitive impairment}

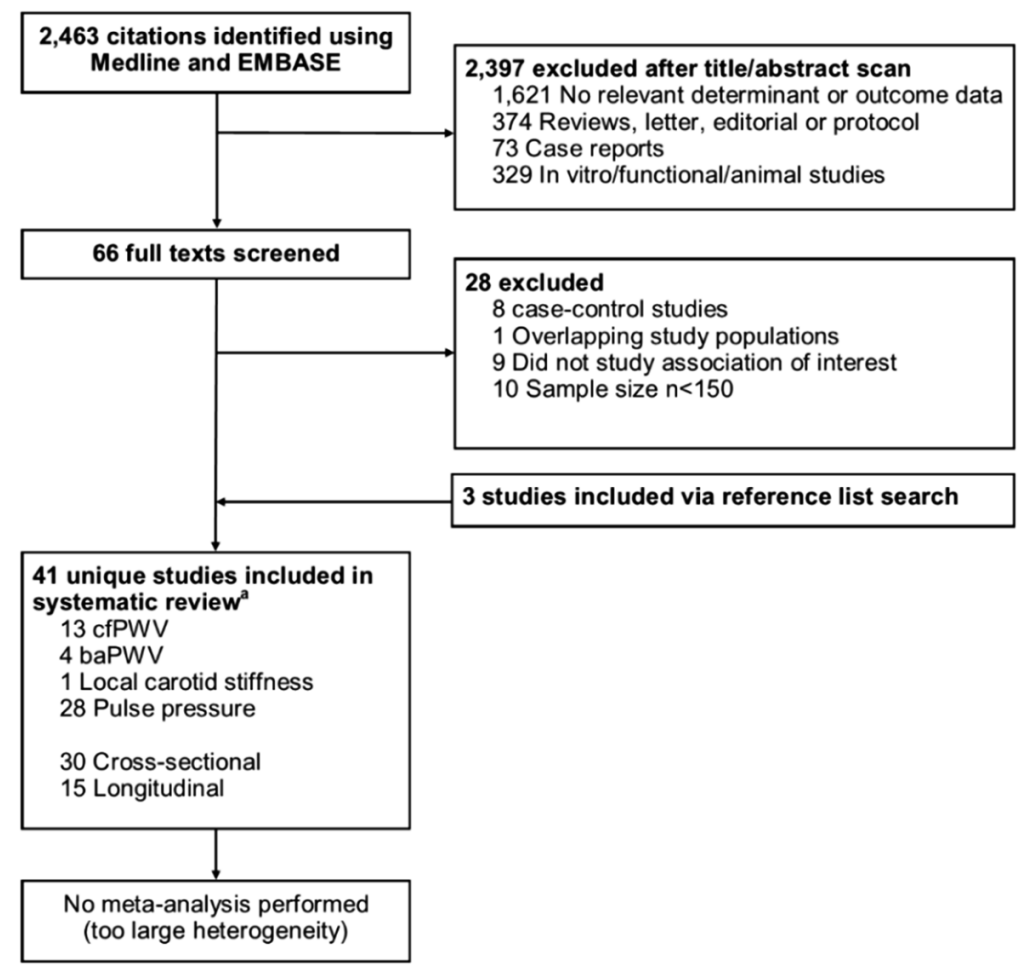

Figure 5.1. Flow diagram of selection process of eligible studies on the association between arterial stiffness and cerebral small vessel disease (panel A) and cognitive impairment (panel B). ${ }^{A}$ Some studies evaluated multiple stiffness indices and included both a cross-sectional and a longitudinal data analysis.

\section{Association between arterial stiffness and cerebral small vessel disease}

\section{CfPWV, baPWV and local carotid stiffness (Figure 5.2)}

Of the fifteen cross-sectional studies included on the association between cfPWV, baPWV and local carotid stiffness on the one hand and markers of cerebral small vessel disease on the other, eleven (73\%) showed a statistically significant association between greater arterial stiffness and cerebral small vessel disease.

The one longitudinal study ${ }^{16}$ (Health Aging and Body Composition study; $n=303$, mean follow-up duration 7 years) showed a significant association between baseline cfPWV and $\mathrm{WMH}$ volume in the left superior longitudinal fasciculus at follow-up (for $\geq$ vs. < median WMH volume in this region, standardized OR 1.47 (95\% confidence interval 1.10 to 1.95$)$ ).

For the pooled analysis, fourteen cross-sectional studies $(n=8,618)$ were included, of which six had measured cfPWV, seven baPWV and one local carotid stiffness. One cross-sectional 
study $(n=184)^{11}$ was excluded from this analysis because this study did not provide sufficient data. The pooled analyses showed that arterial stiffness was statistically significantly associated with WMH (Figure 5.2, panel A), cerebral microbleeds (panel B) and cerebral infarcts (panel C). There was no significant statistical heterogeneity (Figure 5.2). In addition, there was no funnel plot asymmetry (see supplemental material, Figures S5.1). When we performed the pooled analyses separately for baPWV and cfPWV, both indices were associated with markers of cerebral small vessel disease (ORs for +1SD cfPWV 1.39 (1.21 to 1.60) and for +1SD baPWV 1.26 (1.08 to 1.46)) (see supplemental material, Figure S5.2). In addition, when we repeated the analysis after excluding studies with a relatively high risk of bias (NOS score $\leq 3$; two studies), results did not materially change (data not shown).

\section{Study reference $\quad$ Age Odds ratio (95\% confidence interval)}

A. White matter hyperintensities (5 studies)

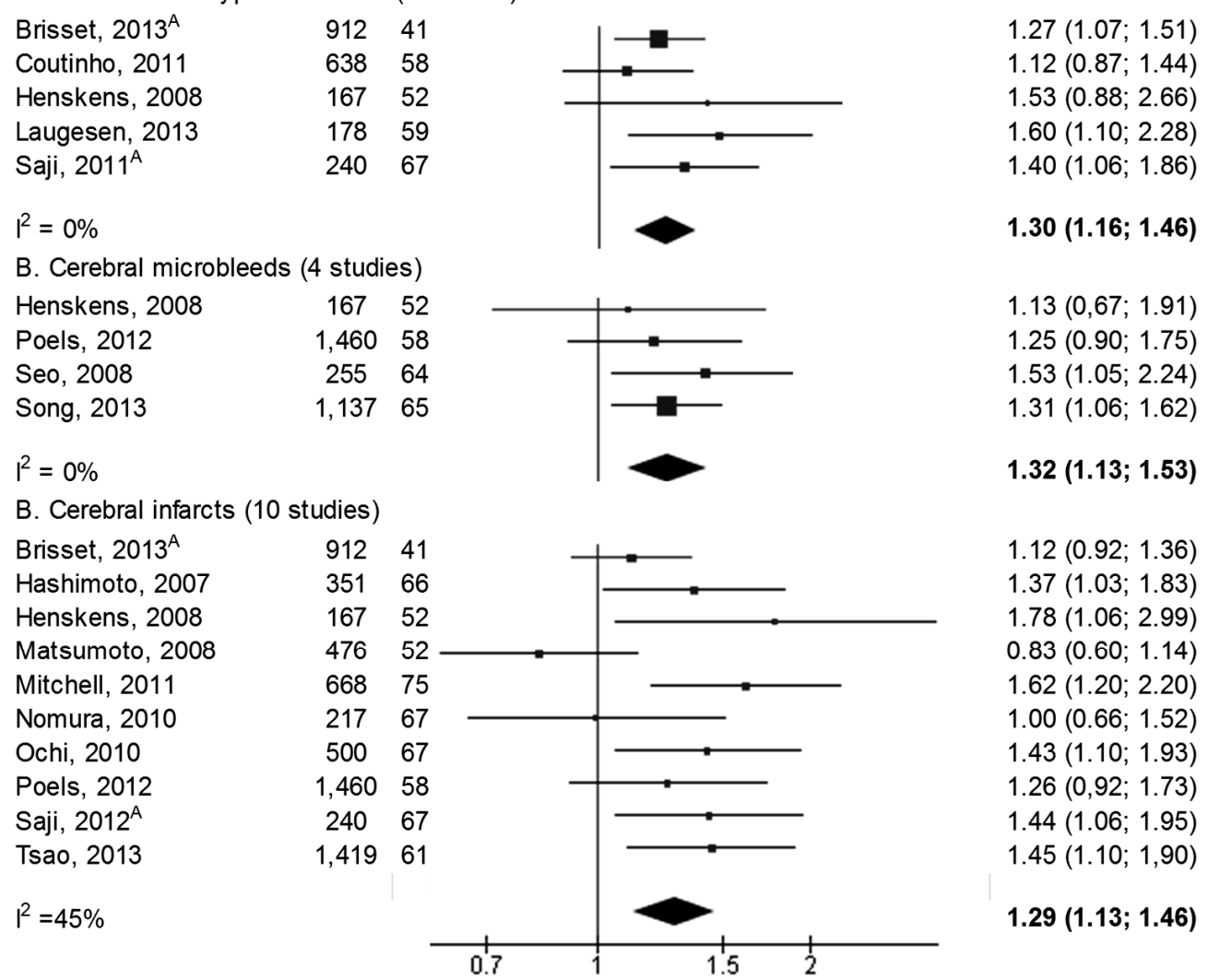

Figure 5.2. Results of the pooled analysis of the association between arterial stiffness and white matter hyperintensities (panel A), cerebral microbleeds (panel B) and cerebral infarcts (panel C). Odds ratios are expressed per one standard deviation (SD) of each stiffness index. ${ }^{A}$ All included studies had adjusted the results for age and mean or systolic blood pressure, with the exception of Brisset, 2013; Ochi, 2010; and Saji, 2011 / 2012, which had not adjusted for mean or systolic blood pressure.

YEM = Young's elastic modulus; cfPWV = carotid-femoral pulse wave velocity; baPWV = brachial-ankle pulse wave velocity. 
Pulse pressure (Table 5.1)

Studies on the association between PP and cerebral small vessel disease differed markedly with regard to the statistical analysis performed. For instance, studies modelled PP as a continuous and categorical variable and calculated standardized and unstandardized effect estimates. Hence, a pooled analysis was not possible. Nevertheless, six of the twelve (50\%) cross-sectional studies included showed a statistically significant association between higher PP and markers of cerebral small vessel disease. The reported standardized regression coefficients ranged from -0.02 to 0.08 , and the standardized ORs from 0.90 to 2.17 .

Of the two longitudinal studies, one ${ }^{17}$ (Rotterdam Study; $n=655$, mean follow-up duration 3.5 years) showed a statistically significant association between higher baseline PP and increase in $\mathrm{WMH}$ over time (regression coefficient per +1 SD PP for decline in $\mathrm{ml} \mathrm{WMH}$ volume/year: -0.04 (0.00; 0.08)). The other study (Van Dooren, 2014) included individuals with hypertension ( $\mathrm{n}=169$; mean follow-up duration 2 years) and showed a not statistically significant association between higher baseline ambulatory PP and progression of CMB over time (standardized OR $1.95(0.95 ; 3.90)$ ). 
Table 5.1. Summary of study results on the association between pulse pressure and cerebral small vessel disease

\begin{tabular}{|c|c|c|c|c|c|c|c|c|}
\hline \multirow[t]{2}{*}{ Reference } & \multirow[t]{2}{*}{ Index } & \multirow[t]{2}{*}{$\mathrm{n}$} & \multirow[t]{2}{*}{$\begin{array}{l}\text { Age } \\
\text { (y) }\end{array}$} & \multicolumn{3}{|c|}{$\begin{array}{l}\text { Standardized effect estimate or direction of } \\
\text { association }^{A}\end{array}$} & \multicolumn{2}{|c|}{ Adjustments } \\
\hline & & & & $W M H$ & Microbleeds & Infarcts & Age & $\begin{array}{l}\text { Blood } \\
\text { pressure }\end{array}$ \\
\hline \multicolumn{9}{|c|}{ Cross-sectional studies (12 studies) } \\
\hline $\begin{array}{l}\text { Poels, } 2010 \text { / } \\
\text { Verhaaren, } 2013^{B}\end{array}$ & off & 3,979 & 60 & $\beta 0.08^{*}$ & OR 1.09 & & $x$ & \\
\hline Tsao, 2013 & cent & 1,419 & 61 & $\beta 0.002$ & & OR 0.97 & $x$ & $x$ \\
\hline Liao, $1997(1)^{\mathrm{C}}$ & off & 843 & 63 & OR 1.32 & & & $x$ & \\
\hline Liao, $1997(2)^{\mathrm{C}}$ & off & 728 & 61 & OR $2.17 *$ & & & $x$ & \\
\hline Aribisala, 2014 & off & 694 & 73 & $\beta 0.04$ & & & $x$ & \\
\hline Kim, 2011 & off & 692 & 63 & $+*$ & & & $x$ & $x$ \\
\hline Mitchell, 2011 & cent & 668 & 75 & $\beta-0.02$ & & OR $1.71^{*}$ & $x$ & $x$ \\
\hline Ochi, $2010^{\mathrm{D}}$ & off & 500 & 67 & & & OR 1.10 & $x$ & \\
\hline Kim, 2012 & off & 236 & 66 & $+*$ & & & $x$ & \\
\hline Naganuma, 2011 & off & 179 & 58 & & OR $1.73^{*}$ & & & $x$ \\
\hline Kwon, 2014 & $a m b$ & 169 & 66 & + & & & $x$ & $x$ \\
\hline Henskens, 2008 & $a m b$ & 167 & 52 & OR 0.90 & OR 0.93 & OR 0.93 & $x$ & $\mathrm{x}$ \\
\hline De Leeuw, 2004 & off & 152 & 68 & + & & & $x$ & \\
\hline \multicolumn{9}{|c|}{ Longitudinal studies (2 studies) } \\
\hline Verhaaren, 2013 & off & 655 & 62 & $+*$ & & & $x$ & \\
\hline Van Dooren, 2014 & $a m b$ & 169 & 53 & & OR 1.95 & & $x$ & \\
\hline
\end{tabular}

Studies are ordered from largest to smallest sample size.

A If available, (fully adjusted) standardized effect estimates are presented. If not, direction of association is presented.

+: higher pulse pressure (PP) associated with higher prevalence/incidence of markers of cerebral small vessel disease

-: higher PP associated with lower prevalence/incidence of markers of cerebral small vessel disease

=: no association between PP and cerebral small vessel disease; direction of association not indicated in original manuscript.

* $\mathrm{P}<.05$

${ }^{B}$ Studies based on an overlapping population (Rotterdam study). Poels, 2010 describes the association between PP and microbleeds in the total population $(n=3,979)$. Verhaaren, 2013 describes in a subsample $(n=655)$ the association between PP and white matter hyperintensities (WMH).

${ }^{c}$ Analyses stratified for European-Americans (1) and African-Americans (2). Results not available for the total study population.

${ }^{D}$ Ochi, 2010 also evaluated the association between office PP and WMH; results for office pulse pressure were qualitatively similar to results for central pulse pressure.

$\mathrm{y}$ = years; $\mathrm{OR}=$ odds ratio; off = office pulse pressure; $\mathrm{amb}=$ ambulatory pulse pressure; cent = central pulse pressure.

\section{Risk of bias and heterogeneity between studies}

Risk of bias among the included studies is presented in detail in Table S5.6 (supplemental material). There was a moderate risk of bias of across the studies on cerebral small vessel disease ( $79 \%$ of the studies scored $\geq 80 \%$ of the total points on the NOS). A lower NOS score was primarily due to the failure to adjust in the analysis for systolic or mean blood pressure (46\% of the studies). In general, the directionality of the effects did not relate to risk of bias. Studies that found a significant association between greater arterial stiffness and cerebral small vessel disease as compared to those studies which did not find such an association did not differ with regard to the variables that they adjusted for in the analysis (i.e. $50 \%$ of significant studies had adjusted for age and blood pressure vs. $54 \%$ of the nonsignificant 
studies, P-value=.83). In addition, there were no differences between significant and nonsignificant studies with regard to sample size (mean $n=778$ vs. $n=521$ ), age of the study population (mean age: 65.5 vs. 60.8 years), type of population studied (56\% and $44 \%$ of significant studies were conducted in general healthy populations and selected clinical populations, respectively, vs. $31 \%$ and $69 \%$ of the nonsignificant studies), and total NOS score (for cross-sectional studies, mean score: 4.1 vs. 3.8 points; for longitudinal studies, mean score: 6.0 vs. 4.0 points) (P-values $>.07)$.

\section{Association between arterial stiffness and cognitive impairment}

CfPWV, baPWV and local carotid stiffness (Table 5.2)

Studies on the association between cfPWV, baPWV and local carotid stiffness on the one hand and cognitive impairment on the other differed markedly with regard to the methodology used. For instance, studies used many different tests of cognitive function and performed different statistical analyses. Hence, a pooled analysis was not possible. Of the eleven cross-sectional studies included on global cognitive impairment (i.e. assessment of dementia or global cognition test), six (55\%) showed a statistically significant association with greater arterial stiffness. In addition, memory was studied in eight cross-sectional studies: two found a statistically significant negative association with greater arterial stiffness. For processing speed, two out of seven, and for executive function/attention, one out of seven cross-sectional studies reported a statistically significant negative association. The reported standardized regression coefficients ranged from -0.05 to 0.20 .

Of the four longitudinal studies included (mean follow-up duration ranged from 1 to 11 years), three showed a significant association between greater arterial stiffness and global cognitive decline. Three longitudinal studies also reported data on cognitive decline in specific domains. Only one study found a statistically significant association between greater arterial stiffness and decline in memory. Associations with cognitive decline in other specific domains (i.e. processing speed and executive function/attention) were not statistically significant. The reported standardized ORs ranged from 0.91 to 1.10. 
Table 5.2. Summary of study results on the association between cfPWV, baPWV and local carotid stiffness on the one hand and cognitive impairment on the other

\begin{tabular}{|c|c|c|c|c|c|c|c|c|c|c|}
\hline \multirow[t]{2}{*}{ Reference } & \multirow[t]{2}{*}{ Index } & \multirow[t]{2}{*}{$\mathrm{n}$} & \multirow{2}{*}{$\begin{array}{l}\text { Age } \\
\text { (y) }\end{array}$} & \multicolumn{5}{|c|}{ Standardized effect estimate or direction of association ${ }^{A}$} & \multicolumn{2}{|c|}{ Adjustments } \\
\hline & & & & Dementia & $\begin{array}{l}\text { Global } \\
\text { score }\end{array}$ & Memory & $\begin{array}{c}\text { Processing } \\
\text { speed }\end{array}$ & $\mathrm{EF} / \mathrm{A}$ & Age & $\begin{array}{c}\text { Blood } \\
\text { pressure }\end{array}$ \\
\hline \multicolumn{11}{|c|}{ Cross-sectional studies (15 studies) } \\
\hline Poels, $2007^{B}$ & cfPWV & 3,714 & 72 & & + & & + & $+*$ & $x$ & $x$ \\
\hline Tsao, 2013 & cfPWV & 1,578 & 61 & & & $\beta-0.01$ & & $\beta 0.07$ & $x$ & $x$ \\
\hline Zhong, 2013 & cfPWV & 1,433 & 75 & & $+*$ & $+^{*}$ & + & + & $x$ & \\
\hline Mitchell, 2011 & cfPWV & 668 & 75 & & & $\beta 0.10^{*}$ & $\beta-0.03$ & $\beta 0.08$ & $x$ & $x$ \\
\hline Watson, 2011 & cfPWV & 552 & 73 & & $\beta 0.11^{*}$ & $\beta 0.07$ & $\beta 0.12 *$ & & $x$ & $x$ \\
\hline Elias, 2009 & cfPWV & 409 & 61 & & $=$ & $=$ & $\beta 0.13^{*}$ & & $x$ & $x$ \\
\hline Muller, 2007 & cfPWV & 396 & 60 & & & $\beta 0.01$ & $\beta 0.02$ & $\beta 0.01$ & $x$ & \\
\hline Sugawara, 2010 & baPWV & 388 & 69 & & + & & & & $x$ & $x$ \\
\hline Kim, 2009 & baPWV & 370 & 55 & & $=$ & & & & & \\
\hline Fuijwara, 2005 & baPWV & 352 & 77 & & $+*$ & & & & $x$ & $x$ \\
\hline Singer, 2013 & cfPWV & 319 & 80 & & $\beta-0.05$ & $\beta 0.20$ & $\beta 0.05$ & $\beta-0.03$ & $x$ & $x$ \\
\hline Hanon, 2005 & cfPWV & 308 & 78 & OR $2.63^{*}$ & $+^{*}$ & & & & $x$ & $x$ \\
\hline Scuteri, 2013 & cfPWV & 280 & 78 & & $+*$ & & & & $x$ & \\
\hline Fukuhara, 2006 & baPWV & 203 & 85 & & $+*$ & & & & $x$ & $x$ \\
\hline $\begin{array}{l}\text { Kearney- } \\
\text { Schwartz, } 2009\end{array}$ & cfPWV & 198 & 69 & & & $=$ & & $=$ & & \\
\hline \multicolumn{11}{|c|}{ Longitudinal studies (4 studies) } \\
\hline Poels, $2007^{\mathrm{B}}$ & cfPWV & 2,767 & 71 & HR 0.91 & OR $0.93^{C}$ & & OR $1.09^{C}$ & OR $1.10^{C}$ & $x$ & $x$ \\
\hline $\begin{array}{l}\text { Zeki Al Hazzouri, } \\
2013 / \\
\text { Watson, } 2011^{\text {D }}\end{array}$ & cfPWV & 2,488 & 74 & & $+^{*}$ & + & - & & $x$ & $x$ \\
\hline Benetos, 2012 & cfPWV & 873 & 88 & & $+*$ & & & & $x$ & $x$ \\
\hline Waldstein, 2008 & cfPWV & 582 & 54 & & $+*$ & $+*$ & + & + & $\mathrm{x}$ & $\mathrm{x}$ \\
\hline
\end{tabular}

Studies are ordered from largest to smallest sample size.

A If available, (fully adjusted) standardized effect estimates are presented. If not, direction of association is presented. Mean effect estimates are presented if multiple results were available for the same cognitive domain.

+ : greater arterial stiffness associated with worse cognitive function

-: greater arterial stiffness associated with better cognitive function

=: no association between arterial stiffness and cognitive function; direction of association not indicated in original manuscript.

*: $\mathrm{P}<.05$

${ }^{B}$ Poels, 2007 also evaluated the association between carotid stiffness and cognitive impairment; results for carotid stiffness were qualitatively similar to results for carotid-femoral pulse wave velocity (cfPWV).

${ }^{\mathrm{C}}$ Cognitive decline specified as >-1SD change of the mean difference between examinations.

${ }^{D}$ Studies based on an overlapping study population (health, aging, and body composition study). Zeki Al Hazzouri, 2013 describes the association between cfPWV and global cognitive decline in the total population $(n=2,488)$. Watson, 2011 describes in a subsample $(n=522)$ the association between cfPWV and global cognitive decline, memory and processing speed.

$y=$ years; $E F / A$ = executive function/attention; $O R$ = odds ratio; $H R$ = hazard ratio; carotid = local carotid stiffness; baPWV = brachial-ankle pulse wave velocity.

\section{Pulse pressure (Table 5.3)}

In general, studies on the association between PP and cognitive impairment had more heterogeneous results than those on cfPWV, baPWV and local carotid stiffness and cognitive impairment. Of the thirteen cross-sectional studies included on global cognitive impairment, four (31\%) showed a statistically significant association with greater PP. In addition, memory was studied in seven cross-sectional studies: four found a statistically 
significant negative association with greater PP. For processing speed, two out of six, and for executive function/attention, two out of nine cross-sectional studies reported a statistically significant negative association. The reported standardized regression coefficients ranged from -0.02 to 0.16 . In contrast, three studies ${ }^{18-20}$ found a statistically significant association, but in the opposite direction, i.e. between higher PP and better (global and(or) domain-specific) cognitive function (Table 5.3). These studies were done in individuals with Alzheimer's disease ${ }^{18,20}$ or in the oldest old (i.e. individuals $\geq 85$ years). ${ }^{19}$

Of the thirteen longitudinal studies on global cognitive decline (mean duration of follow-up ranged from 1 to 14 years), three (23\%) showed a statistically significant association with greater PP. In addition, three longitudinal studies also reported data on cognitive decline in specific domains. All studies found a significant association with decline in executive function/attention and one with decline in memory, but none found a statistically significant association with processing speed. In addition, one study ${ }^{21}$ found a U-shaped association between PP and cognitive decline, whereas one study ${ }^{22}$ found a statistically significant association between higher PP and lower cognitive decline. The latter two studies were both done in older individuals (mean age 82 and 85 years, respectively) (Table 5.3). The reported standardized ORs ranged from 0.85 to 1.21 . 
Table 5.3. Summary of study results on the association between pulse pressure and cognitive impairment

\begin{tabular}{|c|c|c|c|c|c|c|c|c|c|c|}
\hline \multirow[t]{2}{*}{ Reference } & \multirow[t]{2}{*}{ Index } & \multirow[t]{2}{*}{$\mathrm{n}$} & \multirow[t]{2}{*}{$\begin{array}{l}\text { Age } \\
\text { (y) }\end{array}$} & \multicolumn{5}{|c|}{$\begin{array}{c}\text { Standardized effect estimate or direction of } \\
\text { association }{ }^{A}\end{array}$} & \multicolumn{2}{|c|}{ Adjustments } \\
\hline & & & & Dementia & $\begin{array}{l}\text { Global } \\
\text { score }\end{array}$ & Memory & $\begin{array}{l}\text { Processing } \\
\text { speed }\end{array}$ & $E F / A$ & Age & $\begin{array}{c}\text { Blood } \\
\text { pressure }\end{array}$ \\
\hline \multicolumn{11}{|c|}{ Cross-sectional studies (17 studies) } \\
\hline Tsivgoulis, 2009 & off & 19,836 & 65 & & OR 0.98 & & & & $x$ & \\
\hline Obisesan, 2008 & off & 5,408 & 71 & & $+*$ & & & & $\mathrm{x}$ & \\
\hline Tsao, 2013 & cent & 1,578 & 61 & & & $\beta 0.07^{*}$ & & $\beta 0.02$ & $x$ & $x$ \\
\hline Robbins, 2005 & off & 1,563 & 49 & & $+*$ & $+*$ & $+*$ & $=$ & $x$ & $x$ \\
\hline Mitchell, 2011 & cent & 668 & 75 & & & $\beta 0.11^{*}$ & $\beta-0.02$ & $\beta 0.09$ & $\mathrm{x}$ & $x$ \\
\hline Davis, 2003 & off & 609 & 74 & & $-*$ & $-*$ & $=$ & -* & $\mathrm{x}$ & \\
\hline Sabayan, 2012 & off & 572 & 85 & & - & & & & $x$ & \\
\hline Chrysohoou, 2012 & off & 535 & 75 & & OR $1.41^{*}$ & & & & $x$ & \\
\hline Pase, $2013^{B}$ & cent & 493 & 53 & & & $\beta 0.12^{*}$ & $\beta 0.14^{*}$ & $\beta 0.16^{*}$ & $x$ & $x$ \\
\hline Molander, 2010 & off & 476 & 90 & & -* & & & & $x$ & \\
\hline Fuijwara, 2005 & off & 352 & 77 & & $+*$ & & & & $\mathrm{x}$ & \\
\hline Yasar, 2011 & off & 337 & 74 & & - & $=$ & + & + & $x$ & \\
\hline $\begin{array}{l}\text { van Bruchem- } \\
\text { Visser, } 2009\end{array}$ & off & 327 & 77 & & -* & & & & $x$ & \\
\hline Giang, 2005 & off & 314 & 63 & & + & + & + & - & $x$ & \\
\hline Kalaitzidis, 2013 & off & 256 & 53 & & + & & & $+*$ & $x$ & \\
\hline Fukuhara, 2006 & off & 203 & 85 & & + & & & & $x$ & $x$ \\
\hline Raz, 2011 & off & 158 & 52 & & & & & $=$ & $\mathrm{x}$ & \\
\hline \multicolumn{11}{|c|}{ Longitudinal studies (13 studies) } \\
\hline Peters, 2013 & off & 3,337 & 84 & $+*$ & & & & & & \\
\hline Freitag,2006 (1) ${ }^{c}$ & off & 2,505 & 58 & + & & & & & $\mathrm{x}$ & \\
\hline Freitag,2006 (2) ${ }^{C}$ & off & 2,505 & 77 & - & & & & & $x$ & \\
\hline Lee, 2013 & off & 1,925 & 73 & & + & & & & $x$ & $x$ \\
\hline Ogunniyi, 2011 & off & 1,753 & 76 & OR $1.21 *$ & & & & & $x$ & \\
\hline Waldstein, 2008 & off & 1,749 & 57 & & $+^{*}$ & $+*$ & + & $+^{*}$ & $\mathrm{x}$ & $x$ \\
\hline Taylor, 2013 & off & 1,484 & 50 & & - & & & & $x$ & \\
\hline Qiu, 2003 & off & 1,270 & 82 & $\mathrm{U}^{* \mathrm{D}}$ & & & & & $x$ & $x$ \\
\hline Benetos, 2012 & off & 873 & 88 & & $=$ & & & & $\mathrm{x}$ & $x$ \\
\hline Morris, 2001 & off & 634 & 72 & OR 0.85 & & & & & $x$ & \\
\hline McFall, 2014 & off & 599 & 71 & & & & & $+*$ & & \\
\hline Yang, 2011 & off & 594 & 76 & OR 1.00 & & & & & $x$ & \\
\hline Sabayan, 2012 & off & 572 & 85 & & -* & & & & $x$ & \\
\hline Yasar, 2011 & off & 337 & 74 & & + & + & + & $+*$ & $x$ & \\
\hline
\end{tabular}

Studies are from largest to smallest sample size.

A If available, (fully adjusted) standardized effect estimates are presented. If not, direction of association is presented. Mean effect estimates are presented if multiple results were available for the same cognitive domain. + : higher PP associated with worse cognitive function

-: higher PP associated with better cognitive function

=: no association between PP and cognitive function; direction of association not indicated in original manuscript.

*: $\mathrm{P}<.05$

${ }^{B}$ Pase, 2013 also evaluated the association between office PP and cognitive impairment; results for office PP were qualitatively similar to results for central PP.

${ }^{\text {C }}$ Study evaluated PP measured at a mean age of 58 (1) and 72 years (2), respectively.

${ }^{\mathrm{D}} \mathrm{U}$-shaped association between PP and cognitive decline.

Abbreviations as in Table 5.2 . 


\section{Risk of bias and heterogeneity between studies}

There was a moderate risk of bias of across the studies on cognitive impairment (62\% of the studies scored $\geq 80 \%$ of the total points on the NOS) (individual NOS scores are provided as supplemental material, Table S5.6). A lower NOS score was primarily due to the failure to adjust in the analysis for systolic or mean blood pressure (52\% of the studies). Studies that found a significant association between arterial stiffness and cognitive impairment as compared to those studies which did not find such an association had a smaller sample size (mean $n=1,190$ vs. $n=1,737$ ), but this difference was not statistically significant (P-value $=.49$ ). There were no differences between significant and nonsignificant studies with regard to the variables that they adjusted for in the analysis (i.e. $58 \%$ of significant studies had adjusted for age, education and blood pressure vs. 39\% of the nonsignificant studies), age of the study population (mean age: 70.0 vs. 71.4 years), type of population studied (62\% and $38 \%$ of significant studies were conducted in general healthy populations and selected clinical populations, respectively, vs. $79 \%$ and $21 \%$ of the nonsignificant studies), total NOS score (for cross-sectional studies, mean score: 3.9 vs. 3.6 points; for longitudinal studies, mean score: 6.0 vs. 6.8 points) and, for longitudinal studies, duration of follow-up (7.5 vs. 7.9 years) ( $P$-values $>.13)$. 


\section{Discussion}

\section{Main findings}

The present systematic review and meta-analysis had two main findings. First, with regard to the systematic review, most studies showed an independent association between greater arterial stiffness, as measured by cfPWV, baPWV, local carotid stiffness and PP, and markers of cerebral small vessel disease. In addition, studies found an association between higher cfPWV, baPWV and local carotid stiffness on the one hand and cognitive impairment on the other, but the strength of this association was relatively weak. In addition, results on the association between PP and cognitive impairment were inconsistent. Second, with regard to the meta-analysis, pooled analysis showed a statistically significant and strong association between greater arterial stiffness and markers of cerebral small vessel disease.

\section{Association between arterial stiffness and cerebral small vessel disease}

The results of the pooled analysis of cross-sectional studies showed that higher cfPWV, baPWV and local carotid stiffness were statistically significantly associated with markers of cerebral small vessel disease with standardized ORs of 1.29 to 1.32. These results strongly suggests that arterial stiffness is an important risk indicator of microvascular disease, with a strength of the association comparable to that of the association between arterial stiffness and measures of atherosclerosis (e.g. ankle-brachial index and coronary calcium score ${ }^{5}$ ).

\section{Association between arterial stiffness and cognitive impairment}

Studies also showed an association between higher cfPWV, baPWV and local carotid stiffness on the one hand and cognitive impairment on the other. Estimated effect sizes were, however, relatively small and this association was statistically significant in a relatively low number of studies. This relatively weak association may be due to the fact that mechanisms other than microvascular disease play a role in the pathobiology of cognitive impairment, including neurodegenerative pathology and the process of atherothrombosis.

In addition, PP was less consistently associated with cognitive impairment than cfPWV, baPWV and local carotid stiffness. Some studies showed an association between higher PP and worse cognitive function, whereas other studies found an inverted association, i.e. higher PP was associated with better cognitive function. These studies ${ }^{18-20,22}$ were all done in (biologically) older individuals. The presence of an inverted association in these individuals may have several explanations. Possibly, cognitive impairment or the process of neurodegeneration may cause dysregulation of blood pressure and, thereby, a decline in PP. ${ }^{23}$ Alternatively, in frail older individuals low blood pressure (including low PP) may, as a result of a dysfunctional vascular system, comprise perfusion of the brain, which may manifest as cognitive impairment. ${ }^{23}$ 


\section{Methodological considerations}

Some methodological issues warrant consideration. First, the associations found might have been overestimated due to publication bias. The funnel plot of studies on the association between arterial stiffness and cerebral small vessel disease did not show (substantial) asymmetry (such asymmetry may indicate the presence of publication bias). However, studies on the association between arterial stiffness and cognitive impairment that found a significant association as compared to those studies which did not find such association had a relatively smaller sample size. This may indicate the presence of publication bias, and suggests that the association between arterial stiffness and cognitive impairment may have been overestimated in earlier research. Second, most of the included studies were crosssectional by design, which precludes a conclusion about the temporality of the studied associations. Nevertheless, the few studies with a longitudinal design did show that greater arterial stiffness was present before the occurrence of cerebral small vessel disease and cognitive impairment. Third, a relatively high number of the studies did not adjust for systolic or mean blood pressure. Blood pressure is an important confounder in the association between arterial stiffness and microvascular disease. In those studies that did adjust for blood pressure, however, the association between arterial stiffness and cerebral small vessel disease/cognitive impairment remained. Fourth, results of studies on PP or cognitive impairment could not be pooled due to a large heterogeneity. Fifth, due to the design of the present systematic review and (aggregate-data) meta-analysis, it was not possible to make a direct comparison between different stiffness indices with regard to the strength of their association with cerebral small vessel disease/cognitive impairment. Sixth, only a limited number of studies measured local carotid stiffness, ambulatory PP or central PP. Evidence for an association between these indices and microvascular brain disease is, therefore, weak and this issue requires further study. Finally, it is not fully clear why some studies found a significant association between arterial stiffness and cerebral small vessel disease/cognitive impairment and others did not. There were no differences between significant and nonsignificant studies with regard to the populations studied (i.e. general healthy populations versus selected clinical populations and age of the study population) and risk of bias (NOS scores).

\section{Assumptions underlying the studied associations}

Important assumptions underlying the associations evaluated in the present review are that markers of cerebral small vessel disease are valid indicators of cerebral microvascular damage, and that microvascular damage is involved in the pathobiology of cognitive impairment. Previous studies $3,24-26$ have indeed demonstrated that markers of cerebral small vessel disease represent both abnormal cerebral microvascular structure and function. For instance, studies have demonstrated that $\mathrm{WMH}$, cerebral microbleeds and lacunar infarcts are associated with disruption and a greater permeability of the blood-brain layer as well as arteriosclerotic changes in small arteries/arterioles. In addition, previous 
studies ${ }^{1,3,4,27}$ have shown a consistent association between cerebral small vessel disease and cognitive impairment.

\section{Underlying mechanisms}

The mechanism that may underlie the observed associations is that greater arterial stiffness leads to microcirculatory damage via an increased pulsatile pressure load. This increased pressure load may directly cause cerebral microvascular damage, despite blood-pressurerelated protective autoregulatory mechanisms. ${ }^{1,2}$ Alternatively, the increased pressure load may induce a microvascular remodelling response, which initially serves to limit the penetration of the pressure load on the microcirculatory system by raising vascular resistance. Yet, this protective response may ultimately become unfavourable, leading to impaired vasoreactivity and microvascular ischemia. It is, moreover, likely that these mechanisms operate simultaneously.

\section{Conclusion}

The present systematic review and meta-analysis shows a consistent association across different cross-sectional studies between greater arterial stiffness and markers of cerebral small vessel disease. This supports the hypothesis that greater arterial stiffness is a cause of microvascular brain disease. In addition, the present review shows that greater arterial stiffness is associated with cognitive impairment, but the strength of this association was relatively weak, and might have been overestimated due to publication bias. Arterial stiffness may be a therapeutic target for the prevention of microvascular brain disease and cognitive impairment. However, further well-powered longitudinal studies are warranted that investigate the temporality of the association between arterial stiffness, cerebral small vessel disease and cognitive impairment. 


\section{References}

1. Mitchell GF. Effects of central arterial aging on the structure and function of the peripheral vasculature: implications for end-organ damage. J Appl Physiol (1985). 2008;105:1652-1660.

2. O'Rourke MF, Safar ME. Relationship between aortic stiffening and microvascular disease in brain and kidney: cause and logic of therapy. Hypertension. 2005;46:200-204.

3. Wardlaw JM, Smith EE, Biessels GJ, et al. Neuroimaging standards for research into small vessel disease and its contribution to ageing and neurodegeneration. Lancet Neurol. 2013;12:822-838.

4. Mitchell GF, van Buchem MA, Sigurdsson S, et al. Arterial stiffness, pressure and flow pulsatility and brain structure and function: the Age, Gene/Environment Susceptibility--Reykjavik study. Brain. 2011;134:3398-3407.

5. Safar ME, O'Rourke RM. Arterial stiffness in hypertension. Amsterdam: Elsevier; 2006.

6. Pase MP, Herbert A, Grima NA, Pipingas A, O'Rourke MF. Arterial stiffness as a cause of cognitive decline and dementia: a systematic review and meta-analysis. Intern Med J. 2012;42:808-815.

7. Rabkin SW, Jarvie G. Comparison of vascular stiffness in vascular dementia, Alzheimer dementia and cognitive impairment. Blood pressure. 2011;20:274-283.

8. Singer J, Trollor JN, Baune BT, Sachdev PS, Smith E. Arterial stiffness, the brain and cognition: a systematic review. Ageing Res Rev. 2014;15:16-27.

9. Moher D, Liberati A, Tetzlaff J, Altman DG. Preferred reporting items for systematic reviews and metaanalyses: the PRISMA statement. Ann Intern Med. 2009;151:264-269, W264.

10. Wells GA SB, O'Connell D, Peterson J et al. The Newcastle-Ottawa Scale (NOS) for assessing the quality if nonrandomized studies in meta-analyses. www.ohri.ca/programs/clinical_epidemiology/oxford.htm.

11. Kearney-Schwartz A, Rossignol P, Bracard S, et al. Vascular structure and function is correlated to cognitive performance and white matter hyperintensities in older hypertensive patients with subjective memory complaints. Stroke. 2009;40:1229-1236.

12. Nomura K, Hamamoto Y, Takahara S, et al. Relationship between carotid intima-media thickness and silent cerebral infarction in Japanese subjects with type 2 diabetes. Diabetes Care. 2010;33:168-170.

13. DeCarli C, Fletcher E, Ramey V, Harvey D, Jagust WJ. Anatomical mapping of white matter hyperintensities (WMH): exploring the relationships between periventricular $\mathrm{WMH}$, deep $\mathrm{WMH}$, and total WMH burden. Stroke. 2005;36:50-55.

14. Poels MM, Vernooij MW, Ikram MA, et al. Prevalence and risk factors of cerebral microbleeds: an update of the Rotterdam scan study. Stroke. 2010;41:S103-106.

15. Review Manager (RevMan) [Computer program]. Version 5.2. Copenhagen: The Nordic Cochrane Centre, The Cochrane Collaboration, 2012.

16. Rosano C, Watson N, Chang Y, et al. Aortic pulse wave velocity predicts focal white matter hyperintensities in a biracial cohort of older adults. Hypertension. 2013;61:160-165.

17. Verhaaren BF, Vernooij MW, de Boer R, et al. High blood pressure and cerebral white matter lesion progression in the general population. Hypertension. 2013;61:1354-1359.

18. Davis RN, Massman PJ, Doody RS. Effects of blood pressure on neuropsychological functioning in Alzheimer's disease. Arch Clin Neuropsychol. 2003;18:19-32.

19. Molander L, Gustafson Y, Lovheim H. Low blood pressure is associated with cognitive impairment in very old people. Dement Geriatr Cogn Disord. 2010;29:335-341.

20. van Bruchem-Visser RL, Mattace-Raso FU, van der Cammen TJ. High systolic and pulse pressure levels are associated with better cognitive performance in patients with probable Alzheimer's disease: a crosssectional observational study in a geriatric outpatient population. Dement Geriatr Cogn Disord. 2009;28:320-324.

21. Qiu C, Winblad B, Viitanen M, Fratiglioni L. Pulse pressure and risk of Alzheimer disease in persons aged 75 years and older: a community-based, longitudinal study. Stroke. 2003;34:594-599. 


\section{2 | Chapter 5}

22. Sabayan B, Oleksik AM, Maier AB, et al. High blood pressure and resilience to physical and cognitive decline in the oldest old: the Leiden 85-plus Study. J Am Geriatr Soc. 2012;60:2014-2019.

23. Muller M, Smulders YM, de Leeuw PW, Stehouwer CD. Treatment of Hypertension in the Oldest Old: A Critical Role for Frailty? Hypertension. 2013.

24. Wardlaw JM, Smith C, Dichgans M. Mechanisms of sporadic cerebral small vessel disease: insights from neuroimaging. Lancet Neurol. 2013;12:483-497.

25. Fisher CM. Capsular infarcts: the underlying vascular lesions. Arch Neurol. 1979;36:65-73.

26. Young VG, Halliday GM, Kril JJ. Neuropathologic correlates of white matter hyperintensities. Neurology. 2008;71:804-811.

27. Gorelick PB, Scuteri A, Black SE, et al. Vascular contributions to cognitive impairment and dementia: a statement for healthcare professionals from the american heart association/american stroke association. Stroke. 2011;42:2672-2713. 


\section{Supplemental Material}

\section{Supplemental methods section}

\section{Appendix A: PRISMA 2009 checklist}

\begin{tabular}{|c|c|c|c|}
\hline Section/topic & & Checklist item & $\begin{array}{l}\text { Reported on } \\
\text { page: }\end{array}$ \\
\hline \multicolumn{4}{|l|}{ TITLE } \\
\hline Title & 1 & Identify the report as a systematic review, meta-analysis, or both. & 93 \\
\hline \multicolumn{4}{|l|}{ ABSTRACT } \\
\hline $\begin{array}{l}\text { Structured } \\
\text { summary }\end{array}$ & 2 & $\begin{array}{l}\text { Provide a structured summary including, as applicable: } \\
\text { background; objectives; data sources; study eligibility criteria, } \\
\text { participants, and interventions; study appraisal and synthesis } \\
\text { methods; results; limitations; conclusions and implications of key } \\
\text { findings; systematic review registration number. }\end{array}$ & 94 \\
\hline \multicolumn{4}{|c|}{ INTRODUCTION } \\
\hline Rationale & 3 & $\begin{array}{l}\text { Describe the rationale for the review in the context of what is } \\
\text { already known. }\end{array}$ & 95 \\
\hline Objectives & 4 & $\begin{array}{l}\text { Provide an explicit statement of questions being addressed with } \\
\text { reference to participants, interventions, comparisons, outcomes, } \\
\text { and study design (PICOS). }\end{array}$ & 95 \\
\hline \multicolumn{4}{|l|}{ METHODS } \\
\hline $\begin{array}{l}\text { Protocol and } \\
\text { registration }\end{array}$ & 5 & $\begin{array}{l}\text { Indicate if a review protocol exists, if and where it can be } \\
\text { accessed (e.g., Web address), and, if available, provide } \\
\text { registration information including registration number. }\end{array}$ & - \\
\hline $\begin{array}{l}\text { Eligibility } \\
\text { criteria }\end{array}$ & 6 & $\begin{array}{l}\text { Specify study characteristics (e.g., PICOS, length of follow-up) and } \\
\text { report characteristics (e.g., years considered, language, } \\
\text { publication status) used as criteria for eligibility, giving rationale. }\end{array}$ & 96 \\
\hline $\begin{array}{l}\text { Information } \\
\text { sources }\end{array}$ & 7 & $\begin{array}{l}\text { Describe all information sources (e.g., databases with dates of } \\
\text { coverage, contact with study authors to identify additional } \\
\text { studies) in the search and date last searched. }\end{array}$ & 96 \\
\hline Search & 8 & $\begin{array}{l}\text { Present full electronic search strategy for at least one database, } \\
\text { including any limits used, such that it could be repeated. }\end{array}$ & \begin{tabular}{|l|} 
Supplemental \\
Material
\end{tabular} \\
\hline $\begin{array}{l}\text { Study } \\
\text { selection }\end{array}$ & 9 & $\begin{array}{l}\text { State the process for selecting studies (i.e., screening, eligibility, } \\
\text { included in systematic review, and, if applicable, included in the } \\
\text { meta-analysis). }\end{array}$ & 96 \\
\hline $\begin{array}{l}\text { Data } \\
\text { collection } \\
\text { process }\end{array}$ & 10 & $\begin{array}{l}\text { Describe method of data extraction from reports (e.g., piloted } \\
\text { forms, independently, in duplicate) and any processes for } \\
\text { obtaining and confirming data from investigators. }\end{array}$ & 96,97 \\
\hline Data items & 11 & $\begin{array}{l}\text { List and define all variables for which data were sought (e.g., } \\
\text { PICOS, funding sources) and any assumptions and simplifications } \\
\text { made. }\end{array}$ & 97 \\
\hline $\begin{array}{l}\text { Risk of bias in } \\
\text { individual } \\
\text { studies }\end{array}$ & $\begin{array}{r}12 \\
\end{array}$ & $\begin{array}{l}\text { Describe methods used for assessing risk of bias of individual } \\
\text { studies (including specification of whether this was done at the } \\
\text { study or outcome level), and how this information is to be used in } \\
\text { any data synthesis. }\end{array}$ & $\begin{array}{l}\text { 97, } \\
\text { Supplemental } \\
\text { Material }\end{array}$ \\
\hline
\end{tabular}




\begin{tabular}{|c|c|c|c|}
\hline $\begin{array}{l}\text { Summary } \\
\text { measures }\end{array}$ & 13 & $\begin{array}{l}\text { State the principal summary measures (e.g., risk ratio, difference } \\
\text { in means). }\end{array}$ & 97 \\
\hline $\begin{array}{l}\text { Synthesis of } \\
\text { results }\end{array}$ & 14 & $\begin{array}{l}\text { Describe the methods of handling data and combining results of } \\
\text { studies, if done, including measures of consistency (e.g., } \mathrm{I}^{2} \text { ) for } \\
\text { each meta-analysis. }\end{array}$ & $97-98$ \\
\hline $\begin{array}{l}\text { Risk of bias } \\
\text { across studies }\end{array}$ & 15 & $\begin{array}{l}\text { Specify any assessment of risk of bias that may affect the } \\
\text { cumulative evidence (e.g., publication bias, selective reporting } \\
\text { within studies). }\end{array}$ & $\begin{array}{l}97, \\
\text { Supplemental } \\
\text { Material }\end{array}$ \\
\hline $\begin{array}{l}\text { Additional } \\
\text { analyses }\end{array}$ & 16 & $\begin{array}{l}\text { Describe methods of additional analyses (e.g., sensitivity or } \\
\text { subgroup analyses, meta-regression), if done, indicating which } \\
\text { were pre-specified. }\end{array}$ & $\begin{array}{l}97,100 \\
\text { Supplemental } \\
\text { Material }\end{array}$ \\
\hline \multicolumn{4}{|l|}{ RESULTS } \\
\hline $\begin{array}{l}\text { Study } \\
\text { selection }\end{array}$ & 17 & $\begin{array}{l}\text { Give numbers of studies screened, assessed for eligibility, and } \\
\text { included in the review, with reasons for exclusions at each stage, } \\
\text { ideally with a flow diagram. }\end{array}$ & Figure 5.1 \\
\hline $\begin{array}{l}\text { Study } \\
\text { characteristics }\end{array}$ & 18 & $\begin{array}{l}\text { For each study, present characteristics for which data were } \\
\text { extracted (e.g., study size, PICOS, follow-up period) and provide } \\
\text { the citations. }\end{array}$ & $\begin{array}{l}98, \text { Tables } \\
\text { S5.2-S5.5 }\end{array}$ \\
\hline $\begin{array}{l}\text { Risk of bias } \\
\text { within studies }\end{array}$ & 19 & $\begin{array}{l}\text { Present data on risk of bias of each study and, if available, any } \\
\text { outcome level assessment (see item 12). }\end{array}$ & \begin{tabular}{|l|}
$102-103,107$ \\
Table S5.6 \\
\end{tabular} \\
\hline \begin{tabular}{l|} 
Results of \\
individual \\
studies
\end{tabular} & 20 & $\begin{array}{l}\text { For all outcomes considered (benefits or harms), present, for each } \\
\text { study: (a) simple summary data for each intervention group (b) } \\
\text { effect estimates and confidence intervals, ideally with a forest } \\
\text { plot. }\end{array}$ & $\begin{array}{l}\text { Figure 5.2, } \\
\text { Tables 5.1- } \\
5.3\end{array}$ \\
\hline $\begin{array}{l}\text { Synthesis of } \\
\text { results }\end{array}$ & 21 & $\begin{array}{l}\text { Present results of each meta-analysis done, including confidence } \\
\text { intervals and measures of consistency. }\end{array}$ & $\begin{array}{l}100, \text { Figures } \\
5.2 \text { and S5.2 } \\
\end{array}$ \\
\hline $\begin{array}{l}\text { Risk of bias } \\
\text { across studies }\end{array}$ & 22 & $\begin{array}{l}\text { Present results of any assessment of risk of bias across studies } \\
\text { (see Item 15). }\end{array}$ & \begin{tabular}{|l|}
$102-103,107$ \\
Figure S5.1 \\
\end{tabular} \\
\hline \begin{tabular}{l|} 
Additional \\
analysis
\end{tabular} & 23 & $\begin{array}{l}\text { Give results of additional analyses, if done (e.g., sensitivity or } \\
\text { subgroup analyses, meta-regression [see Item 16]). }\end{array}$ & $\begin{array}{l}\text { 100, Figure } \\
\text { S5.2 }\end{array}$ \\
\hline \multicolumn{4}{|l|}{ DISCUSSION } \\
\hline $\begin{array}{l}\text { Summary of } \\
\text { evidence }\end{array}$ & 24 & $\begin{array}{l}\text { Summarize the main findings including the strength of evidence } \\
\text { for each main outcome; consider their relevance to key groups } \\
\text { (e.g., healthcare providers, users, and policy makers). }\end{array}$ & 108 \\
\hline Limitations & 25 & $\begin{array}{l}\text { Discuss limitations at study and outcome level (e.g., risk of bias), } \\
\text { and at review-level (e.g., incomplete retrieval of identified } \\
\text { research, reporting bias). }\end{array}$ & 109 \\
\hline Conclusions & 26 & $\begin{array}{l}\text { Provide a general interpretation of the results in the context of } \\
\text { other evidence, and implications for future research. }\end{array}$ & 110 \\
\hline \multicolumn{4}{|l|}{ FUNDING } \\
\hline Funding & 27 & $\begin{array}{l}\text { Describe sources of funding for the systematic review and other } \\
\text { support (e.g., supply of data); role of funders for the systematic } \\
\text { review. }\end{array}$ & $n / a$ \\
\hline
\end{tabular}




\section{Appendix B: Search strategy}

Two searches were done in which the search terms for arterial stiffness were combined with those for cerebral small vessel disease and cognitive impairment, respectively. All terms were searched in the title and abstract. Search terms are provided below.

\section{Search terms Medline}

Arterial stiffness: elasticity [MeSH] OR vascular stiffness [MeSH] OR elastic modulus [MeSH] OR pulse wave analysis [MeSH] OR arterial compliance OR distensibility OR elastic modulus OR elasticity OR stiffness OR pulse wave velocity OR pulse pressure Cerebral small vessel disease: cerebral small vessel diseases [MeSH] OR stroke, lacunar [MeSH] OR leukoaraiosis [MeSH] OR white matter OR leukoencephalopathy OR leukoaraiosis OR lacun* OR ((infarct* OR stroke*) AND (subcortical OR silent OR small vessel)) OR ((microbleed* OR microhemorrhage OR dot-like hemosiderin) AND (cerebral OR Brain))

Cognitive impairment: cognition [MeSH] OR cognition disorders [MeSH] OR mild cognitive impairment [MeSH] OR dementia [MeSH] OR cognit* OR neurocognit* OR neuropsychologic* OR memory OR dementia OR Alzheimer*

\section{Search terms Embase}

Arterial stiffness: blood vessel parameters [exp] OR pulse wave [exp] OR arterial compliance OR distensibility OR elastic modulus OR elasticity OR stiffness OR pulse wave velocity OR pulse pressure Cerebral small vessel disease: cerebral small vessel disease [mp] OR lacunar stroke [exp] OR leukoaraiosis [exp] OR leukoencephalopathy [exp] OR white matter lesion [exp] OR white matter OR leukoencephalopathy OR leukoaraiosis OR lacun* OR ((infarct* OR stroke*) AND (deep OR subcortical OR silent)) OR ((microbleeds OR microhemorrhage OR dot-like hemosiderin) AND (cerebral OR brain))

Cognitive impairment: cognitive defect [exp] OR mild cognitive impairment [exp] OR Alzheimer disease [exp] OR neuropsychological test [exp] OR memory [exp] OR dementia [exp] OR cognition [exp] OR cognit* OR neurocognitive OR neuropsychologic* OR memory OR dementia OR Alzheimer* 


\section{Appendix C: Newcastle-Ottawa scale (NOS)}

For the present study, items 2 and 3 (selection) of the original NOS for cohort studies were combined (for the individual items, see below). The original items evaluated the quality of the assessment of the exposed and nonexposed cohorts, respectively. In the present study, however, the total study was "exposed" to the risk factor under study (i.e. arterial stiffness). For cross-sectional studies, the same NOS was used as applied for longitudinal studies, but items 4 (selection) and 2 and 3 (outcome) were not scored. A study can be awarded a maximum of one star for each numbered item within the selection and outcome categories. A maximum of two stars can be given for comparability. The individual items of the scale are described below.

\section{Selection}

1) Representativeness of the cohort
a) truly representative of the general population *
b) somewhat representative of the general population *
c) selected group of users e.g. nurses, volunteers
d) no description of the derivation of the cohort

2) Ascertainment of determinant (arterial stiffness)
a) use of an approved device, for cfPWV: Spyghmocor, Complior, Vicorder, Pulsetrace, Pulsepen, Millar tonometry, Cardiovascular Engineering tonometry; for baPWV: VP- 1000, VP-2000, Form ABI/PWV, VS-2000; for local carotid stiffness: wall-tracking echo device; for brachial pulse pressure: validated device (see www.dableducational.com for validated devices); for central pulse pressure: Spyghmocor, Millar tonometry, HEM9000, Cardiovascular Engineering tonometry *
b) not an approved device
c) no description

3) Demonstration that outcome of interest was not present at start of study (at start of the study no or lower burden of cerebral small vessel disease or better performance on cognitive function tests)
a) yes *
b) no

\section{Comparability}

1) Comparability of cohorts on the basis of the design or analysis
a) study controls for systolic and(or) mean blood pressure *
b) study controls for age and (for studies evaluating cognitive impairment) education * 


\section{Outcome}

1) Assessment of outcome

a) Objective measurements *

- $\quad$ For cerebral small vessel disease: MRI scanner with a field strength of 1.5

Tesla or higher and the following (minimal) sequences: for white matter

hyperintensities: T2-weighted and fluid-attenuated inversion recovery (FLAIR);

for lacunar/subcortical infarcts: T1- and(or) T2-weighted; for cerebral

microbleeds: T2*-weighted gradient echo sequence

- For cognitive function: validated cognitive tests

b) record linkage (for dementia only) *

c) self-report

d) no description

2) Was follow-up long enough for outcomes to occur
a) yes (median/mean follow-up duration $>4$ year) *
b) no

3) Adequacy of follow-up of cohorts
a) complete follow-up - all subjects accounted for *
b) subjects lost to follow-up unlikely to introduce bias: $>80 \%$ follow-up, or description provided of those lost *
c) follow up rate $<80 \%$ and no description of those lost to follow-up
d) no statement 


\section{8 | Chapter 5}

Table S5.1. Classification of cognitive domains and included tests ${ }^{A}$

\begin{tabular}{ll}
\hline Cognitive domain & Included tests \\
\hline Global score & Mini mental state examination \\
& Cognitive efficiency profile \\
& Blessed information-memory-concentration test \\
\hline Memory & Auditory verbal learning test \\
& California verbal learning test \\
& Benton visual retention test \\
& Digit span forward (item of Wechsler adult intelligence scale) \\
& Logical memory-delayed (item of Wechsler Memory Scale) \\
& Hopkins verbal learning test \\
\hline Processing speed & Letter digit substitution test (item of Wechsler adult intelligence scale) \\
& Stroop test part I and II \\
& Trail making test-part A \\
\hline Executive function and attention & Verbal fluency test \\
& Word fluency test \\
& Category fluency test \\
& Trail making test-part B \\
& Stroop test part III \\
& Digit span backward (item of Wechsler adult intelligence scale) \\
& Clock drawing test \\
\hline
\end{tabular}

A For tests on domain-specific cognitive function, (composite) scores per domain were extracted as classified in the original study. If classifications were not reported, tests were classified as described in the table. 


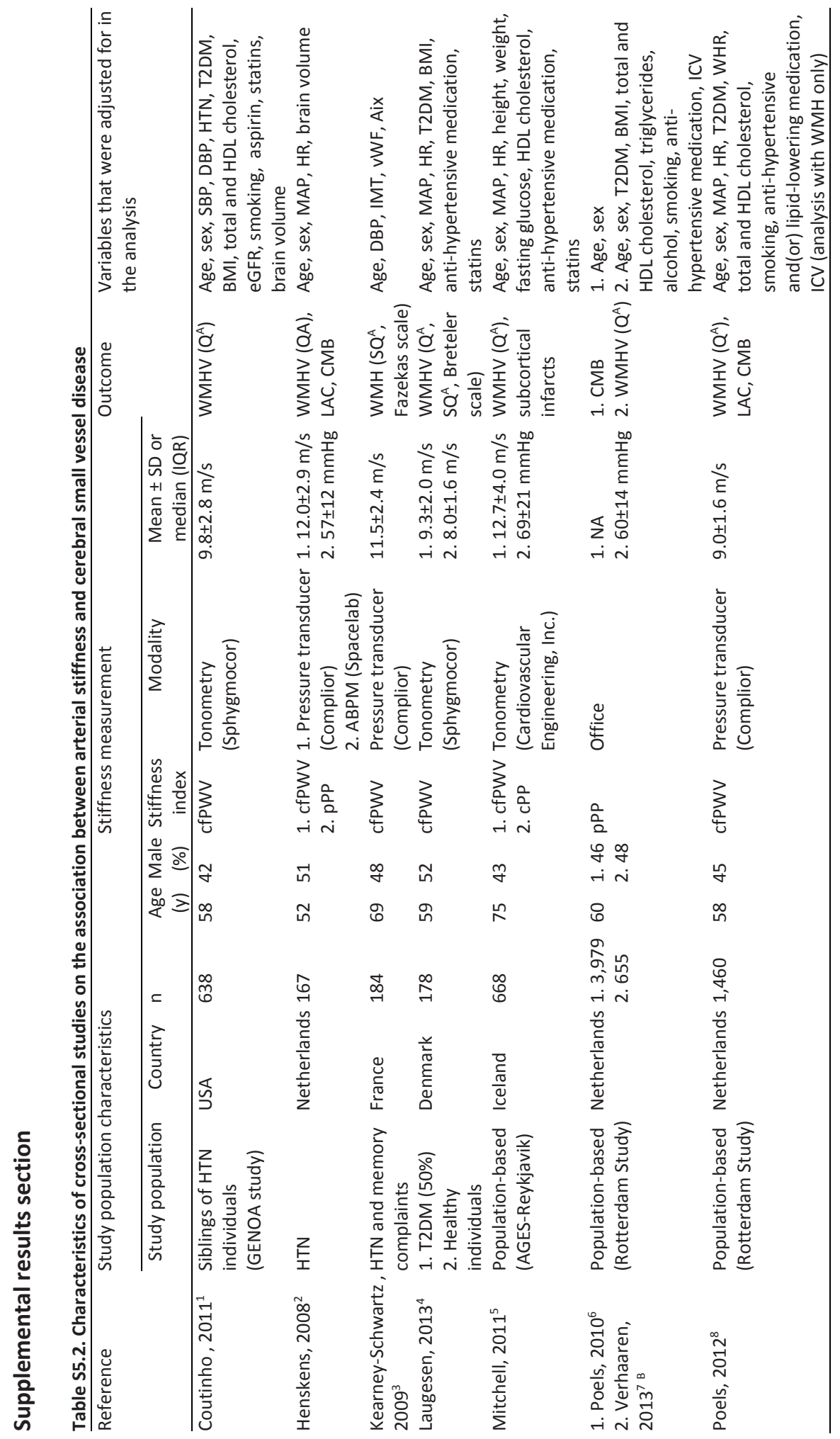




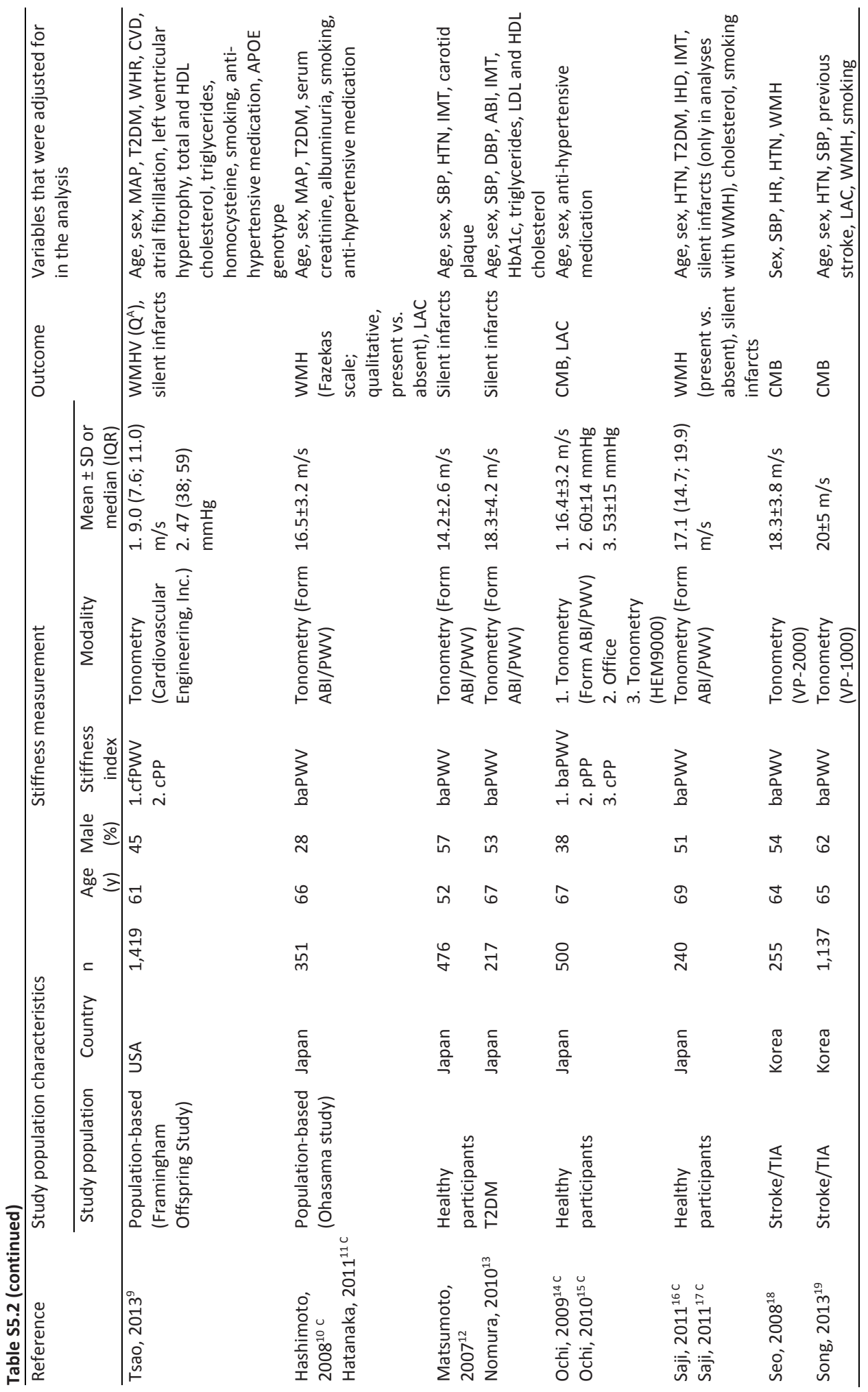




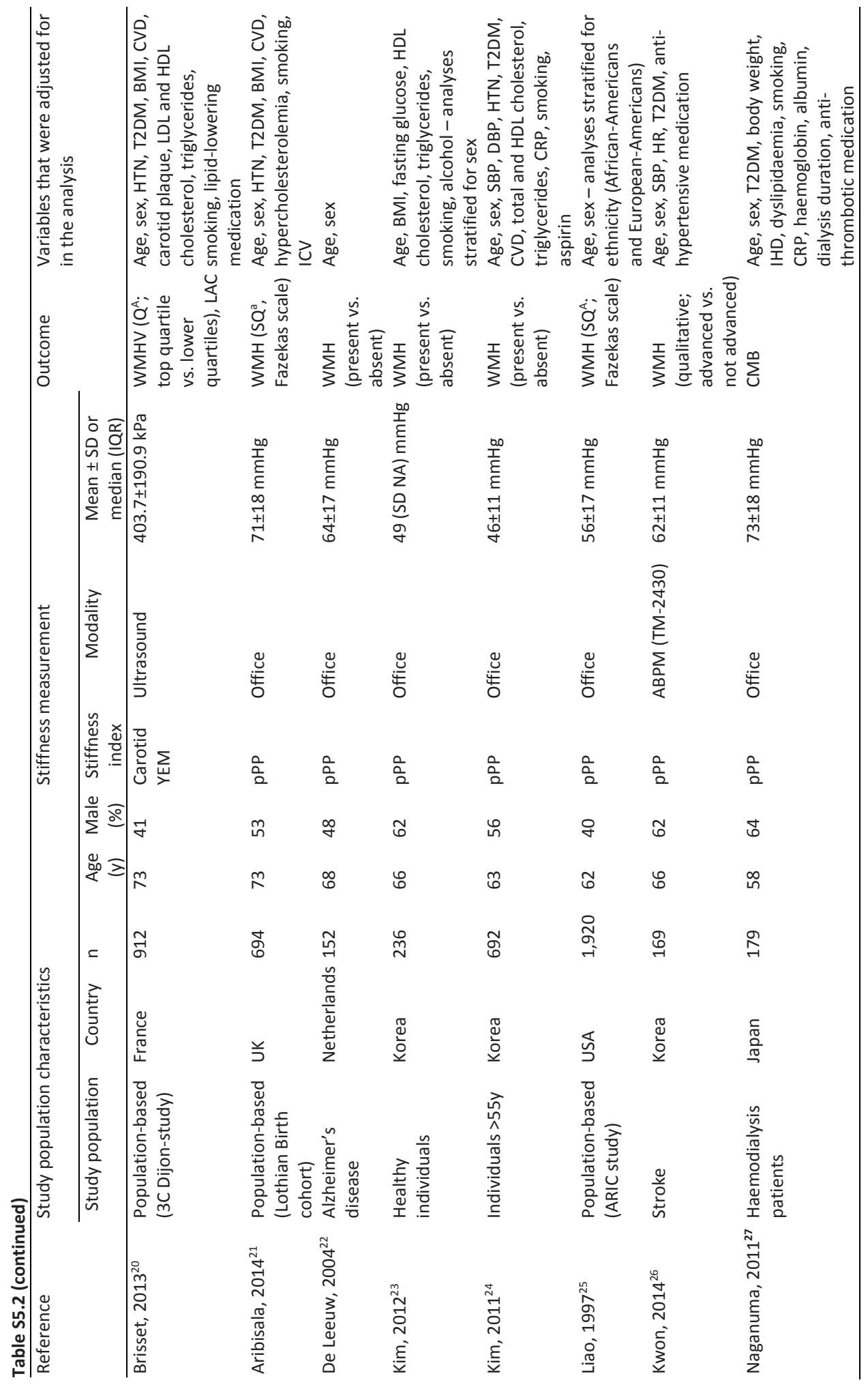




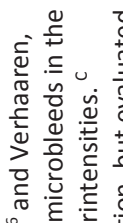

을 흘

ริ)

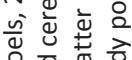

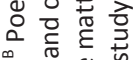

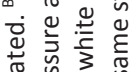

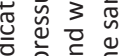

.둘

เม

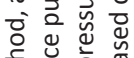

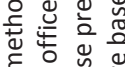

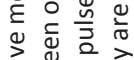

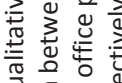

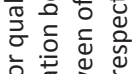

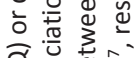

흔 흘

ข้ำ

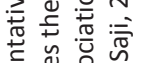

部影尊

站彭

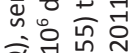

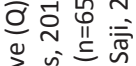

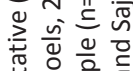

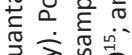

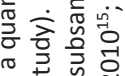

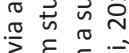

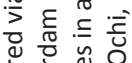

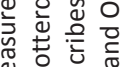

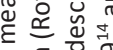

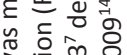

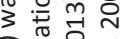

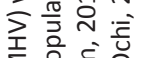

翌

站

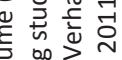

烈

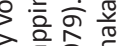

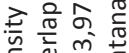

보을

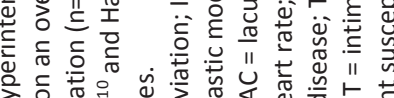

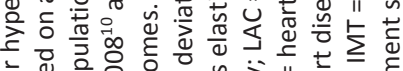

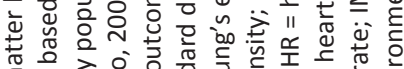

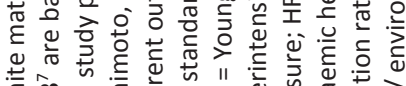

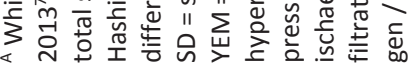

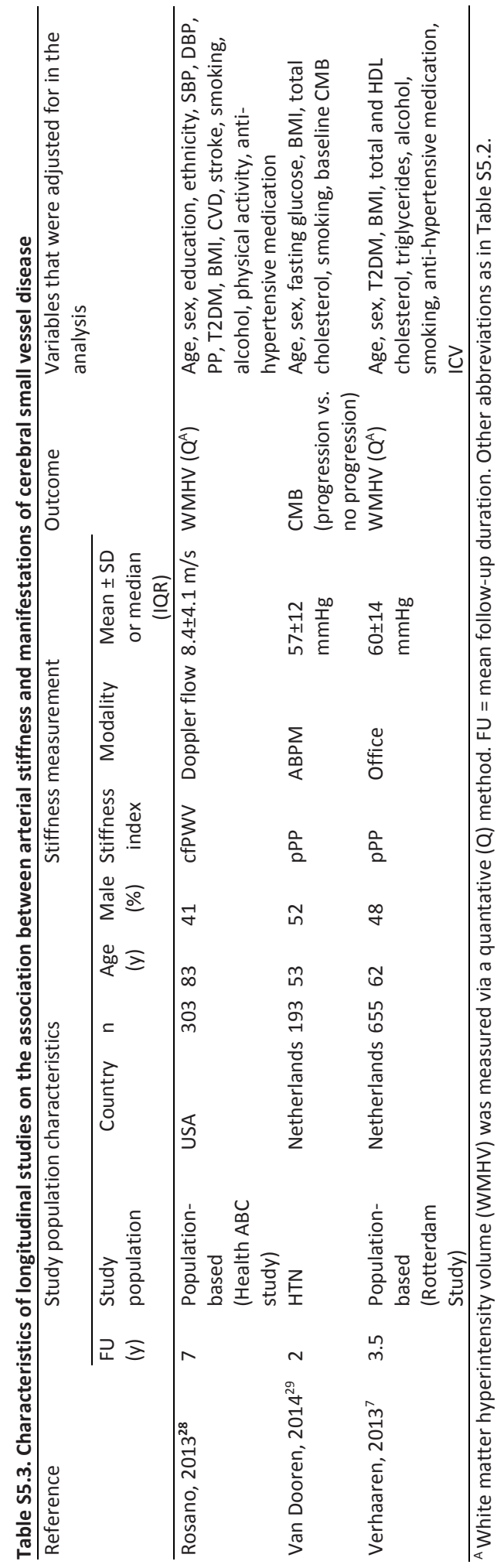




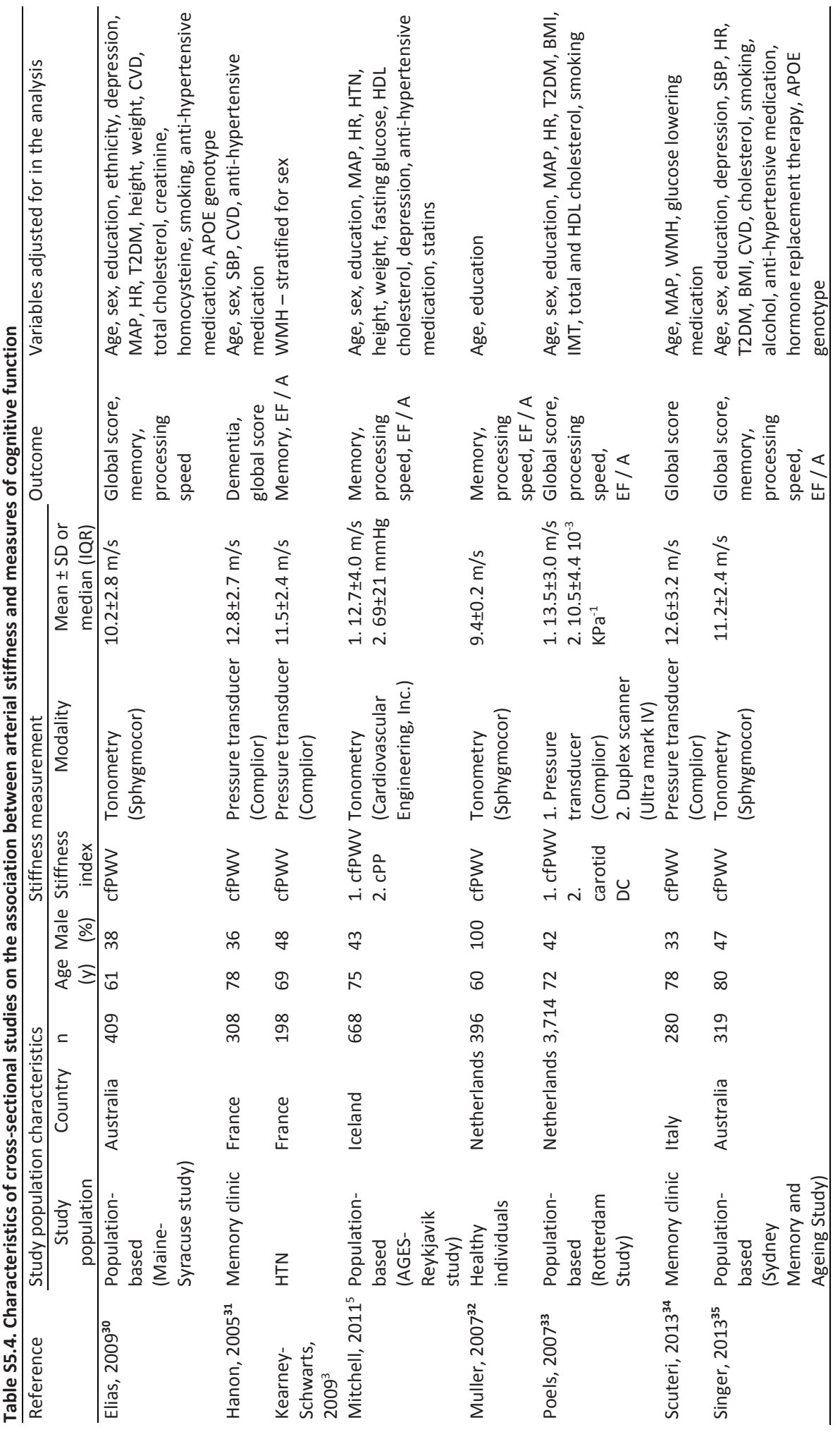




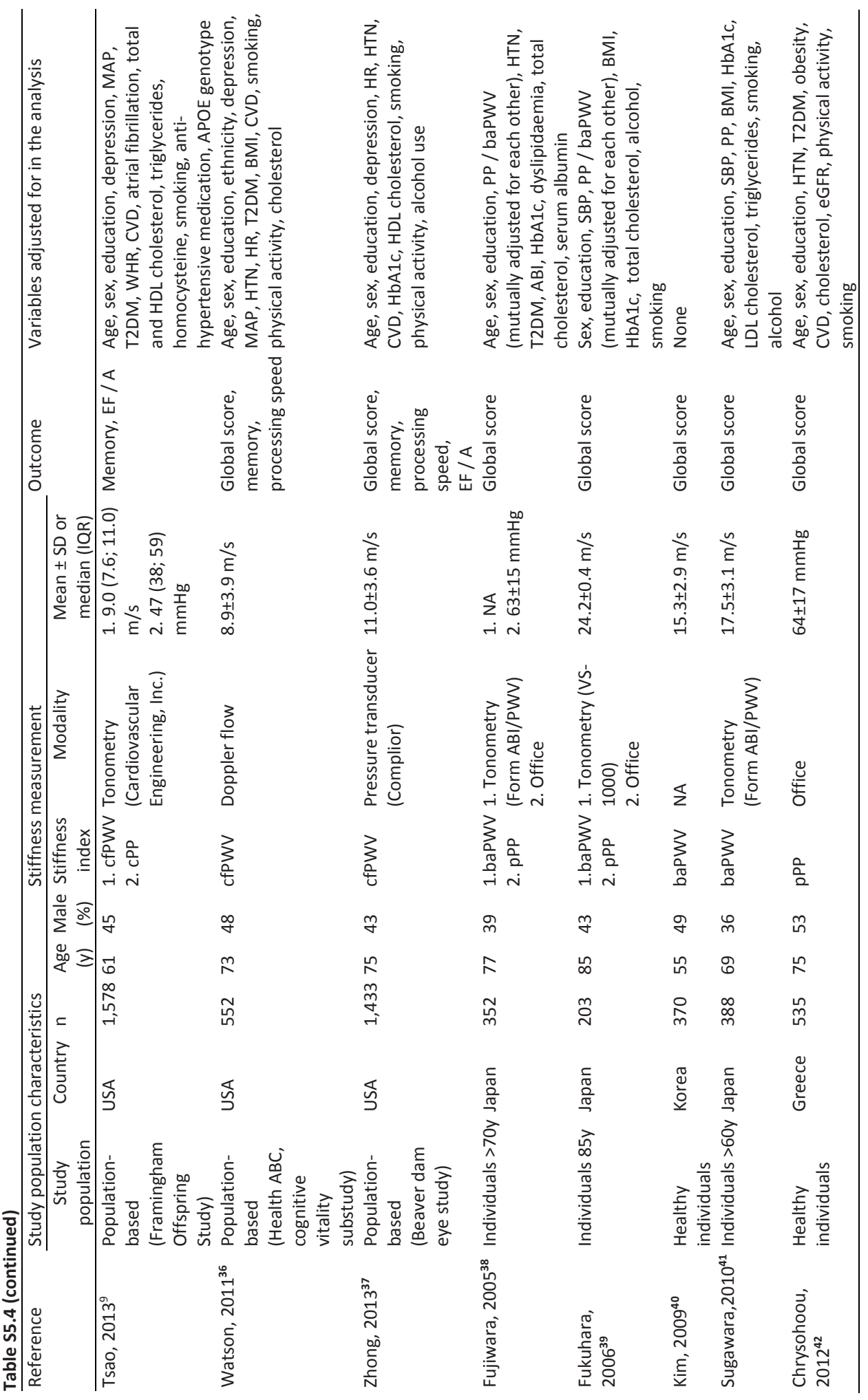




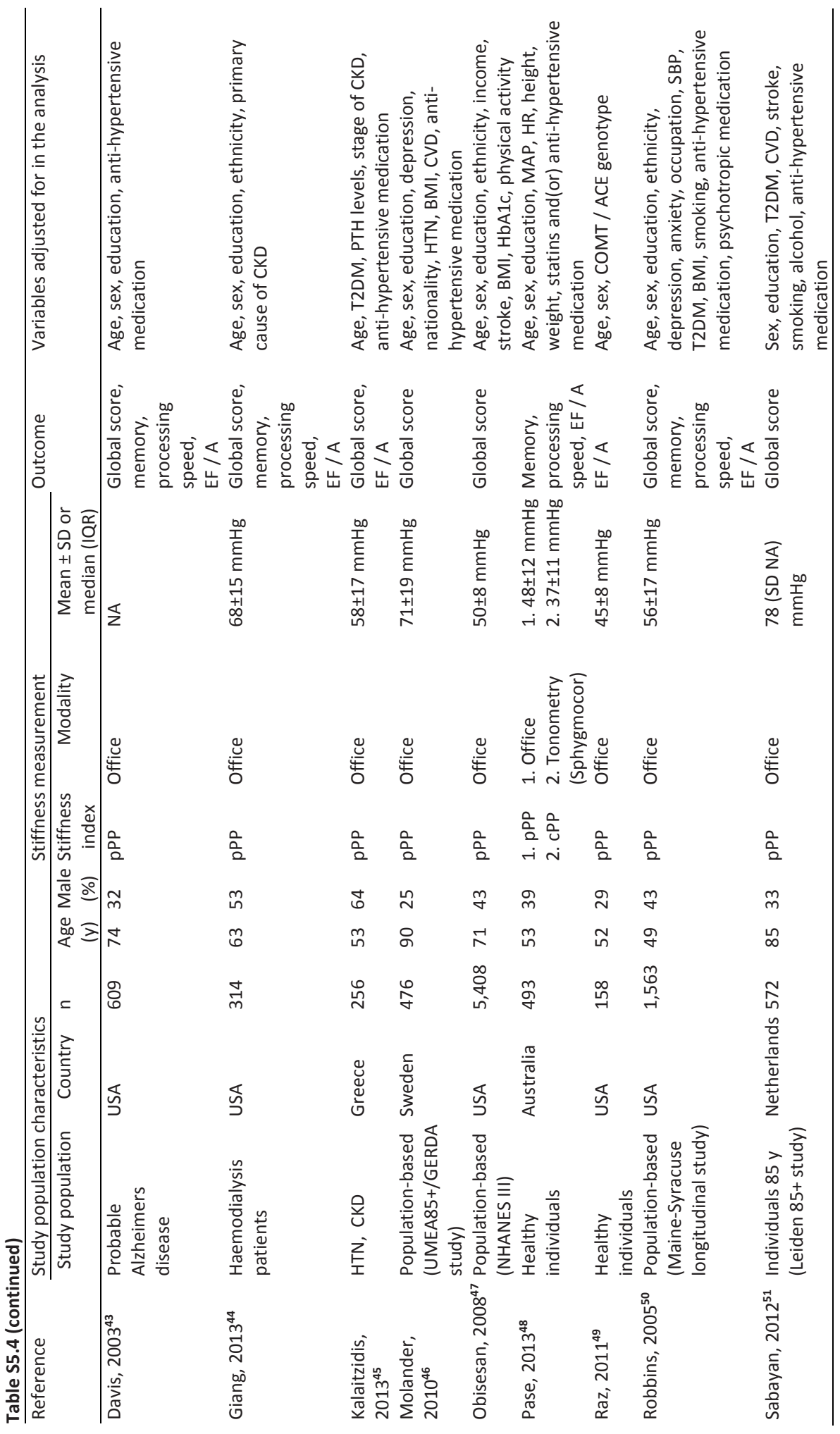




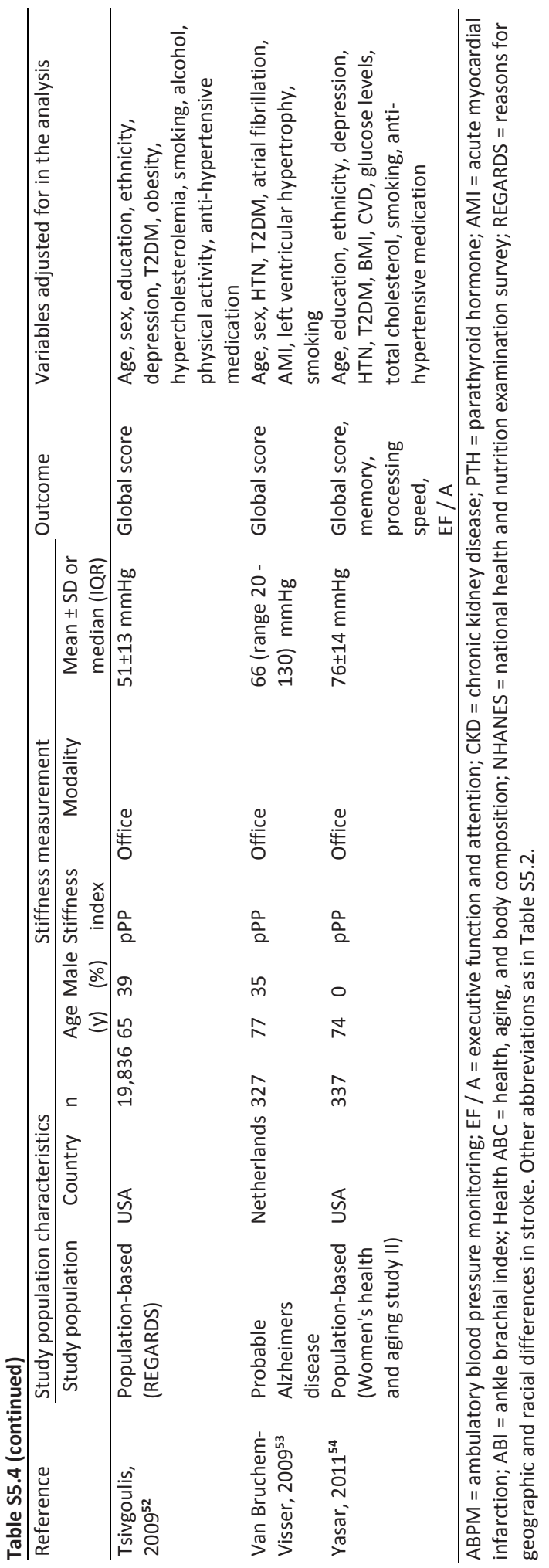




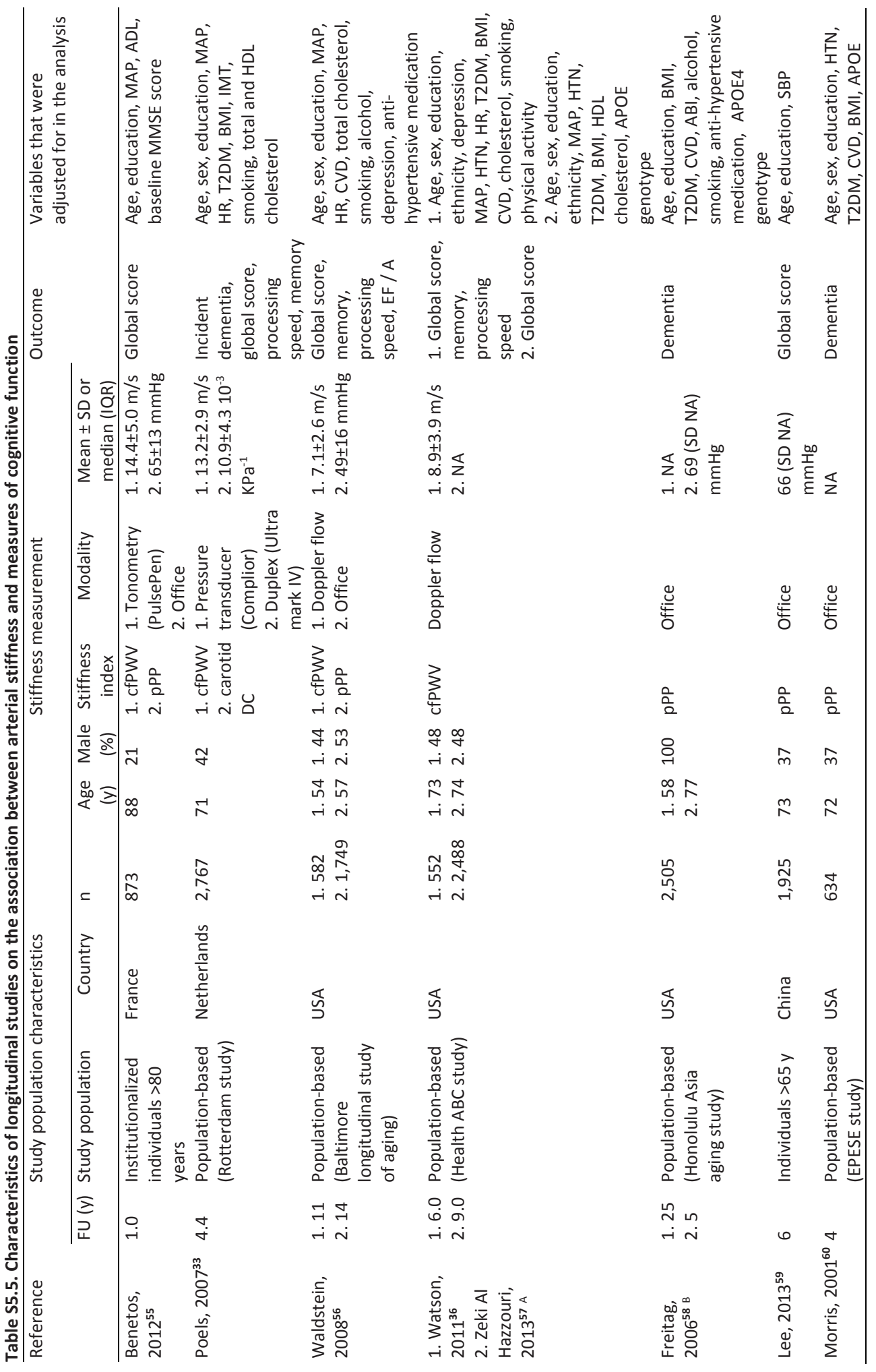




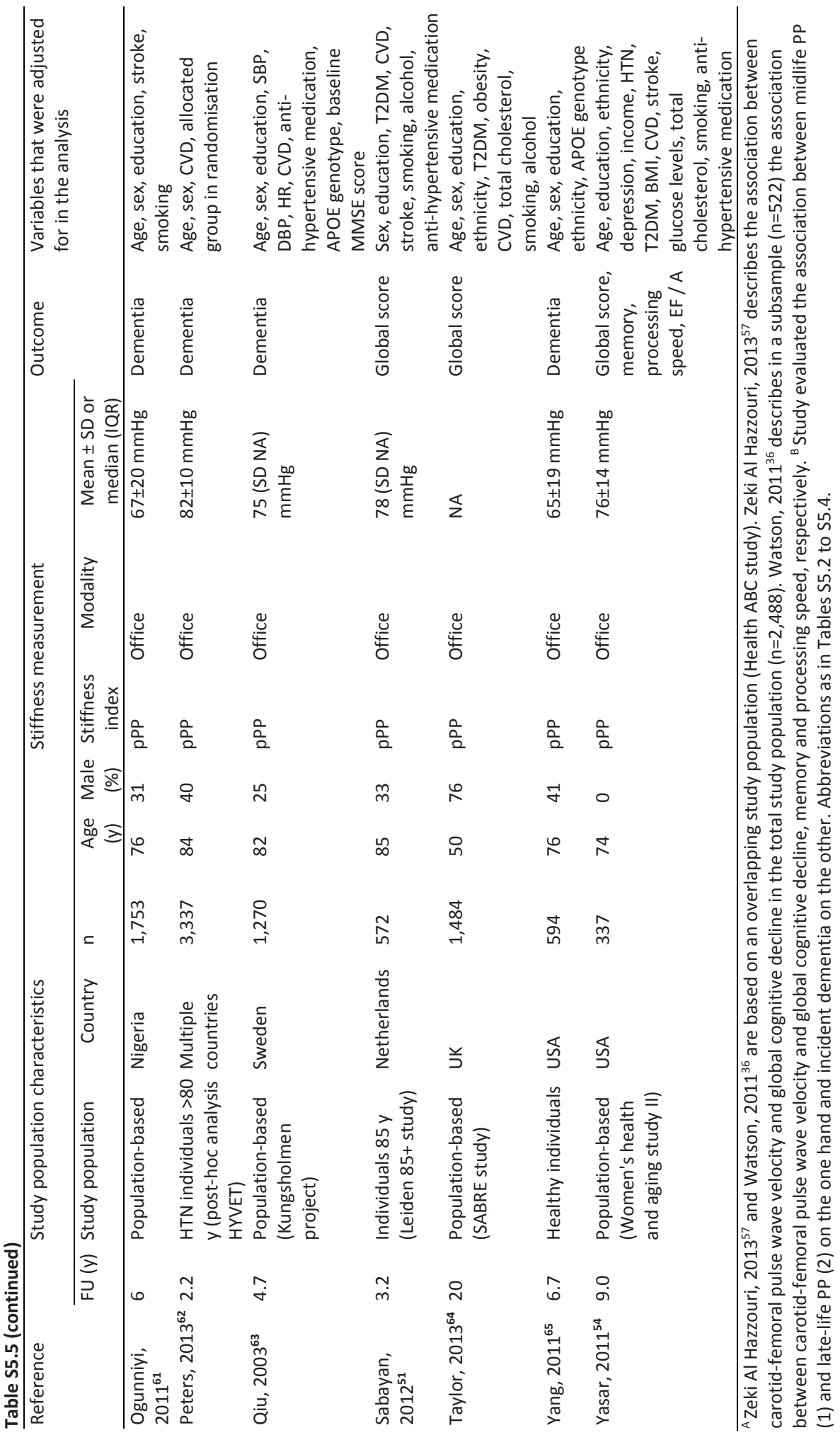


Table S5.6. Newcastle-Ottowa Scale scores for studies on the association between arterial stiffness on the one hand and manifestations of cerebral small vessel disease and measures of cognitive function on the other ${ }^{A}$

\begin{tabular}{|c|c|c|c|c|c|c|c|c|c|c|}
\hline Reference & Design & S1 & $\mathrm{S} 2$ & S3 & C1a & C1b & 01 & $\mathrm{O} 2$ & $\mathrm{O3}$ & $\begin{array}{l}\text { Total } \\
\text { score }\end{array}$ \\
\hline \multicolumn{11}{|l|}{ Cerebral small vessel disease } \\
\hline \multicolumn{11}{|c|}{ Carotid-femoral pulse wave velocity } \\
\hline Coutinho, $2011^{1}$ & CS & 0 & 1 & $\ldots$ & 1 & 1 & 1 & $\ldots$ & $\ldots$ & 4 \\
\hline Henskens, $2008^{2}$ & CS & 0 & 1 & $\ldots$ & 1 & 1 & 1 & $\ldots$ & $\ldots$ & 4 \\
\hline Kearney-Schwartz, $2009^{3}$ & CS & 0 & 1 & $\ldots$ & 1 & 0 & 0 & $\ldots$ & $\ldots$ & 2 \\
\hline Laugesen, $2013^{4}$ & CS & 0 & 1 & $\ldots$ & 1 & 1 & 1 & $\ldots$ & $\ldots$ & 4 \\
\hline Mitchelll, $2011^{5}$ & CS & 1 & 1 & $\ldots$ & 1 & 1 & 1 & $\ldots$ & $\ldots$ & 5 \\
\hline Poels, $2012^{8}$ & $\mathrm{CS}$ & 1 & 1 & $\ldots$ & 1 & 1 & 1 & $\ldots$ & $\ldots$ & 5 \\
\hline Tsao, $2013^{9}$ & CS & 1 & 1 & $\ldots$ & 1 & 1 & 1 & $\ldots$ & $\ldots$ & 5 \\
\hline Rosano, $2013^{28}$ & $\mathrm{~L}$ & 1 & 0 & 0 & 1 & 1 & 1 & 1 & 0 & 5 \\
\hline \multicolumn{11}{|l|}{ Brachial-ankle pulse wave velocity } \\
\hline $\begin{array}{l}\text { Hashimoto, } 2008^{10} / \text { Hatanaka, } \\
2011^{11 \text { B }}\end{array}$ & CS & 1 & 1 & $\ldots$ & 1 & 1 & 0 & $\ldots$ & $\ldots$ & 4 \\
\hline Matsumoto, $2007^{12}$ & CS & 0 & 1 & $\ldots$ & 1 & 1 & 1 & $\ldots$ & $\ldots$ & 4 \\
\hline Nomura, $2010^{13}$ & CS & 0 & 1 & $\ldots$ & 1 & 1 & 1 & $\ldots$ & $\ldots$ & 4 \\
\hline Ochi, $2009^{14}$ / Ochi, $2010^{15 ~ в ~}$ & CS & 1 & 1 & $\ldots$ & 0 & 1 & 1 & $\ldots$ & $\ldots$ & 4 \\
\hline Saji, $2011^{16} /$ Saji, $2012^{17 \text { B }}$ & CS & 0 & 1 & $\ldots$ & 0 & 1 & 1 & $\ldots$ & $\ldots$ & 3 \\
\hline Seo, $2008^{18}$ & $\mathrm{CS}$ & 0 & 1 & $\ldots$ & 1 & 1 & 1 & $\ldots$ & $\ldots$ & 4 \\
\hline Song, $2013^{19}$ & $\mathrm{CS}$ & 0 & 1 & $\ldots$ & 1 & 1 & 1 & $\ldots$ & $\ldots$ & 4 \\
\hline \multicolumn{11}{|l|}{ Local carotid stiffness } \\
\hline Brisset, $2013^{20}$ & CS & 1 & 1 & $\ldots$ & 0 & 1 & 1 & $\ldots$ & $\ldots$ & 4 \\
\hline \multicolumn{11}{|l|}{ Brachial pulse pressure } \\
\hline Aribisala, $2014^{21}$ & CS & 1 & 1 & $\ldots$ & 1 & 0 & 1 & $\ldots$ & $\ldots$ & 4 \\
\hline De Leeuw, $2004^{22}$ & $\mathrm{CS}$ & 0 & 1 & $\ldots$ & 0 & 1 & 0 & $\ldots$ & $\ldots$ & 2 \\
\hline Kim, $2012^{23}$ & CS & 1 & 1 & $\ldots$ & 0 & 1 & 1 & $\ldots$ & $\ldots$ & 4 \\
\hline Kim, $2011^{24}$ & CS & 1 & 1 & $\ldots$ & 1 & 1 & 1 & $\ldots$ & $\ldots$ & 5 \\
\hline Kwon, $2014^{26}$ & CS & 0 & 1 & $\ldots$ & 1 & 1 & 1 & $\ldots$ & $\ldots$ & 4 \\
\hline Liao, $1997^{25}$ & CS & 1 & 1 & $\ldots$ & 0 & 1 & 1 & $\ldots$ & $\ldots$ & 4 \\
\hline Naganuma, $2011^{27}$ & CS & 0 & 0 & $\ldots$ & 1 & 0 & 1 & $\ldots$ & $\ldots$ & 2 \\
\hline Ochi, $2010^{15}$ & CS & 1 & 1 & $\ldots$ & 0 & 1 & 1 & $\ldots$ & $\ldots$ & 4 \\
\hline Poels, $2010^{6}$ & CS & 1 & 1 & $\ldots$ & 0 & 1 & 1 & $\ldots$ & $\ldots$ & 4 \\
\hline Verhaaren, $2013^{7}$ & $\mathrm{CS}$ & 1 & 1 & $\ldots$ & 0 & 1 & 1 & $\ldots$ & $\ldots$ & 4 \\
\hline Henskens, $2008^{2}$ & CS & 0 & 1 & $\ldots$ & 1 & 1 & 1 & $\ldots$ & $\ldots$ & 4 \\
\hline Van Dooren, $2014^{29}$ & $\mathrm{~L}$ & 0 & 1 & 1 & 1 & 0 & 1 & 0 & 1 & 5 \\
\hline Verhaaren, $2013^{7}$ & L & 1 & 1 & 1 & 0 & 1 & 1 & 0 & 1 & 6 \\
\hline \multicolumn{11}{|l|}{ Central pulse pressure } \\
\hline Mitchell, $2011^{5}$ & CS & 1 & 1 & $\ldots$ & 1 & 1 & 1 & $\ldots$ & $\ldots$ & 5 \\
\hline Ochi, $2010^{15}$ & CS & 1 & 1 & $\ldots$ & 0 & 1 & 1 & $\ldots$ & $\ldots$ & 4 \\
\hline Tsao, $2013^{9}$ & CS & 1 & 1 & $\ldots$ & 1 & 1 & 1 & $\ldots$ & $\ldots$ & 5 \\
\hline \multicolumn{11}{|c|}{ Cognitive function } \\
\hline \multicolumn{11}{|c|}{ Carotid-femoral pulse wave velocity } \\
\hline Elias, $2009^{30}$ & CS & 1 & 1 & $\ldots$ & 1 & 1 & 1 & $\ldots$ & $\ldots$ & 5 \\
\hline Hanon, $2005^{31}$ & CS & 0 & 1 & $\ldots$ & 1 & 0 & 1 & $\ldots$ & $\ldots$ & 3 \\
\hline Kearney-Schwartz, $2009^{3}$ & $\mathrm{CS}$ & 0 & 1 & $\ldots$ & 0 & 0 & 1 & $\ldots$ & $\ldots$ & 2 \\
\hline Mitchell, $2007^{5}$ & CS & 1 & 1 & $\ldots$ & 1 & 1 & 1 & $\ldots$ & $\ldots$ & 5 \\
\hline Muller, $2007^{32}$ & CS & 1 & 1 & $\ldots$ & 0 & 1 & 1 & $\ldots$ & $\ldots$ & 4 \\
\hline Poels, $2007^{33}$ & $\mathrm{CS}$ & 1 & 1 & $\ldots$ & 1 & 1 & 1 & $\ldots$ & $\ldots$ & 5 \\
\hline Scuteri, $2013^{34}$ & $\mathrm{CS}$ & 0 & 1 & $\ldots$ & 1 & 0 & 1 & $\ldots$ & $\ldots$ & 3 \\
\hline Singer, $2013^{35}$ & CS & 1 & 1 & $\ldots$ & 1 & 1 & 1 & $\ldots$ & $\ldots$ & 5 \\
\hline Tsao, $2013^{9}$ & CS & 1 & 1 & $\ldots$ & 1 & 1 & 1 & $\ldots$ & $\ldots$ & 5 \\
\hline Watson, $2011^{36}$ & CS & 1 & 0 & $\ldots$ & 1 & 1 & 1 & $\ldots$ & $\ldots$ & 4 \\
\hline Zhong, $2013^{37}$ & $\mathrm{CS}$ & 1 & 1 & $\ldots$ & 0 & 1 & 1 & $\ldots$ & $\ldots$ & 4 \\
\hline Benetos, $2012^{55}$ & $\mathrm{~L}$ & 0 & 1 & 1 & 1 & 1 & 1 & 0 & 1 & 6 \\
\hline
\end{tabular}


Table S5.6 (continued)

\begin{tabular}{|c|c|c|c|c|c|c|c|c|c|c|}
\hline Reference & Design & S1 & $\mathrm{S} 2$ & S3 & C1a & $\mathrm{C} 1 \mathrm{~b}$ & 01 & $\mathrm{O} 2$ & $\mathrm{O3}$ & $\begin{array}{l}\text { Total } \\
\text { score }\end{array}$ \\
\hline Poels, $2007^{33}$ & $\mathrm{~L}$ & 1 & 1 & 1 & 1 & 1 & 1 & 1 & 1 & 8 \\
\hline Waldstein, $2008^{56}$ & $\mathrm{~L}$ & 1 & 0 & 1 & 1 & 1 & 1 & 1 & 0 & 6 \\
\hline Watson, $2011^{36}$ & $\mathrm{~L}$ & 1 & 0 & 0 & 1 & 1 & 1 & 1 & 0 & 5 \\
\hline Zeki Al Hazzouri, 2013 ${ }^{57}$ & $\mathrm{~L}$ & 1 & 0 & 1 & 1 & 1 & 1 & 1 & 0 & 6 \\
\hline \multicolumn{11}{|c|}{ Brachial-ankle pulse wave velocity } \\
\hline Fujiwara, $2005^{38}$ & CS & 0 & 1 & $\ldots$ & 1 & 1 & 1 & $\ldots$ & $\ldots$ & 4 \\
\hline Fukuhara, $2006^{39}$ & CS & 0 & 1 & $\ldots$ & 1 & 1 & 1 & $\ldots$ & $\ldots$ & 4 \\
\hline Kim, $2009^{40}$ & CS & 1 & 0 & $\ldots$ & 0 & 0 & 1 & $\ldots$ & $\ldots$ & 2 \\
\hline Suguwara, $2010^{41}$ & CS & 1 & 1 & $\ldots$ & 1 & 1 & 1 & $\ldots$ & $\ldots$ & 5 \\
\hline \multicolumn{11}{|l|}{ Local carotid stiffness } \\
\hline Poels, $2007^{33}$ & CS & 1 & 1 & $\ldots$ & 1 & 1 & 1 & $\ldots$ & $\ldots$ & 5 \\
\hline Poels, $2007^{33}$ & $\mathrm{~L}$ & 1 & 1 & 1 & 1 & 1 & 1 & 1 & 1 & 8 \\
\hline \multicolumn{11}{|l|}{ Brachial pulse pressure } \\
\hline Chrysohoou, 2012 & $\mathrm{CS}$ & 1 & 0 & $\ldots$ & 0 & 1 & 1 & $\ldots$ & $\ldots$ & 3 \\
\hline Davis, $2003^{43}$ & CS & 0 & 1 & $\ldots$ & 0 & 1 & 1 & $\ldots$ & $\ldots$ & 3 \\
\hline Fujiwara, $2005^{38}$ & CS & 1 & 0 & $\ldots$ & 0 & 1 & 1 & $\ldots$ & $\ldots$ & 3 \\
\hline Fukuhara, $2006^{39}$ & CS & 1 & 0 & $\ldots$ & 1 & 1 & 1 & $\ldots$ & $\ldots$ & 4 \\
\hline Giang, $2013^{44}$ & $\mathrm{CS}$ & 0 & 0 & $\ldots$ & 0 & 1 & 1 & $\ldots$ & $\ldots$ & 2 \\
\hline Kalaitzidis, $2013^{45}$ & CS & 0 & 0 & $\ldots$ & 0 & 0 & 1 & $\ldots$ & $\ldots$ & 1 \\
\hline Molander, $2010^{46}$ & $\mathrm{CS}$ & 1 & 1 & $\ldots$ & 0 & 1 & 1 & $\ldots$ & $\ldots$ & 4 \\
\hline Obisesan, $2008^{47}$ & CS & 1 & 1 & $\ldots$ & 0 & 1 & 1 & $\ldots$ & $\ldots$ & 4 \\
\hline Pase, $2013^{48}$ & $\mathrm{CS}$ & 1 & 1 & $\ldots$ & 1 & 1 & 0 & $\ldots$ & $\ldots$ & 4 \\
\hline Raz, $2011^{49}$ & $\mathrm{CS}$ & 1 & 1 & $\ldots$ & 0 & 0 & 1 & $\ldots$ & $\ldots$ & 3 \\
\hline Robbins, $2005^{50}$ & $\mathrm{CS}$ & 1 & 1 & $\ldots$ & 1 & 1 & 1 & $\ldots$ & $\ldots$ & 5 \\
\hline Sabayan, $2012^{51}$ & $\mathrm{CS}$ & 1 & 1 & $\ldots$ & 0 & 1 & 1 & $\ldots$ & $\ldots$ & 4 \\
\hline Tsivgoulis, $2009^{52}$ & $\mathrm{CS}$ & 1 & 1 & $\ldots$ & 0 & 1 & 1 & $\ldots$ & $\ldots$ & 4 \\
\hline Van Bruchem-Visser, $2009^{53}$ & CS & 0 & 1 & $\ldots$ & 0 & 0 & 1 & $\ldots$ & $\ldots$ & 2 \\
\hline Yasar, $2011^{54}$ & CS & 1 & 1 & $\ldots$ & 0 & 1 & 1 & $\ldots$ & $\ldots$ & 4 \\
\hline Benetos, $2012^{55}$ & $\mathrm{~L}$ & 0 & 1 & 1 & 1 & 1 & 1 & 0 & 1 & 6 \\
\hline Freitag, $2006^{58}$ & $\mathrm{~L}$ & 1 & 1 & 1 & 0 & 1 & 1 & 1 & 1 & 7 \\
\hline Lee, $2013^{59}$ & $\mathrm{~L}$ & 1 & 1 & 1 & 1 & 1 & 1 & 1 & 0 & 7 \\
\hline Morris, $2001^{60}$ & $\mathrm{~L}$ & 1 & 1 & 1 & 0 & 1 & 1 & 1 & 1 & 7 \\
\hline Ogunniyi, $2011^{61}$ & $\mathrm{~L}$ & 1 & 1 & 1 & 0 & 1 & 1 & 1 & 1 & 7 \\
\hline Peters, $2013^{62}$ & $\mathrm{~L}$ & 0 & 1 & 1 & 0 & 0 & 1 & 1 & 0 & 4 \\
\hline Qiu, $2013^{63}$ & $\mathrm{~L}$ & 1 & 1 & 1 & 1 & 1 & 1 & 1 & 1 & 8 \\
\hline Sabayan, $2012^{51}$ & $L$ & 1 & 1 & 1 & 0 & 1 & 1 & 0 & 1 & 6 \\
\hline Taylor, $2013^{64}$ & $\mathrm{~L}$ & 1 & 1 & 0 & 0 & 1 & 1 & 1 & 1 & 6 \\
\hline Waldstein, $2008^{56}$ & $\mathrm{~L}$ & 1 & 1 & 1 & 1 & 1 & 1 & 1 & 0 & 7 \\
\hline Yang, $2011^{65}$ & $\mathrm{~L}$ & 0 & 1 & 1 & 0 & 1 & 1 & 1 & 0 & 5 \\
\hline Yasar, $2011^{54}$ & $\mathrm{~L}$ & 1 & 1 & 1 & 0 & 1 & 1 & 1 & 1 & 7 \\
\hline \multicolumn{11}{|l|}{ Central pulse pressure } \\
\hline Mitchell, $2011^{5}$ & $\mathrm{CS}$ & 1 & 1 & $\ldots$ & 1 & 1 & 1 & $\ldots$ & $\ldots$ & 5 \\
\hline Pase, $2013^{48}$ & CS & 1 & 1 & $\ldots$ & 1 & 1 & 0 & $\ldots$ & $\ldots$ & 4 \\
\hline Tsao, $2013^{9}$ & $\mathrm{CS}$ & 1 & 1 & $\ldots$ & 1 & 1 & 1 & $\ldots$ & $\ldots$ & 5 \\
\hline
\end{tabular}

A For an explanation of the individual items, see the provided Newcastle-Ottowa Scale. Maximal NOS score for a longitudinal study is 8 and for a cross-sectional study 5. ${ }^{B}$ Hashimoto, $2008^{10}$ and Hatanaka, 2011 ${ }^{11}$; Ochi, $2009^{14}$ and Ochi, 2010 ${ }^{15}$; and Saji, $2011^{16}$ and Saji, $2012^{17}$, respectively are based on the same study population. $\mathrm{S}=$ selection, $\mathrm{C}=$ comparability, $\mathrm{O}=$ outcome, $\mathrm{CS}=$ cross-sectional, $\mathrm{L}=$ longitudinal. 
Arterial stiffness, cerebral small vessel disease and cognitive impairment | 131

A

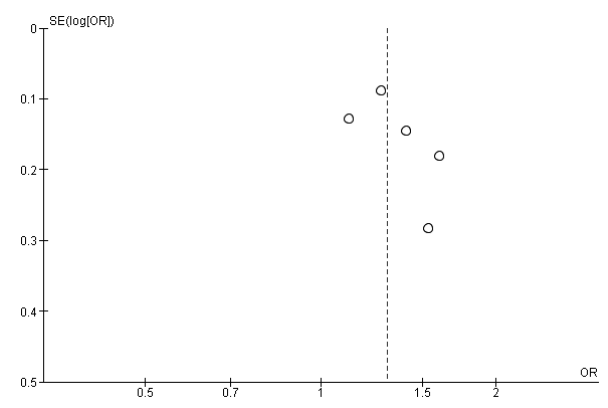

C

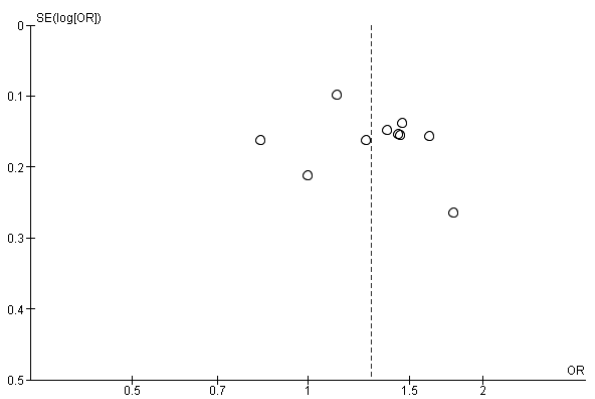

B

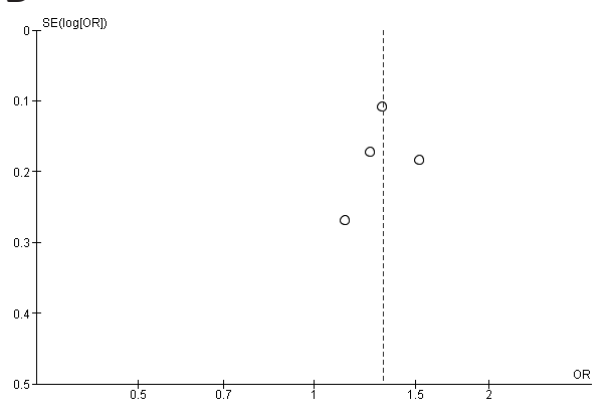

Figure S5.1. Funnel plot for the pooled association between arterial stiffness and white matter hyperintensities (panel A), cerebral microbleeds (panel B) and cerebral infarcts (panel C). Funnel plot indicate odds ratios (OR) against the standard error (SE) of each study. 
Study reference $n$ Age Odds ratio (95\% confidence interval)

A. Carotid-femoral pulse wave velocity (per SD)

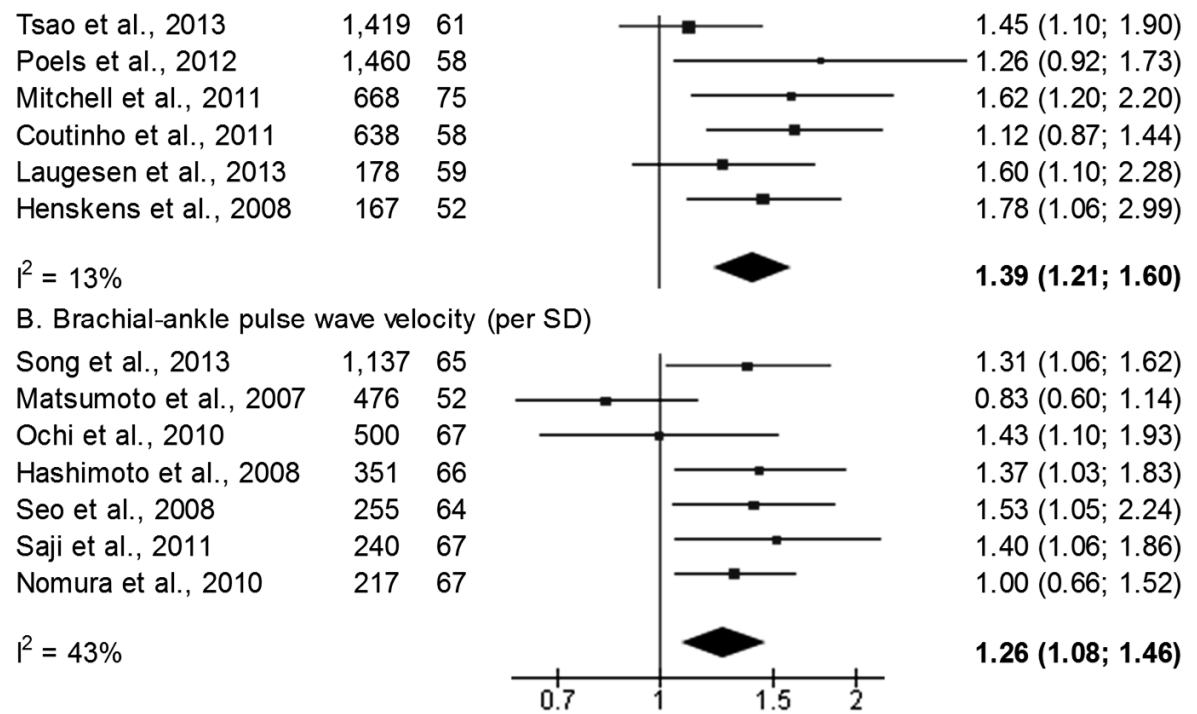

Figure S5.2. Association between carotid-femoral pulse wave velocity (panel A) and brachial-ankle pulse wave velocity (panel B) on the one hand and manifestations of cerebral small vessel disease on the other. For these analyses, the results for different manifestations of cerebral small vessel disease were pooled together. When multiple manifestations were evaluated in the same study population, only the manifestation was included with the highest prevalence. Included studies for carotid-femoral pulse wave velocity evaluated the association with

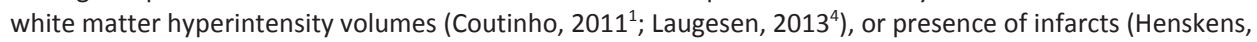
2008'; Mitchell, 2011 ${ }^{5}$; Poels, $2012^{8}$; Tsao, $2013^{9}$ ). Included studies for brachial-ankle pulse wave velocity evaluated the association with white matter hyperintensities (Saji, 2012 ${ }^{16}$ ), microbleeds (Seo, 2008 ${ }^{18}$; Song, $2013^{19}$ ), or infarcts (Hashimoto, 2008 $8^{10}$; Matsumoto, 2007 ${ }^{12}$; Ochi, 2010 ${ }^{15}$; Nomura, $2007^{13}$ ).

\section{References}

1. Coutinho T, Turner ST, Kullo IJ. Aortic pulse wave velocity is associated with measures of subclinical target organ damage. JACC. Cardiovascular imaging. 2011;4:754-761.

2. Henskens LH, Kroon AA, van Oostenbrugge RJ, et al. Increased aortic pulse wave velocity is associated with silent cerebral small-vessel disease in hypertensive patients. Hypertension. 2008;52:1120-1126.

3. Kearney-Schwartz A, Rossignol P, Bracard S, et al. Vascular structure and function is correlated to cognitive performance and white matter hyperintensities in older hypertensive patients with subjective memory complaints. Stroke. 2009;40:1229-1236

4. Laugesen E, Hoyem P, Stausbol-Gron B, et al. Carotid-femoral pulse wave velocity is associated with cerebral white matter lesions in type 2 diabetes. Diabetes Care. 2013;36:722-728

5. Mitchell GF, van Buchem MA, Sigurdsson S, et al. Arterial stiffness, pressure and flow pulsatility and brain structure and function: the Age, Gene/Environment Susceptibility--Reykjavik study. Brain. 2011;134:3398-3407.

6. Poels MM, Vernooij MW, Ikram MA, et al. Prevalence and risk factors of cerebral microbleeds: an update of the Rotterdam scan study. Stroke. 2010;41:S103-106.

7. Verhaaren $\mathrm{BF}$, Vernooij MW, de Boer R, et al. High blood pressure and cerebral white matter lesion progression in the general population. Hypertension. 2013;61:1354-1359. 
8. Poels MM, Zaccai K, Verwoert GC, et al. Arterial stiffness and cerebral small vessel disease: the Rotterdam Scan Study. Stroke. 2012;43:2637-2642.

9. Tsao CW, Seshadri S, Beiser AS, et al. Relations of arterial stiffness and endothelial function to brain aging in the community. Neurology. 2013.

10. Hashimoto J, Aikawa T, Imai Y. Large artery stiffening as a link between cerebral lacunar infarction and renal albuminuria. Am J Hypertens. 2008;21:1304-1309.

11. Hatanaka R, Obara T, Watabe D, et al. Association of arterial stiffness with silent cerebrovascular lesions: the Ohasama study. Cerebrovasc Dis. 2011;31:329-337.

12. Matsumoto $\mathrm{M}$, Inoue $\mathrm{K}$, Moriki A. Associations of brachial-ankle pulse wave velocity and carotid atherosclerotic lesions with silent cerebral lesions. Hypertens Res. 2007;30:767-773.

13. Nomura K, Hamamoto Y, Takahara S, et al. Relationship between carotid intima-media thickness and silent cerebral infarction in Japanese subjects with type 2 diabetes. Diabetes Care. 2010;33:168-170.

14. Ochi N, Tabara Y, Igase $M$, et al. Silent cerebral microbleeds associated with arterial stiffness in an apparently healthy subject. Hypertens Res. 2009;32:255-260.

15. Ochi N, Kohara K, Tabara Y, et al. Association of central systolic blood pressure with intracerebral small vessel disease in Japanese. Am J Hypertens. 2010;23:889-894.

16. Saji N, Shimizu H, Kawarai T, Tadano M, Kita Y, Yokono K. Increased brachial-ankle pulse wave velocity is independently associated with white matter hyperintensities. Neuroepidemiology. 2011;36:252-257.

17. Saji N, Kimura K, Shimizu H, Kita Y. Association between silent brain infarct and arterial stiffness indicated by brachial-ankle pulse wave velocity. Intern Med. 2012;51:1003-1008.

18. Seo WK, Lee JM, Park MH, Park KW, Lee DH. Cerebral microbleeds are independently associated with arterial stiffness in stroke patients. Cerebrovasc Dis. 2008;26:618-623.

19. Song TJ, Kim J, Kim YD, et al. The distribution of cerebral microbleeds determines their association with arterial stiffness in non-cardioembolic acute stroke patients. Eur J Neurol. 2013 Dec 16 [Epub].

20. Brisset M, Boutouyrie P, Pico F, et al. Large-vessel correlates of cerebral small-vessel disease. Neurology. 2013;80:662-669.

21. Aribisala BS, Morris Z, Eadie E, et al. Blood pressure, internal carotid artery flow parameters, and agerelated white matter hyperintensities. Hypertension. 2014;63:1011-1018.

22. De Leeuw FE, Barkhof F, Scheltens P. Alzheimer's disease--one clinical syndrome, two radiological expressions: a study on blood pressure. J Neurol Neurosurg Psychiatry. 2004;75:1270-1274.

23. Kim SH, Shim JY, Lee HR, Na HY, Lee YJ. The relationship between pulse pressure and leukoaraiosis in the elderly. Arch Gerontol Geriatr. 2012;54:206-209.

24. Kim CK, Lee SH, Kim BJ, Ryu WS, Yoon BW. Age-independent association of pulse pressure with cerebral white matter lesions in asymptomatic elderly individuals. J Hypertens. 2011;29:325-329.

25. Liao D, Cooper L, Cai J, et al. The prevalence and severity of white matter lesions, their relationship with age, ethnicity, gender, and cardiovascular disease risk factors: the ARIC Study. Neuroepidemiology. 1997;16:149-162.

26. Kwon HS, Lim YH, Kim HY, et al. Association of ambulatory blood pressure and heart rate with advanced white matter lesions in ischemic stroke patients. AmJ Hypertension. 2014;27:177-183.

27. Naganuma T, Takemoto Y, Yamasaki T, et al. Factors associated with silent cerebral microbleeds in hemodialysis patients. Clin Nephrol. 2011;75:346-355.

28. Rosano C, Watson N, Chang Y, et al. Aortic pulse wave velocity predicts focal white matter hyperintensities in a biracial cohort of older adults. Hypertension. 2013;61:160-165.

29. van Dooren M, Staals J, de Leeuw PW, Kroon AA, Henskens LH, van Oostenbrugge RJ. Progression of brain microbleeds in essential hypertensive patients: a 2-year follow-up study. Am J Hypertension 2014;27:1045-1051.

30. Elias MF, Robbins MA, Budge MM, Abhayaratna WP, Dore GA, Elias PK. Arterial pulse wave velocity and cognition with advancing age. Hypertension. 2009;53:668-673.

31. Hanon $\mathrm{O}$, Haulon $\mathrm{S}$, Lenoir $\mathrm{H}$, et al. Relationship between arterial stiffness and cognitive function in elderly subjects with complaints of memory loss. Stroke. 2005;36:2193-2197. 
32. Muller M, Grobbee DE, Aleman A, Bots M, van der Schouw YT. Cardiovascular disease and cognitive performance in middle-aged and elderly men. Atherosclerosis. 2007;190:143-149.

33. Poels MM, van Oijen M, Mattace-Raso FU, et al. Arterial stiffness, cognitive decline, and risk of dementia: the Rotterdam study. Stroke. 2007;38:888-892.

34. Scuteri A, Tesauro M, Guglini L, Lauro D, Fini M, Di Daniele N. Aortic stiffness and hypotension episodes are associated with impaired cognitive function in older subjects with subjective complaints of memory loss. Int J Cardiol. 2013;169:371-377.

35. Singer J, Trollor JN, Crawford J, et al. The association between pulse wave velocity and cognitive function: the Sydney Memory and Ageing Study. PloS one. 2013;8:e61855.

36. Watson NL, Sutton-Tyrrell K, Rosano C, et al. Arterial stiffness and cognitive decline in well-functioning older adults. J Gerontol A Biol Sci Med Sci. 2011;66:1336-1342.

37. Zhong W, Cruickshanks KJ, Schubert CR, et al. Pulse Wave Velocity and Cognitive Function in Older Adults. Alzheimer Dis Assoc Disord. 2013 [Epub].

38. Fujiwara Y, Chaves PH, Takahashi R, et al. Arterial pulse wave velocity as a marker of poor cognitive function in an elderly community-dwelling population. J Gerontol A Biol Sci Med Sci. 2005;60:607-612.

39. Fukuhara M, Matsumura K, Ansai T, et al. Prediction of cognitive function by arterial stiffness in the very elderly. Circ J. 2006;70:756-761.

40. Kim YS, Kim DH, Choi BH, Sohn EH, Lee AY. Relationship between brachial-ankle pulse wave velocity and cognitive function in an elderly community-dwelling population with metabolic syndrome. Arch Gerontol Geriatr. 2009;49:176-179.

41. Sugawara N, Yasui-Furukori N, Umeda T, et al. Comparison of ankle-brachial pressure index and pulse wave velocity as markers of cognitive function in a community-dwelling population. BMC psychiatry. 2010;10:46.

42. Chrysohoou C, Psaltopoulou T, Panagiotakos D, et al. Aortic elastic properties and cognitive function in elderly individuals: The Ikaria Study. Maturitas. 2012.

43. Davis RN, Massman PJ, Doody RS. Effects of blood pressure on neuropsychological functioning in Alzheimer's disease. Arch Clin Neuropsychol. 2003;18:19-32.

44. Giang LM, Tighiouart H, Lou KV, et al. Measures of blood pressure and cognition in dialysis patients. Hemodial Int. 2013;17:24-31.

45. Kalaitzidis RG, Karasavvidou D, Tatsioni A, et al. Risk factors for cognitive dysfunction in CKD and hypertensive subjects. Int Urol Nephrol. 2013;45:1637-1646.

46. Molander L, Gustafson Y, Lovheim H. Low blood pressure is associated with cognitive impairment in very old people. Dement Geriatr Cogn Disord. 2010;29:335-341.

47. Obisesan TO, Obisesan OA, Martins S, et al. High blood pressure, hypertension, and high pulse pressure are associated with poorer cognitive function in persons aged 60 and older: the Third National Health and Nutrition Examination Survey. J Am Geriatr Soc. 2008;56:501-509.

48. Pase MP, Stough C, Grima NA, et al. Blood pressure and cognitive function: the role of central aortic and brachial pressures. Psychol Sci. 2013;24:2173-2181.

49. Raz N, Dahle CL, Rodrigue KM, Kennedy KM, Land S. Effects of age, genes, and pulse pressure on executive functions in healthy adults. Neurobiol Aging. 2011;32:1124-1137.

50. Robbins MA, Elias MF, Elias PK, Budge MM. Blood pressure and cognitive function in an AfricanAmerican and a Caucasian-American sample: the Maine-Syracuse Study. Psychosom Med. 2005;67:707714.

51. Sabayan B, Oleksik AM, Maier AB, et al. High blood pressure and resilience to physical and cognitive decline in the oldest old: the Leiden 85-plus Study. J Am Geriatr Soc. 2012;60:2014-2019.

52. Tsivgoulis G, Alexandrov AV, Wadley VG, et al. Association of higher diastolic blood pressure levels with cognitive impairment. Neurology. 2009;73:589-595.

53. van Bruchem-Visser RL, Mattace-Raso FU, van der Cammen TJ. High systolic and pulse pressure levels are associated with better cognitive performance in patients with probable Alzheimer's disease: a cross- 
sectional observational study in a geriatric outpatient population. Dement Geriatr Cogn Disord. 2009;28:320-324.

54. Yasar S, Ko JY, Nothelle S, Mielke MM, Carlson MC. Evaluation of the effect of systolic blood pressure and pulse pressure on cognitive function: the Women's Health and Aging Study II. PLoS One. 2011;6:e27976.

55. Benetos A, Watfa G, Hanon O, et al. Pulse wave velocity is associated with 1-year cognitive decline in the elderly older than 80 years: the PARTAGE study.J Am Med Dir Assoc. 2012;13:239-243.

56. Waldstein SR, Rice SC, Thayer JF, Najjar SS, Scuteri A, Zonderman AB. Pulse pressure and pulse wave velocity are related to cognitive decline in the Baltimore Longitudinal Study of Aging. Hypertension. 2008;51:99-104.

57. Zeki Al Hazzouri A, Newman AB, Simonsick E, et al. Pulse wave velocity and cognitive decline in elders: the Health, Aging, and Body Composition study. Stroke. 2013;44:388-393.

58. Freitag $\mathrm{MH}$, Peila R, Masaki K, et al. Midlife pulse pressure and incidence of dementia: the HonoluluAsia Aging Study. Stroke. 2006;37:33-37.

59. Lee AT, Chan WC, Chiu HF, et al. Widened pulse pressure is a potential risk factor for significant cognitive impairment among community-dwelling Chinese younger old people. J Alzheimers Dis. 2013;35:687696.

60. Morris MC, Scherr PA, Hebert LE, Glynn RJ, Bennett DA, Evans DA. Association of incident Alzheimer disease and blood pressure measured from 13 years before to 2 years after diagnosis in a large community study. Arch Neurol. 2001;58:1640-1646.

61. Ogunniyi A, Lane KA, Baiyewu O, et al. Hypertension and incident dementia in community-dwelling elderly Yoruba Nigerians. Acta Neurol Scand. 2011;124:396-402.

62. Peters R, Beckett N, Fagard R, et al. Increased pulse pressure linked to dementia: further results from the Hypertension in the Very Elderly Trial - HYVET. J Hypertens. 2013;31:1868-1875.

63. Qiu C, Winblad B, Viitanen M, Fratiglioni L. Pulse pressure and risk of Alzheimer disease in persons aged 75 years and older: a community-based, longitudinal study. Stroke. 2003;34:594-599.

64. Taylor C, Tillin T, Chaturvedi N, et al. Midlife hypertensive status and cognitive function 20 years later: the Southall and Brent revisited study. J Am Geriatr Soc. 2013;61:1489-1498.

65. Yang YH, Roe CM, Morris JC. Relationship between late-life hypertension, blood pressure, and Alzheimer's disease. Am J Alzheimers Dis Other Demen. 2011;26:457-462. 


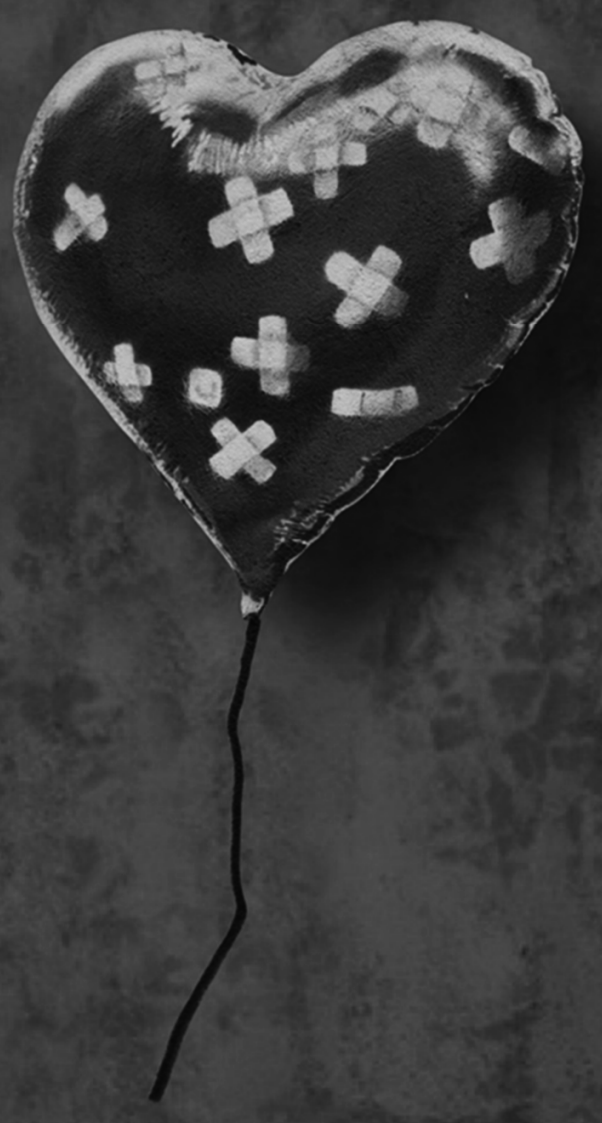

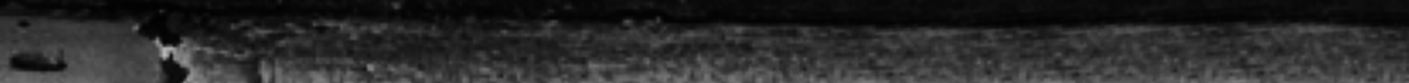
1. 1. 1. 


\section{Chapter 6}

\section{Cerebral small vessel disease and}

association with a higher incidence of depressive symptoms in a general elderly population:

\section{The AGES-Reykjavik Study}

TT van Sloten, S Sigurdsson, MA van Buchem, CL Phillips, PV Jonsson, J Ding, MT Schram, TB Harris, V Gudnason, LJ Launer 
138 | Chapter 6

\begin{abstract}

\section{Background}

The vascular depression hypothesis postulates that cerebral small vessel disease (CSVD) leads to depressive symptoms via disruption of brain structures involved in mood regulation. However, longitudinal data on the association between CSVD and depressive symptoms are scarce. We investigated the association between CSVD and incident depressive symptoms.
\end{abstract}

\title{
Methods
}

Longitudinal data are from the AGES-Reykjavik study of 1,949 participants free of dementia and without baseline depressive symptoms (74.6 years; $56.6 \%$ women). MRI markers of CSVD, detected at baseline (2002 to 2006) and follow-up (2007 to 2011), included white matter hyperintensity volume (WMHV), subcortical infarcts, cerebral microbleeds, Virchow-Robin spaces and total brain parenchyma volume. Incident depressive symptoms were defined by the 15-item Geriatric Depression Scale (GDS-15) score $\geq 6$ and/or use of antidepressant medication.

\section{Results}

Depressive symptoms occurred in $10.1 \%$ of the participants. The association for a greater onset of depressive symptoms was significant for participants having a 1 standard deviation (SD) increase in WMHV over time, new subcortical infarcts, new Virchow-Robin spaces, a 1 SD lower total brain volume at baseline, or a 1 SD decreased total brain volume over time, after adjustments for cognitive function, socio-demographic and cardiovascular factors. Results were qualitatively similar when change in the GDS-15 over time was used as the outcome instead of incident depressive symptoms.

\section{Conclusion}

Most markers of progression of CSVD over time and some markers of baseline CSVD are associated with concurrently developing new depressive symptoms. This study supports the vascular depression hypothesis. 


\section{Introduction}

Depressive symptoms are often present in older individuals, ${ }^{1-4}$ and are associated with an increase in morbidity and risk for mortality. 5,6 The pathobiology of late-life depressive symptoms is incompletely understood, but it has been suggested that cerebral small vessel disease (CSVD) is involved. ${ }^{7,8}$ The vascular depression hypothesis postulates that CSVD leads to depressive symptoms via disruption of deep and frontal brain structures or their connecting pathways involved in mood regulation. ${ }^{7,8}$

However, longitudinal data ${ }^{9-17}$ on the association between CSVD and depressive symptoms are limited and findings are mixed. Two previous studies ${ }^{12,13}$ found an association between markers of CSVD and incident depressive symptoms. In addition, three studies $9,10,15,16$ showed an association with increased severity or recurrence of depressive symptoms at follow-up, but not with incident depressive symptoms, whereas two other studies ${ }^{11,17}$ did not find any association with depressive symptoms. These mixed findings may be due to study differences in sample size, follow-up duration, method of depression assessment (clinical interview ${ }^{12-15}$ vs. questionnaire ${ }^{9-11,16,17}$ ), evaluation of different symptom clusters, and/or the source of populations investigated (selected ${ }^{9,10,12,17}$ vs. community-based samples $\left.{ }^{11,13-16}\right)$. In addition, some studies did not adjust the results for potential important confounders, such as cognitive function ${ }^{11,13,14,17}$ and cardiovascular factors. ${ }^{12,15,17}$

In view of the above, we investigated, in a large well characterized cohort, the prospective association between, on the one hand, markers of CSVD (white matter hyperintensity volume [WMHV], subcortical infarcts, cerebral microbleeds, Virchow-Robin spaces and lower total brain parenchyma volume) and, on the other, incident depressive symptoms. We additionally investigated whether any such association was stronger for CSVD in brain regions involved in mood regulation (deep and frontal) as compared to other regions (temporal and occipitoparietal). 


\section{Methods}

\section{Participants}

For the present study, we used longitudinal data from the Age, Gene/Environment Susceptibility (AGES)-Reykjavik Study. The AGES-Reykjavik Study is a population-based cohort study originating from the Reykjavik Study, as described fully elsewhere. ${ }^{18}$ Briefly, from 2002 to 2006, 5,764 surviving participants of the Reykjavik Study were examined. From 2007 to 2011 there was a follow-up examination of all surviving participants who agreed to participate $(n=3,316)$. Reasons for not attending the follow-up examination included: death ( $n=1,039)$; refusal $(n=1,198)$; and lost to follow-up (could not be contacted by any means; $n=211$ ). The AGES-Reykjavik Study was approved by the National Bioethics Committee in Iceland (approval number: VSN-00-063), and by the National Institute on Aging Intramural Institutional Review Board. After complete description of the study to the subject, written informed consent was obtained.

\section{Depressive symptoms}

Depressive symptoms were assessed on all participants, with the 15-item Geriatric Depression Scale (GDS-15; score range, 0-15). ${ }^{19,20}$ Incident depressive symptoms were defined as a predefined GDS-15 cut-off score of 6 or higher ${ }^{19,20}$ at follow-up and/or new use of antidepressant medication (tricyclics, selective serotonin reuptake inhibitors, other nontricyclics and monoamino oxidate inhibitors) assessed from medication bottles brought to the clinic. Individuals were excluded for the present analysis if they had depressive symptoms at baseline (GDS-15 score of 6 or higher and/or use of antidepressant medication at baseline).

\section{Brain MRI measures}

Image acquisition. All eligible participants were offered a high-resolution brain MRI acquired on a study-dedicated 1.5-T system (Signa Twinspeed, General Electric Medical Systems). The same imaging protocol was used in the 2002-2006 and 2007-2011 examinations, described elsewhere, ${ }^{21,22}$ and included the following sequences: 3D spoiledgradient recalled T1-weighted, proton density/T2-weighted fast spin-echo, fluidattenuated inversion recovery (FLAIR) and T2*-weighted gradient-echo type echoplanar (GRE-EPI). All images were acquired to give full brain coverage with slices angled parallel to the anterior commissure-posterior commissure line in order to give reproducible image views in the oblique-axial plane.

Image analysis. Several markers of CSVD were evaluated. WMHV and total brain parenchyma volume (an indicator of cerebral atrophy) were computed automatically with a previously described image analysis pipeline ${ }^{23}$ and were expressed as the percentage of total intracranial volume. Lower total brain parenchyma volume was considered to be a 
marker of CSVD, as CSVD leads to generalized loss of brain parenchyma via, amongst others, microinfarcts ${ }^{24}$ and loss of white matter integrity. ${ }^{25}$ Subcortical infarcts were evaluated as described previously ${ }^{21}$ and defined as brain parenchyma defects not extending into the cortex, with a minimum diameter of $4 \mathrm{~mm}$ and a signal intensity equal to cerebrospinal fluid on all pulse sequences (T2-weighted, proton density-weighted and FLAIR), and surrounded by an area of high signal intensity on FLAIR images. Parenchymal defects in the subcortical area with evidence of hemosiderin on the T2*-weighted GRE-EPI scan were labelled as resorbed hematomas and were excluded from the definition of subcortical infarcts. Virchow-Robin spaces were evaluated separately and defined as defects in the subcortical area without a rim or area of high signal intensity on FLAIR and without evidence of hemosiderin on the T2*-weighted GRE-EPI scan. Presence of VirchowRobin spaces was considered to be a marker of CSVD, as they are associated with endothelial dysfunction, which may play a role in the pathogenesis of CSVD. ${ }^{26}$ Cerebral microbleeds were defined as focal areas of signal void within the brain parenchyma visible on T2*-weighted GRE-EPI scans and were identified as described previously. ${ }^{22}$

\section{Potential confounding variables}

As described elsewhere, ${ }^{18}$ dementia case-finding was conducted at baseline and at followup according to a three step procedure. Diagnosis of dementia (all sub-types) was made according to the Diagnostic and Statistical Manual of Mental Disorders ( $4^{\text {th }}$ edition) by a panel that included a geriatrician, a neurologist, a neuropsychologist and a neuroradiologist. The following variables were assessed by questionnaire: education (categorized into primary, secondary and college/university education), smoking history (ever vs. never), alcohol intake (high [>median] vs. low consumers [<median]; median for women 3.2 and for men $8.0 \mathrm{~g} /$ week, respectively) and anxiety symptoms (presence vs. absence). Presence of anxiety symptoms was defined by a positive response to any of the following questions: "In the past month, have you felt anxious or frightened?"; "Were there times lately that you felt anxious?"; "Are there special situations that make you anxious?"; and "Have you ever had attacks of fear or panic?". Presence of anxiety symptoms is a potential confounder, because anxiety symptoms are frequently present in individuals with depression, and are associated with cerebrovascular disease independently of depression. ${ }^{27,28}$ Gait speed, a measure of physical performance, ${ }^{29}$ was the time needed to walk 6 meters at a usual pace. Hypertension was defined as systolic pressure $>140 \mathrm{mmHg}$, diastolic pressure $>90 \mathrm{mmHg}$ and/or use of anti-hypertensive medication. Body mass index (BMI) was calculated as measured weight divided by height squared. Diabetes was defined as a self-reported doctor's diagnosis of diabetes, use of blood glucose-lowering medication and/or fasting blood glucose level $\geq 7.0 \mathrm{mmol} / \mathrm{l}$. Coronary calcium score (categorized into sex-specific quartiles), a measure of atherosclerosis, was based on Computed Tomography. ${ }^{30}$ The Digit Symbol Substitution 
Test (DSST), a measure of psychomotor speech, and the Mini Mental State Examination (MMSE), a measure of global cognitive function, were also administered..$^{18,30}$

\section{Analytic sample}

Of the 3,316 participants who attended the follow-up examination, 709 had missing MRI data and another 195 had missing data on depressive symptoms at baseline or at followup. Missing MRI data was due to contraindications $(n=278)$, refusal/nonattendance ( $n=360$ ), or technical reasons (no qualitatively acceptable data available for all necessary sequences; $n=71$ ). In the remaining 2,412 participants, 138 were excluded because of a diagnosis of dementia at baseline $(n=31)$ or follow-up $(n=107)$. Finally, participants were excluded with presence of depressive symptoms at baseline $(n=325)$. The final study sample thus consisted of 1,949 participants. Participants excluded for the present analysis were more likely to be older (75.6 vs. 74.6 years), female (60.8 vs. $56.6 \%$ ), less educated (for primary school or less: 23.3 vs. 18.9\%) and to have diabetes (12.4 vs. 9.1\%), hypertension ( 82.0 vs. $76.9 \%$ ) and/or stroke (9.1 vs. $5.2 \%$ ) (P-value for all <.05).

\section{Statistical analysis}

The percentage of missing values on potential confounders was minimal (maximum, 1.7\%). We imputed those data using multiple imputation chained equations (10 datasets). ${ }^{31}$ WMHV was logarithmically transformed to normalize its skewed distribution.

The statistical analysis proceeded in several stages. Logistic regression analysis was used to estimate the association between markers of baseline CSVD (baseline WMHV, expressed per 1 higher standard deviation [per $+1 \mathrm{SD}$ ], presence of any (coded 0,1 ) subcortical infarcts, cerebral microbleeds and Virchow-Robin spaces and total brain parenchyma volume, expressed per 1 lower SD [per -1 SD]) and incident depressive symptoms. Analyses were repeated looking prospectively at change in, or progression of, markers of CSVD as the determinant and development of depressive symptoms over the same period. These markers were an increase in WMHV from baseline values (per $+1 \mathrm{SD}$ ), any incident (coded 0,1 ) subcortical infarcts, cerebral microbleeds and Virchow-Robin spaces, and a decrease in total brain parenchyma volume from baseline values (per -1 SD).

We repeated the above analyses for a priori selected brain regions: deep (sub-cortical) areas (internal and external capsules, thalamus, striatum, hippocampus and amygdala combined), and frontal, temporal and occipitoparietal lobes. To efficiently summarize the pathology in each of these regions, we created a dichotomous composite score. This was calculated by assigning one point per CSVD marker based on the following cut-offs: regional WMHV, quartile 4 vs. quartiles 1 to 3; subcortical infarcts, cerebral microbleeds and Virchow-Robin spaces, $\geq 1$ vs. 0 lesion(s) per region; and regional brain parenchyma volume, quartile 1 vs. quartiles 2 to 4 . The points for each marker were combined to 
compute a dichotomous composite score per region, which indicated high ( $\geq 2$ points) or low (0 or 1 point(s)) burden of CSVD. A separate composite score was computed for baseline and progression over time of CSVD, respectively.

In addition, linear regression was used to evaluate the association between markers of CSVD and change of the GDS-15 score over time as the outcome

All models were adjusted for the following potential confounders: baseline age, sex, DSST score, MMSE score, education level, presence of anxiety symptoms, gait speed, alcohol use, smoking, diabetes, BMI, hypertension, coronary calcium score, head coil and followup time (model 1); and additionally for baseline GDS-15 score (model 2). The composite scores for each region were additionally mutually adjusted for each other, with the exception of the scores for the frontal and deep brain region, which were not adjusted for each other, because both regions are thought to be involved in mood regulation. ${ }^{7,8}$ We did not adjust the results for total disease load, because total disease load includes the load per investigated region (as indicated by the composite scores) and, thus, can be considered an overadjustment. ${ }^{32}$

To test the robustness of the results, several sensitivity analyses were done. Logistic regression analyses were repeated with incident depressive symptoms defined only as GDS-15 score of 6 or higher as the outcome, irrespective of new use of antidepressant medication. To minimize the potential confounding effect of stroke, analyses were repeated after excluding individuals with baseline stroke or incident stroke during followup. In addition, to assess the possibility that depressive symptoms lead to CSVD (reverse 'causality'), analyses were repeated with baseline presence of depressive symptoms as the exposure variable (we did not exclude individuals with baseline depressive symptoms; $\mathrm{n}=325$ ) and markers of progression of CSVD over time as the outcome. All analyses were conducted with PASW Statistics (version 21, IBM, Chicago, Illinois, USA).

\section{Results}

The mean age of the study population at baseline was 74.6 years and $56.6 \%$ were women (Table 6.1). In total, $10.1 \%(n=197)$ of the participants had incident depressive symptoms, of whom $38.1 \%(n=75)$ had a GDS-15 score of 6 or higher and $70.6 \%(n=139)$ had started using antidepressant medication (22 used tricyclics, 85 selective serotonin reuptake inhibitors and 43 other antidepressant medication). The mean time between the baseline and follow-up examination was $5.2 \pm 0.2$ years. 
Table 6.1. Characteristics of both the total study population $(n=1,949)$ and according to incident depressive symptoms

\begin{tabular}{|c|c|c|c|}
\hline & $\begin{array}{l}\text { Total study } \\
\text { population } \\
(n=1,949)\end{array}$ & $\begin{array}{l}\text { Individuals without } \\
\text { incident depressive } \\
\text { symptoms }(n=1,752)\end{array}$ & $\begin{array}{c}\text { Individuals with } \\
\text { incident depressive } \\
\text { symptoms }(n=197)\end{array}$ \\
\hline \multicolumn{4}{|l|}{ General baseline characteristics } \\
\hline Age (years) & $74.6 \pm 4.6$ & $74.5 \pm 4.6$ & $75.2 \pm 4.9$ \\
\hline Women & $56.6(1,103)$ & $55.4(971)$ & $67.0(132)$ \\
\hline \multicolumn{4}{|l|}{ Education level } \\
\hline Primary & $18.9(367)$ & $18.5(324)$ & $21.9(43)$ \\
\hline Secondary & $51.3(996)$ & $51.3(897)$ & $50.5(99)$ \\
\hline College / University & $29.9(580)$ & $30.1(526)$ & $27.6(54)$ \\
\hline Presence of anxiety symptoms & $26.7(521)$ & $24.8(435)$ & $43.7(86)$ \\
\hline \multicolumn{4}{|l|}{ Gait speed $(\mathrm{m} / \mathrm{s})$} \\
\hline Baseline & $0.98(0.92-1.13)$ & $0.98(0.91-1.13)$ & $0.92(0.84-1.08)$ \\
\hline Change over time & $-0.09 \pm 0.23$ & $-0.08 \pm 0.22$ & $-0.13 \pm 0.25$ \\
\hline \multicolumn{4}{|l|}{ Digit Symbol Substitution Test score } \\
\hline Baseline score & $33 \pm 10$ & $33 \pm 10$ & $31 \pm 11$ \\
\hline Change over time & $-3 \pm 5$ & $-3 \pm 5$ & $-4 \pm 6$ \\
\hline \multicolumn{4}{|l|}{ Mini Mental State Examination score } \\
\hline Baseline score & $28(27-29)$ & $28(27-29)$ & $28(26-29)$ \\
\hline Change over time & $-1(-3-1)$ & $-1(-3-1)$ & $-2(-4-1)$ \\
\hline \multicolumn{4}{|l|}{ Smoking status } \\
\hline Ever & $55.9(1,090)$ & $55.2(967)$ & $62.4(123)$ \\
\hline Never & $44.1(859)$ & $44.8(785)$ & $37.6(74)$ \\
\hline Alcohol use (g/week) & $3(0-16)$ & $3(0-16)$ & $3(0-16)$ \\
\hline Body mass index $\left(\mathrm{kg} / \mathrm{m}^{2}\right)$ & $27.2 \pm 4.0$ & $27.1 \pm 4.0$ & $27.5 \pm 4.3$ \\
\hline Diabetes & $9.1(177)$ & $9.1(160)$ & $8.6(17)$ \\
\hline Hypertension & $76.9(1,499)$ & $76.9(1,347)$ & $77.2(152)$ \\
\hline Coronary calcium score (Agatston score) & $212(28-744)$ & $211(30-751)$ & $193(26-693)$ \\
\hline Stroke & $5.0(98)$ & $4.9(85)$ & $6.6(13)$ \\
\hline \multicolumn{4}{|l|}{ Brain MRI markers } \\
\hline \multicolumn{4}{|l|}{ Total white matter hyperintensity } \\
\hline Baseline volume & $6 \pm 8$ & $5 \pm 8$ & $7 \pm 8$ \\
\hline \multicolumn{4}{|l|}{ Change over time } \\
\hline \multicolumn{4}{|l|}{ Subcortical infarcts } \\
\hline Baseline presence of any infarct(s) & 7.1 (139) & $6.7(117)$ & $11.2(22)$ \\
\hline Any new infarct(s) over time & $4.0(77)$ & $3.6(63)$ & $7.1(14)$ \\
\hline \multicolumn{4}{|l|}{ Cerebral microbleeds } \\
\hline Baseline presence of any microbleed(s) & $17.3(337)$ & $17.2(301)$ & $18.3(36)$ \\
\hline Any new microbleed(s) over time & $17.9(348)$ & $17.6(308)$ & $20.3(40)$ \\
\hline \multicolumn{4}{|l|}{ Virchow-Robin spaces } \\
\hline Baseline presence of any space(s) & $15.9(309)$ & $15.8(277)$ & $16.2(32)$ \\
\hline Any new space(s) over time & $2.6(51)$ & $2.2(39)$ & $6.1(12)$ \\
\hline \multicolumn{4}{|l|}{ Total brain parenchyma volume (ml) } \\
\hline Baseline volume & $1099 \pm 104$ & $1100 \pm 104$ & $1087 \pm 106$ \\
\hline Change over time & $-31 \pm 23$ & $-31 \pm 24$ & $-35 \pm 20$ \\
\hline
\end{tabular}

Data are presented as percentage of participants $(n)$, mean \pm standard deviation or median (interquartile range). 
The results of the analysis with markers of baseline CSVD showed that subcortical infarcts and a lower total brain parenchyma volume were statistically significantly associated with a higher incidence of depressive symptoms, after adjustment for potential confounders (Table 6.2, model 1). Further adjustment for baseline GDS-15 scores did not materially change these results (model 2).

In addition, the results of the analysis with markers of progression of CSVD over time showed that an increase in WMHV over time, incident subcortical infarcts and VirchowRobin spaces and a decrease in total brain parenchyma volume over time were statistically significantly associated with a higher incidence of depressive symptoms (Table 6.2, models 1 and 2).

Table 6.2. Associations between markers of baseline and progression over time of cerebral small vessel disease and incident depressive symptoms

\begin{tabular}{|c|c|c|c|}
\hline \multirow[t]{2}{*}{ Determinants } & \multirow[t]{2}{*}{ Model } & \multicolumn{2}{|c|}{ Incident depressive symptoms } \\
\hline & & Odds ratio $(95 \% \mathrm{Cl})$ & P-value \\
\hline \multicolumn{4}{|l|}{ White matter hyperintensity volume (\% ICV) } \\
\hline \multirow[t]{2}{*}{ Per +1 SD volume at baseline } & 1 & $1.04(0.89 ; 1.21)$ & .67 \\
\hline & 2 & $1.02(0.88 ; 1.19)$ & .78 \\
\hline \multirow[t]{2}{*}{ Per +1 SD volume change over time } & 1 & $1.24(1.09 ; 1.42)$ & .002 \\
\hline & 2 & $1.21(1.06 ; 1.39)$ & .007 \\
\hline \multicolumn{4}{|l|}{ Subcortical infarcts } \\
\hline \multirow[t]{2}{*}{ Baseline presence of any infarct(s) vs. no infarcts } & 1 & $1.90(1.15 ; 3.14)$ & .012 \\
\hline & 2 & $1.83(1.10 ; 3.05)$ & .021 \\
\hline \multirow[t]{2}{*}{ Any new infarct(s) over time vs. no new infarcts } & 1 & $2.39(1.28 ; 4.48)$ & .007 \\
\hline & 2 & $2.31(1.21 ; 4.39)$ & .011 \\
\hline \multicolumn{4}{|l|}{ Cerebral microbleeds } \\
\hline \multirow[t]{2}{*}{ Baseline presence of any microbleed(s) vs. no microbleeds } & 1 & $1.15(0.77 ; 1.72)$ & .50 \\
\hline & 2 & $1.10(0.73 ; 1.66)$ & .64 \\
\hline \multirow[t]{2}{*}{ Any new microbleed(s) over time vs. no new microbleeds } & 1 & $1.31(0.92 ; 1.87)$ & .16 \\
\hline & 2 & $1.36(0.98 ; 1.86)$ & .12 \\
\hline \multicolumn{4}{|l|}{ Virchow-Robin spaces } \\
\hline \multirow[t]{2}{*}{ Baseline presence of any space(s) vs. no spaces } & 1 & $1.09(0.73 ; 1.61)$ & .69 \\
\hline & 2 & $1.08(0.70 ; 1.66)$ & .71 \\
\hline \multirow[t]{2}{*}{ Any new space(s) over time vs. no new spaces } & 1 & $3.44(1.71 ; 6.91)$ & .001 \\
\hline & 2 & $3.75(1.83 ; 7.68)$ & $<.001$ \\
\hline \multicolumn{4}{|l|}{ Total brain parenchyma volume (\% ICV) } \\
\hline \multirow[t]{2}{*}{ Per -1 SD volume at baseline } & 1 & $1.23(1.05 ; 1.45)$ & .012 \\
\hline & 2 & $1.23(1.04 ; 1.45)$ & .017 \\
\hline \multirow[t]{2}{*}{ Per -1 SD volume change over time } & 1 & $1.30(1.08 ; 1.57)$ & .006 \\
\hline & 2 & $1.32(1.09 ; 1.59)$ & .004 \\
\hline
\end{tabular}

Model 1: adjusted for baseline age, sex, Digit Symbol Substitution Test score, Mini Mental State Examination score, education level, presence of anxiety symptoms, gait speed, alcohol use, smoking, diabetes, body mass index, hypertension, coronary calcium score, head coil and follow-up time. Model 2: additionally adjusted for baseline 15-item geriatric depression scale (GDS-15) score.

$\mathrm{Cl}=$ confidence interval; $\mathrm{SD}=$ standard deviation; $\mathrm{ICV}=$ intracranial volume. 
The composite scores of baseline (Figure 6.1, Panel A) and the change in pathology over time (Panel B) in the deep brain region was most strongly and statistically significantly associated with a higher incidence of depressive symptoms. In addition, the composite baseline score of the frontal brain region was statistically significantly associated with a higher incidence of depressive symptoms.

A

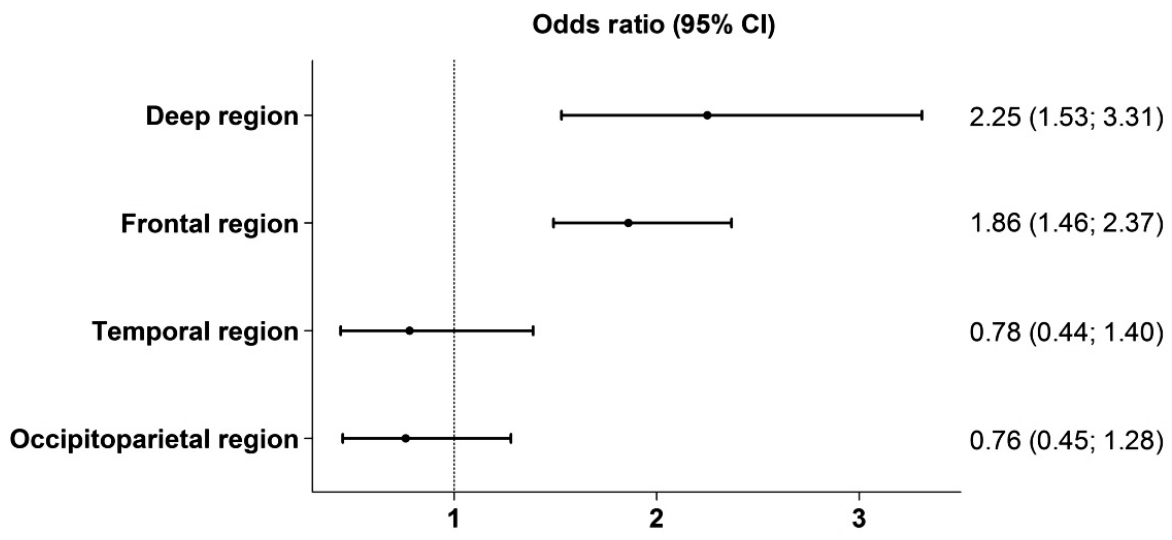

B

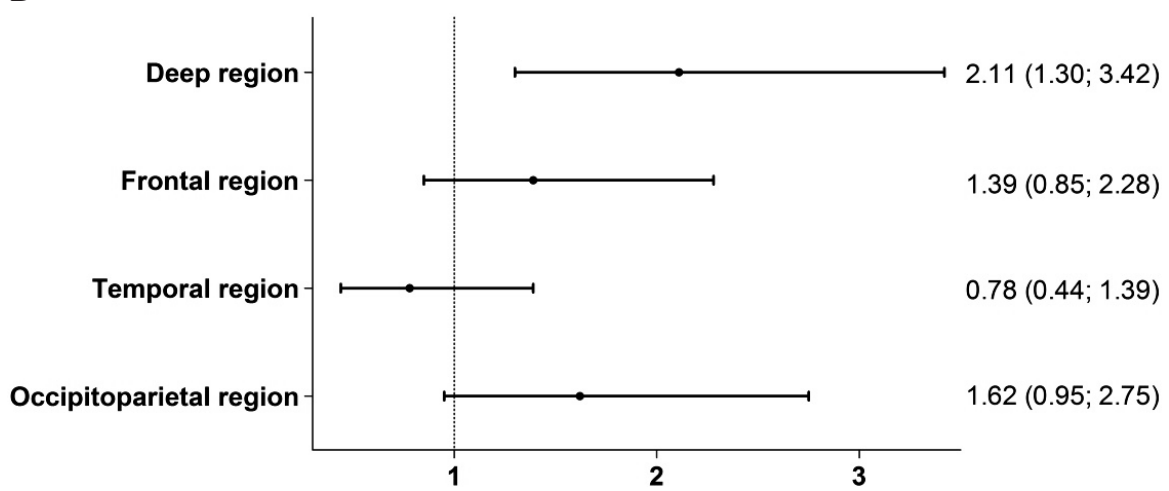

Figure 6.1. Associations between brain region-specific composite scores of baseline (panel A) and progression over time (panel B) of cerebral small vessel disease and incident depressive symptoms. Composite scores indicate high versus low burden of cerebral small vessel disease per region. The composite scores were mutually adjusted for each other (except for the frontal and deep brain region which were not associated for each other) and for all potential confounders. For further explanation: see text.

The results of the analyses with change of the GDS-15 score over time as the outcome were qualitatively similar to the results of the analyses with incident depressive symptoms (Table 6.3, models 1 and 2). The association for a greater GDS-15 score over time was statistically significant for higher baseline WMHV, an increase in WMHV over time, baseline and incident subcortical infarcts, incident cerebral microbleeds, baseline and incident Virchow-Robin spaces and a decrease in total brain parenchyma volume over time (Table 6.3, models 1 and 2). 
Table 6.3. Associations between markers of baseline and progression over time of cerebral small vessel disease and change of the GDS-15 score over time

\begin{tabular}{|c|c|c|c|}
\hline \multirow[t]{2}{*}{ Determinants } & \multirow[t]{2}{*}{ Model } & \multicolumn{2}{|c|}{ Incident depressive symptoms } \\
\hline & & $\beta$ coefficient $(95 \% \mathrm{Cl})$ & P-value \\
\hline \multicolumn{4}{|l|}{ White matter hyperintensity volume (\% ICV) } \\
\hline \multirow[t]{2}{*}{ Per +1 SD volume at baseline } & 1 & $0.11(0.04 ; 0.18)$ & .003 \\
\hline & 2 & $0.11(0.04 ; 0.18)$ & .001 \\
\hline \multirow[t]{2}{*}{ Per +1 SD volume change over time } & 1 & $0.16(0.09 ; 0.24)$ & $<.001$ \\
\hline & 2 & $0.20(0.13 ; 0.26)$ & $<.001$ \\
\hline \multicolumn{4}{|l|}{ Subcortical infarcts } \\
\hline \multirow[t]{2}{*}{ Baseline presence of any infarct(s) vs. no infarcts } & 1 & $0.27(-0.01 ; 0.54)$ & .05 \\
\hline & 2 & $0.34(0.08 ; 0.59)$ & .01 \\
\hline \multirow[t]{2}{*}{ Any new infarct(s) over time vs. no new infarcts } & 1 & $0.34(-0.02 ; 0.70)$ & .07 \\
\hline & 2 & $0.40(0.06 ; 0.74)$ & .02 \\
\hline \multicolumn{4}{|l|}{ Cerebral microbleeds } \\
\hline \multirow[t]{2}{*}{ Baseline presence of any microbleed(s) vs. no microbleeds } & 1 & $-0.03(-0.22 ; 0.15)$ & .73 \\
\hline & 2 & $-0.02(-0.18 ; 0.17)$ & .98 \\
\hline \multirow[t]{2}{*}{ Any new microbleed(s) over time vs. no new microbleeds } & 1 & $0.20(0.02 ; 0.38)$ & .03 \\
\hline & 2 & $0.18(0.01 ; 0.36)$ & .04 \\
\hline \multicolumn{4}{|l|}{ Virchow-Robin spaces } \\
\hline \multirow[t]{2}{*}{ Baseline presence of any space(s) vs. no spaces } & 1 & $0.19(-0.00 ; 0.38)$ & .05 \\
\hline & 2 & $0.18(0.00 ; 0.36)$ & $<.05$ \\
\hline \multirow[t]{2}{*}{ Any new space(s) over time vs. no new spaces } & 1 & $0.80(0.37 ; 1.22)$ & $<.001$ \\
\hline & 2 & $0.74(0.34 ; 1.13)$ & .001 \\
\hline \multicolumn{4}{|l|}{ Total brain parenchyma volume (\% ICV) } \\
\hline \multirow[t]{2}{*}{ Per -1 SD volume at baseline } & 1 & $0.04(-0.04 ; 0.12)$ & .32 \\
\hline & 2 & $0.06(-0.01 ; 0.14)$ & .11 \\
\hline \multirow[t]{2}{*}{ Per -1 SD volume change over time } & 1 & $0.08(0.02 ; 0.15)$ & .02 \\
\hline & 2 & $0.08(0.01 ; 0.15)$ & .02 \\
\hline
\end{tabular}

Model 1: adjusted for baseline age, sex, Digit Symbol Substitution Test score, Mini Mental State Examination score, education level, presence of anxiety symptoms, gait speed, alcohol use, smoking, diabetes, body mass index, hypertension, coronary calcium score, head coil and follow-up time. Model 2: additionally adjusted for baseline 15-item geriatric depression scale (GDS-15) score. Abbreviations as in Table 6.2.

\section{Sensitivity analyses}

Results were qualitatively similar when we repeated the analyses with incident depressive symptoms defined only as a GDS-15 score of 6 or higher, irrespective of new use of antidepressant medication (see supplement material, Table S6.1). When we excluded individuals with baseline stroke or incident stroke during follow-up ( $n=152)$, results did not materially change (see supplemental material, Table S6.2). Presence of depressive symptoms at baseline was not statistically significantly associated with markers of progression of CSVD (see supplemental material, Table S6.3). 


\section{Discussion}

The present study investigated the association between markers of CSVD and incident depressive symptoms and had two main findings. First, various markers of CSVD were associated with a higher incidence of depressive symptoms. This association was statistically significant for an increase in WMHV over time, baseline and incident subcortical infarcts, incident Virchow-Robin spaces, a lower total brain parenchyma volume at baseline and a decrease in total brain parenchyma volume over time. These results were independent of cognitive function, education level, physical performance, anxiety symptoms and cardiovascular factors. In addition, results were qualitatively similar when change in the GDS-15 score over time was used as the outcome instead of incident depressive symptoms. Second, CSVD located in the deep brain region was, as compared to other brain regions, more strongly associated with a higher incidence of depressive symptoms. To our knowledge, this is the clearest demonstration to date that CSVD is a risk factor for depressive symptoms.

Our findings are in accordance with previous cross-sectional studies ${ }^{13,14,33}$ which consistently have shown an association between markers of CSVD and depression. In addition, two previous, smaller longitudinal studies, the 3City-Dijon study ${ }^{13}$ and the LADIS, ${ }^{12}$ found an association between higher WMHV at baseline and a higher incidence of depressive symptoms after a follow-up of 4 and 3 years, respectively. Furthermore, the SMART-Medea study ${ }^{9}$ found an association between lacunar infarcts in deep white matter tracts and an increased severity and more fluctuating course of depressive symptoms, in particular motivational/apathy related-symptoms of depression, during a follow-up of 3.5 years. In addition, the Cardiovascular Health Study ${ }^{16}$ and a neuroimaging substudy of the Rotterdam Study ${ }^{15}$ showed an association between higher WHMV and/or cerebral infarcts on the one hand and worsening and recurrence of depressive symptoms on the other after 4 years of follow-up. In contrast, two other longitudinal studies, a post-hoc analysis of a clinical trial ${ }^{17}$ and the Baltimore Longitudinal Study of Aging, ${ }^{11}$ did not find any association between WMHV at baseline and incident depressive symptoms. The latter studies were, however, relatively small $(n<550)^{11,17}$ and/or had a relatively short follow-up duration (<3 years), ${ }^{17}$ which may have led to an underestimation of the association between CSVD and depressive symptoms. The present study extends previous research because of its large population-based sample of older individuals, long follow-up duration, the comprehensive brain MRI assessment of a wide spectrum of CSVD markers determined at baseline and at follow-up, and the extensive characterization of participants which enabled us to adjust for a series of potential confounders.

CSVD may lead to depressive symptoms via damage to deep and frontal brain structures involved in mood regulation. ${ }^{7,8}$ In accordance, the present study found that CSVD in the 
deep brain region was, as compared to other regions, more strongly associated with a higher incidence of depressive symptoms, although the $95 \% \mathrm{Cl}$ of the OR for disease in the deep brain region did overlap with those of other regions.

Other underlying mechanisms may, however, explain the observed associations. First, it has been suggested that the association between CSVD and depressive symptoms exists because late-life depressive symptoms represent an early manifestation of (vascular) dementia. ${ }^{34}$ For the present study, however, we excluded individuals with dementia at baseline or at follow-up. In addition, results were adjusted for scores on the DSST and the MMSE, tests that evaluate multiple cognitive functions. ${ }^{35}$ Second, other factors may be independently related to both CSVD and depressive symptoms, such as anxiety, cardiovascular factors and stroke. The associations between CSVD and incident depressive symptoms were, however, independent of anxiety symptoms and cardiovascular factors. In addition, the associations did not materially change when we excluded individuals with stroke. Third, we cannot exclude the possibility that the observed associations reflect reverse causation. Indeed, previous studies ${ }^{36,37}$ have shown an association between depression and incident cardiovascular disease, including cerebrovascular disease. Although it is not fully understood how depression might lead to vascular disease, possible mechanisms include low-grade inflammation, endothelial dysfunction, platelet dysfunction and unfavorable lifestyle habits. ${ }^{38}$ It is unclear, however, why depression would lead to vascular disease in specific brain regions, e.g. the deep brain region involved in mood regulation. In addition, presence of depressive symptoms at baseline was not statistically significantly associated with markers of progression of CSVD over time in the present study, although the $95 \% \mathrm{Cls}$ of the effect estimates do not exclude the possibility of such an association. Fourth, it has been suggested that associations between CSVD and depression may be (partially) attributable to apathy. ${ }^{39}$ Apathy overlaps with depression, but may be a distinct syndrome. ${ }^{40}$ In the present study, we did not evaluate apathy and this issue needs further study.

The present study showed that most markers of progression of CSVD over time were associated with incident depressive symptoms, but only some markers of baseline CSVD. This may be due to the design of the present study with exclusion of individuals with depressive symptoms at baseline. This may have led to an underestimation of the association between baseline CSVD and development of depressive symptoms, but not between progression of CSVD over time and depressive symptoms, because individuals with depressive symptoms at baseline were most likely those with the strongest association between lifetime accumulation of CSVD (which is reflected by baseline CSVD) and depressive symptoms. 
We analyzed depressive symptoms both as a dichotomous and a continuous outcome. The results of these analyses were qualitatively similar, except that more associations were statistically significant with change in the continuous GDS-15 score than with the dichotomous incident depressive symptoms variable. This difference may be due to the fact that, in general, analyses with a continuous outcome have higher statistical power than analyses with a dichotomous outcome. Indeed, studying depression on a continuum has the merit that not only information on extremes is used, but that all available information is exploited.

There are a number of limitations to the present study. First, incident depressive symptoms were assessed by questionnaire and use of antidepressant medication, but not by a structured interview. Therefore, no information was available on clinical depression. Nevertheless, the sensitivity and specificity of questionnaire measures as compared to a depression diagnosis based on a structured interview are high $(>80 \%) .{ }^{20}$ Yet, the prevalence of depressive symptoms is greater, in particular in older individuals. ${ }^{2,4}$ Furthermore, late-life depressive symptoms, even in the absence of a diagnosis of a major depressive disorder, are associated with a greatly increased morbidity and mortality risk. 5,6 Second, misclassification of incident depressive symptoms may have occurred because antidepressant medication is also prescribed for other reasons. The results were, however, qualitatively similar when GDS-15 scores alone were used as the outcome. Third, the present study is the first to evaluate the association between brain region-specific composite scores and depressive symptoms, and further study is, therefore, needed to confirm the present findings (e.g. using voxel-based morphometric analysis). Fourth, a limitation of the analysis with markers of progression of CSVD over time as the determinant is that progression of CSVD and incident depressive symptoms occur in the same time interval and cannot be assigned to a given time point within this interval. Finally, we used cerebral atrophy and Virchow-Robin spaces as markers of CSVD. Cerebral atrophy is, however, an indirect measure of vascular disease and is also strongly determined by other factors, in particular the process of neurodegeneration. We therefore cannot exclude the possibility that the association between lower total brain parenchyma volume and depressive symptoms is due to factors other than CSVD. In addition, the etiology of Virchow-Robin spaces is currently incompletely understood, and this issue requires further study.

In conclusion, the present study shows that most markers of progression of CSVD over time and only some markers of baseline CSVD are independently associated with a concurrent development of higher incident depressive symptoms. From a clinical point of view, this association is important as it suggests that CSVD is a target for treatment and prevention strategies of late-life depression. Further study is needed to elucidate which 
Cerebral small vessel disease and incident depressive symptoms | 151

factors contribute to CSVD and whether such factors can be therapeutic targets for latelife depression. 


\section{References}

1. Byers AL, Yaffe K, Covinsky KE, Friedman MB, Bruce ML. High occurrence of mood and anxiety disorders among older adults: The National Comorbidity Survey Replication. Arch Gen Psychiatry. 2010;67:489-496.

2. Sutin AR, Terracciano A, Milaneschi $Y, A n Y$, Ferrucci L, Zonderman AB. The trajectory of depressive symptoms across the adult life span. JAMA Psychiatry. 2013;70:803-811.

3. Byers AL, Vittinghoff E, Lui LY, et al. Twenty-year depressive trajectories among older women. Arch Gen Psychiatry. 2012;69:1073-1079.

4. Luijendijk HJ, van den Berg JF, Dekker MJ, et al. Incidence and recurrence of late-life depression. Arch Gen Psychiatry. 2008;65:1394-1401.

5. Barefoot JC, Schroll M. Symptoms of depression, acute myocardial infarction, and total mortality in a community sample. Circulation. 1996;93:1976-1980.

6. Penninx BW, Geerlings SW, Deeg DJ, van Eijk JT, van Tilburg W, Beekman AT. Minor and major depression and the risk of death in older persons. Arch Gen Psychiatry. 1999;56:889-895.

7. Alexopoulos GS, Meyers BS, Young RC, Campbell S, Silbersweig D, Charlson M. 'Vascular depression' hypothesis. Arch Gen Psychiatry. 1997;54:915-922.

8. Krishnan KR, Hays JC, Blazer DG. MRI-defined vascular depression. Am J Psychiatry. 1997;154:497-501.

9. Grool AM, Gerritsen L, Zuithoff NP, Mali WP, van der Graaf Y, Geerlings MI. Lacunar infarcts in deep white matter are associated with higher and more fluctuating depressive symptoms during three years follow-up. Biol Psychiatry. 2013;73:169-176.

10. Grool AM, van der Graaf Y, Mali WP, Witkamp TD, Vincken KL, Geerlings MI. Location and progression of cerebral small-vessel disease and atrophy, and depressive symptom profiles: The Second Manifestations of ARTerial disease (SMART)-Medea study. Psychol Med. 2011:1-12.

11. Dotson VM, Zonderman AB, Kraut MA, Resnick SM. Temporal relationships between depressive symptoms and white matter hyperintensities in older men and women. Int J Geriatr Psychiatry. 2013;28:66-74.

12. Firbank MJ, Teodorczuk A, van der Flier WM, et al. Relationship between progression of brain white matter changes and late-life depression: 3-year results from the LADIS study. $\mathrm{Br} J$ Psychiatry. 2012;201:40-45.

13. Godin O, Dufouil C, Maillard P, et al. White matter lesions as a predictor of depression in the elderly: the 3C-Dijon study. Biol Psychiatry. 2008;63:663-669.

14. Ikram MA, Luijendijk HJ, Vernooij MW, et al. Vascular brain disease and depression in the elderly. Epidemiology. 2010;21:78-81.

15. Saavedra Perez HC, Direk N, Hofman A, Vernooij MW, Tiemeier H, Ikram MA. Silent brain infarcts: a cause of depression in the elderly? Psychiatry Res. 2013;211:180-182.

16. Steffens DC, Krishnan KR, Crump C, Burke GL. Cerebrovascular disease and evolution of depressive symptoms in the cardiovascular health study. Stroke. 2002;33:1636-1644.

17. Versluis CE, van der Mast RC, van Buchem MA, et al. Progression of cerebral white matter lesions is not associated with development of depressive symptoms in elderly subjects at risk of cardiovascular disease: The PROSPER Study. Int J Geriatr Psychiatry. 2006;21:375-381.

18. Harris TB, Launer L, Eiriksdottir G, et al. Age, Gene/Environment Susceptibility-Reykjavik Study: multidisciplinary applied phenomics. Am J Epidemiol. 2007;165:1076-1087.

19. Yesavage JA, Brink TL, Rose TL, et al. Development and validation of a geriatric depression screening scale: a preliminary report. J Psychiatr Res. 1982;17:37-49.

20. Almeida OP, Almeida SA. Short versions of the geriatric depression scale: a study of their validity for the diagnosis of a major depressive episode according to ICD-10 and DSM-IV. Int J Geriatr Psychiatry. 1999;14:858-865.

21. Scher AI, Gudmundsson LS, Sigurdsson S, et al. Migraine headache in middle age and late-life brain infarcts. JAMA. 2009;301:2563-2570.

22. Sveinbjornsdottir S, Sigurdsson S, Aspelund T, et al. Cerebral microbleeds in the population based AGES-Reykjavik study: prevalence and location. J Neurol Neurosurg Psychiatry. 2008;79:1002-1006.

23. Sigurdsson S, Aspelund T, Forsberg L, et al. Brain tissue volumes in the general population of the elderly: the AGES-Reykjavik study. Neurolmage. 2012;59:3862-3870.

24. Launer LJ, Hughes TM, White LR. Microinfarcts, brain atrophy, and cognitive function: the Honolulu Asia Aging Study Autopsy Study. Ann Neurol. 2011;70:774-780. 
25. Kloppenborg RP, Nederkoorn PJ, Grool AM, et al. Cerebral small-vessel disease and progression of brain atrophy: the SMART-MR study. Neurology. 2012;79:2029-2036.

26. Wardlaw JM, Smith C, Dichgans M. Mechanisms of sporadic cerebral small vessel disease: insights from neuroimaging. Lancet Neurol. 2013;12:483-497.

27. Bijanki KR, Stillman AN, Arndt S, et al. White matter fractional anisotropy is inversely related to anxious symptoms in older adults with atherosclerosis. Int J Geriatr Psychiatry. 2013;28:1069-1076.

28. Lambiase MJ, Kubzansky LD, Thurston RC. Prospective study of anxiety and incident stroke. Stroke. 2014;45:438-443.

29. Guralnik JM, Ferrucci L, Pieper CF, et al. Lower extremity function and subsequent disability: consistency across studies, predictive models, and value of gait speed alone compared with the short physical performance battery. J Gerontol A Biol Sci Med Sci. 2000;55:M221-231.

30. Vidal JS, Sigurdsson S, Jonsdottir MK, et al. Coronary artery calcium, brain function and structure: the AGES-Reykjavik Study. Stroke. 2010;41:891-897.

31. Schafer J. Analysis of Incomplete Multivariate Data. London: Chapman and Hall; 1997.

32. Schisterman EF, Cole SR, Platt RW. Overadjustment bias and unnecessary adjustment in epidemiologic studies. Epidemiology. 2009;20:488-495.

33. de Groot JC, de Leeuw FE, Oudkerk M, Hofman A, Jolles J, Breteler MM. Cerebral white matter lesions and depressive symptoms in elderly adults. Arch Gen Psychiatry. 2000;57:1071-1076.

34. Byers AL, Yaffe K. Depression and risk of developing dementia. Nat Rev Neurol. 2011;7:323-331.

35. Wechsler D. Wechsler Adult Intelligence Scale-Revised. Psychological Corporation, New York; 1988.

36. Kirton JW, Resnick SM, Davatzikos C, Kraut MA, Dotson VM. Depressive Symptoms, Symptom Dimensions, and White Matter Lesion Volume in Older Adults: A Longitudinal Study. Am J Geriatr Psychiatry. 2013. [Epub ahead of print].

37. Pan A, Sun Q, Okereke OI, Rexrode KM, Hu FB. Depression and risk of stroke morbidity and mortality: a meta-analysis and systematic review. JAMA. 2011;306:1241-1249.

38. Joynt KE, Whellan DJ, O'Connor CM. Depression and cardiovascular disease: mechanisms of interaction. Biol Psychiatry. 2003;54:248-261.

39. Ligthart SA, Richard E, Fransen NL, et al. Association of vascular factors with apathy in communitydwelling elderly individuals. Arch Gen Psychiatry. 2012;69:636-642.

40. Marin RS. Apathy: a neuropsychiatric syndrome. J Neuropsychiatry Clin Neurosci. 1991;3:243-254. 


\section{Supplemental Material}

Table S6.1. Associations between markers of baseline and progression of cerebral small vessel disease and incident depressive symptoms - depressive symptoms defined by a GDS-15 score $\geq 6$ ( $n=75$ )

\begin{tabular}{|c|c|c|}
\hline \multirow[t]{2}{*}{ Determinants } & \multicolumn{2}{|c|}{ Incident depressive symptoms } \\
\hline & $\beta$ coefficient $(95 \% \mathrm{Cl})$ & P-value \\
\hline \multicolumn{3}{|l|}{ White matter hyperintensity volume (\% ICV) } \\
\hline Per +1 SD volume at baseline & $1.04(0.80 ; 1.35)$ & .77 \\
\hline Per +1 SD volume change over time & $1.19(0.96 ; 1.47)$ & .11 \\
\hline \multicolumn{3}{|l|}{ Subcortical infarcts } \\
\hline Baseline presence of any infarct(s) vs. no infarcts & $1.62(0.74 ; 3.55)$ & .23 \\
\hline Any new infarct(s) over time vs. no new infarcts & $1.43(0.53 ; 3.83)$ & .50 \\
\hline \multicolumn{3}{|l|}{ Cerebral microbleeds } \\
\hline Baseline presence of any microbleed(s) vs. no microbleeds & $1.24(0.66 ; 2.32)$ & .50 \\
\hline Any new microbleed(s) over time vs. no new microbleeds & $1.98(1.11 ; 3.50)$ & .02 \\
\hline \multicolumn{3}{|l|}{ Virchow-Robin spaces } \\
\hline Baseline presence of any space(s) vs. no spaces & $1.40(0.76 ; 2.63)$ & .27 \\
\hline Any new space(s) over time vs. no new spaces & $5.81(2.19 ; 15.41)$ & $<.001$ \\
\hline \multicolumn{3}{|l|}{ Total brain parenchyma volume (\% ICV) } \\
\hline Per -1 SD volume at baseline & $1.10(0.84 ; 1.45)$ & .49 \\
\hline Per -1 SD volume change over time & $1.13(0.84 ; 1.51)$ & .42 \\
\hline
\end{tabular}

All associations are adjusted for baseline age, sex, Digit Symbol Substitution Test score, Mini Mental State Examination score, education level, presence of anxiety symptoms, gait speed, alcohol use, smoking, diabetes, body mass index, hypertension, coronary calcium score, head coil, follow-up time and baseline 15-item geriatric depression scale (GDS-15) score.

$\mathrm{Cl}$ : confidence interval; SD: standard deviation; ICV: intracranial volume.

Table S6.2. Associations between markers of baseline and progression over time of cerebral small vessel disease and incident depressive symptoms - individuals excluded with baseline stroke and/or incident stroke during follow-up ( $n=152)$

\begin{tabular}{lcc}
\hline Determinants & \multicolumn{2}{c}{ Incident depressive symptoms } \\
\cline { 2 - 3 } & $\beta$ coefficient $(95 \% \mathrm{Cl})$ & $\mathrm{P}$-value \\
\hline White matter hyperintensity volume $(\% \mathrm{ICV})$ & & .42 \\
$\quad$ Per +1 SD volume at baseline & $1.07(0.91 ; 1.27)$ & $<.05$ \\
$\quad$ Per +1 SD volume change over time & $1.18(1.01 ; 1.38)$ & .02 \\
Subcortical infarcts & & .06 \\
$\quad$ Baseline presence of any infarct(s) vs. no infarcts & $1.98(1.14 ; 3.45)$ & .46 \\
$\quad$ Any new infarct(s) over time vs. no new infarcts & $2.04(0.97 ; 4.29)$ & .36 \\
Cerebral microbleeds & & $.18(0.76 ; 1.82)$ \\
$\quad$ Baseline presence of any microbleed(s) vs. no microbleeds & $1.22(0.80 ; 1.86)$ & .88 \\
$\quad$ Any new microbleed(s) over time vs. no new microbleeds & & .001 \\
Virchow-Robin spaces & $0.97(0.61 ; 1.53)$ & $3.55(1.65 ; 7.67)$ \\
$\quad$ Baseline presence of any space(s) vs. no spaces & & .01 \\
$\quad$ Any new space(s) over time vs. no new spaces & $1.25(1.05 ; 1.50)$ & .008 \\
Total brain parenchyma volume $(\%$ ICV) & $1.33(1.08 ; 1.64)$ & \\
$\quad$ Per -1 SD volume at baseline & & \\
$\quad$ Per -1 SD volume change over time & & \\
\hline
\end{tabular}

All associations are adjusted for baseline age, sex, Digit Symbol Substitution Test score, Mini Mental State Examination score, education level, presence of anxiety symptoms, gait speed, alcohol use, smoking, diabetes, body mass index, hypertension, coronary calcium score, head coil, follow-up time and baseline 15-item geriatric depression scale (GDS-15) score.

Abbreviations as in Table S6.1. 
Table S6.3. Associations between presence of depressive symptoms at baseline (i.e. GDS-15 score of 6 or higher and/or use of antidepressant medication at baseline; $n=325$ ) and markers of progression over time of cerebral small vessel disease

\begin{tabular}{lcc}
\hline Outcome variables & $\beta$ coefficient $(95 \% \mathrm{Cl})$ & P-value \\
\hline +1 SD change of white matter hyperintensity volume over time (\% ICV) & $-0.01(-0.12 ; 0.12)$ & .94 \\
-1 SD change of total brain parenchyma volume over time (\% ICV) & $-0.06(-0.18 ; 0.06)$ & .30 \\
& Odds ratio $(95 \% \mathrm{Cl})$ & $\mathrm{P}$-value \\
\cline { 2 - 3 } Any incident subcortical infarct(s) vs. no incident infarcts & $1.47(0.81 ; 2.66)$ & .20 \\
Any incident cerebral microbleed(s) vs. no incident microbleeds & $1.37(0.99 ; 1.89)$ & .05 \\
Any incident Virchow-Robin space(s) vs. no incident spaces & $1.67(0.82 ; 3.42)$ & .16 \\
\hline
\end{tabular}

All associations are adjusted for baseline age, sex, Digit Symbol Substitution Test score, Mini Mental State Examination score, education level, presence of anxiety symptoms, gait speed, alcohol use, smoking, diabetes, body mass index, hypertension, coronary calcium score, head coil, follow-up time and corresponding marker of baseline cerebral small vessel disease.

Abbreviations as in Table S6.1. 


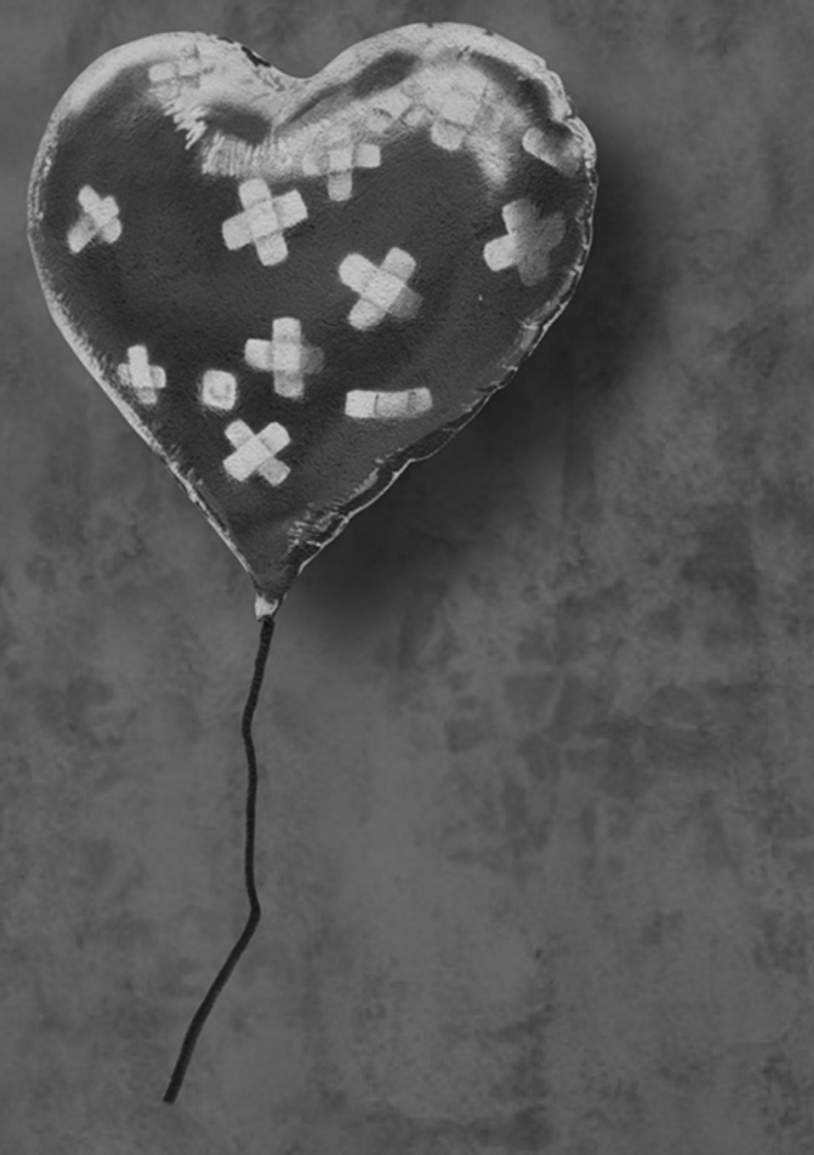

\section{9}




\section{Chapter 7}

\section{Arterial stiffness, depressive symptoms and mediation by cerebral small vessel disease: The AGES-Reykjavik Study}

TT van Sloten, GF Mitchell, S Sigurdsson, MA van Buchem, PV Jonsson, ME Garcia, TB Harris, RMA Henry, AS Levey, CDA Stehouwer, V Gudnason, 
158 | Chapter 7

\section{Abstract}

\section{Background}

Arterial stiffness may contribute to depression via cerebral microvascular damage. We investigated the association between arterial stiffness and depressive symptoms and the potential mediating role of cerebral small vessel disease therein.

\section{Methods}

Cross-sectional data of the AGES-Reykjavik study: $n=2,058$, mean age 79.6 years and $59.0 \%$ women. Arterial stiffness (carotid-femoral pulse wave velocity, cfPWV), depressive symptoms (15-item Geriatric Depression Score, GDS-15 score) and manifestations of cerebral small vessel disease (MRI) were determined.

\section{Results}

Higher cfPWV was associated with a higher GDS-15 score, after adjustment for potential confounders. Additional adjustment for white matter hyperintensity volume or subcortical infarcts attenuated the association between cfPWV and the GDS-15 score. The mediating effects of white matter hyperintensity volume and subcortical infarcts were statistically significant. Virchow-Robin spaces, microbleeds and cerebral atrophy did not mediate the association between cfPWV and depressive symptoms.

\section{Conclusion}

Arterial stiffness is associated with depressive symptoms; this association is partly mediated by white matter hyperintensity volume and subcortical infarcts. 


\section{Introduction}

Depression and depressive symptoms are frequently encountered in older individuals. ${ }^{1}$ Depressive symptoms in older populations, even in the absence of a clinical diagnosis of depression, are associated with a greatly increased morbidity and mortality risk. ${ }^{2,3}$ The pathobiology of late-life depression is incompletely understood, but it has been suggested that arterial stiffness is involved. ${ }^{4}$

Arterial stiffness impairs the cushioning function of large arteries, reduces wave reflection, and increases pressure and flow pulsatility, which transmits distally and damages the microcirculation.,6 Microvascular damage can manifest itself as cerebral small vessel disease, including white matter hyperintensities, subcortical infarcts, Virchow-Robin spaces, cerebral microbleeds and cerebral atrophy. ${ }^{7,8}$ Cerebral small vessel disease, in turn, may predispose to depression via disruption of frontal and subcortical structures involved in mood regulation..$^{9,10}$ In accordance, previous studies ${ }^{6,11-15}$ have shown an association between arterial stiffness and depression on the one hand and manifestations of cerebral small vessel disease on the other, independently of potential confounders (such as age, hypertension and other cardiovascular risk factors).

To date, only three studies ${ }^{4,16,17}$ have, however, evaluated the association between arterial stiffness and depression, and these studies showed inconsistent results. The Rotterdam Study ${ }^{4}$ and a small case-control study, ${ }^{16}$ which both included older individuals (mean age of both study samples 72 years), showed an association between higher arterial stiffness and presence of depression. In contrast, the Netherlands Study of Depression and Anxiety (NESDA) did not find an association between arterial stiffness and depression. ${ }^{17}$ However, NESDA included a relatively young study sample (mean age 47 years). Yet, it has been suggested that in particular in older individuals arterial stiffness and cerebral small vessel disease may contribute to depression. Importantly, these studies did not evaluate whether cerebral small vessel disease mediates the association (if any) between arterial stiffness and depression.

In view of the above, we investigated, in a large population-based cohort of older men and women, whether arterial stiffness is associated with depressive symptoms and whether any such association is mediated by manifestations of cerebral small vessel disease, including white matter hyperintensities, subcortical infarcts, Virchow-Robin spaces, cerebral microbleeds and cerebral atrophy. 


\section{Methods}

\section{Participants}

For the present study, we used cross-sectional data from the Age, Gene/Environment Susceptibility (AGES)-Reykjavik Study second examination (2007 to 2011). The AGESReykjavik Study is a population-based cohort study originating from the Reykjavik Study, as described fully elsewhere. ${ }^{18}$ This study aimed to investigate genetic and environmental factors and biological mechanisms leading to major clinical and subclinical disorders in old age, including those prevalent in neurocognitive, vascular, musculoskeletal, body compositional and metabolic symptoms. Briefly, from 2002 to 2006, 5,764 surviving participants in the Reykjavik Study were examined. From 2007 to 2011 there was a followup examination of all surviving participants who agreed to participate $(n=3,316)$. Reasons for not attending the follow-up examination included: death $(n=1,039)$; refusal $(n=1,198)$; and lost to follow-up (could not be contacted by any means) $(n=211)$. The AGES-Reykjavik Study was approved by the National Bioethics Committee in Iceland (approval number: VSN-00-063), and by the National Institute on Aging Intramural Institutional Review Board.

\section{Arterial stiffness}

Arterial stiffness was assessed by determining carotid-femoral pulse wave velocity (cfPWV), as previously described. ${ }^{6}$ Briefly, after 15-20 minutes of supine posture, brachial blood pressure was measured and arterial tonometry with electrocardiogram was obtained from the carotid and femoral arteries using a custom transducer (Cardiovascular Engineering, Inc., Norwood, MA). ${ }^{6}$ Transit distance from the carotid to femoral arteries was assessed by body surface measurements from the suprasternal notch to the carotid and femoral pulse recording sites. CfPWV was computed as the pulse wave transit distance divided by the transit time of the pulse wave from the carotid to femoral arteries, with adjustment for parallel transmission of the arterial pulse wave in the brachiocephalic artery and aortic arch. ${ }^{6}$

\section{Depressive symptoms}

Depressive symptoms were assessed by the 15-item Geriatric Depression Scale (GDS-15; score range, 0 to 15$).{ }^{19}$ Higher GDS-15 scores indicate the presence of more depressive symptoms. The GDS-15 score can be used as a continuous as well as a dichotomous variable. ${ }^{19}$ In the present study, we used the continuous variable as the primary outcome, because this offers the highest power to detect associations.

\section{Brain MRI measures}

Image acquisition. All eligible participants were offered a high-resolution brain MRI acquired on the same study-dedicated 1.5-T system (Signa Twinspeed, General Electric Medical Systems). The same imaging protocol was used as in the first examination of the 
AGES-Reykjavik Study, described elsewhere, ${ }^{18,20,21}$ and included the following sequences: 3D spoiled-gradient recalled T1-weighted, proton density/T2-weighted fast spin-echo, fluid-attenuated inversion recovery (FLAIR) and T2*-weighted gradient-echo type echoplanar (GRE-EPI). All images were acquired to give full brain coverage with slices angled parallel to the anterior commissure-posterior commissure line in order to give reproducible image views in the oblique-axial plane.

Image analysis. Several manifestations of cerebral small vessel disease were evaluated. White matter hyperintensity volume (WMHV) and total brain parenchyma volume (an indicator of cerebral atrophy) were computed automatically with a previously described image analysis pipeline, ${ }^{22}$ and were calculated as the percentage of total intracranial volume (ICV). Lower total brain parenchyma volume was considered to be a manifestation of cerebral small vessel disease, as cerebral small vessel disease leads to generalized loss of brain parenchyma via, amongst others, microinfarcts ${ }^{23}$ and loss of white matter integrity. ${ }^{24}$ Subcortical infarcts were evaluated as described previously, ${ }^{20}$ and defined as a brain parenchyma defect with a minimum diameter of $4 \mathrm{~mm}$, not extending into the cortex with a signal intensity equal to cerebrospinal fluid on all pulse sequences (i.e. T2weighted, proton density-weighted and FLAIR) and surrounded by an area of high signal intensity on FLAIR images. Parenchymal defects in the subcortical area with evidence of hemosiderin on the T2*-weighted GRE-EPI scan were labeled as resorbed hematomas and were excluded from the definition of subcortical infarcts. In addition, Virchow-Robin spaces were evaluated separately and defined as defects in the subcortical area without a rim or area of high signal intensity on FLAIR and without evidence of hemosiderin on the T2*-weighted GRE-EPI scan. Presence of Virchow-Robin spaces was considered to be a manifestation of cerebral small vessel disease, as they are associated with endothelial dysfunction, which may play a role in the pathogenesis of cerebral small vessel disease. ${ }^{7}$ Cerebral microbleeds were defined as a focal area of signal void within the brain parenchyma visible on T2*-weighted GRE-EPI scans and were identified as described previously. ${ }^{21}$

\section{Confounding variables}

Dementia (all sub-types) was diagnosed according to international guidelines ${ }^{25}$ by a panel that included a geriatrician, a neurologist, a neuropsychologist and a neuroradiologist as described elsewhere. ${ }^{18}$ Education (categorized into primary, secondary and college / university education) and smoking history (categorized into none, former and current smoker) were assessed by questionnaire. Gait speed, a measure of physical performance, ${ }^{26}$ was determined as the time in seconds needed to walk 6 meters at a usual pace. Diabetes mellitus was defined as a self-reported doctor's diagnosis of diabetes, use of blood glucose lowering drugs or fasting blood glucose level $\geq 7.0 \mathrm{mmol} / \mathrm{l}$. Coronary calcium score (categorized into sex specific quartiles), a measure of atherosclerosis, was 
based on Computed Tomography. ${ }^{27}$ The Digit Symbol Substitution Test (DSST), a measure of cognitive function, was also administered. ${ }^{18}$

\section{Analytic sample}

The final study sample consisted of 2,058 participants. Of the 3,316 participants of the second examination of the AGES-Reykjavik Study, 648 had missing brain MRI data, another 419 had missing data on arterial tonometry and another 77 had missing data on depressive symptoms. Missing MRI data was due to contraindications $(n=272)$, refusal/nonattendance $(n=337)$, or technical reasons (no qualitatively acceptable MRI data available for all necessary sequences, $n=39$ ). Missing data on tonometry was due to logistical or technical reasons. In the remaining 2,172 participants, 114 had a diagnosis of dementia and were excluded. Participants excluded for the present analysis were more likely to be older (81.2 vs. 76.6 years), less educated (for primary school or less: 23.6 vs. $18.9 \%)$ and to have a higher BMI (27.3 vs. $\left.26.5 \mathrm{~kg} / \mathrm{m}^{2}\right)$ and a higher prevalence of diabetes (17.6 vs. $11.6 \%)$ and/or stroke (11.5 vs. $8.2 \%)$ (P-value for all <.05).

\section{Statistical analysis}

Analyses were conducted with PASW statistics (version 21, IBM, Chicago, Illinois, USA). CfPWV was inverse-transformed to reduce heteroscedasticity and multiplied by $-1,000$ to restore directionality and to convert the units to milliseconds/meter. In addition, cfPWV was entered as a sex-specific Z-score in all models. WMHV was logarithmically transformed to normalize the skewed distribution and WMHV and lower total brain parenchyma volume were entered as Z-scores in all models.

We used mediation analysis to test the hypothesis that higher arterial stiffness and more depressive symptoms are associated, and that manifestations of cerebral small vessel disease (that is, higher WMHV, presence of subcortical infarcts, Virchow-Robin spaces and cerebral microbleeds and lower total brain parenchyma volume) are on the potential causal pathway of (i.e. mediate) the association between arterial stiffness and depressive symptoms. In mediation analysis, each of the above associations can be tested in one model. The model quantifies the degree to which a variable statistically mediates that is, changes, the association of a dependent variable with an independent variable. Specifically, it generates estimates of 1 ) the association of the independent with the dependent variable, 2 ) the association of the independent variable with the potential mediator, 3) the association of the potential mediator with the dependent variable, and 4) the proportion of the association between the independent and dependent variable that is attributed to the potential mediator (i.e. the mediated effect). We used bootstrapping $(10,000$ samples) to calculate bias-corrected $95 \%$ confidence intervals (Cls) of the mediated effects. In addition, the magnitude of the mediated effect was calculated as a percentage of the total direct effect. All models were adjusted for the following potential 
confounders: age, sex, education level, smoking, DSST, gait speed, body mass index, diabetes, mean arterial pressure, heart rate, coronary calcium score and the use of antihypertensive medication.

The following secondary analyses were done. Logistic analysis were done to evaluate the association between cfPWV and a dichotomous measure of depressive symptoms, defined as dichotomous GDS-15 score of 6 or higher ${ }^{19}$ or use of antidepressant medication. It has been suggested ${ }^{28}$ that cerebral small vessel disease may be associated with in particular apathy-/motivational-related symptoms of depression, and not with mood-related symptoms. Therefore, logistic regression analyses were done to evaluate the association between cfPWV and individual apathy items of the GDS-15 score as the outcome. The following three items of the GDS-15 score are related to apathy: 1) "Have you dropped many of your activities and interests?" (2) "Do you prefer to stay at home, rather than going out and doing new things?" and 3) "Do you feel full of energy?". ${ }^{28,29}$ As described previously, ${ }^{28,29}$ we distinguished an apathy subscale that included all apathy items (range 0-3; GDS-3A). Presence of apathy was defined as a GDS-3A score of 2 or 3 vs. no apathy (score of 0 or 1)..$^{28,29}$ To minimize the potential confounding effect of stroke, the analyses were repeated after excluding individuals with a clinical diagnosis of stroke. In addition, it has been suggested ${ }^{30}$ that deep or infratentorial cerebral microbleeds (i.e. microbleeds located in the basal ganglia, thalamus, brainstem and cerebellum) are more closely associated with vascular disease, whereas lobar microbleeds might be more closely associated with amyloid angiopathy. Therefore, analyses were repeated with presence of either deep or lobar cerebral microbleeds, instead of the presence of any microbleed. Finally, we investigated whether the association between arterial stiffness and depressive symptoms differed by sex by adding interaction terms between arterial stiffness and sex to the fully adjusted models. We found no such interaction (P-value for interaction=.57) and, therefore, all results are presented for men and women combined. 


\section{Results}

Characteristics of the study population are described in Table 7.1. Briefly, participants had a mean age of 79.6 years and $59.0 \%$ were women. Median cfPWV was $12.5 \mathrm{~m} / \mathrm{s}$ (interquartile range (IQR), 10.4 to 15.7 ), the median GDS-15 score was 2 (IQR 1 to 3), 5.3\% $(n=109)$ had a GDS-15 score of 6 or higher and $14.3 \%(n=294)$ used antidepressant medication.

Table 7.1. Characteristics of the study population $(n=2,058)$

\begin{tabular}{|c|c|}
\hline Variable & Value \\
\hline \multicolumn{2}{|l|}{ General characteristics } \\
\hline Age, years & $79.6 \pm 4.6$ \\
\hline Women, n (\%) & $1,214(59.0)$ \\
\hline \multicolumn{2}{|l|}{ Smoking status, n (\%) } \\
\hline Non-smoker & $897(43.6)$ \\
\hline Former & $983(47.8)$ \\
\hline Current & $177(8.6)$ \\
\hline \multicolumn{2}{|l|}{ Education, n (\%) } \\
\hline Primary or less & 398 (18.9) \\
\hline Secondary & $1,084(52.7)$ \\
\hline College/University & $584(28.4)$ \\
\hline Digit symbol substitution test score & $30 \pm 10$ \\
\hline Body mass index, $\mathrm{kg} / \mathrm{m}^{2}$ & $26.5 \pm 3.9$ \\
\hline Diabetes, n (\%) & $239(11.6)$ \\
\hline Stroke, $\mathrm{n}(\%)$ & $169(8.2)$ \\
\hline Systolic blood pressure, mmHg & $145 \pm 21$ \\
\hline Diastolic blood pressure, $\mathrm{mmHg}$ & $70 \pm 11$ \\
\hline Use of anti-hypertensive medication, $\mathrm{n}(\%)$ & $1,473(71.6)$ \\
\hline \multicolumn{2}{|l|}{ Depression measures } \\
\hline GDS-15 score & $2(1-3)$ \\
\hline GDS-15 score $\geq 6, \mathrm{n}(\%)$ & $109(5.3)$ \\
\hline Use of anti-depressant medication, n (\%) & $294(14.3)$ \\
\hline \multicolumn{2}{|l|}{ Arterial stiffness } \\
\hline Carotid-femoral pulse wave velocity, $\mathrm{m} / \mathrm{s}$ & $12.5(10.4-15.7)$ \\
\hline \multicolumn{2}{|l|}{ Brain MRI measures } \\
\hline White matter hyperintensity volume, ml & $15(8-28)$ \\
\hline Subcortical infarcts, n (\%) & $202(9.8)$ \\
\hline Virchow-Robin spaces, n (\%) & $364(17.7)$ \\
\hline Cerebral microbleeds, $\mathrm{n}(\%)$ & $603(29.3)$ \\
\hline Total brain parenchyma volume, $\mathrm{ml}$ & $1067 \pm 99$ \\
\hline
\end{tabular}

Data are presented as number (percentage) of participants, mean \pm standard deviation or median (interquartile range).

GDS-15 = 15-item geriatric depression scale.

\section{Association between arterial stiffness and depressive symptoms}

Higher cfPWV (per SD) was significantly associated with a higher GDS-15 score ( $\beta 0.096$ $(95 \% \mathrm{Cl} 0.005$ to 0.187$), \mathrm{P}=.039)$ ), after adjustment for confounders (but without adjustment for any of the potential mediators). 


\section{Mediation effects by manifestations of cerebral small vessel disease in the association between arterial stiffness and depressive symptoms}

Higher cfPWV (per SD) was significantly associated with a higher WMHV ( $\beta 0.050$ (0.002 to 0.097), $P=.039)$ ), and, in turn, higher WMHV (per SD) was significantly associated with a higher GDS-15 score ( $\beta 0.125$ (0.041 to 0.208), $P=.005)$ ) (Figure 7.1, panel A). When we adjusted the association between CFPWV and the GDS-15 score for WMHV, the association was attenuated and was no longer statistically significant ( $\beta 0.090$ (-0.001 to 0.181), $P=.053)$ ) (Figure 7.1, panel A). The mediated effect by WMHV was statistically significant ( $\beta 0.006$ (0.001 to 0.017$)$ ), and explained $6 \%$ of the total direct effect between cfPWV and the GDS-15 score (Table 7.2).

Similarly, higher cfPWV (per SD) was significantly associated with presence of subcortical infarcts (OR 1.27 (1.06 to 1.52), P=.013)) (Figure 7.1, panel B). Presence of subcortical infarcts, in turn, was significantly associated with a higher GDS-15 score ( $\beta 0.455$ (0.188 to 0.722), $\mathrm{P}=.001$ )) (Figure 7.1, panel $\mathrm{B}$ ). When we adjusted the association between cfPWV and the GDS-15 score for subcortical infarcts, the association was attenuated and was no longer statistically significant ( $\beta 0.088$ (-0.003 to 0.179$), P=.059)$ ) (Figure 7.1 , panel $B$ ). The mediated effect by subcortical infarcts was statistically significant ( $\beta \quad 0.008$ (0.002 to $0.020)$ ), and explained $9 \%$ of the total direct effect between cfPWV and the GDS-15 score (Table 7.2).

When WMHV and subcortical infarcts were entered simultaneously into the mediation model, they explained $12 \%$ of the total direct effect between cfPWV and the GDS-15 score.

In contrast, Virchow-Robin spaces, cerebral microbleeds and total brain parenchyma volume did not mediate the association between cfPWV and the GDS-15 score (Table 7.2 and supplemental material, Figure S7.1). Higher cfPWV (per SD) was associated with presence of Virchow-Robin spaces (OR 1.15 (1.00 to 1.32), $\mathrm{P}=.049$ ) and cerebral microbleeds (OR 1.09 (0.97 to 1.22), $\mathrm{P}=.151$ ), but these lesions, in turn, were not associated with depressive symptoms (for Virchow-Robin spaces: $\beta 0.04$ (-0.171 to 0.291), $\mathrm{P}=.763$; for cerebral microbleeds: $\beta 0.061$ (-0.114 to 0.236$), \mathrm{P}=.538)$. In addition, lower total brain parenchyma volume (per SD) was significantly associated with more depressive symptoms ( $\beta 0.124$ (0.031 to 0.218), $P=.009$ ), but cfPWV (per higher SD), in turn, was not associated with lower total brain parenchyma volume ( $\beta-0.012(-0.054$ to 0.031$), P=.587)$. 
A

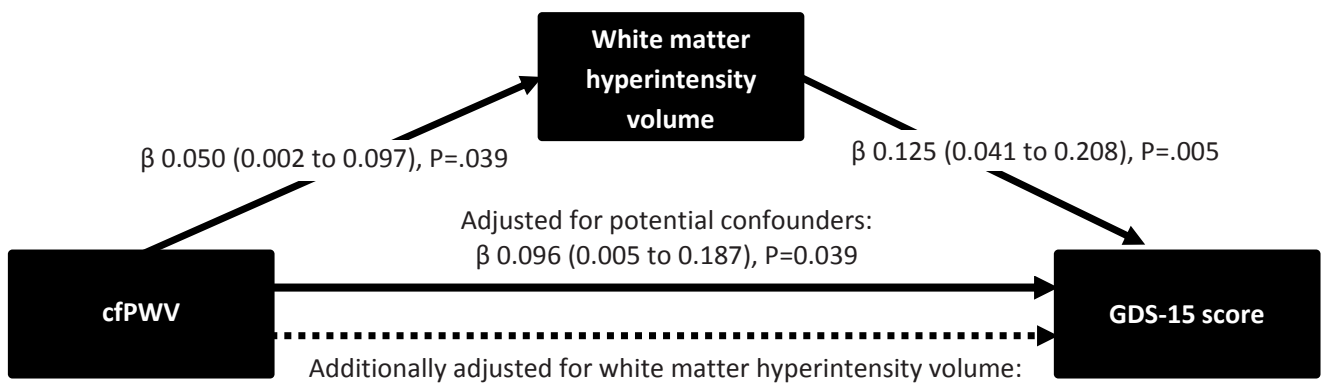

$\beta 0.090$ (-0.001 to 0.181$), P=.053$

B

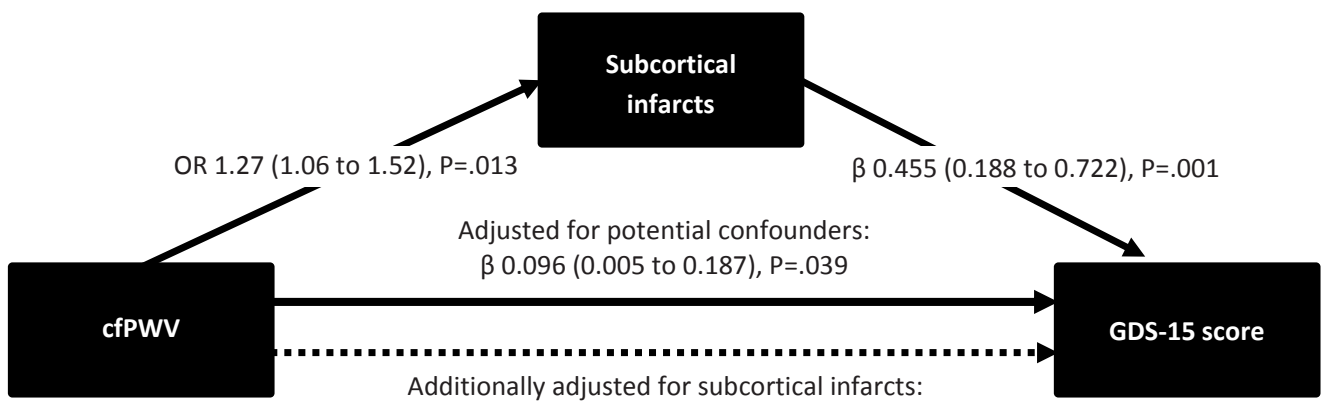

$\beta 0.088$ (-0.003 to 0.179), $P=.059$

Figure 7.1. Association between carotid-femoral pulse wave velocity (cfPWV) and 15-item Geriatric Depression Scale (GDS-15) score and the mediating effect of this association by white matter hyperintensity volume (panel A) and subcortical infarcts (panel B).

Solid lines indicate statistically significant associations; dashed lines indicate statistically not significant associations. Associations are given as regression coefficients $(\beta)$ or odds ratios (ORs), and corresponding $95 \%$ confidence intervals and P-values. CfPWV and white matter hyperintensity volume are indicated per higher standard deviation (SD). All associations are adjusted for age, sex, education level, smoking, Digit Symbol Substitution Test score, gait speed, body mass index, diabetes, mean arterial pressure, heart rate, coronary calcium score and use of anti-hypertensive medication.

Table 7.2. Mediating effects by white matter hyperintensity volume, subcortical infarcts, Virchow-Robin spaces, cerebral microbleeds and lower total brain parenchyma volume of the association between carotidfemoral pulse wave velocity and GDS-15 score

\begin{tabular}{lccc}
\hline & \multicolumn{3}{c}{ Mediated effects $^{\mathrm{A}}$} \\
\cline { 2 - 4 } Potential mediators & $\beta$ & $95 \% \mathrm{Cl}$ & $\%^{\mathrm{B}}$ \\
\hline White matter hyperintensity volume (per+1 SD) & 0.006 & $0.001 ; 0.017$ & $6 \%$ \\
Subcortical infarcts & 0.008 & $0.002 ; 0.020$ & $9 \%$ \\
Virchow-Robin spaces & 0.001 & $-0.003 ; 0.007$ & $1 \%$ \\
Cerebral microbleeds & 0.001 & $-0.000 ; 0.007$ & $1 \%$ \\
Lower total brain parenchyma volume (per -1 SD) & -0.002 & $-0.009 ; 0.003$ & $-2 \%$ \\
\hline
\end{tabular}

${ }^{A}$ All associations adjusted for age, sex, education level, smoking, digit symbol substitution test score, gait speed, body mass index, diabetes, mean arterial pressure, heart rate, coronary calcium score and use of antihypertensive medication.

${ }^{B}$ Percentages indicate the magnitude of the mediated effect relative to the total direct effect between carotidfemoral pulse wave velocity and GDS-15 score.

GDS-15 = 15-item geriatric depression scale; $\mathrm{Cl}=$ confidence interval; SD = standard deviation . 


\section{Secondary analyses}

CfPWV (per higher SD) was not significantly associated with a dichotomous measure of depressive symptoms (i.e. GDS-15 score of 6 or higher or use of antidepressant medication) (OR 1.10 (0.96 to 1.26), $\mathrm{P}=.183$ ). CfPWV (per higher SD) was not associated with apathy (i.e. GDS-3A score of 2 or 3 ) after adjustment for all potential confounders (OR 1.07 (95\%: 0.96 to 1.19, $\mathrm{P}=.237$ ). After the exclusion of individuals with stroke $(n=223)$, the mediation effects by WMHV and subcortical infarcts did not materially change (see supplemental material, Table S7.1). Finally, when we repeated the analyses with presence of either deep or lobar cerebral microbleeds, instead of the presence of any microbleed, results did not materially change (data not shown). 


\section{Discussion}

The present investigation evaluated the association between arterial stiffness and depressive symptoms and the potential mediating role of cerebral small vessel disease therein and had two main findings. First, arterial stiffness, as determined by cfPWV, was independently associated with a higher level of depressive symptoms. This association was independent of age, sex, education, cognitive function level, physical performance, blood pressure and cardiovascular risk factors. Second, this association was mediated in part by WMHV and subcortical infarcts, but not by Virchow-Robin spaces, cerebral microbleeds and cerebral atrophy. WMHV (per higher SD) and presence of subcortical infarcts together explained $12 \%$ of the total direct effect between cfPWV and depressive symptoms.

\section{Underlying mechanisms}

These data are consistent with the hypothesis that arterial stiffness leads to depression in part via cerebral microvascular damage. Arterial stiffness may cause microvascular damage via an increased pulsatile load on the microcirculation. ${ }^{31,32}$ This increased load causes direct microvascular damage and induces a microvascular remodeling response. Microvascular remodeling initially serves to limit the penetration of the pulsatile pressure load on the microcirculatory system by raising vascular resistance. Yet, this protective response may ultimately become unfavorable leading to impaired vasoreactivity and microvascular ischemia. ${ }^{31}$ Ischemia may damage frontal and subcortical structures or their connecting pathways involved in mood regulation and, hence, may lead to depression. ${ }^{9,10}$

In accordance, previous studies have shown an association between cfPWV or local carotid stiffness on the one hand and different manifestations of cerebral small vessel disease on the other, including $\mathrm{WMHV}^{6,11,15}$ and subcortical or lacunar infarcts. ${ }^{6,11,15}$ In addition, previous studies have shown an association between depression and WMHV ${ }^{12,13}$ and lacunar infarcts. ${ }^{13,14}$ Furthermore, one population-based study ${ }^{4}$ has shown that higher cfPWV and local carotid stiffness was associated with depression. The present study shows that the association between arterial stiffness and depressive symptoms is in part mediated by cerebral small vessel lesions and thereby provides additional evidence consistent with the role of arterial stiffness in modulating the emergence of late-life depressive symptoms.

However, a relatively large part of the association between arterial stiffness and depressive symptoms remained unexplained after taking into account the mediating effects of WMHV and subcortical infarcts. This remaining association may be due to vascular brain lesions not (directly) captured in the MRI scans (e.g. microinfarcts and loss of white matter integrity) that we did not incorporate into the mediation analyses. In 
addition, it is possible that only a subset of the persons doing poorly on the GDS-15 have vascular-related disease. Finally, although we adjusted for a large series of potential confounders, we cannot exclude the possibility of residual confounding.

Cerebral small vessel disease encompasses different lesions found on neuroimaging. ${ }^{6,7}$ Specific clinical consequences of each lesion type and their location are, however, not completely understood. In the present study, WMHV and subcortical infarcts mediated a part of the association between arterial stiffness and depressive symptoms, whereas Virchow-Robin spaces, cerebral microbleeds and cerebral atrophy did not. Although higher cfPWV was associated with presence of Virchow-Robin spaces and cerebral microbleeds, these lesions were not associated with depressive symptoms. Possibly, Virchow-Robin spaces and cerebral microbleeds play a role early in the pathogenesis of cerebral small vessel disease, ${ }^{33}$ whereas $\mathrm{WMHs}$ and subcortical infarcts represent more advanced disease states. The present study is, however, the first to explore the association between Virchow-Robin spaces and cerebral microbleeds on the one hand and depressive symptoms on the other, and further study is needed to clarify this issue. In addition, although lower brain parenchyma volume was associated with a higher level of depressive symptoms, brain parenchyma volume was not associated with cfPWV. Cerebral atrophy is strongly determined by factors other than vascular disease, in particular the process of neurodegeneration. ${ }^{7}$ This may have resulted in an underestimation of the association between cfPWV and brain parenchyma volume in the older population included in this study.

\section{Strengths and limitations}

Strengths of the present study are the large population-based sample of older individuals, the comprehensive brain MRI assessment of various manifestations of cerebral small vessel disease and the extensive characterization of participants which enabled us to adjust for a series of potential confounders.

Our study has some limitations. First, the cross-sectional design precludes any conclusions about a temporal association of arterial stiffness to cerebral small vessel disease and depressive symptoms. Second, depressive symptoms were assessed by the use of a selfreported questionnaire, but not by a structured interview. Therefore, no information was available on presence of a major depressive disorder. Third, it is possible that death or attrition between AGES-1 and -2 resulted in a disproportional loss of people who were likely at high risk for depression. If the associations among arterial stiffness, cerebral small vessel disease and depressive symptoms in these people are very different from our reported associations, the reported associations may be biased. However, we consider this unlikely, in which case the loss of the individuals reduces our power to detect existing associations. Fourth, the level and severity of depressive symptoms was relatively low in 
the present study sample, similar to previous studies done in Iceland. ${ }^{34}$ This low symptomatology may, however, have reduced the effect sizes and sensitivity to detect associations. This may explain why an association was found between arterial stiffness and a continuous depressive symptom score, but not with a dichotomous depression score. Analyses with a continuous outcome, in general, have higher statistical power than analyses with a dichotomous outcome. Finally, a relatively high number of individuals used antidepressant medication (14.3\%) as compared to the number of individuals with a GDS15 score of 6 or higher (5.3\%). Misclassification of the dichotomous depression score may have occurred because antidepressant medication is also prescribed for other reasons and this may have led to an underestimation of the observed associations.

\section{Clinical relevance}

In view of global ageing and the increased prevalence of arterial stiffness with age, efforts at favorably influencing arterial stiffness may have significant public health implications for preventing vascular-related depressions. Arterial stiffness may be favorably influenced by lifestyle modifications, such as weight loss, increased (habitual) physical activity and dietary modifications (e.g. low consumption of sodium). In addition, drugs, such as angiotensin receptor-2 agonists (e.g. compound 21) may lower arterial stiffness, possibly beyond any blood-pressure lowering effects. ${ }^{35}$

\section{Conclusion}

The present study shows that higher arterial stiffness and more depressive symptoms are associated in a general older population and that this association is in part mediated by WMHV and subcortical infarcts. 


\section{References}

1. Byers AL, Yaffe K, Covinsky KE, Friedman MB, Bruce ML. High occurrence of mood and anxiety disorders among older adults: The National Comorbidity Survey Replication. Arch Gen Psychiatry. 2010;67:489-496.

2. Barefoot JC, Schroll M. Symptoms of depression, acute myocardial infarction, and total mortality in a community sample. Circulation. 1996;93:1976-1980.

3. Pulska T, Pahkala K, Laippala P, Kivela SL. Follow up study of longstanding depression as predictor of mortality in elderly people living in the community. BMJ. 1999;318:432-433.

4. Tiemeier H, Breteler MM, van Popele NM, Hofman A, Witteman JC. Late-life depression is associated with arterial stiffness: a population-based study. J Am Geriatr Soc. 2003;51:1105-1110.

5. Mitchell GF, Parise H, Benjamin EJ, et al. Changes in arterial stiffness and wave reflection with advancing age in healthy men and women: the Framingham Heart Study. Hypertension. 2004;43:1239-1245.

6. Mitchell GF, van Buchem MA, Sigurdsson S, et al. Arterial stiffness, pressure and flow pulsatility and brain structure and function: the Age, Gene/Environment Susceptibility--Reykjavik study. Brain. 2011;134:3398-3407.

7. Wardlaw JM, Smith C, Dichgans M. Mechanisms of sporadic cerebral small vessel disease: insights from neuroimaging. Lancet Neurol. 2013;12:483-497.

8. Wardlaw JM, Smith EE, Biessels GJ, et al. Neuroimaging standards for research into small vessel disease and its contribution to ageing and neurodegeneration. Lancet Neurol. 2013;12:822-838.

9. Alexopoulos GS, Meyers BS, Young RC, Campbell S, Silbersweig D, Charlson M. 'Vascular depression' hypothesis. Arch Gen Psychiatry. 1997;54:915-922.

10. Krishnan KR, Hays JC, Blazer DG. MRI-defined vascular depression. Am J Psychiatry. 1997;154:497-501.

11. Brisset $\mathrm{M}$, Boutouyrie P, Pico F, et al. Large-vessel correlates of cerebral small-vessel disease. Neurology. 2013;80:662-669.

12. Godin O, Dufouil C, Maillard P, et al. White matter lesions as a predictor of depression in the elderly: the 3C-Dijon study. Biol Psychiatry. 2008;63:663-669.

13. Grool AM, Gerritsen L, Zuithoff NP, Mali WP, van der Graaf Y, Geerlings MI. Lacunar infarcts in deep white matter are associated with higher and more fluctuating depressive symptoms during three years follow-up. Biol Psychiatry. 2013;73:169-176.

14. Saavedra Perez HC, Direk N, Hofman A, Vernooij MW, Tiemeier H, Ikram MA. Silent brain infarcts: a cause of depression in the elderly? Psychiatry Res. 2013;211:180-182.

15. Tsao CW, Seshadri S, Beiser AS, et al. Relations of arterial stiffness and endothelial function to brain aging in the community. Neurology. 2013;81:984-991.

16. Paranthaman R, Greenstein AS, Burns AS, et al. Vascular function in older adults with depressive disorder. Biol Psychiatry. 2010;68:133-139.

17. Seldenrijk A, van Hout HP, van Marwijk HW, et al. Depression, anxiety, and arterial stiffness. Biol Psychiatry. 2011;69:795-803.

18. Harris TB, Launer LJ, Eiriksdottir G, et al. Age, Gene/Environment Susceptibility-Reykjavik Study: multidisciplinary applied phenomics. Am J Epidemiol. 2007;165:1076-1087.

19. Yesavage JA, Brink TL, Rose TL, et al. Development and validation of a geriatric depression screening scale: a preliminary report. J Psychiatric Res. 1982;17:37-49.

20. Scher Al, Gudmundsson LS, Sigurdsson S, et al. Migraine headache in middle age and late-life brain infarcts. JAMA. 2009;301:2563-2570.

21. Sveinbjornsdottir S, Sigurdsson S, Aspelund T, et al. Cerebral microbleeds in the population based AGES-Reykjavik study: prevalence and location. J Neurol Neurosurg Psychiatry. 2008;79:1002-1006.

22. Sigurdsson S, Aspelund T, Forsberg L, et al. Brain tissue volumes in the general population of the elderly: the AGES-Reykjavik study. Neurolmage. 2012;59:3862-3870. 
23. Launer LJ, Hughes TM, White LR. Microinfarcts, brain atrophy, and cognitive function: the Honolulu Asia Aging Study Autopsy Study. Ann Neurol. 2011;70:774-780.

24. Kloppenborg RP, Nederkoorn PJ, Grool AM, et al. Cerebral small-vessel disease and progression of brain atrophy: the SMART-MR study. Neurology. 2012;79:2029-2036.

25. American Psychiatric Association. Diagnostic and Statistical Manual of Mental Disorders, DMS-IV (4 ${ }^{\text {th }}$ edition). American Psychiatric Press, 1994.

26. Guralnik JM, Ferrucci L, Pieper CF, et al. Lower extremity function and subsequent disability: consistency across studies, predictive models, and value of gait speed alone compared with the short physical performance battery. J Gerontol A Biol Sci Med Sci. 2000;55:M221-231.

27. Vidal JS, Sigurdsson S, Jonsdottir MK, et al. Coronary artery calcium, brain function and structure: the AGES-Reykjavik Study. Stroke. 2010;41:891-897.

28. Ligthart SA, Richard E, Fransen NL, et al. Association of vascular factors with apathy in communitydwelling elderly individuals. Arch Gen Psychiatry. 2012;69:636-642.

29. Grool AM, Geerlings MI, Sigurdsson S, et al. Structural MRI correlates of apathy symptoms in older persons without dementia: AGES-Reykjavik Study. Neurology. 2014;82:1628-1635.

30. Greenberg SM, Vernooij MW, Cordonnier C, et al. Cerebral microbleeds: a guide to detection and interpretation. Lancet Neurol. 2009;8:165-174.

31. Mitchell GF. Effects of central arterial aging on the structure and function of the peripheral vasculature: implications for end-organ damage. J Appl Physiol (1985). 2008;105:1652-1660.

32. O'Rourke MF, Safar ME. Relationship between aortic stiffening and microvascular disease in brain and kidney: cause and logic of therapy. Hypertension. 2005;46:200-204.

33. Schreiber S, Bueche CZ, Garz C, et al. The pathologic cascade of cerebrovascular lesions in SHRSP: is erythrocyte accumulation an early phase? J Cereb Blood Flow Metab. 2012;32:278-290.

34. Copeland JR, Beekman AT, Braam AW, et al. Depression among older people in Europe: the EURODEP studies. World Psychiatry. 2004;3:45-49.

35. Mitchell GF. Arterial stiffness and hypertension. Hypertension. 2014;64:13-18. 


\section{Supplemental Material}

A

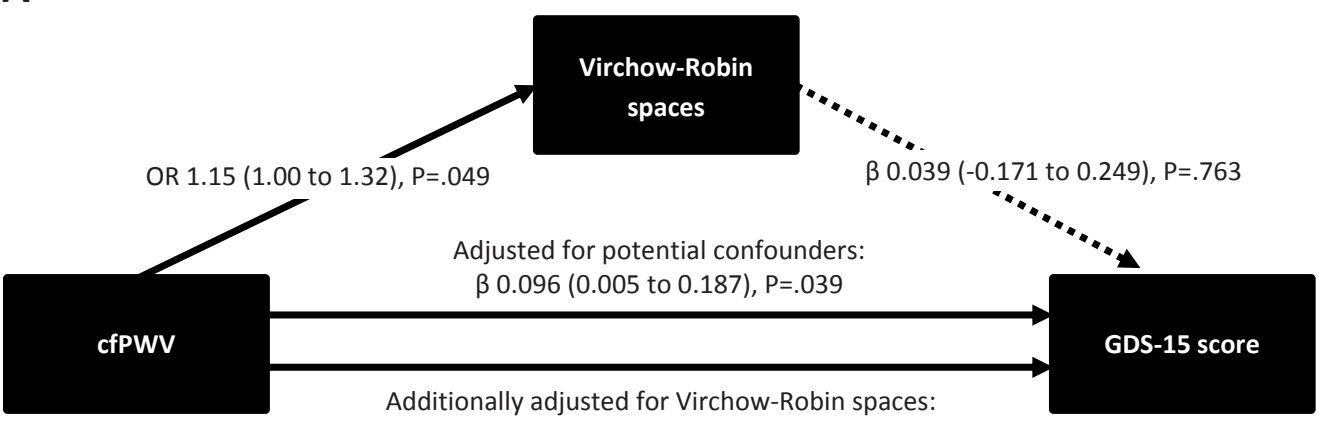

$\beta 0.096$ (0.004 to 0.188), $P=.041$

B

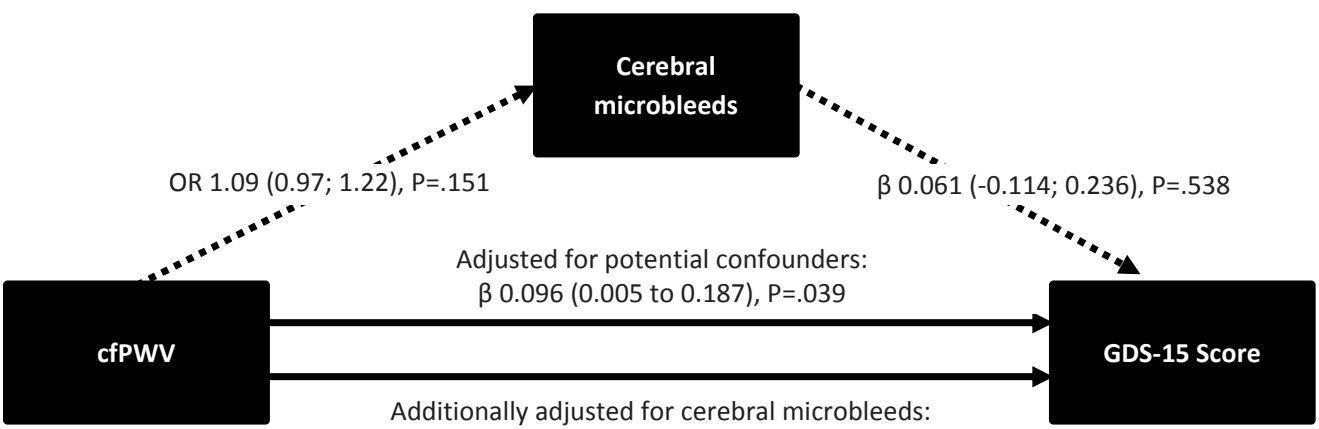

B 0.095 (0.004 to 0.187 ), $P=.041$

C

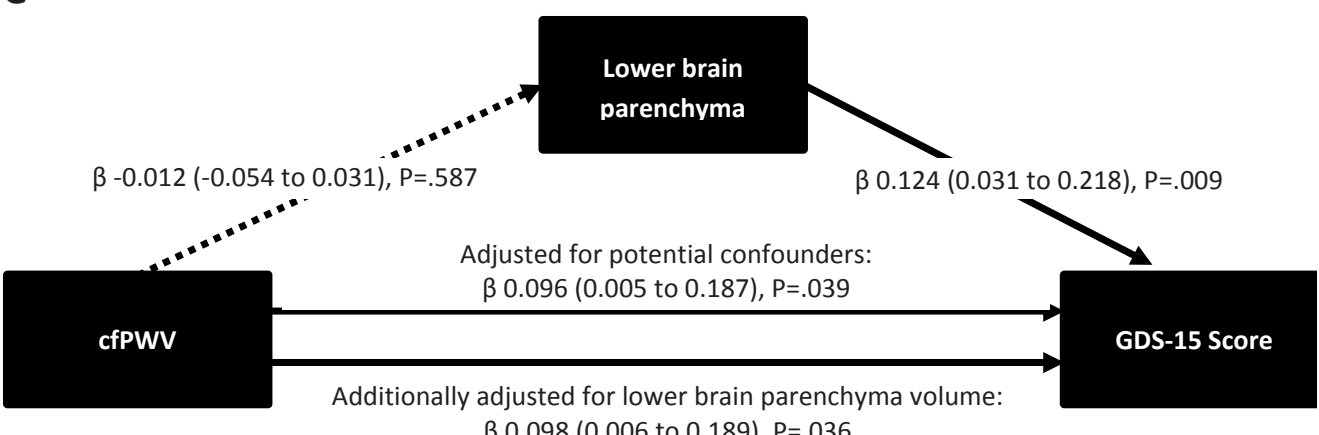

$\beta 0.098$ (0.006 to 0.189 ), $P=.036$

Figure S7.1. Association between carotid-femoral pulse wave velocity (cfPWV) and 15-item Geriatric Depression Scale (GDS-15) score and the mediating effects of this association by Virchow-Robin spaces (panel A), cerebral microbleeds (panel B) and lower total brain parenchyma volume (panel C).

Solid lines indicate statistically significant associations; dashed lines indicate statistically significant associations. Associations are given as regression coefficients ( $\beta$ ) or odds ratios (ORs), and corresponding $95 \%$ confidence intervals and P-values. CFPWV is indicated per higher standard deviation (SD) and total brain parenchyma volume per lower SD. All associations are adjusted for age, sex, education level, smoking, digit symbol substitution test score, gait speed, body mass index, diabetes, mean arterial pressure, heart rate, coronary calcium score and use of anti-hypertensive medication. 


\section{4 | Chapter 7}

Table S7.1. Mediating effects by white matter hyperintensity volume, subcortical infarcts, Virchow-Robin spaces, cerebral microbleeds and lower total brain parenchyma volume of the association between carotidfemoral pulse wave velocity and GDS-15 score - analyses done after exclusion of individuals with stroke $(n=223)$

\begin{tabular}{lccc}
\hline & \multicolumn{3}{c}{ Mediated effects $^{\mathrm{A}}$} \\
\cline { 2 - 4 } Potential mediators & $\beta$ & $95 \% \mathrm{Cl}$ & $\%^{\mathrm{B}}$ \\
\hline White matter hyperintensity volume (per +1 SD) & 0.005 & $-0.001 ; 0.016$ & $6 \%$ \\
Subcortical infarcts & 0.008 & $0.002 ; 0.020$ & $10 \%$ \\
Virchow-Robin spaces & 0.001 & $-0.004 ; 0.006$ & $1 \%$ \\
Cerebral microbleeds & 0.000 & $-0.002 ; 0.006$ & $1 \%$ \\
Lower total brain parenchyma volume (per -1 SD) & -0.001 & $-0.009 ; 0.003$ & $-1 \%$ \\
\hline
\end{tabular}

${ }^{\mathrm{A}}$ All associations adjusted for age, sex, education level, smoking, digit symbol substitution test score, gait speed, body mass index, diabetes, mean arterial pressure, heart rate, coronary calcium score and use of antihypertensive medication.

${ }^{B}$ Percentages indicate the magnitude of the mediated effect relative to the total direct effect between carotidfemoral pulse wave velocity and GDS-15 score.

GDS-15 = 15-item geriatric depression scale; $\mathrm{Cl}=$ confidence interval; SD = standard deviation. 
Arterial stiffness, depressive symptoms and cerebral small vessel disease | $\mathbf{1 7 5}$ 


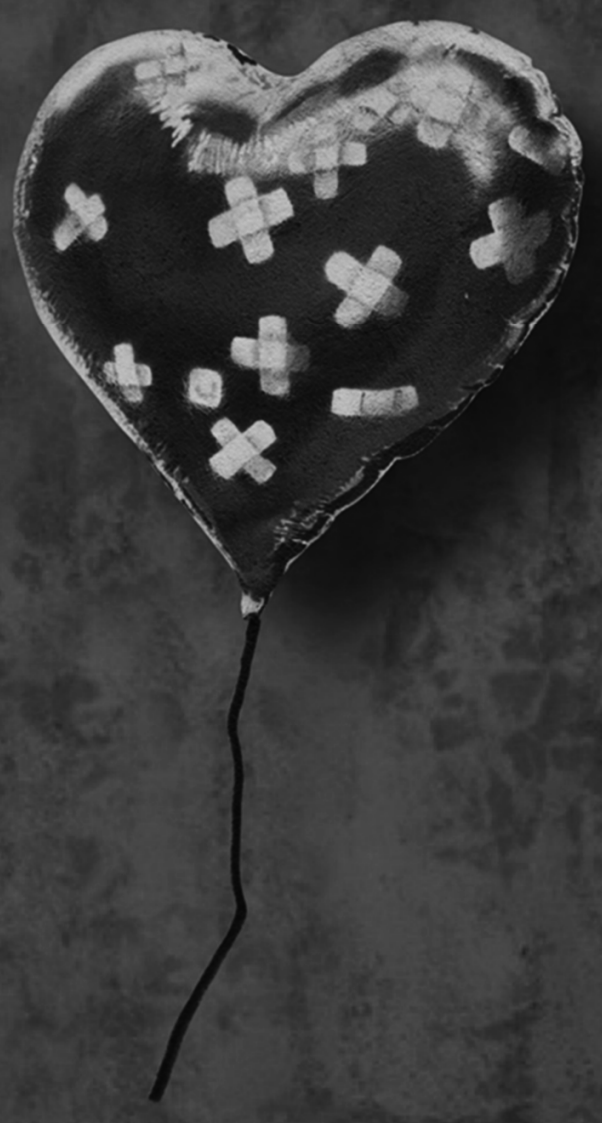

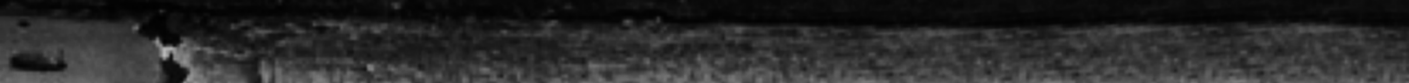
1. 1. 1. 


\section{Chapter 8}

\section{Endothelial dysfunction is associated}

with a greater depressive symptom score in a general elderly population: The Hoorn Study

TT van Sloten, MT Schram, MC Adriaanse, JM Dekker, G Nijpels, T Teerlink, PG Scheffer, F Pouwer, CG Schalkwijk, CDA Stehouwer, RMA Henry 
178 | Chapter 8

\begin{abstract}

\section{Background}

Endothelial dysfunction, inflammation and oxidative stress might be involved in the pathobiology of depression. Previous studies on the association of these processes in depression have yielded contradictory results. We therefore investigated comprehensively, in a population-based cohort study, the association between endothelial dysfunction, inflammation and oxidative stress on the one hand and depressive symptoms on the other.
\end{abstract}

\title{
Methods
}

For the present study, we used data from The Hoorn Study and determined biomarkers of endothelial dysfunction (flow-mediated dilatation [FMD], VWF, sICAM-1, sVCAM-1, sTM and sE-selectin), low-grade inflammation (CRP, TNF- $\alpha$, IL-6, IL-8, SAA, MPO and sICAM-1) and oxidative stress (oxLDL and MPO). Additionally, depressive symptoms were quantified by the CES-D questionnaire ( $n=493$; mean age 68 years; 49.9\% female). Regression analyses were performed with the use of biomarker Z-scores. Adjustments were made for age, sex and glucose metabolism status (cohort stratification variables), and prior cardiovascular disease, hypertension, waist-to-hip ratio, cholesterol levels, education level, physical activity, dietary habits, and the use of anti-hypertensive and/or lipidlowering medication and/or metformin (potential confounders).

\section{Results}

After adjustment for age, sex and glucose metabolism status, one standard deviation increase in the endothelial dysfunction Z-score was associated with a 1.9 (95\% confidence interval: 0.7 to 3.1) higher CES-D score. Additional adjustments did not materially change this result. Low-grade inflammation and oxidative stress were not associated with the CESD score.

\section{Conclusion}

Endothelial dysfunction, as quantified by an array of circulating biomarkers and FMD, was independently associated with depressive symptoms. This study supports the hypothesis that endothelial dysfunction plays an important role in the pathobiology of depression. 


\section{Introduction}

The pathobiology of depression is complex. It has been suggested that endothelial dysfunction (ED), low-grade inflammation (LGI) and oxidative stress (OxS) are involved, as these phenomena may interfere with neurotransmitter metabolism, the hypothalamicpituitary-adrenal (HPA) axis and the homeostatic process of neurogenesis in the brain. ${ }^{1-4}$ Yet, the above observations derive primarily from studies in animals. The study of these phenomena in the pathobiology of depression in humans is more complicated. One approach is to study ED, LGI and OxS via the determination of biomarkers in peripheral blood, which assumes that ED, LGI and OxS are generalized phenomena and that each of these phenomena represents either directly or indirectly ED, LGI, or OxS in the brain.

The concepts of $\mathrm{ED}^{5,6} \mathrm{LGI}^{4}$ and $\mathrm{OxS}^{7}$ are heterogeneous in nature. These concepts individually all can be defined in many different ways without it being clear that one definition necessarily favors over the other in relation to depression. For example, ED has been defined as brachial artery impaired flow-mediation, ${ }^{8}$ but also by an increased level of circulating biomarkers (e.g. soluble vascular cell adhesion molecule 1, soluble endothelial selectin, soluble thrombomodulin)..$^{9,10}$ In addition, many different circulating biomarkers have been used to assess LGI (e.g. C-reactive protein, interleukins 6 and 1, and tumor necrosis factor $\alpha)^{11,12}$ and OxS (e.g. myeloperoxidase and oxidized LDL). ${ }^{13,14}$ Furthermore, some studies ${ }^{8,15}$ used a single biomarker to define the concepts of ED, LGI and OxS, whereas others ${ }^{9,12}$ used multiple markers., ${ }^{9,12}$ The different definitions of the concepts of ED, LGI and OxS are exemplified by the fact that studies on the association between biomarkers of ED, ${ }^{10,16} \mathrm{LGI}^{12,15}$ and/or $\mathrm{OXS}^{13,17}$ and depression have yielded inconsistent results. ${ }^{8,9,14,18-26}$ In addition, these inconsistent results may be explained by the manner in which biomarkers of ED, LGI and/or OxS were determined (e.g. different laboratory techniques), the manner in which depression was assessed (e.g. interview vs. questionnaire) and the populations investigated (e.g. clinical- vs. population-based studies).

Nevertheless and taken together, many studies ${ }^{12,15,19,21,23}$ have found a positive association between LGI and depression. In particular for the LGI biomarkers C-reactive protein and the interleukins 6 and 1, most notably in clinical-based sampled studies and in studies in which depression was assessed by interview. ${ }^{11}$ For ED the evidence is less clear. In

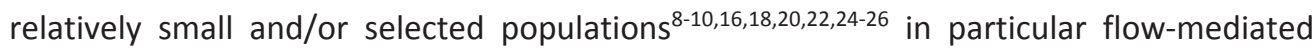
dilation was associated with depression to such an extent that a smaller FMD response was associated with more severe depressive symptoms. With regard to OxS, no clear picture emerges. Previous studies ${ }^{4,13,17}$ have defined OxS in many different ways and have yielded contradictory results. Important, however, is the fact that most of these studies 
examined ED, LGI and OxS in isolation whereas these processes are biologically interrelated and, therefore, may be interdependent. ${ }^{27}$

In view of these considerations, we investigated comprehensively, in a population-based study, the relationship between ED, LGI and OxS on the one hand and depressive symptoms on the other. In addition, we investigated whether any such associations were independent of diabetes, prior cardiovascular disease (CVD), physical activity, dietary habits and socio-economic status. Finally, we investigated whether ED, LGI and OxS were associated with depressive symptoms independently of each other.

\section{Methods}

\section{Study design}

For the present study, we used cross-sectional data from The 2000 Hoorn Study examination. The Hoorn Study is a population-based cohort study of glucose metabolism in relation to CVD risk factors, which started in 1989. ${ }^{28-30}$ Briefly, 2,484 men and women, aged 50 to 75 years, from the population register of the medium-sized Dutch town of Hoorn participated in the baseline examination. In 1996 to 1998 (visit 2), 1,513 (73\%) of al surviving participants agreed to participate in the first follow-up. In 2000 (visit 3), all those who were diagnosed as having diabetes during the previous examinations $(n=176)$, and random samples of individuals with normal glucose metabolism $(n=705)$ and impaired glucose metabolism $(n=193)$ were invited, of whom 648 (60\%) participated. The local ethics committee approved the study and all participants gave their written informed consent.

\section{Depressive symptoms}

Depressive symptoms were assessed by a validated Dutch version of the 20 -item Centre for Epidemiologic Studies Depression Scale (CES-D). ${ }^{31}$ Scores on the CES-D range from 0 to 60. Higher scores on this scale indicate the presence of more (severe) depressive symptoms. In the present study, the CES-D scale was used both as a continuous and as a dichotomous variable with a pre-defined cut-off level of $16 .{ }^{31}$ The latter represents the presence of clinically relevant depressive symptoms.

\section{ED, LGI and OxS}

ED was assessed by flow-mediated dilatation (FMD) of the brachial artery according to the guidelines of the International Brachial Artery Reactivity Task Force ${ }^{32}$ as previously described. ${ }^{33}$ In addition, ED was assessed by the quantification of the following circulating biomarkers: soluble vascular cell adhesion molecule 1 (sVCAM-1), soluble endothelial selectin (sE-selectin), soluble thrombomodulin (sTM), soluble intercellular adhesion 
molecule 1 (sICAM-1) and von Willebrand factor (vWf). Low-grade inflammation was assessed by the quantification of high-sensitivity C-reactive protein (CRP), serum amyloïd A (SAA), interleukin 6 (IL-6), interleukin 8 (IL-8), tumour necrosis factor $\alpha$ (TNF- $\alpha$ ), myeloperoxidase (MPO) and sICAM-1. Oxidative stress was determined by the quantification of oxidized LDL (oxLDL) and MPO.

Briefly, serum biomarkers of ED (sVCAM-1, sE-selectin, sTM, sICAM-1) and LGI (CRP, SAA, IL-6, IL-8, TNF- $\alpha$ ) were assessed by a multi-array detection system based on electrochemiluminescence technology (SECTOR Imager 2400, Meso Scale Discovery, Gaithsburg, Maryland, USA); details have been described elsewhere. ${ }^{34}$ In addition, vWf was determined in citrated plasma by means of ELISA, ${ }^{34}$ plasma OxLDL by competitive ELISA (Mercodia, Uppsala, Sweden) ${ }^{35}$ and MPO in EDTA plasma by a sandwich ELISA (Mercodia, Uppsala, Sweden). ${ }^{36}$

In our laboratory, intra- and inter-assay coefficients of variation (CV) were: for sVCAM-1, $2.8 \%$ and $5.6 \%$; for sE-selectin, $2.6 \%$ and $6.7 \%$; for STM, $2.1 \%$ and $6.9 \%$; for sICAM-1, $2.4 \%$ and $4.9 \%$; for CRP, $2.8 \%$ and $4.0 \%$; for SAA, $2.7 \%$ and $11.6 \%$; for IL-6, $5.6 \%$ and $13.0 \%$; for IL-8, $5.6 \%$ and $12.2 \%$ and for TNF- $\alpha, 3.9 \%$ and $8.8 \%$, respectively. In addition, the intraand inter-assay coefficients of variation were $3.4 \%$ and $7.9 \%$ for $\mathrm{vWf}^{34} ; 6.7 \%$ and $7.0 \%$ for OxLDL ${ }^{35}$; and $3.9 \%$ and $5.0 \%$ for $\mathrm{MPO},{ }^{36}$ respectively.

\section{Other measurements}

We determined medical history, education level, current medication use, anthropometrical (body height, weight, waist and hip circumference) and biological (blood pressure, total, high-density lipoprotein (HDL) and low-density lipoprotein (LDL) cholesterol, triglyceride and glucose levels, creatinine, albuminuria) variables as described elsewhere. ${ }^{28,30}$ For assessment of glucose status, all participants, except those with previously diagnosed diabetes, underwent a standard 75-g oral glucose tolerance test and were classified as having normal glucose metabolism (NGM), impaired glucose metabolism (IGM; impaired fasting glucose and/or impaired glucose tolerance), or type 2 diabetes, according to the 1999 World Health Organization criteria. ${ }^{37}$ Smoking habits were categorized as current, former and non-smokers. Hypertension was defined as a blood pressure $\geq 140 \mathrm{mmHg}$ systolic and/or $\geq 90 \mathrm{mmHg}$ diastolic and/or the current use of antihypertensive medication. Estimated glomerular filtration rate (eGFR) ( $\left.\mathrm{mL} / \mathrm{min} / 1.73 \mathrm{~m}^{2}\right)$ was calculated according to Levey's [Modification of Diet in Renal Disease, (MDRD)] short formula (without assay calibration) as follows: $186 \times$ (serum creatinine $)^{-1.154} \times(\text { age })^{-0.203} \times$ 1.212 (if black) x 0.742 (if female). ${ }^{38}$ Education level was dichotomized as low (secondary school or less) vs. higher education. Physical activity, expressed as Metabolic Equivalent (MET) hours per week, was assessed by the Short Questionnaire to Assess HealthEnhancing Physical Activity (SQUASH). ${ }^{39}$ Diet was assessed by a validated self-administered 
Food Frequency Questionnaire (FFQ). ${ }^{40-42}$ The FFQ queried participants to report habitual diet over the previous year. ${ }^{41,42}$ Based on the $F F Q$, we calculated the alternative Mediterranean (aMED) score as described by Fung et al. ${ }^{43}$ The aMED score quantifies "diet quality" and is based upon the dietary intake of vegetables, legumes, fruit, nuts, whole grains, meat, fish, unsaturated and saturated fat and ethanol.

\section{Statistical analysis}

All analyses were performed with PASW Statistics (version 18, IBM, Chicago, Illinois, USA). FMD was analysed as a functional marker of ED. For descriptive purposes, FMD values were reversed, i.e. multiplied by -1 (higher values indicating worse endothelial function) and a FMD Z-score was calculated according to the formula (individual value - population mean)/population standard deviation (SD). In all statistical analyses, the FMD Z-score was adjusted for baseline diameter, flow increase after cuff release and nitroglycerin-mediated dilation.

For reasons of statistical efficiency and to reduce the influence of the biological variability of each measure, a circulating biomarker Z-score was determined for the individual circulating biomarkers of ED, LGI and OxS according to predefined clusters of conceptually related biomarkers. ${ }^{44-46}$ The circulating biomarker Z-scores were calculated as follows: for each individual circulating biomarker, a Z-score was calculated. The resulting Z-scores were then averaged into the circulating biomarker Z-score for ED, LGI and OxS. The ED circulating biomarker Z-score consisted of sVCAM-1, sE-selectin, sTM, sICAM-1 and vWf. In addition, we combined the FMD Z-score and the ED circulating biomarker Z-score into one "total ED" score. The LGI circulating biomarker Z-score consisted of CRP, SAA, IL-6, IL-8, TNF- $\alpha$, MPO and sICAM-1. As both monocytes and the endothelium express SICAM- 1,47 sICAM-1 was included in the Z-score of both LGI and ED. The OxS circulating biomarker Zscore consisted of oxLDL and MPO. As MPO is both a measure of oxidative stress and inflammation, ${ }^{48}$ MPO was included in the Z-score of OxS and LGI. Linear and logistic regression analyses were used to evaluate the associations between, on the one hand, the total ED score, the FMD Z-score and the circulating biomarker Z-scores of ED, LGI and OxS, and, on the other, depressive symptoms (CES-D score; the analyses were done for both the continuous as well as the dichotomous CES-D score). We first adjusted, in all models, for the stratification variables of the Hoorn Study cohort: age, sex and glucose metabolism status (model 1). These associations were then additionally adjusted for the following sets of potential confounders ${ }^{16,20,23}$ : conventional cardiovascular disease risk factors (prior CVD, hypertension, waist-to-hip ratio (WHR), triglycerides, and total/HDL cholesterol (model 2)), lifestyle factors (education level, physical activity, smoking status, and aMED score (model 3)) and the use of anti-hypertensive and/or lipid-lowering medication and/or metformin (model 4). In the models 5 to 7, mutual adjustments were made for each of the individual Z-scores. 
The association between ED, LGI or OxS and depression might be different according to sex ${ }^{3,21}$ or glucose metabolism status. ${ }^{49}$ For instance, the hyperglycaemic state may amplify the effect of ED, LGI and/or OxS on depressive symptoms/depression, apart from the fact that the hyperglycaemic state itself enhances these processes. In addition, some 21,50 studies showed an association between LGI in men, but not in women. It has been speculated that this may be due to the effect of gonadal hormones on the level of plasma biomarkers. ${ }^{21}$ To investigate these possible interactions, we added, to our models, interaction terms between sex and ED, LGI and OxS, and between glucose metabolism status and ED, LGI and OxS.

A P-value $<.05$ was considered statistically significant, except for the interaction analyses, where P-values <.10 were used. Interaction analyses are handicapped in that they compare smaller subsets of study subjects and, thus, have less power than the primary study analysis. ${ }^{51}$ The use of a higher $\mathrm{P}$-value is therefore recommended ${ }^{52}$ to enable the detection of any potentially important interaction, despite the fact that such a greater Pvalue enhances the possibility of a type 1 error.

\section{Results}

\section{Participants}

Of the 648 participants, 84 had missing CES-D data and 14 had incomplete glucose data. In the remaining 550 participants, full data on circulating biomarkers of ED, LGI and OxS were available in 493 participants (study population), of which 357 had FMD measurements of sufficient quality (i.e. clear visual definition of the arterial wall throughout the entire measurement ${ }^{33}$ ). Participants with missing biomarker data were older (72 years vs. 69 years) and more often had type 2 diabetes (40\% vs. $20 \%$ ); P for all <.05. Participants with missing FMD data were older (72 years vs. 68 years), more often had type 2 diabetes (35\% vs. $17 \%$ ) and had a higher CES-D score (9 vs. 6); $\mathrm{P}$ for all <.05. In addition, these participants had a worse CVD risk factor pattern (data not shown).

\section{Clinical characteristics}

Tables 8.1 and 8.2 show the characteristics of the study population according to the presence of clinically relevant depressive symptoms (i.e. CES-D score $\geq 16$ ). According to the CES-D cut-off level, 63 participants (12.8\%) had clinically relevant depressive symptoms. In persons with clinically relevant depressive symptoms compared to those without, the total ED score, the FMD Z-score and the circulating biomarker Z-scores of ED, LGI and OxS were higher. 
Table 8.1. Clinical characteristics of the study population according to the presence of clinically important depressive symptoms (CES-D $\geq 16$ )

\begin{tabular}{|c|c|c|}
\hline & $\begin{array}{c}\text { CES-D score }<16 \\
n=430(87.2 \%)\end{array}$ & $\begin{array}{c}\text { CES-D score } \geq 16 \\
n=63(12.8 \%)\end{array}$ \\
\hline \multicolumn{3}{|l|}{ Demography } \\
\hline Age (years) & $68.0(64.0-74.0)$ & $71.0(66.0-75.0)$ \\
\hline Women & 48.1 & 61.9 \\
\hline \multicolumn{3}{|l|}{ Smoking status } \\
\hline Non-smoker & 20.9 & 36.5 \\
\hline Former smoker & 63.2 & 49.2 \\
\hline Current smoker & 15.9 & 14.3 \\
\hline Low education level & 21.4 & 36.5 \\
\hline Physical activity (MET hours/week) & $80(47-130)$ & $56(25-104)$ \\
\hline Alternative Mediterranean diet score & $4.0(3.0-5.0)$ & $4.0(3.0-5.0)$ \\
\hline \multicolumn{3}{|l|}{ Glucose metabolism status } \\
\hline Normal glucose metabolism & 49.8 & 30.2 \\
\hline Impaired glucose metabolism & 31.6 & 38.1 \\
\hline Type 2 diabetes mellitus & 18.6 & 30.1 \\
\hline Prior cardiovascular disease & 46.3 & 52.5 \\
\hline \multicolumn{3}{|l|}{ Metabolic variables } \\
\hline Body mass index $\left(\mathrm{kg} / \mathrm{m}^{2}\right)$ & $26.6(24.5-29.3)$ & $28.9(25.3-31.4)$ \\
\hline Waist-to-hip ratio & $0.93(0.86-0.99)$ & $0.94(0.86-0.99)$ \\
\hline Systolic blood pressure (mmHg) & $142(127-156)$ & $144(130-159)$ \\
\hline Diastolic blood pressure (mmHg) & $83(76-90)$ & $83(72-92)$ \\
\hline Hypertension & 67.9 & 71.4 \\
\hline $\mathrm{HbA1c}(\mathrm{mmol} / \mathrm{mol})$ & $39.9(37.7-44.3)$ & $41.0(37.0-47.0)$ \\
\hline $\mathrm{HbA1c}(\%)$ & $5.8(5.6-6.2)$ & $5.9(5.6-6.5)$ \\
\hline Total cholesterol (mmol/l) & $5.7(5.0-6.3)$ & $6.0(4.9-6.7)$ \\
\hline LDL cholesterol (mmol/l) & $3.6(3.0-4.2)$ & $3.8(2.9-4.4)$ \\
\hline HDL cholesterol (mmol/l) & $1.4(1.1-1.7)$ & $1.3(1.0-1.5)$ \\
\hline Triglycerides (mmol/l) & $1.3(1.0-1.7)$ & $1.4(1.2-2.1)$ \\
\hline Albuminuria (albumin/creatinine ratio $>2$ mg/mmol) & 14.7 & 9.5 \\
\hline Estimated Glomerular filtration rate $\left(\mathrm{ml} / \mathrm{min} / 1.73 \mathrm{~m}^{2}\right)$ & $77.4(70.0-89.5)$ & $76(64.8-89.7)$ \\
\hline \multicolumn{3}{|l|}{ Medication } \\
\hline Lipid-lowering medication & 15.6 & 17.5 \\
\hline Anti-hypertensive medication & 64.6 & 44.4 \\
\hline Anti-depressive medication & 1.9 & 3.2 \\
\hline
\end{tabular}

Values are $\%$, mean \pm standard deviation or median (interquartile range).

CES-D = center for epidemiological study of depression; $M E T=$ metabolic equivalent of task; $\mathrm{HbA} 1 \mathrm{c}=$ glycated hemoglobin; $L D L=$ low density lipoprotein; HDL = high density lipoprotein. 
Table 8.2. Markers of endothelial dysfunction, low-grade inflammation and oxidative stress according to the presence of clinically important depressive symptoms (CES-D $\geq 16$ )

\begin{tabular}{|c|c|c|}
\hline & $\begin{array}{c}\text { CES-D score }<16 \\
n=430(87.2 \%)\end{array}$ & $\begin{array}{c}\text { CES-D score } \geq 16 \\
n=63(12.8 \%)\end{array}$ \\
\hline \multicolumn{3}{|l|}{ Endothelial dysfunction } \\
\hline Total ED score & $-0.09(-0.42-0.29)$ & $0.15(-0.19-0.45)$ \\
\hline Flow-mediated dilatation Z-score (SD) & $0.09(-0.5-0.71)$ & $0.50(-0.18-0.91)$ \\
\hline Absolute change in diameter (mm) & $0.17(0.08-0.27)$ & $0.10(0.04-0.21)$ \\
\hline Percentage change in diameter (\%) & $3.7(1.5-5.9)$ & $2.5(0.7-4.8)$ \\
\hline Baseline diameter (mm) & $4.62(4.13-5.08)$ & $4.59(3.97-4.89)$ \\
\hline Flow increase after cuff release (\%) & $82(58-107)$ & $91(56-118)$ \\
\hline Nitroglycerin-mediated dilatation (mm) & $0.42(0.30-0.56)$ & $0.39(0.27-0.52)$ \\
\hline ED circulating biomarker Z-score (SD) & $-0.15(-0.44-0.30)$ & $0.04(-0.32-0.52)$ \\
\hline sVCAM-1 ( $\mu \mathrm{g} / \mathrm{l})$ & $390.6(342.3-446.4)$ & $427(350.4-486.1)$ \\
\hline sE-selectin ( $\mu \mathrm{g} / \mathrm{l})$ & $17.9(13.9-23.4)$ & $16.6(12.2-21.3)$ \\
\hline $\operatorname{sTM}(\mu \mathrm{g} / \mathrm{l})$ & $3.4(2.9-4.0)$ & $3.5(3.1-4.1)$ \\
\hline sICAM-1 ( $\mu \mathrm{g} / \mathrm{I})$ & $248.8(219.0-286.3)$ & $257.9(223.9-294.4)$ \\
\hline vWf (\%) & $146.5(114.9-180.0)$ & $177.4(135.2-212.0)$ \\
\hline \multicolumn{3}{|l|}{ Low-grade inflammation } \\
\hline LGI circulating biomarker Z-score (SD) & $-0.08(-0.43-0.26)$ & $0.10(-0.34-0.41)$ \\
\hline $\mathrm{CRP}(\mathrm{mg} / \mathrm{l})$ & $2.2(1.2-4.6)$ & $2.7(1.2-5.0)$ \\
\hline $\mathrm{SAA}(\mathrm{mg} / \mathrm{l})$ & $1.7(1.0-3.2)$ & $2.0(1.4-3.0)$ \\
\hline IL-6 (ng/l) & $1.5(1.1-2.4)$ & $1.8(1.2-2.6)$ \\
\hline IL-8 (ng/I) & $15.0(11.5-19.6)$ & $15.5(12.2-19.6)$ \\
\hline TNF- $\alpha(n g / l)$ & $8.4(7.1-10.0)$ & $8.9(7.8-10.3)$ \\
\hline MPO ( $\mu \mathrm{g} / \mathrm{I})$ & $55.2(46.9-65.2)$ & $57.3(48.8-67.6)$ \\
\hline sICAM-1 ( $\mu \mathrm{g} / \mathrm{I})$ & $248.8(219.0-286.3)$ & $257.9(223.9-294.4)$ \\
\hline \multicolumn{3}{|l|}{ Oxidative stress } \\
\hline OxS circulating biomarker Z-score (SD) & $-0.06(-0.48-0.37)$ & $0.18(-0.46-0.64)$ \\
\hline $\operatorname{oxLDL}(U / I)$ & $62.1(53.7-73.1)$ & $66.6(53.3-80.8)$ \\
\hline MPO ( $\mu \mathrm{g} / \mathrm{l})$ & $55.2(46.9-65.2)$ & $57.3(48.8-67.6)$ \\
\hline
\end{tabular}

Values are medians (interquartile range).

$\mathrm{SD}=$ standard deviation; CES-D = center for epidemiological study of depression; $E D$ = endothelial dysfunction; sVCAM-1 = soluble vascular adhesion molecule 1; sE-selectin: soluble endothelial selectin; sTM: soluble thrombomodulin; sICAM-1 = soluble intracellular adhesion molecule $1 ; \mathrm{vWf}=$ von Willebrand factor; $\mathrm{LGI}=\mathrm{low}-$ grade inflammation; CRP = C-reactive protein; SAA = serum amyloïd A; IL-6 = interleukin 6; IL-8 = interleukin 8; TNF- $\alpha=$ tumour necrosis factor $\alpha$; MPO = myeloperoxidase; OxS = oxidative stress; oxLDL = oxidized LDL.

\section{Association between ED, LGI and OxS on the one hand and depressive symptoms on the other}

The results of the linear regression analyses (CES-D expressed on a continuous scale) showed that, after adjustment for age, sex and glucose metabolism status, 1 SD increase in the total ED score was associated with a higher CES-D score with a regression coefficient of 1.9 (95\% confidence interval $(\mathrm{Cl})$ : 0.7 to 3.1 ) (Table 8.3, model 1 ; also illustrated in Figure 8.1, panel A). The LGI and OxS circulating biomarker Z-scores were not significantly associated with a higher CES-D score (regression coefficients: 0.4 (-0.6 to 1.5) and 0.7 (-0.1 to 1.5), respectively) (Table 8.3, models 1; also illustrated in Figure 8.1, panel A). Further adjustments for prior CVD, hypertension, WHR, total/HDL cholesterol, triglycerides, educational level, physical activity, smoking, aMED score and the use of anti-hypertensive and lipid-lowering medication, and metformin did not materially change these results (models 2 to 4). In addition, the associations for the total ED score and the OxS circulating 
biomarker Z-score did not materially change if model 1 was additionally adjusted for each of the other biomarkers scores (models 5 to 7). When we adjusted the LGI circulating biomarker Z-score for the ED circulating biomarker Z-score, the regression coefficient changed from 0.4 (-0.6 to 1.5$)$ to -0.1 (-1.2 to 1.1) (model 7). This change in the point estimate should nevertheless be interpreted with caution, as the confidence interval of both point estimates incorporates the critical value 0 .

\section{Association between ED, LGI and OxS on the one hand and clinically important depressive symptoms on the other}

The results of the logistic regression analyses (CES-D expressed on a dichotomous scale) showed that, after adjustment for age, sex and glucose metabolism status, 1 SD increase in the total ED score was associated with clinically important depressive symptoms with an odds ratio (OR) of 1.9 (0.9 to 3.8) (Table 8.4, model 1). The LGI and OxS circulating biomarker Z-scores were associated with clinically important depressive symptoms with an OR of 1.3 (0.8 to 2.0) and 1.2 (0.8 to 1.8 ), respectively (Table 8.4, model 1). Further adjustments for prior CVD, hypertension, WHR, total/HDL cholesterol, triglycerides, educational level, physical activity, smoking, aMED score and the use of anti-hypertensive and lipid-lowering medication, and metformin did not materially change these results (models 2 to 4). If we adjusted the total ED score for the LGI circulating biomarker Z-score, the OR changed from 1.9 (0.9 to 3.8) to 2.4 (1.1 to 5.4) (model 5). If we adjusted the results of the LGI and OxS circulating biomarker Z-scores for each of the other biomarker scores, results did not materially change (models 5 to 7 ). 


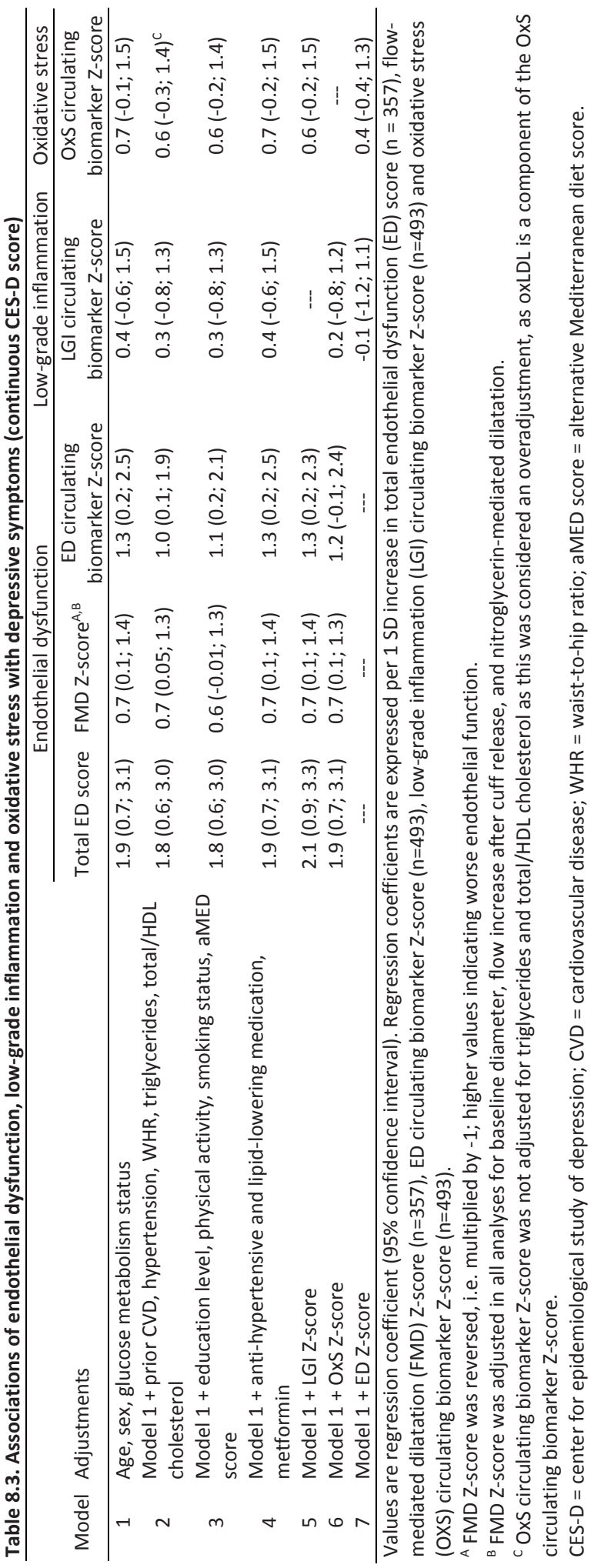




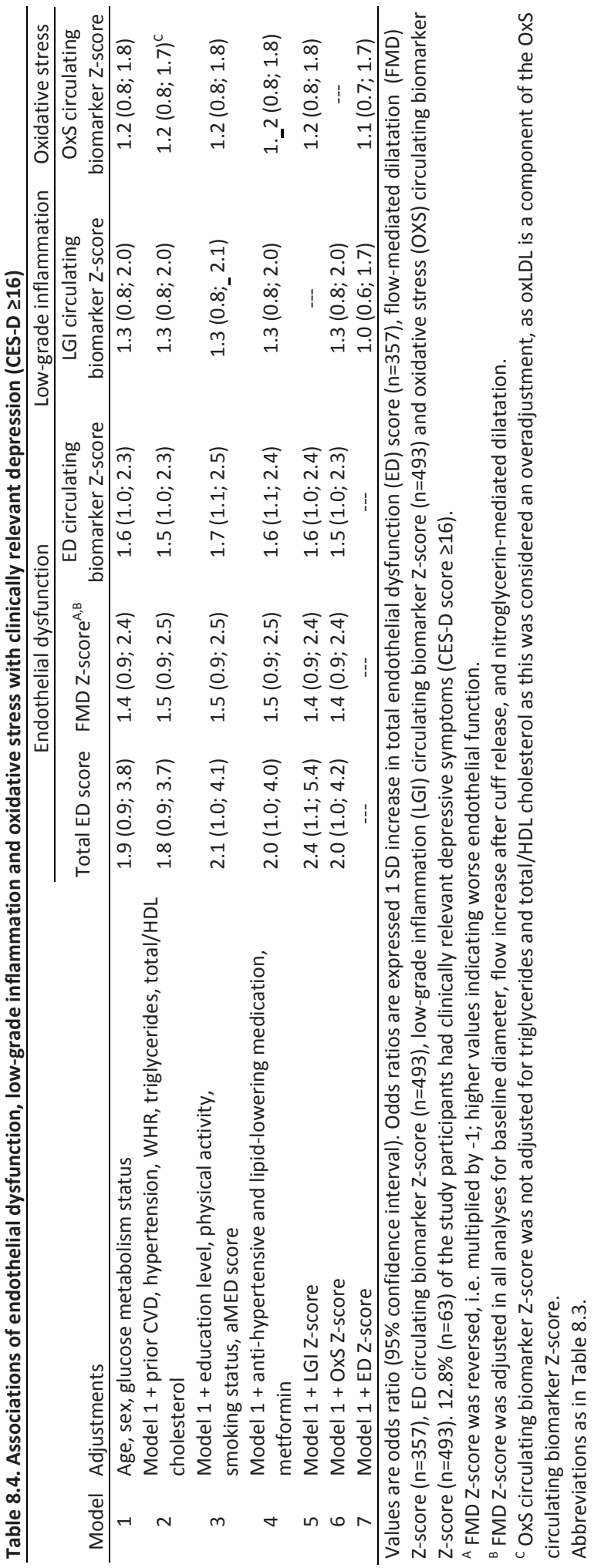




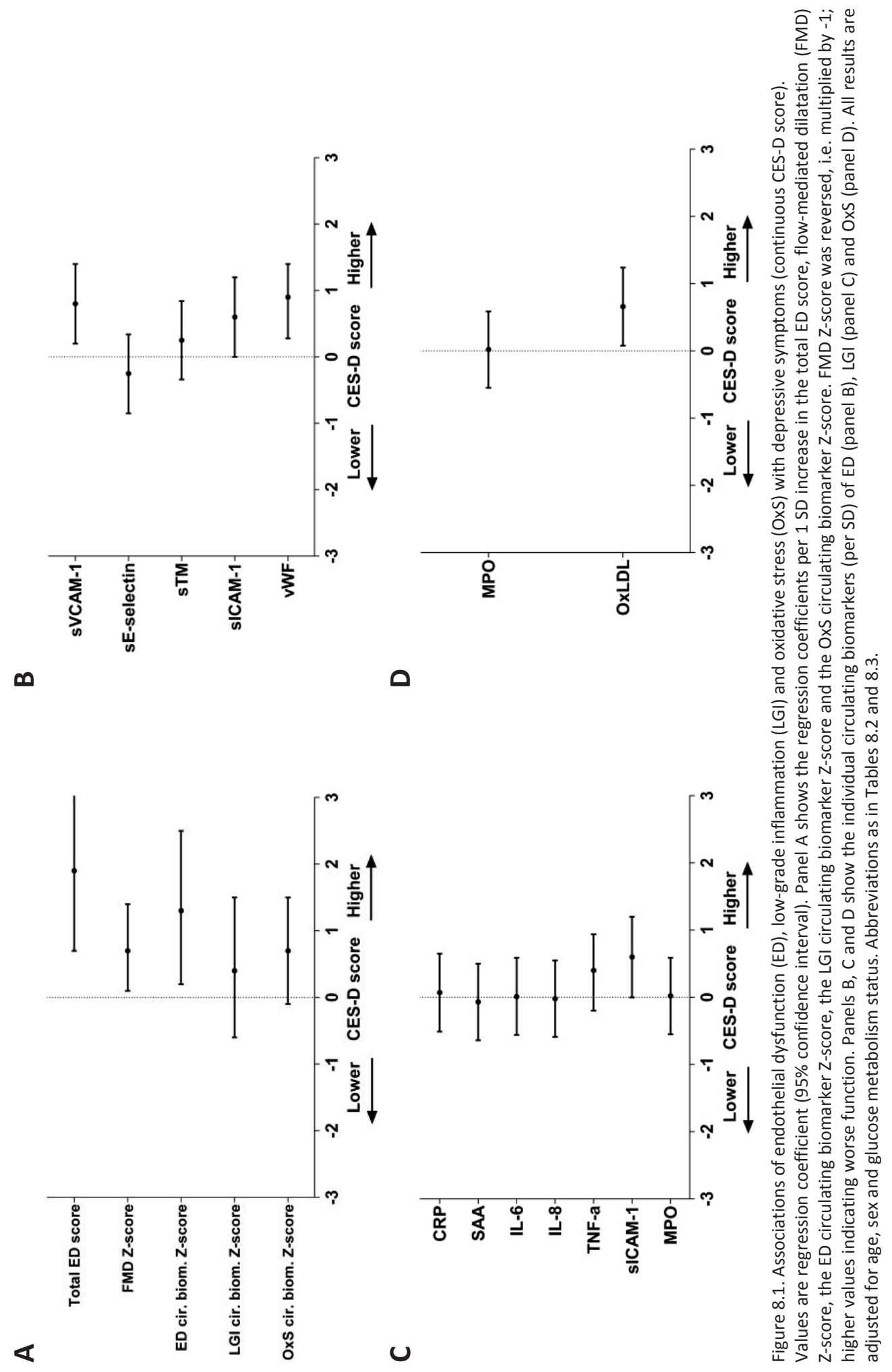




\section{Additional analyses}

Analyses of the associations between the individual elements of the ED, LGI and OxS circulating biomarker Z-scores and the CES-D score on a continuous scale showed that all individual circulating biomarkers, except sE-selectin and MPO, were associated with the CES-D score (statistically significant for sVCAM-1, vWf and oxLDL; this is illustrated in Figure 8.1, panels $B, C$ and D). Previous studies ${ }^{53,54}$ have suggested that sICAM-1 may be a marker of both ED and LGI. When the analyses were repeated with sICAM-1 left out of either the ED or the LGI circulating biomarker Z-score, results did not materially change (data not shown). In addition, we considered MPO a marker of both LGI and OxS. ${ }^{55,56}$ When the analyses were repeated leaving MPO out of the LGI circulating biomarker Zscore, results did not materially change (data not shown). Finally, it is unclear whether a high or a low concentration of sTM reflects ED. ${ }^{57}$ Results did not materially change when we performed the analysis with either the reversed value of STM or leaving STM out of the total ED score (data not shown).

To evaluate whether the association between FMD and depression was due to impaired endothelial function or smooth muscle cell function, ${ }^{33}$ we repeated the analyses with endothelium-independent nitroglycerine-mediated dilation (NMD) as the primary determinant. These analyses showed that NMD was not associated with (clinically relevant) depressive symptoms (data not shown).

The associations between ED, LGI and OxS on the one hand, and depressive symptoms on the other may differ according to sex or glucose metabolism status. ${ }^{3,49}$ Overall, we found no such interactions ( $P$ for interactions $>.10$ ), except that stratified analysis showed a stronger association between the LGI biomarker Z-score and depressive symptoms in persons with IGM as compared to persons with NGM ( $P$ for interaction $=.03$ ). In addition, the association between the OxS biomarker Z-score and depressive symptoms was stronger in persons with IGM ( $P$ for interaction $=.01$ ) and in persons with type 2 diabetes ( $P$ for interaction $=.07$ ), as compared to persons with NGM (data not shown).

When the statistical analyses were repeated on those participants $(n=357)$ who had both full data on circulating biomarkers and FMD, the results did not materially change (data not shown). Finally, when we repeated the analyses with clinically relevant depressive symptoms defined as a CES-D $\geq 16$ and/or medication use for a depressive disorder ( $n=67$, $13.6 \%)$ as the outcome variable instead of clinically relevant depressive symptoms only (CES-D $\geq 16$ ), the results did not materially change (data not shown). 


\section{Discussion}

The present investigation is the first population-based study that simultaneously assessed the association of ED, LGI and OxS with depressive symptoms in one study. The study had three main findings. Firstly, ED, as quantified by FMD and circulating biomarkers, was associated with a higher level of (clinically relevant) depressive symptoms. This association was independent of age, sex, diabetes, CVD risk factors, physical activity, dietary intake and education level. Secondly, circulating biomarkers for LGI and OxS were not statistically significantly associated with depressive symptoms. Thirdly, adjustments for LGI or OxS did not affect the association between ED and depressive symptoms, which suggests that ED is associated with depressive symptoms/depression, independently of LGI and OxS.

A key concept underlying this study is that ED, LGI and OxS are generalized phenomena and that each of these phenomena represents either directly or indirectly ED, LGI, or OxS in the brain. Currently, literature on this topic is limited. Nevertheless, it can be hypothesized that the impaired cerebral circulatory function seen in depression, as determined by transcranial Doppler ultrasonography, ${ }^{58}$ may be the consequence of decreased endothelium-dependent vasodilation. In addition, it has been shown that the process of neurogenesis (i.e. the process by which neural progenitors divide and form new neurons and neuronal networks) is disturbed in depression $n^{1,3}$ and that this disturbance may, at least partially, be the consequence of ED. ${ }^{59,60}$

On aggregate, most studies thus far $8,15,16,18,20,22,24-26$ have shown that ED and depression seem to be associated. However, conflicting results in the literature exist. For instance, Thomas et al. ${ }^{10}$ did not observe an association between ED and depression in a small and select population of patients diagnosed with a major depressive disorder. In this study, however, it is unclear how potential confounders were taken into account (e.g. antiinflammatory medication). An alternative explanation for the reported heterogeneous results may be the fact that endothelial function can be defined in many ways as its functions are multi-dimensional and heterogeneous. ${ }^{5,6}$ This is exemplified by Paranthaman et al. ${ }^{24}$ who did report an association between ED and depression, but quantified ED as the vasodilatory response to acetylcholine of biopsied small gluteal arteries. Do et al. ${ }^{9}$ quantified ED by multiple circulating biomarkers and reported an association between ED and, specifically, hopelessness, which reflects, as argued by the authors, a distinct and unique component of depression only. Pizzi et al. ${ }^{16}$ quantified ED by FMD and did show an association between FMD and depressive symptoms. However, in this study ${ }^{16}$ no adjustments were made for important confounders, such as physical activity and dietary habits. 
The present investigation extends previous observations because of its population-based design, the extensive assessment of ED, LGI and OxS, and the extensive clinical characterization of its participants, which enabled us to adjust for a series of potential confounders.

Apart from a causal association between ED and depression, other underlying mechanisms might explain the observed association. Firstly, ED is involved in the pathophysiology of CVD, 5,6 and depression is common in persons with CVD. ${ }^{1}$ Therefore, it is possible that ED might lead to depression via the development of CVD. However, the association of ED with depressive symptoms remained after adjustment for prior CVD and several CVD risk factors. Secondly, depressive symptoms by themselves might initiate or promote ED (reverse causality), as depressive symptoms/depression are associated with unfavourable lifestyle habits, such as physical inactivity, unhealthy dietary habits, smoking and obesity, which are by themselves associated with ED. The association between ED and depressive symptoms, however, remained after adjustment for unfavourable lifestyle habits. Thirdly, other mechanisms may underlie both ED as well as depression. For instance, abnormal HPA axis function ${ }^{61}$ and deficits in omega-3 fatty acids ${ }^{62}$ have been associated with both ED and depression. In the present study, however, cortisol and omega-3 fatty acids levels were not available and this issue needs further study. Finally, ED, LGI and OxS are, from a biological point of view, closely linked and these concepts are difficult to separate. ${ }^{27}$ Therefore, any association of ED with depressive symptoms may be confounded by LGI and/or OxS. However, when we adjusted the association between ED and depression for LGI or OxS, ED and depression remained associated. This suggests that ED may affect the brain via a pathway independent of LGI and OxS. For instance, it might be possible that ED directly affects the process of neurogenesis ${ }^{59,60}$ or alternatively, ED might directly impair cerebral circulatory function. ${ }^{63}$

In our study, the LGI and the OxS Z-score were associated with clinically relevant depressive symptoms with effect sizes comparable to the results reported in the literature. 4,15,17,20,21,23 However, these associations did not reach statistical significance in our study. We cannot exclude that this may be due to a lack of statistical power. In fact, a recent meta-analysis ${ }^{11}$ did show a significant association between different markers of LGI (CRP, IL-1 and IL-6) and depression/depressive symptoms. With regard to OxS in particular, we only assessed two biomarkers (MPO and oxLDL), in addition to the fact that there is an ongoing debate how OxS could best be defined.

We explored whether the relation between ED, LGI and OxS with depression differed according to glucose metabolism status. Our findings showed that, as compared to persons with NGM, the association between the LGI biomarker Z-score and depressive symptoms was stronger in persons with IGM. A plausible underlying pathobiological 
explanation for this observation is lacking and this finding might be the result of the play of chance. In addition, the results showed that the association between the OxS biomarker Z-score and depressive symptoms was stronger in persons with IGM and in persons with type 2 diabetes. One might speculate that the hyperglycaemic state may indeed amplify the effect of OxS on depressive symptoms/depression, apart from the fact that the hyperglycaemic state itself enhances OxS. Fully stratified analyses, however, were hampered by loss of power and further studies of this issue are needed.

Our study has some limitations. Firstly, the cross-sectional design of our study precludes any conclusions about causality and we cannot exclude that other factors may explain the association between ED and depressive symptoms/depression. Nevertheless, in our study, the association between ED and depressive symptoms remained after adjustment for glucose metabolism status CVD, obesity, physical inactivity, poor dietary habits, smoking and education level. Secondly, the construction of the Z-scores assumes that its components are equally important in the pathobiology of depression, which is not necessarily true. This might have caused us to underestimate the reported associations. However, the use of the composite Z-score has the important merit of statistical efficiency. Thirdly, nevertheless a relatively large number of statistical tests were done (we tested three ED scores, and one LGI and OxS score, respectively). The associations between ED and depressive symptoms, however, were consistent across the different ED scores. It is therefore unlikely that these findings result from the play of change. Finally, data were obtained in a white elderly population, and, therefore, it remains to be established whether these results can be generalized to other populations.

In conclusion, the present population-based study shows that ED, quantified by an array of peripherally circulating biomarkers and FMD, was associated with depressive symptoms. Our data thereby support the hypothesis that ED plays an important role in the pathobiology of depression. 


\section{References}

1. $\quad$ Belmaker RH, Agam G. Major depressive disorder. N Engl J Med. 2008;358:55-68.

2. Dantzer R, O'Connor JC, Freund GG, Johnson RW, Kelley KW. From inflammation to sickness and depression: when the immune system subjugates the brain. Nat Rev Neurosci. 2008;9:46-56.

3. Krishnan V, Nestler EJ. Linking molecules to mood: new insight into the biology of depression. Am J Psychiatry. 2010;167:1305-1320.

4. Miller AH, Maletic V, Raison CL. Inflammation and its discontents: the role of cytokines in the pathophysiology of major depression. Biol Psychiatry. 2009;65:732-741.

5. Aird WC. Phenotypic heterogeneity of the endothelium: II. Representative vascular beds. Circ Res. 2007;100:174-190.

6. Aird WC. Phenotypic heterogeneity of the endothelium: I. Structure, function, and mechanisms. Circ Res. 2007;100:158-173.

7. Maes $M$, Galecki $P$, Chang YS, Berk M. A review on the oxidative and nitrosative stress (O\&NS) pathways in major depression and their possible contribution to the (neuro)degenerative processes in that illness. Prog Neuropsychopharmacol Biol Psychiatry. 2011;35:676-692.

8. Sherwood A, Hinderliter AL, Watkins LL, Waugh RA, Blumenthal JA. Impaired endothelial function in coronary heart disease patients with depressive symptomatology. J Am Coll Cardiol. 2005;46:656-659.

9. Do DP, Dowd JB, Ranjit N, House JS, Kaplan GA. Hopelessness, depression, and early markers of endothelial dysfunction in U.S. adults. Psychosom Med.72:613-619.

10. Thomas AJ, Morris C, Davis S, Jackson E, Harrison R, O'Brien JT. Soluble cell adhesion molecules in latelife depression. Int Psychogeriatr. 2007;19:914-920.

11. Howren MB, Lamkin DM, Suls J. Associations of depression with C-reactive protein, IL-1, and IL-6: a meta-analysis. Psychosom Med. 2009;71:171-186.

12. Tiemeier H, Hofman A, van Tuijl HR, Kiliaan AJ, Meijer J, Breteler MM. Inflammatory proteins and depression in the elderly. Epidemiology. 2003;14:103-107.

13. Kupper N, Gidron Y, Winter J, Denollet J. Association between type D personality, depression, and oxidative stress in patients with chronic heart failure. Psychosom Med. 2009;71:973-980.

14. Maes M, Mihaylova I, Kubera M, Uytterhoeven M, Vrydags N, Bosmans E. Increased plasma peroxides and serum oxidized low density lipoprotein antibodies in major depression: markers that further explain the higher incidence of neurodegeneration and coronary artery disease. J Affect Disord. 2010;125:287-294.

15. Lesperance F, Frasure-Smith N, Theroux P, Irwin M. The association between major depression and levels of soluble intercellular adhesion molecule 1, interleukin-6, and C-reactive protein in patients with recent acute coronary syndromes. Am J Psychiatry. 2004;161:271-277.

16. Pizzi C, Manzoli L, Mancini S, Costa GM. Analysis of potential predictors of depression among coronary heart disease risk factors including heart rate variability, markers of inflammation, and endothelial function. Eur Heart J. 2008;29:1110-1117.

17. Forlenza MJ, Miller GE. Increased serum levels of 8-hydroxy-2'-deoxyguanosine in clinical depression. Psychosom Med. 2006;68:1-7.

18. Cooper DC, Milic MS, Tafur JR, et al. Adverse impact of mood on flow-mediated dilation. Psychosom Med.72:122-127.

19. Elovainio M, Aalto AM, Kivimaki M, et al. Depression and C-reactive protein: population-based Health 2000 Study. Psychosom Med. 2009;71:423-430.

20. Empana JP, Sykes DH, Luc G, et al. Contributions of depressive mood and circulating inflammatory markers to coronary heart disease in healthy European men: the Prospective Epidemiological Study of Myocardial Infarction (PRIME). Circulation. 2005;111:2299-2305.

21. Ford DE, Erlinger TP. Depression and C-reactive protein in US adults: data from the Third National Health and Nutrition Examination Survey. Arch Intern Med. 2004;164:1010-1014. 
22. Narita K, Murata T, Hamada T, et al. Interactions among higher trait anxiety, sympathetic activity, and endothelial function in the elderly. J Psychiatr Res. 2007;41:418-427.

23. Panagiotakos DB, Pitsavos C, Chrysohoou C, et al. Inflammation, coagulation, and depressive symptomatology in cardiovascular disease-free people; the ATTICA study. Eur Heart J. 2004;25:492499.

24. Paranthaman R, Greenstein AS, Burns AS, et al. Vascular function in older adults with depressive disorder. Biol Psychiatry.68:133-139.

25. Rybakowski JK, Wykretowicz A, Heymann-Szlachcinska A, Wysocki H. Impairment of endothelial function in unipolar and bipolar depression. Biol Psychiatry. 2006;60:889-891.

26. Schott LL, Kamarck TW, Matthews KA, Brockwell SE, Sutton-Tyrrell K. Is brachial artery flow-mediated dilation associated with negative affect? Int J Behav Med. 2009;16:241-247.

27. Stehouwer CD, Gall MA, Twisk JW, Knudsen E, Emeis JJ, Parving HH. Increased urinary albumin excretion, endothelial dysfunction, and chronic low-grade inflammation in type 2 diabetes: progressive, interrelated, and independently associated with risk of death. Diabetes. 2002;51:11571165.

28. Henry RM, Kostense PJ, Spijkerman AM, et al. Arterial stiffness increases with deteriorating glucose tolerance status: the Hoorn Study. Circulation. 2003;107:2089-2095.

29. Mooy JM, Grootenhuis PA, de Vries H, et al. Prevalence and determinants of glucose intolerance in a Dutch caucasian population. The Hoorn Study. Diabetes Care. 1995;18:1270-1273.

30. Spijkerman AM, Adriaanse MC, Dekker JM, et al. Diabetic patients detected by population-based stepwise screening already have a diabetic cardiovascular risk profile. Diabetes Care. 2002;25:17841789.

31. Beekman AT, Deeg DJ, Van Limbeek J, Braam AW, De Vries MZ, Van Tilburg W. Criterion validity of the Center for Epidemiologic Studies Depression scale (CES-D): results from a community-based sample of older subjects in The Netherlands. Psychol Med. 1997;27:231-235.

32. Corretti MC, Anderson TJ, Benjamin EJ, et al. Guidelines for the ultrasound assessment of endothelialdependent flow-mediated vasodilation of the brachial artery: a report of the International Brachial Artery Reactivity Task Force. J Am Coll Cardiol. 2002;39:257-265.

33. Henry RM, Ferreira I, Kostense PJ, et al. Type 2 diabetes is associated with impaired endotheliumdependent, flow-mediated dilation, but impaired glucose metabolism is not; The Hoorn Study. Atherosclerosis. 2004;174:49-56.

34. van Bussel BC, Henry RM, Schalkwijk CG, et al. Fish consumption in healthy adults is associated with decreased circulating biomarkers of endothelial dysfunction and inflammation during a 6-year followup. J Nutr. 2011;141:1719-1725.

35. van der Zwan LP, Teerlink T, Dekker JM, et al. Circulating oxidized LDL: determinants and association with brachial flow-mediated dilation. J Lipid Res. 2009;50:342-349.

36. Van der Zwan LP, Scheffer PG, Dekker JM, Stehouwer CD, Heine RJ, Teerlink T. Hyperglycemia and oxidative stress strengthen the association between myeloperoxidase and blood pressure. Hypertension. 2010;55:1366-1372.

37. Unwin N, Shaw J, Zimmet P, Alberti KG. Impaired glucose tolerance and impaired fasting glycaemia: the current status on definition and intervention. Diabet Med. 2002;19:708-723.

38. Levey AS, Coresh J, Greene T, et al. Expressing the Modification of Diet in Renal Disease Study equation for estimating glomerular filtration rate with standardized serum creatinine values. Clin Chem. 2007;53:766-772.

39. Wendel-Vos GC, Schuit AJ, Saris WH, Kromhout D. Reproducibility and relative validity of the short questionnaire to assess health-enhancing physical activity. J Clin Epidemiol. 2003;56:1163-1169.

40. $\mathrm{Du} \mathrm{H}$, van der $\mathrm{AD}$, van Bakel $\mathrm{MM}$, et al. Glycemic index and glycemic load in relation to food and nutrient intake and metabolic risk factors in a Dutch population. Am J Clin Nutr. 2008;87:655-661. 
41. Ocke MC, Bueno-de-Mesquita HB, Goddijn HE, et al. The Dutch EPIC food frequency questionnaire. I. Description of the questionnaire, and relative validity and reproducibility for food groups. Int $J$ Epidemiol. 1997;26 Suppl 1:S37-48.

42. Ocke MC, Bueno-de-Mesquita HB, Pols MA, Smit HA, van Staveren WA, Kromhout D. The Dutch EPIC food frequency questionnaire. II. Relative validity and reproducibility for nutrients. Int J Epidemiol. 1997;26 Suppl 1:S49-58.

43. Fung TT, McCullough ML, Newby PK, et al. Diet-quality scores and plasma concentrations of markers of inflammation and endothelial dysfunction. Am J Clin Nutr. 2005;82:163-173.

44. Jager A, van Hinsbergh VW, Kostense PJ, et al. von Willebrand factor, C-reactive protein, and 5-year mortality in diabetic and nondiabetic subjects: the Hoorn Study. Arterioscler Thromb Vasc Biol. 1999;19:3071-3078.

45. van Bussel BC, Schouten F, Henry RM, et al. Endothelial dysfunction and low-grade inflammation are associated with greater arterial stiffness over a 6-year period. Hypertension. 2011;58:588-595.

46. Yudkin JS, Stehouwer CD, Emeis JJ, Coppack SW. C-reactive protein in healthy subjects: associations with obesity, insulin resistance, and endothelial dysfunction: a potential role for cytokines originating from adipose tissue? Arterioscler Thromb Vasc Biol. 1999;19:972-978.

47. Schram MT, Stehouwer CD. Endothelial dysfunction, cellular adhesion molecules and the metabolic syndrome. Horm Metab Res. 2005;37 Suppl 1:49-55.

48. Schindhelm RK, van der Zwan LP, Teerlink T, Scheffer PG. Myeloperoxidase: a useful biomarker for cardiovascular disease risk stratification? Clin Chem. 2009;55:1462-1470.

49. Musselman DL, Betan E, Larsen H, Phillips LS. Relationship of depression to diabetes types 1 and 2: epidemiology, biology, and treatment. Biol Psychiatry. 2003;54:317-329.

50. Penninx BW, Kritchevsky SB, Yaffe K, et al. Inflammatory markers and depressed mood in older persons: results from the Health, Aging and Body Composition study. Biol Psychiatry. 2003;54:566572 .

51. Rothman KJ, Greenland S, Lash TJ. Modern Epidemiology. Philadelphia: Lippincott Williams And Wilkins; 2008.

52. Selvin S. Statistical analysis of epidemiologic data. Vol 3rd. New York: Oxford University Press Inc; 1996.

53. Brevetti G, Martone VD, de Cristofaro T, et al. High levels of adhesion molecules are associated with impaired endothelium-dependent vasodilation in patients with peripheral arterial disease. Thromb Haemost. 2001;85:63-66.

54. Ridker PM, Hennekens CH, Buring JE, Rifai N. C-reactive protein and other markers of inflammation in the prediction of cardiovascular disease in women. N Engl J Med. 2000;342:836-843.

55. van der Zwan LP, Teerlink T, Dekker JM, et al. Plasma myeloperoxidase is inversely associated with endothelium-dependent vasodilation in elderly subjects with abnormal glucose metabolism. Metabolism. 2010;59:1723-1729.

56. Brennan ML, Penn MS, Van Lente F, et al. Prognostic value of myeloperoxidase in patients with chest pain. N Engl J Med. 2003;349:1595-1604.

57. Wu KK. Soluble thrombomodulin and coronary heart disease. Curr Opin Lipidol. 2003;14:373-375.

58. Direk N, Koudstaal PJ, Hofman A, Ikram MA, Hoogendijk WJ, Tiemeier H. Cerebral Hemodynamics and Incident Depression: The Rotterdam Study. Biol psychiatry. 2012.

59. Shen Q, Goderie SK, Jin L, et al. Endothelial cells stimulate self-renewal and expand neurogenesis of neural stem cells. Science. 2004;304:1338-1340.

60. Zhao C, Deng W, Gage FH. Mechanisms and functional implications of adult neurogenesis. Cell. 2008;132:645-660.

61. Broadley AJ, Korszun A, Abdelaal E, et al. Metyrapone improves endothelial dysfunction in patients with treated depression. J Am Coll Cardiol. 2006;48:170-175.

62. Parker G, Gibson NA, Brotchie H, Heruc G, Rees AM, Hadzi-Pavlovic D. Omega-3 fatty acids and mood disorders. Am J Psychiatry. 2006;163:969-978. 
63. Lemke H, de Castro AG, Schlattmann P, Heuser I, Neu P. Cerebrovascular reactivity over time-course from major depressive episode to remission. J Psychiatr Res. 2010;44:132-136. 


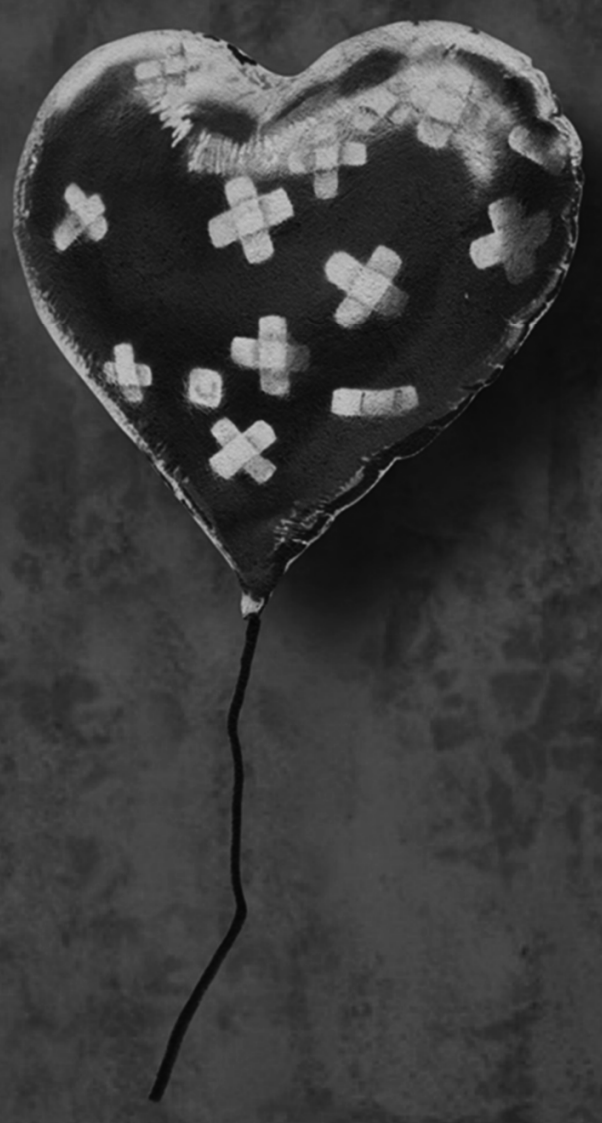

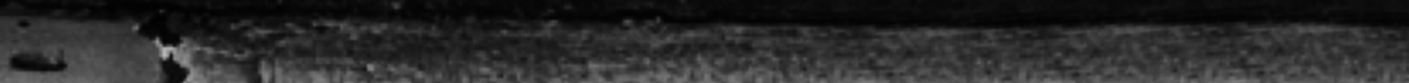
1. 1. 1. 


\section{Chapter 9}

\section{Association between arterial stiffness}

and skin microvascular function: The SUVIMAX2 Study and The Maastricht Study

TT van Sloten, S Czernichow, AJ Houben, AD Protogerou, RMA Henry, DM Muris, MT Schram, SJ Sep, PC Dagnelie, CJ van der Kallen, NC Schaper, J Blacher, S Hercberg, BI Levy, CDA Stehouwer Am J Hypertension - Accepted for publication 


\section{Abstract}

\section{Background}

It has been hypothesized that arterial stiffness leads to generalized microvascular dysfunction, and that individuals with type 2 diabetes (DM2) are particularly prone to the detrimental effects of arterial stiffness. However, evidence for an association between stiffness and markers of generalized microvascular dysfunction is lacking. We therefore investigated the association between arterial stiffness and skin microvascular function in individuals without and with DM2.

\section{Methods}

Cross-sectional data was used of The SUVIMAX2 Study ( $n=284$; mean age 62.2 years; 48.6\% women; 0\% DM2 (by design)) and The Maastricht Study ( $n=737$; mean age 59.7 years; $45.2 \%$ women; $28.8 \%$ DM2 (by design)). Arterial stiffness was determined by carotid-femoral pulse wave velocity (cfPWV). Skin capillaroscopy was used to determine capillary density at baseline, and during reactive hyperemia and venous congestion. Laser Doppler flowmetry was used to assess acetylcholine- and local heating-induced vasoreactivity, and skin flowmotion.

\section{Results}

In The SUVIMAX2 Study, cfPWV (per higher SD) was not associated with baseline capillary density (regression coefficient: -0.48 [95\% confidence interval: 2.37 to 1.41 ) or capillary recruitment during venous congestion $(0.54 \%[-0.74 ; 1.81 \%])$. In addition, cfPWV was not associated with acetylcholine $(-0.02 \%[-0.14$ to $0.10 \%])$ or local heating-induced vasoreactivity $(0.03 \%$ [ -0.07 to $0.12 \%]$ ). In The Maastricht Study, in individuals without DM2, cfPWV was not associated with baseline capillary density (-1.20 [-3.17 to 0.77]), and capillary recruitment during reactive hyperemia $(1.22 \%$ [-0.41 to $2.84 \%]$ ) or venous congestion (1.50\% [-0.25 to 3.25\%]). In addition, cfPWV was not associated with flowmotion (-0.01 [-0.07 to 0.06]). Results were adjusted for age and sex. Additional adjustments for confounders did not materially change these results. Results were qualitatively similar in individuals with DM2.

\section{Conclusion}

Arterial stiffness is not associated with skin microvascular function, irrespective of the presence of DM2. 


\section{Introduction}

Stiffening of large arteries impairs their cushioning function and increases pressure and flow pulsatility, which transmits distally and can damage the microcirculation. ${ }^{1,2}$ Indeed, previous studies have shown an association between greater arterial stiffness and markers of microvascular dysfunction in the brain (cerebral small vessel lesions ${ }^{3}$ ), eye (retinal arteriolar narrowing ${ }^{4}$ ) and kidney (microalbuminuria ${ }^{5}$ ). These organs are, however, especially vulnerable to the detrimental effects of increased pressure and flow pulsatility, as their microvasculature is characterized by low impedance, allowing the pulsatile load to penetrate deeply into their microvascular beds. ${ }^{1,2}$ Nevertheless, it has been hypothesized that increased arterial stiffness leads to generalized microvascular dysfunction, i.e. dysfunction not limited to microvascular beds characterized by low impedance. ${ }^{6}$ Such a phenomenon, if it exists, may explain the association between arterial stiffness and different diseases, including peripheral neuropathy, ${ }^{7}$ type 2 diabetes $(D M 2)^{8}$ and osteoporosis. ${ }^{9}$ Microvascular dysfunction is a common element in the pathophysiology of these diseases. ${ }^{6}$ However, evidence for an association between arterial stiffness and markers of generalized microvascular dysfunction is lacking.

The skin is a unique site which enables direct and non-invasive assessment of microvascular function both at rest and during provocative stimuli. Importantly, the skin microcirculation is considered a representative vascular bed to examine generalized microvascular phenomena. ${ }^{10,11}$ Skin microvascular dysfunction is associated with cardiovascular disease (CVD) risk factors, including, the metabolic syndrome, ${ }^{12} \mathrm{DM} 2{ }^{13}$ obesity $^{14-16}$ and hypertension. ${ }^{17}$ In addition, microvascular responses observed in skin parallel those in other tissues, including muscle. ${ }^{18,19}$ To date, only one small study $(n=76)^{20}$ has evaluated the association between arterial stiffness and skin microvascular function and did not find a significant association.

In view of the above, the aim of the present study was to evaluate the association between arterial stiffness, as determined by carotid-femoral pulse wave velocity (cfPWV), and skin microvascular function. Skin microvascular function was determined by baseline capillary density, capillary recruitment during reactive hyperemia after arterial occlusion and during venous congestion, endothelium-dependent and -independent skin vasoreactivity, and skin flowmotion. The associations were evaluated in two large studies: The Supplementation en Vitamines et Mineraux Antioxydants 2 (SUVIMAX2) Study and The Maastricht Study. In addition, it has been hypothesized that individuals with DM2 are particularly prone to the detrimental effects of increased pressure and flow pulsatility on the microcirculation, because DM2 may be associated with low microvascular impedance. ${ }^{1,21}$ We therefore additionally investigated whether any association between 
stiffness and microvascular function was stronger in individuals with DM2 as compared to those without DM2.

\section{Methods}

\section{Study design}

The present study used cross-sectional data of The SUVIMAX2 Study and The Maastricht Study.

The SUVIMAX Study $(n=12,749)$ was a prevention trial designed to investigate the effect of antioxidant supplementation on CVD and cancer, and was conducted in France between 1994 and 2002.22 In 2006 to 2007, 7,200 participants of the SUVIMAX trial participated in The SUVIMAX2 Study, an observational prospective cohort study on diet and aging. Of these, all individuals ( $n=291$ ) living in Paris without DM2, hypertension and prior CVD, underwent measurements on arterial stiffness and skin microvascular function. ${ }^{14}$

The Maastricht Study is an ongoing observational prospective population-based cohort study that aims to include 10,000 participants. The present study includes cross-sectional data from the first 866 participants, who completed the baseline survey between November 2010 and March 2012. The rationale and methodology have been described previously. ${ }^{23}$ In brief, the study focuses on the etiology, pathophysiology, complications and comorbidities of DM2 and is characterized by an extensive phenotyping approach. Eligible for participation were all individuals aged between 40 to 75 years and living in the southern part of the Netherlands. The study area is defined by postal codes. The study area encloses 82,462 inhabitants aged 40 to 75 years, including an estimated 7,000 individuals with DM2. Participants were recruited from the general population through mass media campaigns and from the municipal registries. In addition, individuals with DM2 were recruited through the regional Diabetes Patient Registry via mailings. Recruitment was stratified according to known DM2 status for reasons of efficiency. All examinations of each participant were performed within a time window of three months.

Both studies were approved by ethics committees: review committees of Paris-Cochin hospital (approval number: 2364) and the Comité National Informatique et des Libertés (approval number: 907094) for The SUVIMAX2 Study; and Maastricht University Medical centre (approval number: NL31329.068.10) and the Netherlands Health Council under the Dutch "Law for Population Studies" (approval number: 131088-105234-PG) for The Maastricht Study. All participants gave written informed consent. 
In both The SUVIMAX2 Study and The Maastricht Study, arterial stiffness was determined by cfPWV. In The SUVIMAX2 Study, microvascular function was assessed using skin capillaroscopy and endothelium-dependent and -independent skin vasoreactivity. In The Maastricht Study, microvascular function was assessed using skin capillaroscopy and skin flowmotion.

\section{Vascular measurements in The SUVIMAX2 Study}

All measurements were done by trained technicians unaware of the participants' clinical status in dark, quiet, temperature-controlled (21 to $24{ }^{\circ} \mathrm{C}$ ) rooms as described previously. ${ }^{14,24,25}$ Participants were asked to refrain from smoking and eating $\leq 12$ hours prior to the measurements. Measurements were done after 5 minutes of rest and talking or sleeping was not allowed during the examination.

\section{CFPWV}

CfPWV was determined with applanation tonometry (SphygmoCor, Atcor Medical, Sydney, Australia). ${ }^{24}$ Pressure waveforms were determined at the right common carotid and right common femoral arteries. Difference in the time of pulse arrival from the R-wave of the electrocardiogram between the two sites (transit time) was determined with the maximum upstroke algorithm. The pulse wave travel distance was calculated as the total direct straight distance (measured with an infantometer) between the two arterial sites. The mean of three consecutive cfPWV (defined as travel distance/transit time) recordings was used in the analysis.

\section{Skin capillaroscopy}

Capillaries were visualized in the dorsal skin of the middle phalanx of the dominant hand using a digital video microscope (Moritex, micro-ScopernanMS-500C, Tokyo, Japan; system magnification 200x). ${ }^{14,25}$ A region of interest of $3 \mathrm{~mm}^{2}$ skin area on the middle third of the phalanx was defined. Four microscopic fields of $1 \mathrm{~mm}^{2}$ were randomly chosen in this area. Capillary density (mean of four fields) was measured under two conditions. First, baseline capillary density was assessed. Second, capillary recruitment during venous congestion was determined, which is a measure of structural capillary reserve capacity. ${ }^{17}$ For venous congestion, a miniature cuff was applied on the base of the investigated finger and inflated to $50 \mathrm{mmHg}$ for 2 minutes. The number of perfused capillaries was counted manually using freeze-framed reproductions of the videotape by one investigator who was blinded to participants' clinical status. The intra- and inter-observer coefficient of variations (CVs) were $4.3 \%$ and $5.9 \%$, respectively, as described previously. ${ }^{20}$

\section{Endothelium-dependent and-independent skin vasoreactivity}

Endothelium-dependent and -independent skin vasoreactivity were determined by acetylcholine iontophoresis and local skin heating, respectively. ${ }^{14}$ Skin blood perfusion was 
measured using a laser Doppler system (Periflux 5000, Perimed, Stockholm, Sweden), equipped with a thermostatic laser probe (PF 457, Perimed) at the dorsal side of the wrist. Skin temperature was monitored continuously and maintained at $33^{\circ} \mathrm{C}$. Baseline skin blood perfusion was defined as the mean value during a time period of 4 minutes. Acetylcholine chloride ( $2 \%$ solution, $800 \mu \mathrm{l}$ ) was delivered on the dorsal side of the wrist using an anodal current (three doses of 10 milliamps for 10 seconds with a 2 minutes interval) and maximal increase in blood perfusion was measured. Next, the laser probe was heated to $44^{\circ} \mathrm{C}$ for 5 minutes and maximal increase in blood perfusion was measured.

\section{Vascular measurements in The Maastricht Study}

All measurements were done by trained technicians unaware of the participants' clinical status, in dark, quiet, temperature-controlled (21 to $24{ }^{\circ} \mathrm{C}$ ) rooms as described previously. ${ }^{10,23}$ Participants were asked to refrain from smoking and drinking coffee, tea or alcoholic beverages $\leq 3$ hours prior to the study. Participants were allowed to have a light meal (breakfast and/or lunch). All measurements were performed after 10 minutes of rest and talking or sleeping was not allowed during the examination.

\section{CfPWV}

CfPWV was determined according to recent guidelines ${ }^{26}$ with applanation tonometry (SphygmoCor, Atcor Medical). Pressure waveforms were determined at the right common carotid and right common femoral arteries. Transit time was determined with the intersecting tangents algorithm. The pulse wave travel distance was calculated as $80 \%$ of the direct straight distance (measured with an infantometer) between the two arterial sites. The median of three consecutive cfPWV recordings was used in the analysis. Reproducibility was assessed in 12 individuals ( 6 men; $60.8 \pm 6.8$ years; 6 DM2) who were examined by two observers at two occasions spaced one week apart. The intra- and interobserver CVs were $13.5 \%$ and $16.2 \%$, respectively.

\section{Skin capillaroscopy}

Capillaries were visualized in the dorsal skin of the distal phalanges of the third and fourth finger of the right hand using a digital video microscope (Capiscope ${ }^{\circledR}$, KK Technology, Honiton, UK; system magnification $100 \mathrm{x}) .{ }^{10}$ Capillaries were visualized $4.5 \mathrm{~mm}$ proximal to the terminal row of capillaries in the middle of the nailfold. The investigator selected a region of interest of $1 \mathrm{~mm}^{2}$ skin area. Capillary density (mean of two fields) was measured under three conditions. First, baseline capillary density was measured. Second, capillary recruitment during reactive hyperemia was assessed after 4 minutes of arterial occlusion. Capillary recruitment during reactive hyperemia reflects functional and/or structural capillary reserve capacity. ${ }^{17}$ For the assessment of reactive hyperemia, a miniature cuff was applied on the base of the investigated finger and inflated to suprasystolic pressure (260 $\mathrm{mmHg}$ ) for 4 minutes. Third, venous congestion was applied, with the cuff inflated to 
$60 \mathrm{mmHg}$ for 2 minutes. The number of continuously perfused capillaries was counted using a semi-automatic procedure (CapiAna) and running movie files by two investigators who were blinded to participants' clinical status. ${ }^{10}$ The intra- and inter-observer CVs of CapiAna were $2.5 \%$ and $5.6 \%$, respectively, as described previously. ${ }^{10}$

\section{Skin flowmotion}

Skin flowmotion, i.e. blood flow fluctuation attributed to the rhythmic contraction of arterioles, is an important component of the microcirculation. ${ }^{16}$ It is involved in optimal delivery of nutrients to tissue and regulation of local vascular resistance. ${ }^{16,27}$ To assess flowmotion, skin blood perfusion was measured using a laser Doppler system (Periflux 5000, Perimed), equipped with a thermostatic laser probe (PF 457, Perimed) at the dorsal side of the wrist. Skin temperature was monitored continuously and maintained at $30^{\circ} \mathrm{C}$. The Doppler flowmetry output was recorded for 25 minutes (sample rate $32 \mathrm{~Hz}$ ). FastFourier Transform algorithm was performed using Perisoft dedicated software (PSW version 2.50) to measure the power density of the flowmetry oscillation. The frequency spectrum between 0.01-1.6 Hz was divided into five flowmotion components ${ }^{28}: 1$ ) endothelial, 0.01-0.02 Hz; 2) neurogenic, 0.02-0.06 Hz; 3) myogenic, 0.06-0.15 Hz; 4) respiratory, $0.15-0.40 \mathrm{~Hz}$; and 5 ) heartbeat, $0.40-1.60 \mathrm{~Hz}$. In addition, total flowmotion energy was obtained by the sum of the power density values of the total frequency spectrum.

\section{Potential confounders}

CVD risk factors were determined as described previously in The SUVIMAX2 Study ${ }^{14,24,25}$ and The Maastricht Study. ${ }^{23}$

\section{Statistical analysis}

All analyses were performed with PASW Statistics (version 21, IBM, Chicago, Illinois, USA). Separate analyses were done for The SUVIMAX2 Study and The Maastricht Study. In addition, because The Maastricht Study population is enriched with individuals with DM2, results are presented separately for individuals without and with DM2. Microvascular recruitment during reactive hyperemia after arterial occlusion and during venous congestion was expressed as percentage change in capillary density from baseline. Additional analyses were done using absolute recruitment during reactive hyperemia after arterial occlusion and capillary density during venous congestion as the outcome (both in capillaries $/ \mathrm{mm}^{2}$ ) instead of percentage recruitment. Acetylcholine- and local heatinginduced skin vasoreactivity were expressed as percentage increase in perfusion from baseline. In addition, acetylcholine- and local heating-induced skin vasoreactivity and skin flowmotion energy were logarithmically transformed to normalize their skewed distribution. Linear regression analysis was used to evaluate the association between cfPWV and measures of skin microvascular function. Adjustments were made for the 
following potential confounders: age and sex (model 1); additionally for mean arterial pressure and heart rate (model 2); and CVD risk factors: waist-to-hip ratio, fasting plasma glucose, total / high density lipoprotein cholesterol ratio, triglycerides, smoking habits (current, ever and never smoker), use of lipid-lowering medication and (for The Maastricht Study) prior CVD and use of anti-hypertensive medication (model 3). Analyses with flowmotion as the outcome were additionally adjusted for skin temperature in all models. We used interaction terms to explore whether any association differed according to DM2, fasting glucose, age, presence of hypertension, use of anti-hypertensive medication and/or study population (The SUVIMAX2 Study vs. The Maastricht Study). ${ }^{1}$ In addition, analyses were repeated with tertiles of cfPWV. Finally, analyses were repeated with brachial pulse pressure (systolic minus diastolic pressure), a surrogate measure of arterial stiffness, as the determinant instead of cfPWV.

\section{Results}

\section{Analytic sample}

Of the 291 participants of The SUVIMAX2 Study, data were missing on $\operatorname{cfPWV}(n=2)$, capillaroscopy $(n=2)$, skin vasoreactivity $(n=3)$ or potential confounders $(n=3)$. Therefore, 284 participants were eligible for analysis with capillary density and 283 for analysis with skin vasoreactivity, respectively. Of the 866 participants of The Maastricht Study, four participants with type 1 diabetes mellitus were excluded. In the remaining 862 participants, data were missing on $\operatorname{cfPWV}(n=46$; due to logistical reasons $(n=33)$ or insufficient quality $(n=13))$, capillaroscopy $(n=38$; due to logistical reasons $(n=31)$ or insufficient quality $(n=7)$ ), skin flowmotion $(n=128$; due to logistical reasons $(n=97)$ or insufficient quality $(n=31)$ ) or potential confounders $(n=46)$. Therefore, 732 participants were eligible for analysis with capillary density (524 without DM2 and 208 with DM2) and 648 for analysis with skin flowmotion (473 without DM2 and 175 with DM2), respectively.

\section{Clinical characteristics}

Table 9.1 shows the clinical characteristics of The SUVIMAX2 Study and Table 9.2 of The Maastricht Study. Individuals of The SUVIMAX2 Study were, as compared to those of The Maastricht Study without DM2, older and had a better CVD risk factor pattern (Tables 9.1 and 9.2). In addition, in The SUVIMAX2 Study, cfPWV and baseline capillary density were higher than in The Maastricht Study, but capillary recruitment during venous congestion was lower (Tables 9.1 and 9.2). These differences were most likely due to use of different measurement techniques (see below). Within The Maastricht Study, individuals with DM2 were, as compared to those without DM2, older, less often female and had a worse CVD risk factor pattern; and had a higher cfPWV and lower capillary recruitment during reactive hyperemia after arterial occlusion and during venous congestion (Table 9.2). The 
median duration of DM2 was 7 years (interquartile range 3 to 11). Individuals with DM2 were treated with diet only (22\%), oral glucose-lowering medication only (59\%) or insulin (with or without oral glucose-lowering medication; 19\%).

Table 9.1. Study population characteristics according to median cfPWV values for The SUVIMAX2 Study

\begin{tabular}{|c|c|c|c|}
\hline & \multicolumn{3}{|c|}{ The SUVIMAX2 Study } \\
\hline & \multirow[t]{2}{*}{ Total $(n=284)$} & \multicolumn{2}{|c|}{ According to median cfPWV value $(10.5 \mathrm{~m} / \mathrm{s})$} \\
\hline & & Low cfPWV $(n=137)$ & High cfPWV $(n=147)$ \\
\hline \multicolumn{4}{|l|}{ Clinical characteristics } \\
\hline Age, years & $62.2 \pm 5.9$ & $61.5 \pm 5.6$ & $62.9 \pm 6.0$ \\
\hline Women, \% (n) & $48.6(138)$ & $58.4(80)$ & $39.5(58)$ \\
\hline \multicolumn{4}{|l|}{ Smoking status, \% (n) } \\
\hline Never & $50.4(143)$ & $52.6(72)$ & $48.3(71)$ \\
\hline Former & $40.8(116)$ & $35.0(48)$ & $46.3(68)$ \\
\hline Current & $8.8(25)$ & $12.4(17)$ & $5.4(8)$ \\
\hline Prior cardiovascular disease, \% (n) & $0(0)^{A}$ & $0(0)^{A}$ & $0(0)^{A}$ \\
\hline Body mass index, $\mathrm{kg} / \mathrm{m}^{2}$ & $25.1 \pm 3.4$ & $24.9 \pm 3.4$ & $25.3 \pm 3.4$ \\
\hline Systolic blood pressure, mmHg & $119 \pm 13$ & $114 \pm 11$ & $123 \pm 12$ \\
\hline Diastolic blood pressure, $\mathrm{mmHg}$ & $77 \pm 9$ & $74 \pm 8$ & $79 \pm 9$ \\
\hline Hypertension, \% (n) & $0(0)^{A}$ & $0(0)^{A}$ & $0(0)^{A}$ \\
\hline Fasting glucose, mmol/l & $5.4 \pm 0.6$ & $5.3 \pm 0.6$ & $5.4 \pm 0.5$ \\
\hline Total cholesterol, mmol/l & $6.0 \pm 1.0$ & $5.9 \pm 1.0$ & $6.0 \pm 1.0$ \\
\hline Lipid-lowering medication, \% (n) & $8.5(24)$ & $6.6(9)$ & $10.2(15)$ \\
\hline Anti-hypertensive medication, \% (n) & $0(0)^{A}$ & $0(0)^{A}$ & $0(0)^{A}$ \\
\hline \multicolumn{4}{|l|}{ Vascular measures } \\
\hline $\mathrm{cfPWV}, \mathrm{m} / \mathrm{s}$ & $10.9 \pm 2.1$ & $9.4 \pm 0.8$ & $12.4 \pm 1.9$ \\
\hline \multicolumn{4}{|l|}{ Capillary density, capillaries/mm² } \\
\hline Baseline & $90 \pm 16$ & $90 \pm 14$ & $90 \pm 17$ \\
\hline Venous congestion & $96 \pm 17$ & $96 \pm 15$ & $96 \pm 18$ \\
\hline \multicolumn{4}{|l|}{ Capillary recruitment, \% (n) } \\
\hline Venous congestion & $7 \pm 11$ & $7 \pm 11$ & $7 \pm 11$ \\
\hline \multicolumn{4}{|l|}{ Skin vasoreactivity, \% } \\
\hline Acetylcholine-induced & $349(172-572)$ & $364(181-573)$ & $338(159-576)$ \\
\hline Local heating-induced & $568(379-875)$ & $595(393-914)$ & $538(362-856)$ \\
\hline Total skin flowmotion energy, AU & $\mathrm{n} / \mathrm{a}$ & $\mathrm{n} / \mathrm{a}$ & $\mathrm{n} / \mathrm{a}$ \\
\hline \multicolumn{4}{|c|}{$\begin{array}{l}\text { Data are presented as percentage (number) of participants, mean } \pm \text { standard deviation or median (interquartil } \\
\text { range). }\end{array}$} \\
\hline
\end{tabular}


Table 9.2. Study population characteristics according to median cfPWV values for The Maastricht Study

\begin{tabular}{|c|c|c|c|c|c|c|}
\hline & \multicolumn{6}{|c|}{ The Maastricht Study } \\
\hline & \multicolumn{3}{|c|}{ Without type 2 diabetes } & \multicolumn{3}{|c|}{ With type 2 diabetes } \\
\hline & \multirow[t]{2}{*}{$\begin{array}{c}\text { Total } \\
(n=524)\end{array}$} & \multicolumn{2}{|c|}{$\begin{array}{l}\text { According to median } \\
\text { cfPWV value }(8.2 \mathrm{~m} / \mathrm{s})\end{array}$} & \multirow[t]{2}{*}{$\begin{array}{c}\text { Total } \\
(n=208)\end{array}$} & \multicolumn{2}{|c|}{$\begin{array}{l}\text { According to median } \\
\text { cfPWV value }(9.5 \mathrm{~m} / \mathrm{s})\end{array}$} \\
\hline & & $\begin{array}{c}\text { Low cfPWV } \\
(n=260)\end{array}$ & $\begin{array}{c}\text { High } \\
\text { cfPWV } \\
(n=264) \\
\end{array}$ & & $\begin{array}{c}\text { Low } \\
\text { cfPWV } \\
(n=103)\end{array}$ & $\begin{array}{c}\text { High } \\
\text { cfPWV } \\
(n=105)\end{array}$ \\
\hline \multicolumn{7}{|l|}{ Clinical characteristics } \\
\hline Age, years & $58.1 \pm 8.5$ & $54.4 \pm 8.1$ & $61.8 \pm 7.2$ & $63.3 \pm 7.2$ & $61.1 \pm 7.7$ & $65.5 \pm 6.0$ \\
\hline Women, \% (n) & $51.5(270)$ & $58.1(151)$ & 45.1 (119) & $29.3(61)$ & $30.1(31)$ & $28.6(30)$ \\
\hline \multicolumn{7}{|l|}{ Smoking status, \% (n) } \\
\hline Never & $34.3(180)$ & $36.5(95)$ & $32.2(85)$ & $23.1(48)$ & $24.3(25)$ & $21.9(23)$ \\
\hline Former & $50.0(262)$ & $47.7(124)$ & $52.3(138)$ & $62.5(130)$ & $621.1(64)$ & $62.9(66)$ \\
\hline Current & $15.8(83)$ & $16.9(44)$ & $14.8(39)$ & $14.9(31)$ & $14.6(15)$ & $15.2(16)$ \\
\hline Prior cardiovascular disease, $\%$ (n) & $12.8(67)$ & $9.6(25)$ & $15.9(42)$ & $29.3(61)$ & $28.2(29)$ & $30.5(32)$ \\
\hline Body mass index, $\mathrm{kg} / \mathrm{m}^{2}$ & $26.2 \pm 3.9$ & $25.6 \pm 3.6$ & $26.8 \pm 4.1$ & $29.7 \pm 4.7$ & $30.0 \pm 5.1$ & $29.5 \pm 4.3$ \\
\hline Systolic blood pressure, $\mathrm{mmHg}$ & $133 \pm 18$ & $127 \pm 15$ & $140 \pm 17$ & $148 \pm 19$ & $142 \pm 18$ & $154 \pm 18$ \\
\hline Diastolic blood pressure, $\mathrm{mmHg}$ & $76 \pm 11$ & $74 \pm 10$ & $78 \pm 71$ & $79 \pm 10$ & $79 \pm 12$ & $79 \pm 9$ \\
\hline Hypertension, \% (n) & $46.0(241)$ & $29.2(76)$ & $62.5(165)$ & $86.5(180)$ & $81.6(84)$ & $91.4(96)$ \\
\hline Fasting glucose, mmol/l & $5.4 \pm 0.6$ & $5.3 \pm 0.5$ & $5.5 \pm 0.6$ & $7.9 \pm 1.8$ & $7.8 \pm 1.6$ & $8.0 \pm 2.1$ \\
\hline Total cholesterol, mmol/l & $5.5 \pm 1.1$ & $5.5 \pm 1.1$ & $5.6 \pm 1.1$ & $4.5 \pm 1.1$ & $4.4 \pm 0.9$ & $4.6 \pm 2.1$ \\
\hline Lipid-lowering medication, \% (n) & $19.8(104)$ & $15.0(39)$ & $24.6(65)$ & $75.5(157)$ & $77.7(80)$ & $73.3(77)$ \\
\hline Anti-hypertensive medication, $\%$ ( $n$ ) & $26.7(140)$ & $17.7(46)$ & $35.6(94)$ & $70.7(147)$ & $70.9(73)$ & $70.5(74)$ \\
\hline RAAS inhibitors & $18.9(99)$ & $11.5(30)$ & $26.1(69)$ & $57.2(119)$ & $57.3(59)$ & $57.1(60)$ \\
\hline Diuretics & $10.5(55)$ & $6.9(18)$ & $14.0(37)$ & $26.0(54)$ & $25.2(26)$ & $26.7(28)$ \\
\hline Calcium channel blockers & $4.8(25)$ & $2.7(7)$ & $6.8(18)$ & $17.3(36)$ & $12.6(13)$ & $21.9(23)$ \\
\hline Beta-blockers & $11.5(60)$ & $9.2(24)$ & $13.6(36)$ & $35.1(73)$ & $32.0(33)$ & $38.1(40)$ \\
\hline \multicolumn{7}{|l|}{ Vascular measures } \\
\hline $\mathrm{cfPWV}, \mathrm{m} / \mathrm{s}$ & $8.5 \pm 1.8$ & $7.2 \pm 0.7$ & $9.9 \pm 1.5$ & $9.9 \pm 2.4$ & $8.0 \pm 1.0$ & $11.7 \pm 2.0$ \\
\hline \multicolumn{7}{|l|}{ Capillary density, capillaries $/ \mathrm{mm}^{2}$} \\
\hline Baseline & $72 \pm 17$ & $72 \pm 17$ & $72 \pm 18$ & $77 \pm 18$ & $76 \pm 18$ & $78 \pm 18$ \\
\hline Hyperemia & $104 \pm 17$ & $104 \pm 17$ & $103 \pm 18$ & $102 \pm 19$ & $100 \pm 20$ & $103 \pm 17$ \\
\hline Venous congestion & $104 \pm 18$ & $105 \pm 17$ & $104 \pm 18$ & $102 \pm 19$ & $101 \pm 21$ & $103 \pm 17$ \\
\hline \multicolumn{7}{|l|}{ Capillary recruitment, \% (n) } \\
\hline Reactive Hyperemia & $46 \pm 30$ & $49 \pm 30$ & $50 \pm 30$ & $35 \pm 25$ & $34 \pm 25$ & $35 \pm 25$ \\
\hline Venous congestion & $50 \pm 32$ & $50 \pm 33$ & $51 \pm 32$ & $35 \pm 26$ & $35 \pm 26$ & $36 \pm 26$ \\
\hline Total skin flowmotion energy, $\mathrm{AU}$ & $14(9-21)$ & $14(8-20)$ & $15(10-21)$ & $15(10-23)$ & $14(10-21)$ & $18(10-23)$ \\
\hline
\end{tabular}

Data are presented as percentage (number) of participants, mean \pm standard deviation or median (interquartile range).

Abbreviations as in Table 9.1. 


\section{Association between cfPWV and baseline capillary density and capillary recruitment}

In The SUVIMAX2 Study, cfPWV was not associated with baseline capillary density and capillary recruitment during venous congestion, after adjustment for age and sex (Table 9.3, model 1). Further adjustments for mean arterial pressure and heart rate (model 2) and CVD risk factors (model 3 ) did not materially change these results. Similarly, in The Maastricht Study, both in individuals without and with DM2, cfPWV was not associated with baseline capillary density and capillary recruitment during reactive hyperemia after arterial occlusion or during venous congestion (Table 9.3, models 1 to 3 ).

\section{Association between cfPWV and skin vasoreactivity}

In The SUVIMAX2 Study, cfPWV was not associated with acetylcholine- or local heatinginduced skin vasoreactivity (Table 9.4, models 1 to 3 ).

\section{Association between cfPWV and skin flowmotion}

In The Maastricht Study, both in individuals without and with DM2, cfPWV was not associated with total skin flowmotion energy (Table 9.4, models 1 to 3), or with any of the individual skin flowmotion components (data not shown).

Table 9.3. Association between carotid-femoral pulse wave velocity and skin capillary density and recruitment

\begin{tabular}{|c|c|c|c|}
\hline & $\begin{array}{l}\text { Baseline capillary density } \\
\left(\text { capillaries } / \mathrm{mm}^{2} \text { ) }\right.\end{array}$ & $\begin{array}{c}\text { Recruitment during reactive } \\
\text { hyperemia after } \\
\text { arterial occlusion (\%) }\end{array}$ & $\begin{array}{l}\text { Recruitment during venous } \\
\text { congestion (\%) }\end{array}$ \\
\hline \multicolumn{4}{|c|}{ Regression coefficient (95\% confidence interval) for +1 SD cfPWV } \\
\hline \multicolumn{4}{|c|}{ The SUVIMAX2 Study } \\
\hline 1 & $-0.48(-2.37 ; 1.41)$ & $\mathrm{n} / \mathrm{a}$ & $0.54(-0.74 ; 1.81)$ \\
\hline 2 & $-0.56(-2.57 ; 1.44)$ & $\mathrm{n} / \mathrm{a}$ & $0.59(-0.76 ; 1.94)$ \\
\hline 3 & $-0.27(-2.32 ; 1.77)$ & $\mathrm{n} / \mathrm{a}$ & $0.62(-0.77 ; 2.01)$ \\
\hline \multicolumn{4}{|c|}{ The Maastricht Study } \\
\hline \multicolumn{4}{|c|}{ Individuals without type 2 diabetes mellitus } \\
\hline 1 & $-1.63(-3.31 ; 0.06)$ & $1.22(-0.41 ; 2.84)$ & $1.50(-0.25 ; 3.25)$ \\
\hline 2 & $-1.56(-3.51 ; 0.39)$ & $0.78(-1.10 ; 2.66)$ & $1.01(-1.01 ; 3.03)$ \\
\hline 3 & $-1.20(-3.17 ; 0.77)$ & $0.41(-1.49 ; 2.31)$ & $0.62(-1.42 ; 2.67)$ \\
\hline \multicolumn{4}{|c|}{ Individuals with type 2 diabetes mellitus } \\
\hline 1 & $0.79(-1.84 ; 3.42)$ & $0.003(-1.53 ; 1.53)$ & $0.14(-1.45 ; 1.73)$ \\
\hline 2 & $0.06(-2.82 ; 2.94)$ & $0.32(-1.35 ; 1.99)$ & $0.39(-1.34 ; 2.13)$ \\
\hline 3 & $0.12(-2.74 ; 3.00)$ & $0.38(-1.29 ; 2.06)$ & $0.42(-1.33 ; 2.16)$ \\
\hline
\end{tabular}

Model 1: adjusted for age and sex; model 2: additionally adjusted for heart rate and mean arterial pressure; model 3: additionally adjusted for waist-to-hip ratio, smoking habits, fasting glucose, total / high density lipoprotein cholesterol ratio, triglycerides, use of lipid-lowering medication and (for The Maastricht Study) prior cardiovascular disease and use of anti-hypertensive medication.

Abbreviations as in Table 9.1. 
Table 9.4. Association between carotid-femoral pulse wave velocity on the one hand and acetylcholine- and local heating-induced skin vasoreactivity and skin flowmotion on the other

\begin{tabular}{cccc}
\hline & $\begin{array}{c}\text { Acetylcholine-induced } \\
\text { skin vasoreactivity }(\%)^{\mathrm{A}}\end{array}$ & $\begin{array}{c}\text { Local heating-induced } \\
\text { skin vasoreactivity }(\%)^{\mathrm{A}}\end{array}$ & $\begin{array}{c}\text { Total skin flowmotion } \\
\text { energy }(\mathrm{AU})^{\mathrm{A}}\end{array}$ \\
\cline { 2 - 4 } \multicolumn{2}{c}{ Regression coefficient } & $(95 \%$ confidence interval) for +1 SD cfPWV \\
1 & $-0.02(-0.14 ; 0.10)$ & $0.03(-0.07 ; 0.12)$ & $\mathrm{n} / \mathrm{a}$ \\
2 & $-0.07(-0.19 ; 0.06)$ & $-0.01(-0.11 ; 0.09)$ & $\mathrm{n} / \mathrm{a}$ \\
3 & $-0.09(-0.21 ; 0.04)$ & $-0.02(-0.12 ; 0.08)$ & $\mathrm{n} / \mathrm{a}$ \\
\hline
\end{tabular}

The Maastricht Study

Individuals without type 2 diabetes mellitus

\begin{tabular}{|c|c|c|c|}
\hline 1 & $\mathrm{n} / \mathrm{a}$ & $\mathrm{n} / \mathrm{a}$ & $-0.01(-0.07 ; 0.06)$ \\
\hline 2 & $\mathrm{n} / \mathrm{a}$ & $\mathrm{n} / \mathrm{a}$ & $-0.02(-0.10 ; 0.06)$ \\
\hline 3 & $\mathrm{n} / \mathrm{a}$ & $\mathrm{n} / \mathrm{a}$ & $-0.01(-0.09 ; 0.07)$ \\
\hline \multicolumn{4}{|c|}{ ndividuals with type 2 diabetes mellitus } \\
\hline 1 & $\mathrm{n} / \mathrm{a}$ & $\mathrm{n} / \mathrm{a}$ & $-0.05(-0.15 ; 0.04)$ \\
\hline 2 & $\mathrm{n} / \mathrm{a}$ & $\mathrm{n} / \mathrm{a}$ & $-0.05(-0.15 ; 0.06)$ \\
\hline 3 & $\mathrm{n} / \mathrm{a}$ & $\mathrm{n} / \mathrm{a}$ & $-0.04(-0.14 ; 0.07)$ \\
\hline
\end{tabular}

A Data were logarithmically transformed. Model 1: adjusted for age, sex and skin temperature; model 2: additionally adjusted for heart rate and mean arterial pressure; model 3: additionally adjusted for waist-to-hip ratio, smoking habits, fasting glucose, total / high density lipoprotein cholesterol ratio, triglycerides, use of lipidlowering medication and (for The Maastricht Study) prior cardiovascular disease and use of anti-hypertensive medication.

Abbreviations as in Table 9.1.

\section{Additional analyses}

There was no interaction with DM2, fasting glucose, age, presence of hypertension, or use of anti-hypertensive medication for any of the skin microvascular function measures in either The SUVIMAX2 Study or The Maastricht Study ( $P$ for interaction $>.09$, after adjustment for all potential confounders). In addition, there was no interaction with the study populations of The SUVIMAX2 Study vs. The Maastricht Study for baseline capillary density and capillary recruitment during venous congestion ( $P$ for interaction $>.67$, after adjustment for all potential confounders). When we used absolute recruitment during hyperemia after arterial occlusion and capillary density during venous congestion, instead of percentage recruitment, results were qualitatively similar (data not shown). In addition, when the analyses were repeated with tertiles of cfPWV, instead of per higher SD, results were qualitatively similar (data not shown). Results were qualitatively similar when we used brachial pulse pressure as the determinant instead of cfPWV (data not shown). Finally, when results of both studies were pooled, results were qualitatively similar (data not shown). 


\section{Discussion}

The main finding of the present study is that, in middle-aged individuals with or without DM2, arterial stiffness was not associated with skin microvascular dysfunction, as determined by baseline capillary density; capillary recruitment during reactive hyperemia after arterial occlusion and during venous congestion; endothelium-dependent and independent skin vasoreactivity; and skin flowmotion.

Strengths of the present study include its extensive measurement of skin microvascular function and assessment of arterial stiffness by cfPWV, which is considered the "gold standard" measurement. ${ }^{26}$ In addition, the associations were investigated in two large studies performed by independent investigators. It is therefore unlikely that the negative findings are due to technical measurement issues or insufficient statistical power, although we did not do a formal power calculation. Furthermore, The Maastricht Study is a population-based study with oversampling of individuals with DM2. This allowed us to investigate any potential contrast between individuals with and without DM2 in the association between cfPWV and microvascular function.

Limitations of the present study include its cross-sectional design; we therefore cannot exclude the possibility of an association between greater arterial stiffness and decrement of skin microvascular function over time. In addition, not all measures of skin microvascular function were available in each study. Finally, the intra- and inter-observer reproducibility was not tested of the laser Doppler flowmetry measurements in The SUVIMAX2 Study and The Maastricht Study, and of the cfPWV measurement in The SUVIMAX2 Study. However, previous studies ${ }^{29-32}$ have shown that laser Doppler flowmetry ${ }^{29-31}$ and cfPWV measurements ${ }^{33}$ have a relatively high reproducibility.

The lack of an association between arterial stiffness and skin microvascular function suggests that arterial stiffness does not lead to generalized microvascular dysfunction. There is substantial evidence that the skin microcirculation is representative of the microcirculation in general. ${ }^{11,13-15,18}$ Possibly, the microcirculation of most organs is able to protect itself against the detrimental effects of increased arterial stiffness and pressure and flow pulsatility. This may be due to the fact that most organs have relatively high microvascular impedance. Therefore, most of the increased pulsatile energy is dissipated by arteries and large arterioles proximal to the capillaries. ${ }^{1}$ In addition, a vascular remodelling response may be induced by the increased pulsatile load, which raises vascular resistance and, thereby, limits penetration of the pulsatile load into its microvascular bed. Other data ${ }^{6,34,35}$ suggest that a remodelling response does occur in relatively large arterioles. For example, previous studies ${ }^{35-37}$ have shown an association between greater arterial stiffness and a higher wall-to-lumen ratio of large arterioles in 
the subcutis ${ }^{35,36}$ and retina. ${ }^{37}$ The present study suggests that such remodelling, however, does not occur in small arterioles, because arterial stiffness was not associated with impaired acetylcholine-induced vasoreactivity. Remodelling of small arterioles is usually accompanied by impairment of this response. ${ }^{38}$ In addition, the present study suggests that the previously observed remodelling response in large arterioles is effective, because arterial stiffness was not associated with capillary rarefaction. This indicates that capillaries are "protected" against the potential detrimental effects of increased pressure and flow pulsatility.

The present results do, however, not exclude the possibility that arterial stiffness is associated with microvascular dysfunction in the brain, eye and kidney. The microvascular beds of these organs are characterized by low impedance, and, therefore, these organs are especially vulnerable for the detrimental effects of an increased pulsatile load. ${ }^{1,2}$ Indeed, previous studies have shown an association between arterial stiffness and markers of microvascular dysfunction in these organs. ${ }^{3-5}$

In The SUVIMAX2 Study and The Maastricht Study, slightly different measurement techniques were used to assess cfPWV and capillary density, which can explain the difference in absolute values of these measures between the studies. Median cfPWV was higher in The SUVIMAX2 Study than in The Maastricht Study, most likely due to a difference in the calculation of pulse wave travel distance. In The SUVIMAX2 Study, the total carotid to femoral distance was used as travel distance, whereas in The Maastricht Study $80 \%$ of the total carotid to femoral distance was used. In addition, in The SUVIMAX2 Study, baseline capillary density was higher and capillary recruitment during venous congestion was lower than in The Maastricht Study. This may be due to use of a higher microscopic magnification in The SUVIMAX2 Study (200x vs. 100x), differences in the method used to count capillaries (using primarily freeze-framed reproductions in The SUVIMAX2 Study vs. running movie files in The Maastricht Study) and lower cuff inflation during venous congestion $(50 \mathrm{mmHg}$ in The SUVIMAX2 Study vs. $60 \mathrm{mmHg}$ in The Maastricht Study). In addition, the differences in absolute values of the vascular measures may be due to the differences in clinical characteristics between The SUVIMAX2 Study and The Maastricht Study. The net effect of such differences in characteristics is, however, difficult to quantify, because the different characteristics (both measured and unmeasured) may have influenced absolute values in various manners. Furthermore, it is difficult to disentangle the effects of the differences in clinical characteristics from the effects of the differences in measurement techniques between the two studies.

In conclusion, the present study shows that greater arterial stiffness is not associated with skin microvascular dysfunction in middle-aged individuals either with or without DM2. 


\section{References}

1. Mitchell GF. Effects of central arterial aging on the structure and function of the peripheral vasculature: implications for end-organ damage. J Appl Physiol (1985). 2008;105:1652-1660.

2. O'Rourke MF, Safar ME. Relationship between aortic stiffening and microvascular disease in brain and kidney: cause and logic of therapy. Hypertension. 2005;46:200-204.

3. Mitchell GF, van Buchem MA, Sigurdsson S, et al. Arterial stiffness, pressure and flow pulsatility and brain structure and function: the Age, Gene/Environment Susceptibility--Reykjavik study. Brain. 2011;134:3398-3407.

4. Cheung $\mathrm{N}$, Sharrett AR, Klein R, et al. Aortic distensibility and retinal arteriolar narrowing: the multiethnic study of atherosclerosis. Hypertension. 2007;50:617-622.

5. Briet M, Boutouyrie P, Laurent S, London GM. Arterial stiffness and pulse pressure in CKD and ESRD. Kidney Int. 2012;82:388-400.

6. Mitchell GF, Vita JA, Larson MG, et al. Cross-sectional relations of peripheral microvascular function, cardiovascular disease risk factors, and aortic stiffness: the Framingham Heart Study. Circulation. 2005;112:3722-3728.

7. Kim ES, Moon SD, Kim HS, et al. Diabetic peripheral neuropathy is associated with increased arterial stiffness without changes in carotid intima-media thickness in type 2 diabetes. Diabetes Care. 2011;34:1403-1405.

8. Yasuno S, Ueshima K, Oba K, et al. Is pulse pressure a predictor of new-onset diabetes in high-risk hypertensive patients?: a subanalysis of the Candesartan Antihypertensive Survival Evaluation in Japan (CASE-J) trial. Diabetes Care. 2010;33:1122-1127.

9. Hirose K, Tomiyama H, Okazaki R, et al. Increased pulse wave velocity associated with reduced calcaneal quantitative osteo-sono index: possible relationship between atherosclerosis and osteopenia. J Clin Endocrinol Metab. 2003;88:2573-2578.

10. Gronenschild EH, Muris DM, Schram MT, Karaca U, Stehouwer CD, Houben AJ. Semi-automatic assessment of skin capillary density: proof of principle and validation. Microvascular Res. 2013;90:192-198.

11. Holowatz LA, Thompson-Torgerson CS, Kenney WL. The human cutaneous circulation as a model of generalized microvascular function. J Appl Physiol (1985). 2008;105:370-372.

12. Czernichow S, Greenfield JR, Galan P, et al. Macrovascular and microvascular dysfunction in the metabolic syndrome. Hypertens Res. 2010;33:293-297.

13. IJzerman RG, de Jongh RT, Beijk MA, et al. Individuals at increased coronary heart disease risk are characterized by an impaired microvascular function in skin. Eur J Clin Invest. 2003;33:536-542.

14. Czernichow S, Greenfield JR, Galan P, et al. Microvascular dysfunction in healthy insulin-sensitive overweight individuals. J Hypertens. 2010;28:325-332.

15. de Jongh RT, Serne EH, RG IJ, de Vries G, Stehouwer CD. Impaired microvascular function in obesity: implications for obesity-associated microangiopathy, hypertension, and insulin resistance. Circulation. 2004;109:2529-2535.

16. Jonk AM, Houben AJ, Schaper NC, et al. Meal-related increases in microvascular vasomotion are impaired in obese individuals: a potential mechanism in the pathogenesis of obesity-related insulin resistance. Diabetes Care. 2011;34 Suppl 2:S342-348.

17. Serne EH, Gans RO, ter Maaten JC, Tangelder GJ, Donker AJ, Stehouwer CD. Impaired skin capillary recruitment in essential hypertension is caused by both functional and structural capillary rarefaction. Hypertension. 2001;38:238-242.

18. Meijer RI, De Boer MP, Groen MR, et al. Insulin-induced microvascular recruitment in skin and muscle are related and both are associated with whole-body glucose uptake. Microcirculation. 2012;19:494500 . 
19. Rossi M, Taddei S, Fabbri A, et al. Cutaneous vasodilation to acetylcholine in patients with essential hypertension. J Cardiovasc Pharmacol. 1997;29:406-411.

20. Debbabi H, Uzan L, Mourad JJ, Safar M, Levy BI, Tibirica E. Increased skin capillary density in treated essential hypertensive patients. Am J Hypertens. 2006;19:477-483.

21. Sandeman DD, Shore AC, Tooke JE. Relation of skin capillary pressure in patients with insulindependent diabetes mellitus to complications and metabolic control. N Eng J Med. 1992;327:760-764.

22. Hercberg S, Galan P, Preziosi P, et al. The SU.VI.MAX Study: a randomized, placebo-controlled trial of the health effects of antioxidant vitamins and minerals. Arch Intern Med. 2004;164:2335-2342.

23. Schram MT, Sep SJ, Kallen van der CJ, Dagnelie PC, Koster A, Schaper N, Henry RM, Stehouwer CD. The Maastricht Study: An Extensive Phenotyping Study on Determinants of Type 2 Diabetes, its Complications and its Comorbidities. Eur J Epid 2014;29:439-451.

24. Temmar M, Liabeuf S, Renard C, et al. Pulse wave velocity and vascular calcification at different stages of chronic kidney disease. J Hypertens. 2010;28:163-169.

25. Karatzi K, Protogerou A, Kesse-Guyot E, et al. Associations between dietary patterns and skin microcirculation in healthy subjects. Arterioscler Thromb Vasc Biol. 2014;34:463-469.

26. Van Bortel LM, Laurent S, Boutouyrie $P$, et al. Expert consensus document on the measurement of aortic stiffness in daily practice using carotid-femoral pulse wave velocity. J Hypertens. 2012;30:445448 .

27. Schmidt-Lucke C, Borgstrom P, Schmidt-Lucke JA. Low frequency flowmotion/(vasomotion) during patho-physiological conditions. Life Sci. 2002;71:2713-2728.

28. Stefanovska A, Bracic M, Kvernmo HD. Wavelet analysis of oscillations in the peripheral blood circulation measured by laser Doppler technique. IEEE Trans Biomed Eng. 1999;46:1230-1239.

29. Boignard A, Salvat-Melis M, Carpentier PH, et al. Local hyperemia to heating is impaired in secondary Raynaud's phenomenon. Arthritis Res Ther. 2005;7:R1103-1112.

30. Cracowski JL, Minson CT, Salvat-Melis M, Halliwill JR. Methodological issues in the assessment of skin microvascular endothelial function in humans. Trends Pharmacol Sci. 2006;27:503-508.

31. Kubli S, Waeber B, Dalle-Ave A, Feihl F. Reproducibility of laser Doppler imaging of skin blood flow as a tool to assess endothelial function. J Cardiovasc Pharmacol. 2000;36:640-648.

32. Laurent S, Cockcroft J, Van Bortel L, et al. Expert consensus document on arterial stiffness: methodological issues and clinical applications. Eur Heart J. 2006;27:2588-2605.

33. Laurent S, Briet M, Boutouyrie P. Large and small artery cross-talk and recent morbidity-mortality trials in hypertension. Hypertension. 2009;54:388-392.

34. James MA, Watt PA, Potter JF, Thurston H, Swales JD. Pulse pressure and resistance artery structure in the elderly. Hypertension. 1995;26:301-306.

35. Muiesan ML, Salvetti M, Rizzoni D, et al. Pulsatile hemodynamics and microcirculation: evidence for a close relationship in hypertensive patients. Hypertension. 2013;61:130-136.

36. Salvetti M, Agabiti Rosei C, Paini A, et al. Relationship of wall-to-lumen ratio of retinal arterioles with clinic and 24-hour blood pressure. Hypertension. 2014;63:1110-1115.

37. Ott C, Raff U, Harazny JM, Michelson G, Schmieder RE. Central pulse pressure is an independent determinant of vascular remodeling in the retinal circulation. Hypertension. 2013;61:1340-1345.

38. Turner J, Belch JJ, Khan F. Current concepts in assessment of microvascular endothelial function using laser Doppler imaging and iontophoresis. Trends Cardiovasc Med. 2008;18:109-116. 
Arterial stiffness and skin microvascular function | 215 


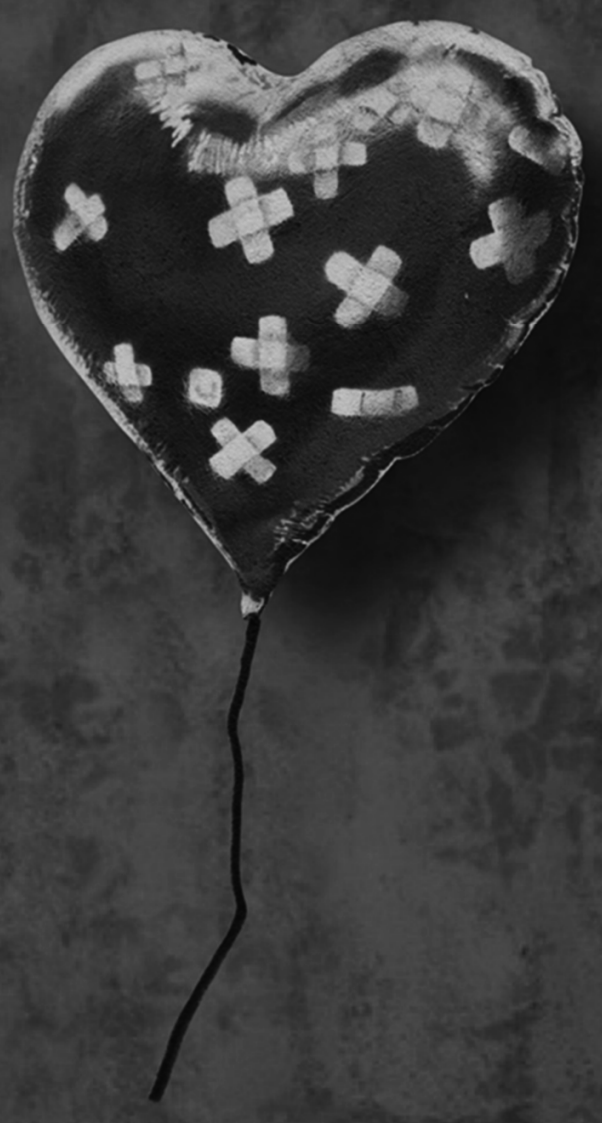

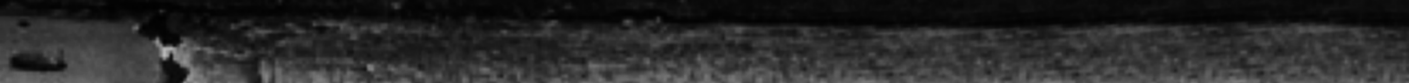
1. 1. 1. 


\section{Chapter 10}

General discussion 

Life expectancy in the Netherlands and much of the rest of the world has dramatically increased and will continue to do so in the next decades. ${ }^{1}$ Ageing is associated with a greatly increased risk of vascular-related diseases of the heart and brain, including coronary heart disease, heart failure and stroke (cardiovascular diseases) and (vascular) dementia and depression. In recent years, emerging evidence indicates that dysfunction of various elements of the vascular system plays an important role in the pathogenesis of these diseases. ${ }^{2-4}$ Vascular dysfunction includes dysfunction of large arteries (due to arterial stiffness), the microcirculation (microvascular dysfunction) and endothelium (endothelial dysfunction). Indeed, recent statements of the European Society of Hypertension/European Society of Cardiology ${ }^{5}$ and the American Heart Association/American Stroke Association ${ }^{6}$ have indicated arterial stiffness and endothelial dysfunction as important, potentially modifiable risk factors for cardiovascular disease and cognitive impairment. However, the exact role of arterial stiffness and microvascular and endothelial dysfunction in the pathogenesis of these diseases is incompletely understood and their clinical utility remains controversial. ${ }^{7,8}$ The present thesis therefore sought to further investigate, in a series of epidemiological studies, the role of arterial stiffness and microvascular and endothelial dysfunction in the pathogenesis of cardiovascular disease, cognitive impairment and depressive symptoms. This chapter discusses, in light of current knowledge, the key findings of the present thesis and their potential clinical implications. In addition, methodological considerations and directions for future research will be addressed.

\section{Key findings and clinical implications}

\section{Endothelial dysfunction and type $\mathbf{2}$ diabetes synergistically increase cardiovascular risk}

In the pathogenesis of cardiovascular events, true interaction (synergy) between risk factors appears rare, i.e. most studies ${ }^{9-12}$ find that risk factors act, and thus increase cardiovascular risk, independently of each other. From a clinical point of view, detection of interaction is, however, important as this identifies key therapeutic targets: interventions aimed at such risk factors are potentially more efficacious than treatment of risk factors which do not interact. In chapter two of this thesis, we used prospective data of The Hoorn Study to evaluate the interaction between endothelial dysfunction on the one hand and type 2 diabetes, impaired glucose metabolism and insulin resistance on the other with regard to the risk of cardiovascular events. We investigated this interaction as it has been suggested that individuals with type 2 diabetes are extremely sensitive to the adverse cardiovascular effects of endothelial dysfunction. ${ }^{13,14}$ If true, this implies that endothelial dysfunction and type 2 diabetes synergistically increase cardiovascular risk. This may be due to a reciprocal association between endothelial dysfunction and type 2 diabetes, in which endothelial dysfunction acts as both cause ${ }^{15,16}$ and consequence ${ }^{17}$ of type 2 
diabetes. In addition, type 2 diabetes may amplify the detrimental effects of endothelial dysfunction on atherothrombosis. ${ }^{18}$ In accordance with this hypothesis, two recent studies $^{13,14}$ showed interaction, on a multiplicative scale, with regard to cardiovascular event risk, between type 2 diabetes and endothelial dysfunction. Both studies defined endothelial dysfunction by increased levels of endothelium-derived circulating biomarkers. In chapter two of this thesis, it is shown that such interaction is also present between type 2 diabetes and impaired endothelium-dependent flow-mediated dilatation, a key functional estimate of endothelium-dependent, nitric oxide-mediated dilatation. ${ }^{7}$ In addition, interaction on an additive scale (potentially causal interaction ${ }^{19}$ ) was demonstrated, and such interaction was already present in individuals with impaired glucose metabolism or insulin resistance. This chapter therefore provides strong evidence in favour of causal interaction between endothelial dysfunction and type 2 diabetes in the pathogenesis of cardiovascular events. This interaction is important as it suggests that endothelial dysfunction may act at least partially as the underlying phenomenon which explains the two to three times higher cardiovascular risk seen in type 2 diabetes. ${ }^{18}$ This identifies endothelial dysfunction as a key therapeutic target for lowering of cardiovascular risk in type 2 diabetes. In addition, the fact that an interaction was already present in individuals with impaired glucose metabolism and insulin resistance suggests that endothelial dysfunction is an early therapeutic target even before onset of type 2 diabetes.

\section{Stiffening of elastic and muscular segments: distinct pathways in the pathogenesis of cardiovascular events}

There are substantial differences in properties between elastic and muscular segments, and it has been suggested that stiffening of these segments are differentially associated with cardiovascular events. ${ }^{20,21}$ Stiffness of elastic segments (e.g. the carotid artery and ascending aorta) may be more strongly associated with stroke than coronary heart disease, because stiffening of these segments leads to a high pulsatile pressure and flow load on the brain. ${ }^{22}$ In addition, stiffening of the carotid artery may lead to stroke through local development of (rupture-prone) atherosclerotic plaques. ${ }^{23,24}$ In contrast, stiffness of muscular segments (e.g. the femoral artery and descending aorta) may be more strongly associated with coronary heart disease events than stroke, because muscular and coronary arteries show similar arterial wall characteristics (i.e. presence of abundant smooth muscle cells ${ }^{21}$ and a high collagen/elastin ratio ${ }^{25}$ ), and, therefore, stiffening of muscular segments may serve as a proxy for stiffening of the coronary vasculature. In chapter three of the present thesis, we used local distensibility measurements of the carotid and femoral arteries to investigate elastic and muscular artery stiffness. In line with the above hypothesis, the findings indicate that greater carotid and femoral stiffness are associated with a higher cardiovascular event incidence and greater all-cause mortality risk, independently of each other, and independently of carotid-femoral pulse wave 
velocity. In chapter four, we further elaborated upon these findings and performed a systematic review and an aggregate data and an individual participant data meta-analysis on the association between carotid stiffness and incident cardiovascular events. The results showed that greater carotid stiffness is associated with a higher stroke incidence, but not with coronary heart disease events. The association between carotid stiffness and incident stroke was independent of cardiovascular risk factors, and independent of carotid-femoral pulse wave velocity. In addition, estimation of carotid stiffness modestly improved stroke risk prediction beyond Framingham stroke risk score factors and carotidfemoral pulse wave velocity. From a clinical point of view, these observations are important, as they identify carotid and femoral stiffness as potential separate targets for stroke and coronary heart disease risk lowering therapy. In addition, the findings provide proof of principle that carotid stiffness can have additional value as a risk predictor of stroke beyond the Framingham stroke risk score factors and carotid-femoral pulse wave velocity.

Arterial stiffness and endothelial dysfunction: causes of cerebral microvascular damage, cognitive impairment and depressive symptoms

Increased arterial stiffness leads to an increased pulsatile pressure and flow load, which can damage the microcirculation. ${ }^{26-28}$ The brain may be particularly prone to the detrimental effects of this increased load, because its microcirculation is characterized by low impedance, allowing the pulsatile load to penetrate deeply into its microvascular bed. ${ }^{27,28}$ This increased pulsatile load may directly cause cerebral microvascular dysfunction and damage, despite blood pressure-related protective auto-regulatory mechanisms. Alternatively, the increased pulsatile load may induce a microvascular remodelling response, which initially serves to limit the penetration of the pulsatile load in the microcirculatory system by raising vascular resistance. Yet, this protective response may ultimately become unfavourable leading to impaired vasoreactivity and microvascular ischemia. ${ }^{27,28}$ It is, moreover, likely that these mechanisms operate simultaneously (see Figure 10.1). In addition, increased arterial stiffness may cause excessive blood pressure variability, ${ }^{29,30}$ which may further sensitize high-flow organs to the harmful effects of impaired microvascular vasoreactivity. ${ }^{28}$ Furthermore, a recent study ${ }^{31}$ has suggested that greater arterial stiffness may lead to cerebral (microvascular) damage via acceleration of cerebral $\beta$-amyloid accumulation, although the exact mechanism underlying this association is incompletely understood. Finally, endothelial dysfunction may lead to cerebral microvascular damage via multiple mechanisms, including impairment of the process of neurogenesis, ${ }^{32}$ impaired cerebral blood flow regulation ${ }^{33}$ and disturbance of blood-brain barrier function. ${ }^{34,35}$ In fact, endothelial and microvascular dysfunction are closely related, because most (98\%) of the endothelium is located in the microcirculation. $^{36}$ 


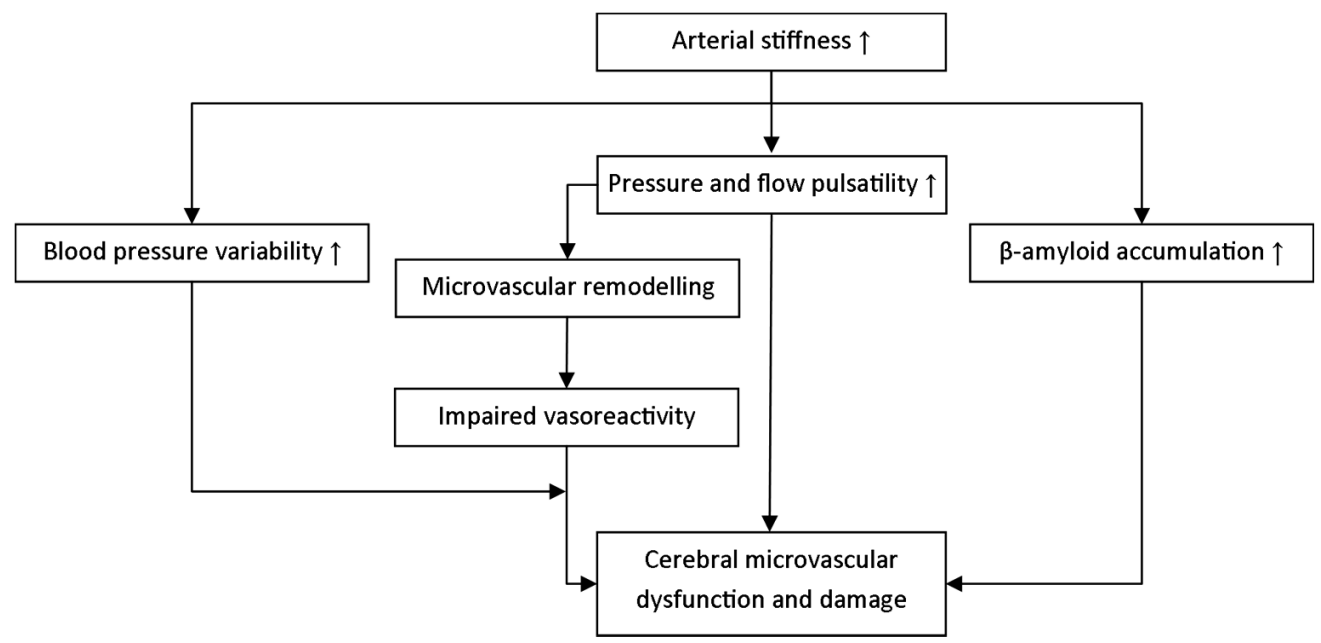

Figure 10.1. Potential pathways through which arterial stiffness can lead to cerebral microvascular dysfunction and damage.

Cerebral microvascular damage, in turn, leads to neuronal cell death, diminished neuronal connectivity and, ultimately, dysfunction of the brain. ${ }^{35,37}$ Brain dysfunction can manifest itself as cognitive impairment, including dementia. In addition, it has been suggested ${ }^{38,39}$ that cerebral microvascular damage leads to depression via disruption of deep and frontal brain structures or their connecting pathways involved in mood regulation, in particular in older individuals (vascular depression hypothesis). Depression has a bimodal age distribution, with peaks in the early third and in ninth decades, suggesting the presence of different causes of depression in young and older individuals. ${ }^{40}$ In line with the vascular depression hypothesis, a recent randomized clinical trial ${ }^{41}$ showed that nimodipine, a drug with vasoprotective properties, reduced time to remission of late-life depression.

Chapters five to eight of this thesis provide further evidence of the existence of an association between vascular dysfunction on the one hand and cerebral microvascular damage and brain dysfunction on the other. The systematic review and aggregate data meta-analysis in chapter five show that a consistent association exists between greater arterial stiffness on the one hand and cerebral small vessel disease and cognitive impairment on the other. In addition, the results in chapters six to eight demonstrate that: 1) various magnetic resonance imaging (MRI) markers of cerebral small vessel disease are strongly associated with a higher depressive symptom incidence (chapter six); 2) cerebral small vessel disease located in the deep (sub-cortical) brain region (i.e. internal and external capsules, thalamus, hippocampus and amygdala combined) is, as compared to disease in other brain regions, more strongly associated with a higher depressive symptom incidence (chapter six); 3) greater arterial stiffness is associated with more (severe) depressive symptoms, and this association is in part mediated by cerebral small vessel disease (chapter seven); and 4) endothelial dysfunction is associated with more 
(severe) depressive symptoms (chapter eight). Taken together, these chapters suggest that both arterial stiffness and endothelial dysfunction can lead to microvascular damagerelated brain dysfunction, which can manifest itself as cognitive impairment and/or depressive symptoms. From a clinical point of view, these associations are important as they suggest that efforts at favourably influencing arterial stiffness and endothelial dysfunction can have significant public health implications via prevention of dementia and depression.

\section{Arterial stiffness does not lead to generalized microvascular dysfunction}

It has been hypothesized ${ }^{42}$ that arterial stiffness can also lead to microvascular dysfunction and damage in other organs than the brain (generalized microvascular dysfunction), and that this may explain the association between arterial stiffness and different diseases, including peripheral neuropathy, ${ }^{43}$ type 2 diabetes ${ }^{44}$ and osteoporosis. ${ }^{45}$ However, evidence of an association between arterial stiffness and markers of generalized microvascular dysfunction is lacking. The skin microcirculation is a representative vascular bed to examine generalized microvascular phenomena. ${ }^{46-48}$ In chapter nine, we therefore investigated the association between arterial stiffness and skin microvascular function. As it has been hypothesized that individuals with type 2 diabetes are particularly prone to the detrimental effects of increased pressure and flow pulsatility on the microcirculation, because type 2 diabetes is associated with increased microvascular perfusion, ${ }^{28,49,50}$ we additionally investigated whether any association between stiffness and microvascular function is stronger in individuals with type 2 diabetes as compared to those without type 2 diabetes. However, in contrast to the above hypothesis, the results of this chapter show that arterial stiffness is not associated with skin microvascular function, irrespective of the presence of type 2 diabetes. This suggests that arterial stiffness alone does not lead to generalized microvascular dysfunction. Possibly, the microcirculation of most organs is able to protect itself against the detrimental effects of increased arterial stiffness and pressure and flow pulsatility. This may be due to the fact that most organs, with the exception of the brain and kidney, have relatively high microvascular impedance. ${ }^{28}$ Therefore, most of the increased pulsatile energy is dissipated by arteries and large arterioles proximal to the capillaries.

\section{Pathophysiological model}

Based on findings of the present thesis, we propose the following pathophysiological model for the interrelated role of arterial stiffness and microvascular and endothelial dysfunction in the pathogenesis cardiovascular disease, cognitive impairment and depressive symptoms (see Figure 10.2). Endothelial dysfunction is associated with a marked increased cardiovascular risk, particularly so in individuals with type 2 diabetes, impaired glucose metabolism or insulin resistance. In addition, increased arterial stiffness leads to an increased risk of cardiovascular events. Importantly, stiffness of elastic 
segments (e.g. the carotid artery and ascending aorta) and muscular segments (e.g. the femoral artery and descending aorta) can lead to cardiovascular events independently of each other via distinct pathways. Elastic stiffness is most strongly associated with stroke, whereas muscular stiffness may be most strongly associated with coronary heart disease. Furthermore, arterial stiffness leads to cerebral microvascular damage, which, in turn, can lead to brain dysfunction. Brain dysfunction can manifest itself as cognitive impairment and/or depressive symptoms. Finally, endothelial dysfunction leads to brain dysfunction. This may be through multiple (partially interdependent) pathways, including impairment of the process of neurogenesis, impaired cerebral blood flow regulation, blood-brain barrier dysfunction and microvascular damage.

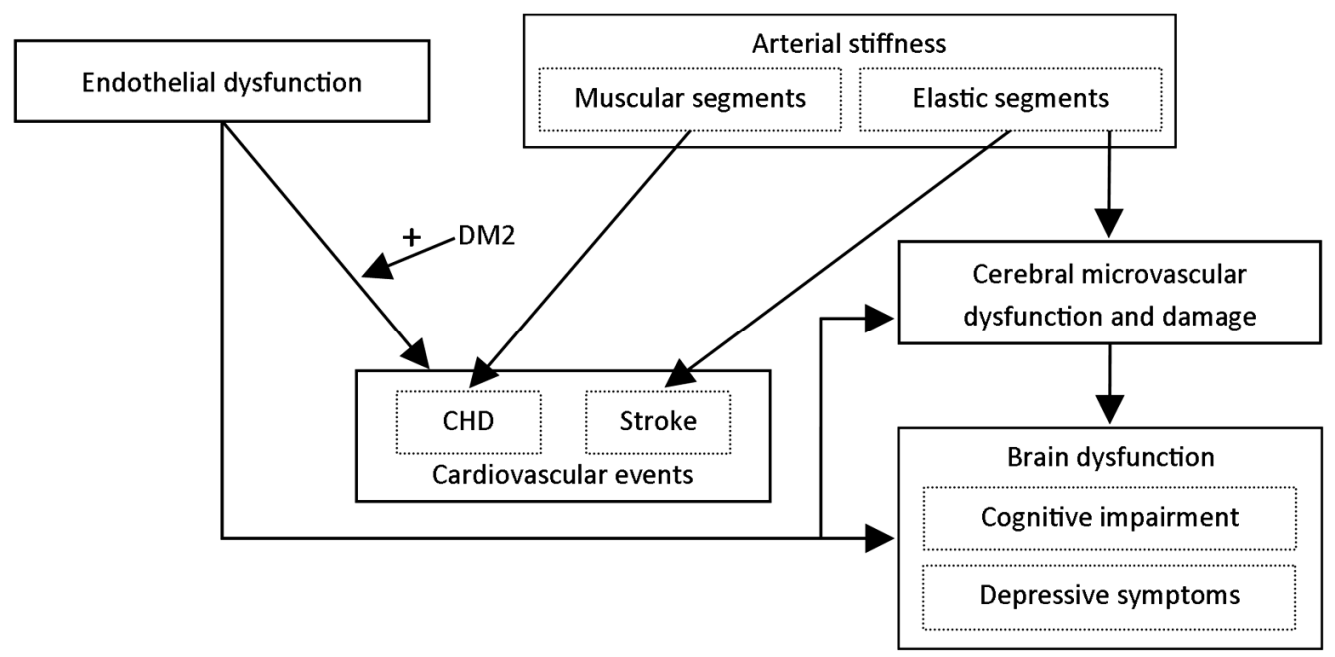

Figure 10.2. Proposed pathophysiological model for the role of arterial stiffness, microvascular dysfunction and endothelial dysfunction in the pathogenesis of cardiovascular events, cognitive impairment and depressive symptoms. DM2 = type 2 diabetes; $\mathrm{CHD}=$ coronary heart disease.

\section{Methodological considerations}

The findings of the present thesis need to be interpreted in light of its methodological limitations. This thesis included a series of observational studies. Associations found in such studies may be biased due to a number of factors, including confounding, overadjustment, selection and information bias. For some observed associations, effect sizes were relatively small. Although such small effects are typical for complex, multifactorial diseases or symptoms, such as cardiovascular disease, cognitive impairment and depressive symptoms, ${ }^{51,52}$ small effects are, in general, difficult to distinguish from bias. ${ }^{53}$ Therefore, replication of these findings in other studies is necessary. Nevertheless, 
in the present thesis many efforts were done to increase the effect-to-bias ratio, or credibility, of the observed associations.

\section{Confounding bias}

Confounding bias is the most important threat to the validity of observational studies. Indeed, it is impossible to exclude the possibility of residual confounding in such studies. ${ }^{19}$ Nevertheless, each study used in the present thesis included an extensive characterization of its participants, which enabled us to adjust for a series of potential confounders in the statistical analysis. In addition, the individual participant data meta-analysis on the association between carotid stiffness and incident cardiovascular events (chapter four) allowed us to re-analyse data of existing studies. We were therefore able to adjust for important potential confounders in each individual dataset. After adjustments, greater carotid stiffness remained associated with a higher incidence of stroke. Furthermore, the results of the systematic review in chapter five showed that in most previous studies the association between greater arterial stiffness on the one hand and markers of cerebral small vessel disease and cognitive impairment on the other remained after adjustment for potential confounders.

\section{Overadjustment bias}

To limit the possibility of confounding bias, we adjusted for several potential confounders in the statistical analyses. However, such adjustments may also lead to bias (overadjustment bias). ${ }^{54}$ Indeed, some of the potential confounders adjusted for in the analyses could, at the same time, be an intermediate factor (i.e. lay in the putative causal pathway) and/or a proximal causal factor (i.e. prior to the determinant in the putative causal pathway). For example, the association between arterial stiffness and cardiovascular events was adjusted for the potential confounder blood pressure. However, blood pressure can, at the same time, also be an intermediate factor (greater arterial stiffness $\rightarrow$ higher blood pressure $\rightarrow$ cardiovascular events) or a proximal causal factor (higher blood pressure $\rightarrow$ greater arterial stiffness $\rightarrow$ cardiovascular events). In general, adjustment for any potential confounder or proximal causal factor is warranted. However, adjustment for a variable that is, at the same time, an intermediate factor would (inevitably) have led to an underestimation of the observed associations. Similarly, adjustment for a proximal causal factor, when measured with a greater precision than the determinant under study, would have led to an underestimation of the observed associations. $^{55}$

\section{Selection bias}

Associations may be biased due to the procedures used to select participants, or factors that influence study participation (selection bias). ${ }^{19}$ For most analyses, we excluded participants with missing data (complete-case analyses). In each study, the number of 
participants with missing data was, however, relatively low. It is therefore unlikely that exclusion of these participants has led to (substantial) bias. Furthermore, in chapters four and six, we applied techniques of imputation (imputation by the expectation maximization method and multiple imputation, respectively) to handle missing data. These techniques can reduce bias and increase precision in the presence of any missing data. ${ }^{56}$ In addition, survival bias (a special case of selection bias) may have affected our results, i.e. it is probable that individuals whom died before the start of the studies were those with the strongest association between vascular dysfunction on the one hand and cardiovascular events, cognitive impairment or depressive symptoms on the other. This may be particularly so for results obtained in The Hoorn and The AGES-Reykjavik Studies, because these studies included relatively older individuals (mean study sample age was 67 and 75 years, respectively). Such survival bias would have led to an underestimation of the observed associations.

\section{Information bias: measures of vascular function}

Non-differential and differential misclassification of individuals may have occurred due to measurement error (information bias). Various measures of vascular function were used. With regard to arterial stiffness, all cohort studies used in the present thesis included measurements on carotid-femoral pulse wave velocity, which is considered the "gold standard" measurement of arterial stiffness. ${ }^{20}$ Furthermore, in The Hoorn Study (chapter three) and most studies included in the meta-analysis on carotid stiffness (chapter four), data on local arterial distensibility were obtained using a validated echotracking ultrasonography technique. ${ }^{20}$

With regard to endothelial dysfunction, a multi-marker approach was used (chapter eight): an extensive set of biomarkers was determined, each reflecting a different facet of endothelial dysfunction, and these biomarkers were summarized into an overall endothelial dysfunction score. ${ }^{57,58}$ The biomarkers used were brachial artery flowmediated dilatation and multiple endothelial-derived circulating biomarkers, including vascular cell adhesion molecule-1, intracellular adhesion molecule-1, endothelial selectin, thrombomodulin and von Willebrand factor. The use of a multi-marker approach has the important merits of statistical efficiency and reduction of the influence of (biological) variability..$^{57,58}$ Importantly, previous studies ${ }^{14,59}$ have shown that such an overall endothelial dysfunction score is strongly associated with a higher cardiovascular event incidence and greater mortality risk. In chapter two, flow-mediated dilatation alone was used to evaluate interaction, with regard to incident cardiovascular events, between endothelial dysfunction and type 2 diabetes. Earlier studies ${ }^{13,14}$ that used circulating endothelial-derived biomarkers to study this interaction found similar results. 
In addition, we evaluated the presence of microvascular dysfunction and damage in the brain (MRI markers of cerebral small vessel disease) and skin (capillaroscopy and laser Doppler flowmetry). Previous studies ${ }^{60-63}$ have demonstrated that MRI markers of cerebral small vessel disease represent both abnormal cerebral microvascular function and structure. In addition, the skin enables direct and non-invasive assessment of microvascular function both at rest and during provocative stimuli. In the present thesis, skin microvascular function was used as a proxy for generalized microvascular function. Indeed, there is substantial evidence ${ }^{46-48}$ that the skin microcirculation is representative of the microcirculation in general.

\section{Information bias: cognitive impairment and depressive symptoms}

The systematic review in chapter five showed that existing evidence of the association between arterial stiffness and cognitive impairment is mainly based on studies that evaluated cognitive impairment or subtle cognitive changes, but not dementia. In addition, in the present thesis, depressive symptoms were assessed by questionnaire and use of antidepressant medication, but not by a structured interview. Therefore, no information was available on clinical depression. The investigation of cognitive impairment or depressive symptoms is important as it contributes to the understanding of the pathogenesis of dementia and clinical depression. However, such impairment or symptoms do not necessarily reflect underlying disease or lead to future disease, and their clinical value is currently not fully understood. ${ }^{64,65}$ Future studies are, therefore, warranted to evaluate whether the observed associations for cognitive impairment and depressive symptoms are also true for dementia and clinical depression. In addition, such studies need to take into account different clinical manifestations of depression. Depression disorders are heterogeneous in terms of symptoms, course and response to treatment. ${ }^{66,67}$ Late-life depression associated with cerebral small vessel disease may be a distinct subtype of depression. ${ }^{68}$ Indeed, some studies ${ }^{69-72}$ (but not all ${ }^{73,74}$ ) found that individuals with vascular-related depression as compared to those with nonvascular depression more often had impairment of executive function and attention, ${ }^{69,70}$ loss of motivation, ${ }^{69,70}$ and a worse response to antidepressant treatment. ${ }^{71,72}$ In addition, it has been $\operatorname{argued}^{75,76}$ that the association between cerebral small vessel disease and depression may be (partially) attributable to apathy. Apathy overlaps with depression, but may be a distinct syndrome, ${ }^{77}$ and this issue requires further study.

\section{Temporality of the observed associations}

Some chapters in this thesis included a cross-sectional data analysis, i.e. the chapters on the association between arterial stiffness or endothelial dysfunction on the one hand and depressive symptoms or skin microvascular function on the other (chapters seven to nine). In addition, the systematic review in chapter five showed that most previous studies on the association between arterial stiffness, cerebral small vessel disease and cognitive 
impairment were cross-sectional by design. We therefore cannot make any conclusions about the temporality of these associations and this issue requires further study.

\section{Future perspectives}

The present thesis provides further evidence for a role of vascular dysfunction in the pathogenesis of cardiovascular disease, cognitive impairment and depressive symptoms. This identifies vascular dysfunction as an important target in the prevention and treatment of these diseases or symptoms. Some of the associations were, however, evaluated in cross-sectional studies only; longitudinal studies are, therefore, needed to assess the temporality of these associations. Ideally, such studies should use an extensive phenotyping approach with detailed measurements on the function and structure of the (micro)vasculature as well as measurements on cardiac and cerebral function, including assessment of dementia and (subtypes of) clinical depression. Such an approach will help to further understand the complex associations between vascular dysfunction and disease. In addition, new epidemiological techniques have become available for (etiologic) observational research, including use of instrumental variables (e.g. Mendelian randomization techniques ${ }^{78}$ ), adjustment for time-dependent confounding, ${ }^{79}$ and estimation of the potential impact of unmeasured confounders. ${ }^{80}$ These techniques may help to further minimize the influence of bias in observational research. Finally, clinical trials are warranted to identify successful (non)pharmacological treatment strategies to counter the adverse effects of vascular dysfunction. Such trials are currently conducted ${ }^{81}$; their results are highly anticipated. 


\section{References}

1. Vaupel JW. Biodemography of human ageing. Nature. 2010;464:536-542.

2. Stehouwer CD, Henry RM, Ferreira I. Arterial stiffness in diabetes and the metabolic syndrome: a pathway to cardiovascular disease. Diabetologia. 2008;51:527-539.

3. Lakatta EG, Levy D. Arterial and cardiac aging: major shareholders in cardiovascular disease enterprises: Part I: aging arteries: a "set up" for vascular disease. Circulation. 2003;107:139-146.

4. Lakatta EG, Levy D. Arterial and cardiac aging: major shareholders in cardiovascular disease enterprises: Part II: the aging heart in health: links to heart disease. Circulation. 2003;107:346-354.

5. Mancia G, Fagard R, Narkiewicz K, et al. 2013 ESH/ESC Guidelines for the management of arterial hypertension: the Task Force for the management of arterial hypertension of the European Society of Hypertension (ESH) and of the European Society of Cardiology (ESC). J Hypertens. 2013;31:1281-1357.

6. Gorelick PB, Scuteri A, Black SE, et al. Vascular contributions to cognitive impairment and dementia: a statement for healthcare professionals from the american heart association/american stroke association. Stroke. 2011;42:2672-2713.

7. Flammer AJ, Anderson T, Celermajer DS, et al. The assessment of endothelial function: from research into clinical practice. Circulation. 2012;126:753-767.

8. Cavalcante JL, Lima JA, Redheuil A, Al-Mallah MH. Aortic stiffness: current understanding and future directions. J Am Coll Cardiol. 2011;57:1511-1522.

9. Emerging Risk Factors C, Di Angelantonio E, Gao P, et al. Lipid-related markers and cardiovascular disease prediction. JAMA. 2012;307:2499-2506.

10. Emerging Risk Factors C, Kaptoge S, Di Angelantonio E, et al. C-reactive protein concentration and risk of coronary heart disease, stroke, and mortality: an individual participant meta-analysis. Lancet. 2010;375:132-140.

11. Gansevoort RT, Correa-Rotter R, Hemmelgarn BR, et al. Chronic kidney disease and cardiovascular risk: epidemiology, mechanisms, and prevention. Lancet. 2013;382:339-352.

12. Lewington S, Clarke R, Qizilbash N, Peto R, Collins R, Prospective Studies C. Age-specific relevance of usual blood pressure to vascular mortality: a meta-analysis of individual data for one million adults in 61 prospective studies. Lancet. 2002;360:1903-1913.

13. Frankel DS, Meigs JB, Massaro JM, et al. Von Willebrand factor, type 2 diabetes mellitus, and risk of cardiovascular disease: the framingham offspring study. Circulation. 2008;118:2533-2539.

14. de Jager J, Dekker JM, Kooy A, et al. Endothelial dysfunction and low-grade inflammation explain much of the excess cardiovascular mortality in individuals with type 2 diabetes: the Hoorn Study. Arterioscler Thromb Vasc Biol. 2006;26:1086-1093.

15. Kim JA, Montagnani M, Koh KK, Quon MJ. Reciprocal relationships between insulin resistance and endothelial dysfunction: molecular and pathophysiological mechanisms. Circulation. 2006;113:18881904.

16. Muris DM, Houben AJ, Schram MT, Stehouwer CD. Microvascular dysfunction is associated with a higher incidence of type 2 diabetes mellitus: a systematic review and meta-analysis. Arterioscler Thromb Vasc Biol. 2012;32:3082-3094.

17. Schalkwijk CG, Stehouwer CD. Vascular complications in diabetes mellitus: the role of endothelial dysfunction. Clin Sci (Lond). 2005;109:143-159.

18. Paneni F, Beckman JA, Creager MA, Cosentino F. Diabetes and vascular disease: pathophysiology, clinical consequences, and medical therapy: part I. Eur Heart J. 2013;34:2436-2443.

19. Rothman KJ, Greenland S, Lash TJ. Modern Epidemiology. Philadelphia: Lippincott Williams And Wilkins; 2008.

20. Laurent S, Cockcroft J, Van Bortel L, et al. Expert consensus document on arterial stiffness: methodological issues and clinical applications. Eur Heart J. 2006;27:2588-2605. 
21. Nichols WW, O'Rourke MF, Vlachopoulos C. McDonald's blood flow in arteries: Theoretical, Experimental and Clinical Principles, 6th edition. London: Hodder Arnold; 2011.

22. Yang EY, Chambless L, Sharrett AR, et al. Carotid arterial wall characteristics are associated with incident ischemic stroke but not coronary heart disease in the Atherosclerosis Risk in Communities (ARIC) study. Stroke. 2012;43:103-108.

23. Selwaness $M$, van den Bouwhuijsen $Q$, Mattace-Raso FU, et al. Arterial stiffness is associated with carotid intraplaque hemorrhage in the general population: the Rotterdam study. Arterioscler Thromb Vasc Biol. 2014;34:927-932.

24. Zureik M, Temmar M, Adamopoulos C, et al. Carotid plaques, but not common carotid intima-media thickness, are independently associated with aortic stiffness. J Hypertens. 2002;20:85-93.

25. Maher E EM, Creane A, Lally C, Kelly DJ. Site specific inelasticity of arterial tissue. J Biomech. 2021;8:1393-1399.

26. Tzourio C, Laurent S, Debette S. Is hypertension associated with an accelerated aging of the brain? Hypertension. 2014;63:894-903.

27. O'Rourke MF, Safar ME. Relationship between aortic stiffening and microvascular disease in brain and kidney: cause and logic of therapy. Hypertension. 2005;46:200-204.

28. Mitchell GF. Effects of central arterial aging on the structure and function of the peripheral vasculature: implications for end-organ damage. J Appl Physiol (1985). 2008;105:1652-1660.

29. Schillaci G, Bilo G, Pucci G, et al. Relationship between short-term blood pressure variability and largeartery stiffness in human hypertension: findings from 2 large databases. Hypertension. 2012;60:369377.

30. Rothwell PM. Limitations of the usual blood-pressure hypothesis and importance of variability, instability, and episodic hypertension. Lancet. 2010;375:938-948.

31. Hughes TM, Kuller LH, Barinas-Mitchell EJ, et al. Arterial Stiffness and beta-Amyloid Progression in Nondemented Elderly Adults. JAMA Neurol. 2014;5:562-568.

32. Zhao C, Deng W, Gage FH. Mechanisms and functional implications of adult neurogenesis. Cell. 2008;132:645-660.

33. Sabayan B, Westendorp RG, Grond J, et al. Markers of endothelial dysfunction and cerebral blood flow in older adults. Neurobiol Aging. 2014;35:373-377.

34. Abbott NJ, Ronnback L, Hansson E. Astrocyte-endothelial interactions at the blood-brain barrier. Nat Rev Neurosci. 2006;7:41-53.

35. Wardlaw JM, Smith C, Dichgans M. Mechanisms of sporadic cerebral small vessel disease: insights from neuroimaging. Lancet Neurol. 2013;12:483-497.

36. Wolinsky H. A proposal linking clearance of circulating lipoproteins to tissue metabolic activity as a basis for understanding atherogenesis. Circ Res. 1980;47:301-311.

37. Mitchell GF, van Buchem MA, Sigurdsson S, et al. Arterial stiffness, pressure and flow pulsatility and brain structure and function: the Age, Gene/Environment Susceptibility--Reykjavik study. Brain. 2011;134:3398-3407.

38. Krishnan KR, Hays JC, Blazer DG. MRI-defined vascular depression. Am J Psychiatry. 1997;154:497-501.

39. Alexopoulos GS, Meyers BS, Young RC, Campbell S, Silbersweig D, Charlson M. 'Vascular depression' hypothesis. Arch Gen Psychiatry. 1997;54:915-922.

40. Pedersen CB, Mors O, Bertelsen A, et al. A comprehensive nationwide study of the incidence rate and lifetime risk for treated mental disorders. JAMA Psychiatry. 2014;71:573-581.

41. Taragano FE, Bagnatti P, Allegri RF. A double-blind, randomized clinical trial to assess the augmentation with nimodipine of antidepressant therapy in the treatment of "vascular depression". Int Psychogeriatr. 2005;17:487-498.

42. Mitchell GF, Vita JA, Larson MG, et al. Cross-sectional relations of peripheral microvascular function, cardiovascular disease risk factors, and aortic stiffness: the Framingham Heart Study. Circulation. 2005;112:3722-3728. 
43. Kim ES, Moon SD, Kim HS, et al. Diabetic peripheral neuropathy is associated with increased arterial stiffness without changes in carotid intima-media thickness in type 2 diabetes. Diabetes Care. 2011;34:1403-1405.

44. Yasuno S, Ueshima K, Oba K, et al. Is pulse pressure a predictor of new-onset diabetes in high-risk hypertensive patients?: a subanalysis of the Candesartan Antihypertensive Survival Evaluation in Japan (CASE-J) trial. Diabetes Care. 2010;33:1122-1127.

45. Giallauria F, Ling SM, Schreiber C, et al. Arterial stiffness and bone demineralization: the Baltimore longitudinal study of aging. Am J Hypertens. 2011;24:970-975.

46. Holowatz LA, Thompson-Torgerson CS, Kenney WL. The human cutaneous circulation as a model of generalized microvascular function. J Appl Physiol (1985). 2008;105:370-372.

47. de Jongh RT, Serne EH, RG IJ, de Vries G, Stehouwer CD. Impaired microvascular function in obesity: implications for obesity-associated microangiopathy, hypertension, and insulin resistance. Circulation. 2004;109:2529-2535.

48. IJzerman RG, de Jongh RT, Beijk MA, et al. Individuals at increased coronary heart disease risk are characterized by an impaired microvascular function in skin. Eur J Clin Invest. 2003;33:536-542.

49. Fegan PG, Tooke JE, Gooding KM, Tullett JM, MacLeod KM, Shore AC. Capillary pressure in subjects with type 2 diabetes and hypertension and the effect of antihypertensive therapy. Hypertension. 2003;41:1111-1117.

50. Sandeman DD, Shore AC, Tooke JE. Relation of skin capillary pressure in patients with insulindependent diabetes mellitus to complications and metabolic control. N Engl J Med. 1992;327:760764.

51. Nissen SE. Biomarkers in cardiovascular medicine: the shame of publication bias. JAMA Intern Med. 2013;173:671-672.

52. Tzoulaki I, Siontis KC, Evangelou E, loannidis JP. Bias in associations of emerging biomarkers with cardiovascular disease. JAMA Intern Med. 2013;173:664-671.

53. Ioannidis JP, Greenland S, Hlatky MA, et al. Increasing value and reducing waste in research design, conduct, and analysis. Lancet. 2014;383:166-175.

54. Schisterman EF CS, Platt RW. Overadjustment bias and unnecessary adjustment in epidemiologic studies. Epidemiology. 2009;20:488-495.

55. Wlazlo N. Novel aspects of insulin resistance and type 2 diabetes mellitus: iron metabolism, the complement system, and liver cirrhosis. Maastricht, Maastricht University; 2014.

56. Sterne JA, White IR, Carlin JB, et al. Multiple imputation for missing data in epidemiological and clinical research: potential and pitfalls. BMJ. 2009;338:b2393.

57. van Bussel BC, Henry RM, Schalkwijk CG, Dekker JM, Nijpels G, Stehouwer CD. Low-grade inflammation, but not endothelial dysfunction, is associated with greater carotid stiffness in the elderly: the Hoorn Study. J Hypertens. 2012;30:744-752.

58. van Bussel BC, Schouten F, Henry RM, et al. Endothelial dysfunction and low-grade inflammation are associated with greater arterial stiffness over a 6-year period. Hypertension. 2011;58:588-595.

59. Astrup AS, Tarnow L, Pietraszek L, et al. Markers of endothelial dysfunction and inflammation in type 1 diabetic patients with or without diabetic nephropathy followed for 10 years: association with mortality and decline of glomerular filtration rate. Diabetes Care. 2008;31:1170-1176.

60. Fisher CM. Capsular infarcts: the underlying vascular lesions. Arch Neurol. 1979;36:65-73.

61. Joutel A, Monet-Lepretre M, Gosele C, et al. Cerebrovascular dysfunction and microcirculation rarefaction precede white matter lesions in a mouse genetic model of cerebral ischemic small vessel disease. J Clin Invest. 2010;120:433-445.

62. Wardlaw JM, Doubal F, Armitage $P$, et al. Lacunar stroke is associated with diffuse blood-brain barrier dysfunction. Ann Neurol. 2009;65:194-202.

63. Young VG, Halliday GM, Kril JJ. Neuropathologic correlates of white matter hyperintensities. Neurology. 2008;71:804-811. 
64. Le Couteur DG, Doust J, Creasey H, Brayne C. Political drive to screen for pre-dementia: not evidence based and ignores the harms of diagnosis. BMJ. 2013;347:f5125.

65. Thombs BD, de Jonge $\mathrm{P}$, Coyne JC, et al. Depression screening and patient outcomes in cardiovascular care: a systematic review. JAMA. 2008;300:2161-2171.

66. Antonijevic IA. Depressive disorders -- is it time to endorse different pathophysiologies? Psychoneuroendocrinology. 2006;31:1-15.

67. Ghaemi SN, Vohringer PA. The heterogeneity of depression: an old debate renewed. Acta Psychiatr Scand. 2011;124:497.

68. Roman GC. Vascular depression: An archetypal neuropsychiatric disorder. Biol Psychiatry. 2006;60:1306-1308.

69. Taylor WD, Aizenstein HJ, Alexopoulos GS. The vascular depression hypothesis: mechanisms linking vascular disease with depression. Mol Psychiatry. 2013;18:963-974.

70. Rapp MA, Dahlman K, Sano M, Grossman HT, Haroutunian V, Gorman JM. Neuropsychological differences between late-onset and recurrent geriatric major depression. Am J Psychiatry. 2005;162:691-698.

71. Sneed JR, Culang-Reinlieb ME, Brickman AM, et al. MRI signal hyperintensities and failure to remit following antidepressant treatment. J Affect Disord. 2011;135:315-320.

72. Sheline YI, Pieper CF, Barch DM, et al. Support for the vascular depression hypothesis in late-life depression: results of a 2-site, prospective, antidepressant treatment trial. Arch Gen Psychiatry. 2010;67:277-285.

73. Baldwin $\mathrm{R}$, Jeffries $\mathrm{S}$, Jackson $\mathrm{A}$, et al. Treatment response in late-onset depression: relationship to neuropsychological, neuroradiological and vascular risk factors. Psychol Med. 2004;34:125-136.

74. Naarding $\mathrm{P}$, Tiemeier $\mathrm{H}$, Breteler $\mathrm{MM}$, et al. Clinically defined vascular depression in the general population. Psychol Med. 2007;37:383-392.

75. Grool AM, Gerritsen L, Zuithoff NP, Mali WP, van der Graaf Y, Geerlings MI. Lacunar infarcts in deep white matter are associated with higher and more fluctuating depressive symptoms during three years follow-up. Biol psychiatry. 2013;73:169-176.

76. Ligthart SA, Richard E, Fransen NL, et al. Association of vascular factors with apathy in communitydwelling elderly individuals. Arch Gen Psychiatry. 2012;69:636-642.

77. Marin RS. Apathy: a neuropsychiatric syndrome. J Neuropsychiatry Clin Neurosci. 1991;3:243-254.

78. Didelez V, Sheehan N. Mendelian randomization as an instrumental variable approach to causal inference. Stat Methods Med Res. 2007;16:309-330.

79. Daniel RM, Cousens SN, De Stavola BL, Kenward MG, Sterne JA. Methods for dealing with timedependent confounding. Stat Med. 2013;32:1584-1618.

80. Groenwold RH, Nelson DB, Nichol KL, Hoes AW, Hak E. Sensitivity analyses to estimate the potential impact of unmeasured confounding in causal research. Int J Epidemiol. 2010;39:107-117.

81. Laurent S, Briet M, Boutouyrie P. Arterial stiffness as surrogate end point: needed clinical trials. Hypertension. 2012;60:518-522. 
General discussion | 233 


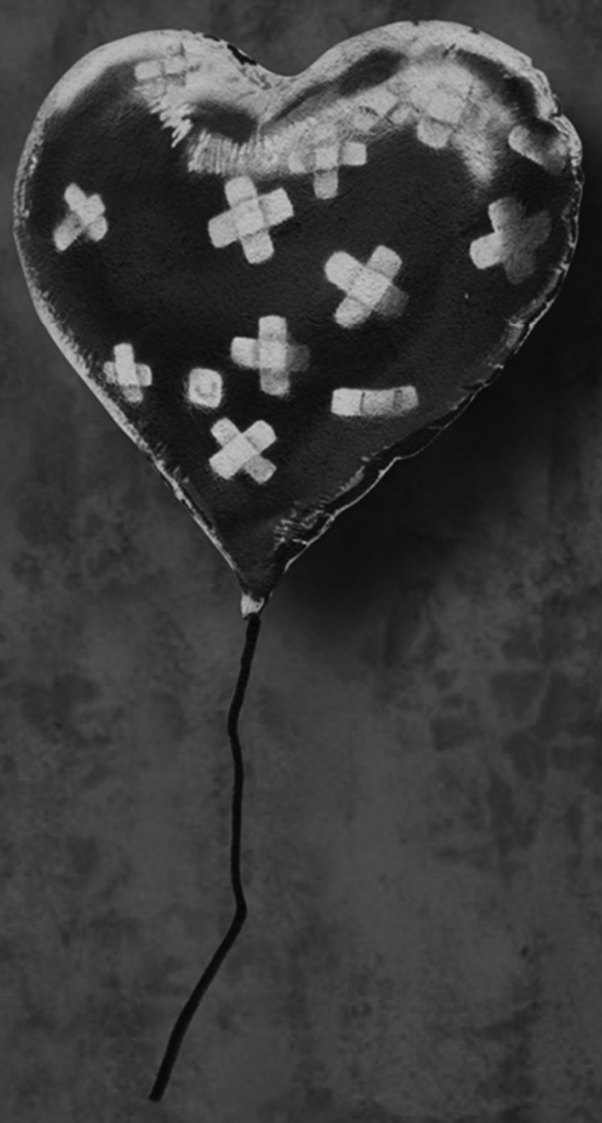

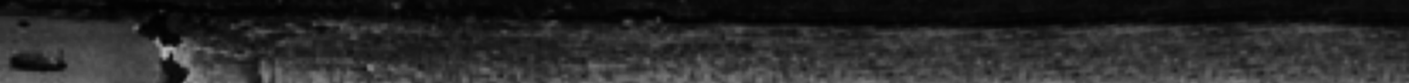
1. 1. 1. 


\section{Chapter 11}

Summary

Nederlandstalige samenvatting

List of publications

Valorization addendum

Dankwoord

Curriculum Vitae 



\section{Summary}

Emerging evidence indicates that vascular dysfunction is an important pathway through which ageing and other risk factors, such as type 2 diabetes and obesity, can cause diseases of both the heart and brain. Vascular dysfunction includes dysfunction of large arteries (due to arterial stiffness), the microcirculation (microvascular dysfunction) and endothelium (endothelial dysfunction). The present thesis sought to further investigate, in a series of epidemiological studies, the role of vascular dysfunction in the pathogenesis cardiovascular disease, cognitive impairment and depressive symptoms.

Chapter one provides an overview of the underlying mechanisms of vascular dysfunction and includes a description of the cohort studies used in the present thesis.

In chapter two, we showed the presence of interaction, i.e. synergy, with regard to cardiovascular event risk, between, on the one hand, endothelial dysfunction, as defined by impaired flow-mediated dilatation, and, on the other, type 2 diabetes, impaired glucose metabolism and insulin resistance.

In chapter three, we showed that greater stiffness of the carotid and femoral arteries is associated with a higher incidence of cardiovascular events and greater all-cause mortality, independently of cardiovascular factors, and independently of other stiffness indices, including carotid-femoral pulse wave velocity.

In chapter four, we did a systematic review and an aggregate data and an individual participant data meta-analysis. We showed that greater carotid stiffness is associated with a higher stroke incidence, but not with incident coronary heart disease events. The association between carotid stiffness and incident stroke was independent of cardiovascular risk factors, and independent of carotid-femoral pulse wave velocity. In addition, we found that carotid stiffness modestly improves risk prediction of stroke beyond Framingham stroke risk score factors and carotid-femoral pulse wave velocity.

In chapter five, we conducted a systematic review and an aggregate data meta-analysis and we showed that a consistent association exists between greater arterial stiffness on the one hand and markers of cerebral small vessel disease and cognitive impairment on the other.

In chapter six, we found that various magnetic resonance imaging markers of baseline and progression over time of cerebral small vessel disease are independently and strongly 
associated with a higher incidence of depressive symptoms. In addition, we showed that cerebral small vessel disease in the deep brain region is, as compared to other regions, more strongly associated with a higher incidence of depressive symptoms.

In chapter seven, we showed that greater arterial stiffness is associated with more (severe) depressive symptoms and that this association is in part mediated by higher white matter hyperintensity volume and subcortical infarcts.

In chapter eight, we found that endothelial dysfunction, as quantified by an array of endothelial-derived circulating biomarkers and flow-mediated dilatation, is independently associated with more (severe) depressive symptoms.

In chapter nine, we showed that arterial stiffness is not associated with skin microvascular function, irrespective of the presence of type 2 diabetes.

Finally, in chapter ten we discussed the key findings of the present thesis and their clinical implications. In addition, methodological considerations and directions for future research were addressed. 
Summary | 239 



\section{Nederlandstalige samenvatting}

De levensverwachting in Nederland is in de afgelopen 100 jaar sterk verbeterd en zal nog verder toenemen in de komende decennia. Veroudering geeft een verhoogde kans op het krijgen van ziekten van het hart en de hersenen, zoals een hartinfarct, hartfalen en een beroerte (ook wel hart- en vaatziekten genoemd) en dementie en depressie. De last van deze ziekten is hoog, voor zowel patienten, mantelzorgers als de maatschappij. Het is daarom belangrijk om de oorzaken van deze ziekten te onderzoeken. Zulke oorzaken zijn mogelijke aangrijpingspunten voor nieuwe behandelingen. Eerder onderzoek laat zien dat verslechterde functie van de vaten in het lichaam (vaatdisfunctie) mogelijk een centrale rol inneemt bij het ontstaan van ziekten van het hart en de hersenen. Het doel van dit proefschrift was om de rol van vaatdisfunctie verder te onderzoeken in het ontstaan van hart- en vaatziekten, cognitieve disfunctie en depressieve klachten.

Wij hebben gekeken naar disfunctie van drie verschillende onderdelen van het vaatstelsel:

1) van de grote slagaders (disfunctie door vaatverstijving),

2) van de kleinste vaatjes in het lichaam - de microcirculatie (microvasculaire disfunctie), en

3) van de laag cellen die de binnenkant vormt van alle bloedvaten - het endotheel (endotheeldisfunctie).

Hier volgt eerst een beschrijving van de mechanismen waardoor vaatdisfunctie kan bijdragen aan het ontstaan van hart- en vaatziekten, cognitieve disfunctie en depressieve klachten. Daarna worden de belangrijkste bevindingen van dit proefschrift beschreven. Voor dit proefschrift werd gebruik gemaakt van verschillende studies, uitgevoerd in grote groepen mensen (epidemiologisch onderzoek).

\section{Vaatdisfunctie als oorzaak van ziekten van het hart en de hersenen: onderliggende mechanismen}

Met de leeftijd en onder invloed van verschillende risicofactoren, zoals suikerziekte en overgewicht, neemt de elasticiteit van bloedvaten af: er is sprake van vaatverstijving. Vaatverstijving heeft een aantal schadelijke effecten. Het leidt tot veranderingen in de bloeddruk: de bovendruk neemt toe, terwijl de onderdruk juist afneemt. Hierdoor neemt het verschil tussen de boven- en onderdruk, de polsdruk, sterk toe. Deze verhoogde polsdruk heeft als gevolg dat het hart veel harder moet werken om bloed door het lichaam te pompen. Tegelijkertijd neemt de bloeddoorstroming van het hart af, omdat deze afhankelijk is van de hoogte van de onderdruk. Door deze veranderingen ontstaat er 
zuurstoftekort van het hart en hartfalen. Daarnaast kan de verhoogde polsdruk doordringen tot in de microcirculatie en daar schade toebrengen. De microcirculatie van de hersenen is extra kwetsbaar voor deze verhoogde polsdruk. Microvasculaire disfunctie in de hersenen kan leiden tot het optreden van beroertes, cognitieve disfunctie (waaronder dementie) en mogelijk ook depressieve klachten.

Ook endotheeldisfunctie kan op verschillende manieren bijdragen aan het ontstaan van ziekten van het hart en de hersenen. Endotheeldisfunctie is een oorzaak van aderverkalking. Aderverkalking kan leiden afsluiting van de vaten rondom het hart en de hersenen. Daarnaast leidt microvasculaire disfunctie en endotheeldisfunctie tot een gestoorde regulatie van de bloeddoorstroming en tot lekkage van bloedvaten.

\section{Belangrijkste bevindingen}

Endotheeldisfunctie geeft een verhoogd risico op hart- en vaatziekten, vooral bij mensen met suikerziekte

Eerder onderzoek heeft laten zien dat endotheeldisfunctie een hoger risico geeft op harten vaatziekten. $\mathrm{Er}$ is gesuggereerd dat mensen met suikerziekte type 2 (type 2 diabetes) extra gevoelig zijn voor de schadelijke effecten van endotheeldisfunctie, maar hiervoor was tot nu toe onvoldoende bewijs. In dit proefschrift hebben wij dit onderzocht in de Hoorn Studie, een onderzoek uitgevoerd in Hoorn. In deze studie zijn bij bijna 500 deelnemers metingen gedaan van de werking van het endotheel. Vervolgens is elke deelnemer in de loop van de tijd gevolgd om te kijken of hij/zij een hart- of vaatziekte kreeg. De resultaten lieten zien dat mensen met suikerziekte inderdaad extra gevoelig zijn: bij deze mensen gaf een slechtere functie van het endotheel een veel hoger risico op het krijgen van hart- en vaatziekten dan bij mensen zonder suikerziekte. Bovendien was dit verhoogde risico al aanwezig bij mensen met een voorstadium van suikerziekte, ook wel pre-diabetes genoemd. Een verklaring voor deze bevindingen is dat suikerziekte en endotheeldisfunctie twee mechanismen zijn die elkaar kunnen versterken. Behalve dat suikerziekte leidt tot endotheeldisfunctie, leidt endotheeldisfunctie ook tot suikerziekte. Verbeteren van de werking van het endotheel is daarmee een belangrijk nieuw aangrijpingspunt voor de behandeling gericht op het voorkomen van hart- en vaatziekten bij mensen met suikerziekte.

Stijfheid van zowel de hals-, lies- als lichaamsslagader geeft een verhoogde kans op harten vaatziekten

Eerder onderzoek heeft aangetoond dat mensen met toegenomen stijfheid van de lichaamsslagader een hoger risico hebben op het krijgen van hart- en vaatziekten. Of verstijving van andere slagaders dan de lichaamsslagader ook een verhoogd risico geeft, was tot nu toe onduidelijk. In de Hoorn Studie konden we deze verbanden onderzoeken, 
omdat in deze studie ook de stijfheid van verschillende slagaders zijn gemeten met behulp van echo onderzoek. De belangrijkste bevinding van deze studie was dat, naast verstijving van de lichaamsslagader, ook verstijving van de hals- én liesslagader een verhoogd risico geeft op het krijgen van hart- en vaatziekten. Interessant was dat dit verhoogde risico bestond voor elke slagader, onafhankelijk of er sprake was van toegenomen stijfheid van de andere slagaders. We hebben vervolgens de rol van verstijving van de halsslagader verder onderzocht door gegevens te combineren van verschillende studies met in totaal meer dan 4000 mensen. In deze studies bleek dat verstijving van de halsslagader vooral het risico verhoogd op het krijgen van een beroerte. Deze resultaten suggereren dat behandelingen gericht op verlaging van de stijfheid van verschillende vaten het optreden van hart- en vaatziekten kunnen voorkomen. Bovendien kan het meten van de stijfheid van de verschillende vaten mogelijk helpen om het risico van een persoon op het krijgen van hart- en vaatziekten beter te voorspellen.

\section{Vaatdisfunctie draagt bij aan het ontstaan van cognitieve disfunctie en depressieve klachten}

Eerder onderzoek suggereert dat vaatdisfunctie kan bijdragen aan het ontstaan van verschillende hersenziekten, onder andere dementie en depressie. In dit proefschrift hebben we deze relaties verder onderzocht. Een systematische literatuurstudie liet zien dat eerdere studies inderdaad een relatie hebben gevonden tussen een verhoogde vaatstijfheid enerzijds en de aanwezigheid van microvasculaire hersenschade en cognitieve disfunctie anderzijds. Vervolgens hebben we onderzocht of microvasculaire hersenschade ook leidt tot depressieve klachten. Dit hebben we onderzocht in de AGESReykjavik Studie, een onderzoek uitgevoerd bij bijna 2000 oudere mensen in Reyjavik, IJsland. In deze studie zijn hersenscans gemaakt om de aanwezigheid van microvasculaire hersenschade vast te stellen. De resultaten lieten zien dat microvasculaire hersenschade inderdaad een verhoogd risico geeft op het krijgen van depressieve klachten. Vooral hersenschade gelokaliseerd in gebieden betrokken bij de regulatie van emotie en gevoel (de diepe hersenstructuren) hing sterk samen met het krijgen van depressieve klachten. In de AGES-Reykjavik Studie konden we ook de relatie onderzoeken tussen vaatstijfheid en depressie. In deze studie was vaatstijfheid geassocieerd met de aanwezigheid van depressieve klachten, en dit verband verliep deels via microvasculaire hersenschade. Ook in de Hoorn Studie hebben we deze relaties onderzocht. In deze studie bleek dat ook endotheeldisfunctie samenhangt met de aanwezigheid van meer en ernstigere depressive klachten. Deze verschillende studies geven daarmee meer bewijs voor een rol van vaatdisfunctie in het optreden van cognitieve disfunctie en depressieve klachten. Dit betekent dat de behandeling gericht op verlaging van vaatstijfheid en verbetering van de werking van de microcirculatie en het endotheel een rol kan spelen bij de preventie en behandeling van dementie en depressie. 
De relatie tussen vaatstijfheid en microvasculaire disfunctie is geen universeel fenomeen

$\mathrm{Er}$ is gesuggereerd dat vaatstijfheid ook microvasculaire disfunctie kan veroorzaken in andere organen dan de hersenen (een universeel fenomeen). Met behulp van gegevens van de Maastricht Studie, een groot onderzoek uitgevoerd in Maastricht, konden we dit onderzoeken. In de Maastricht Studie zijn uitgebreide metingen gedaan van de microcirculatie in de huid. Uit deze studie bleek echter dat vaatstijfheid niet geassocieerd was met microvasculaire functie van de huid. Vervolgens hebben we deze relaties ook onderzocht in de SUVIMAX2 Studie, een onderzoek uitgevoerd in Parijs. Ook in deze studie was er geen verband tussen vaatstijfheid en microvasculaire functie gemeten in de huid. Dit suggereert dat de relatie tussen vaatstijfheid en microvasculaire disfunctie geen universeel fenomeen is. Het is waarschijnlijk dat vaatstijfheid alleen de microcirculatie beschadigt in organen die hier extra kwetsbaar voor zijn, zoals de hersenen.

\section{Conclusie}

Dit proefschrift laat zien dat vaatdisfunctie een belangrijke rol speelt in het ontstaan van hart- en vaatziekten, cognitieve disfunctie en depressieve klachten. In figuur 10.3 zijn de belangrijkste bevindingen van dit proefschrift schematisch weergegeven. Op basis van deze bevindingen kan vervolgonderzoek worden gedaan dat zich richt op de vraag of behandeling van vaatdisfunctie inderdaad helpt om hart- en vaatziekten, dementie en depressie te voorkomen.

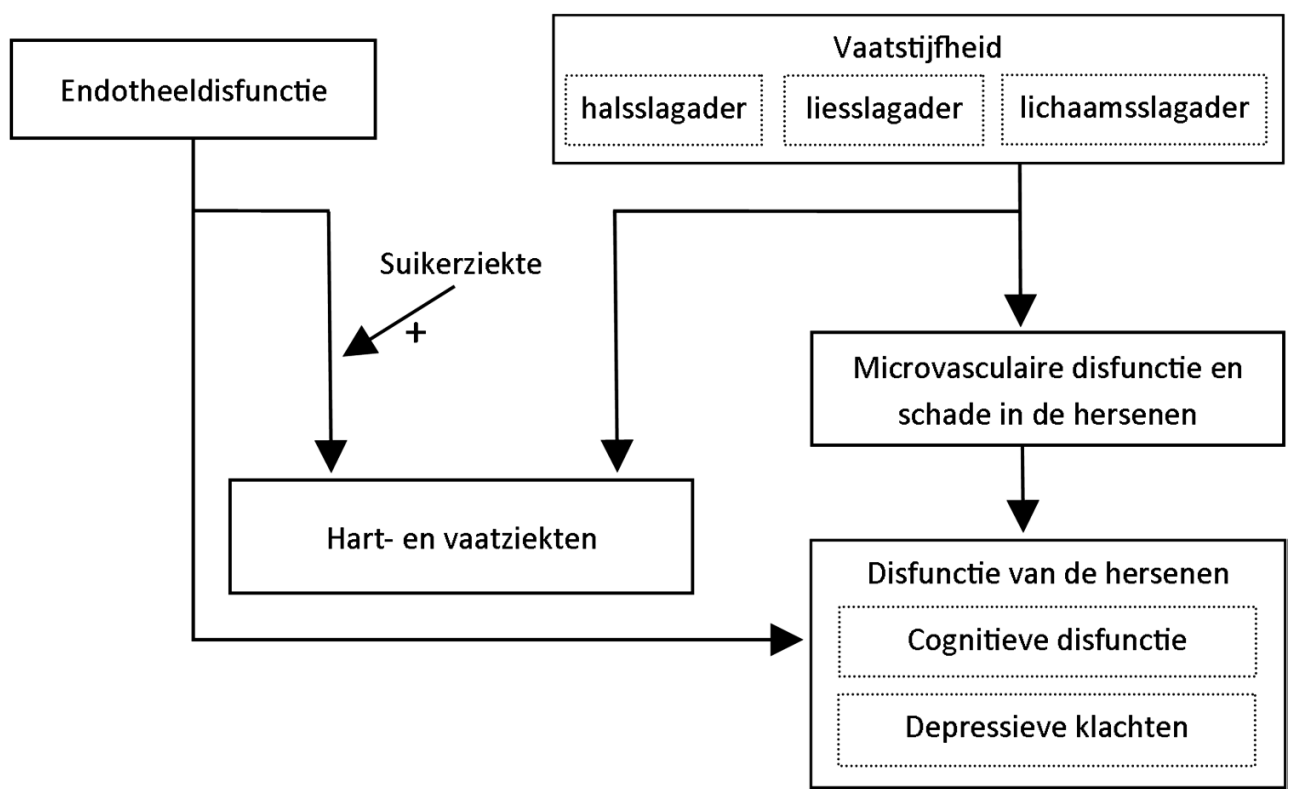

Figuur 11.1. Schematische weergave van de belangrijkste bevindingen van dit proefschrift. 
Nederlandstalige samenvatting | 245 



\section{List of publications}

\section{Thesis}

1. van Sloten TT, Henry RM, Dekker JM, Nijpels G, Unger T, Schram MT, Stehouwer CD. Endothelial dysfunction plays a key role in increasing cardiovascular risk in type 2 diabetes: The Hoorn Study. Hypertension. 2014;64:1299-1305

2. van Sloten TT, Schram MT, van den Hurk K, Dekker JM, Nijpels G, Henry RM, Stehouwer $C D$. Local stiffness of the carotid and femoral artery is associated with incident cardiovascular events and all-cause mortality: The Hoorn Study. J Am Coll Cardiol. 2014;63:1739-1747

3. van Sloten TT, Sedaghat S, Laurent S, London GM, Pannier B, Ikram MA, Kavousi M, Mattace-Raso F, Franco OH, Boutouyrie P, Stehouwer CD. Carotid stiffness is associated with incident stroke: a systematic review and meta-analysis. Submitted

4. van Sloten TT, Protogerou AD, Henry RM, Schram MT, Launer LJ, Stehouwer CD. Association between arterial stiffness, cerebral small vessel disease and cognitive impairment: a systematic review and meta-analysis. Neurosci Biobehav Rev. Accepted for publication

5. van Sloten TT, Sigurdsson S, van Buchem MA, Phillips CL, Jonsson PV, Ding J, Schram MT, Harris TB, Gudnason V, Launer L. Cerebral small vessel disease and association with a higher incidence of depressive symptoms in a general elderly population: The AGESReykjavik Study. Am J Psychiatry. Accepted for publication

6. van Sloten TT, Mitchell GF, Sigurdsson S, van Buchem MA, Jonsson PV, Garcia ME, Harris TB, Henry RM, Levey AS, Stehouwer CD, Gudnason V, Launer L. Arterial stiffness, depressive symptoms and mediation by cerebral small vessel disease: The AGES-Reykjavik Study. Submitted

7. van Sloten TT, Schram MT, Adriaanse MC, Dekker JM, Nijpels G, Teerlink T, Scheffer PG, Pouwer F, Schalkwijk CG, Stehouwer CD, Henry RM. Endothelial dysfunction is associated with a greater depressive symptom score in a general elderly population: The Hoorn Study. Psychol Med. 2014;44:1403-1416 
8. van Sloten TT, Czernichow S, Houben AJ, Protogerou AD, Henry RM, Muris DM, Schram MT, Sep SJ, Dagnelie PC, van der Kallen CJ, Schaper NC, Blacher J, Hercberg S, Levy BI, Stehouwer CD. Association between arterial stiffness and skin microvascular function: The SUVIMAX2 Study and The Maastricht Study. Am J Hypertens. Accepted for publication

\section{Other}

9. van Sloten TT, Stehouwer CD. No need to change guidelines for diabetic retinopathy and renin-angiotensin system inhibitors. Lancet Diabetes Endocrinol. Accepted for publication

10. Protogerou AD, van Sloten TT, Henry RM, Dekker JM, Nijpels G, Stehouwer CD. Pulse pressure measured at the level of the femoral artery, but not at the level of the aorta, carotid and brachial arteries, is associated with the incident of coronary heart disease events in a population with a high prevalence of type 2 diabetes and impaired glucose metabolism: The Hoorn Study. Artery Res. 2015;9:19-26

11. Tai C, Sun Y, Dai N, Xu D, Chen W, Wang J, Protogerou AD, van sloten TT, Blacher J, Safar ME, Zhang Y, Xu Y. Prognostic significance of visit-to-visit blood pressure variability: a meta-analysis of 77,299 patients. J Clin Hypertens. 2015;17:107-115

12. Wijnands JM, Boonen A, van Sloten TT, Dagnelie PC, Stehouwer CD, van der Linden S, Arts IC. Uric acid is not associated with aortic, carotid, or femoral stiffness: The Maastricht Study. J Hypertens. Accepted for publication

13. Geijselaers SL, Sep SJ, Schram MT, van Boxtel M, van Sloten TT, Henry RM, Reesink KD, Kroon AA, Koster A, Schaper NC, Dagnelie PC, van der Kallen CJ, Biessels GJ, Stehouwer CD. Carotid stiffness is associated with cognitive dysfunction in individuals with and without type 2 diabetes: The Maastricht Study. Submitted.

14. Protogerou $A D$, van Sloten TT, Stehouwer $C D$. Aortic stiffness and incident hypertension. JAMA. 2013;309:29-30

15. van Sloten TT, Pijpers E, Stehouwer CD, Brouwers MC. Metformin-associated lactic acidosis in a patient with normal kidney function. Diabetes Res Clin Pract. 2012;96:e57-58

16. van Sloten TT, Savelberg HH, Duimel-Peeters IG, Meijer K, Henry RM, Stehouwer CD, Schaper NC. Peripheral neuropathy, decreased muscle strength and obesity are strongly associated with walking in persons with type 2 diabetes without manifest mobility limitations. Diabetes Res Clin Pract. 2011;91:32-39 
17. van Sloten TT, Friederichs SA, Huijberts MS, Schaper NC. Diabetische voet: nieuwe inzichten in pathofysiologie en behandeling. Ned Tijdschr Geneeskd. 2008;152:2400-2405 



\section{Valorization addendum}

One of the main tasks of Universities is to ensure that research findings impact society, i.e. valorization. This addendum discusses the valorization potential of several main findings of the present thesis: the findings that vascular brain damage is an important risk factor for late-life depression, and that arterial stiffness and microvascular and endothelial dysfunction may lead to vascular brain damage.

\section{Relevance of the findings}

Life expectance in the Netherlands and much of the rest of the world has dramatically increased and will continue to do so in the next decades. Depression and depressive symptoms are often present in older individuals, and pose an enormous emotional and economic burden to individuals, families and health care systems. There is, thus, a growing need to understand their causes. The investigation of such causes will help to identify targets for prevention and treatment of late-life depression. The present thesis strongly suggests that damage of the small arteries in the brain (cerebral small vessel disease) is an important risk factor for late-life depression. The underlying mechanism is that vascular damage may lead to depression via disruption of brain structures involved in mood regulation.

The identification of vascular brain damage as a potential cause of late-life depression will have societal impact. Indeed, this finding suggests that efforts at favourably influencing vascular damage can have significant public health implications via prevention and treatment of depression. However, before this finding can be translated into treatment strategies, further research is warranted. Currently, it is incompletely understood which factors contribute to cerebral small vessel disease, and whether such factors can be therapeutic targets for late-life depression. This addendum therefore includes a proposal for further observational studies and clinical trials, and discusses how the findings of this research can lead to new prevention and treatment strategies of depression.

\section{Future research: observational studies}

The findings presented in this thesis suggest that several (interrelated) processes may lead to cerebral small vessel disease and depression, including arterial stiffness and microvascular and endothelial dysfunction. These findings were, however, based on crosssectional studies. This precludes any conclusions about a temporal association of arterial stiffness and microvascular and endothelial dysfunction to cerebral small vessel disease and depression. Furthermore, in the present thesis information was available on depressive symptoms only, but not on clinical depression. The investigation of depressive 
symptoms is important as it contributes to understanding the pathogenesis of depression. However, depressive symptoms do not necessarily reflect underlying disease or lead to future disease, and their clinical value is currently not fully understood. Future studies are, therefore, warranted to evaluate whether the observed associations for depressive symptoms are also true for clinical depression.

In view of the above, we propose to use cross-sectional and longitudinal data of The Maastricht Study to evaluate the association between arterial stiffness and endothelial and microvascular dysfunction on the one hand and cerebral small vessel disease and depression on the other. The Maastricht Study is a large, longitudinal observational study conducted in Maastricht and is expected to become one of the most extensive phenotyping studies worldwide. The Maastricht Study focuses on the investigation of causes of type 2 diabetes and other chronic diseases, including depression. The study uses state-of-the-art imaging techniques and extensive biobanking to determine the health status in a population-based cohort of 10,000 individuals that is enriched with individuals with type 2 diabetes aged between 40 and 75 years. Enrolment of participants of The Maastricht Study started in November 2010 and is anticipated be complete in the end of 2015 / beginning of 2016. After this period, a follow-up examination round will be done. The results of cross-sectional and longitudinal data are expected to be available in 2016 and 2020, respectively. In this study, depressive symptoms and depression are evaluated by questionnaire and by a structured interview conducted by trained investigators. In addition, participants undergo a magnetic resonance imaging (MRI) investigation of the brain to evaluate the presence of cerebral small vessel disease. Arterial stiffness, in turn, is determined at different sites and segments, and with use of different techniques. These include the determination of segmental aortic stiffness with use of carotid-femoral pulse wave velocity, and assessment of local carotid, femoral and brachial artery stiffness by vascular ultrasonography. In addition, detailed measurements are done of the function and structure of the microvasculature and endothelium of both the skin (e.g. capillaroscopy and Laser Doppler flowmetry) and retina (e.g. funduscopy and dynamic retinal vessel analyzer). Furthermore, endothelial dysfunction is assessed by the determination of endothelial-derived blood biomarkers.

An important merit of The Maastricht Study is the oversampling of individuals with type 2 diabetes. This allows investigating any potential contrast between individuals with and without type 2 diabetes in the association between arterial stiffness and microvascular and endothelial dysfunction and cerebral small vessel disease. Individuals with type 2 diabetes have a greatly increased risk of cerebral small vessel disease and depression, possibly in part because these individuals may be particularly prone to the detrimental effects of increased arterial stiffness on the cerebral vasculature. In the next years, we will apply for research grants to conduct the research as outlined above. 


\section{Future studies: randomized clinical trials}

In addition to observational studies, future large (multi-centre) randomized clinical trials are needed that evaluate the effects of favourably influencing arterial stiffness and microvascular and endothelial dysfunction. Such trials need to take into account the potential beneficial treatment effects on depression. For instance, depressive symptoms can be used as a secondary end point and/or the effects of treatment on depressive symptoms can be evaluated in a substudy of these trials. Several interventions are available that have a favourable influence on arterial stiffness and endothelial and microvascular dysfunction, including lifestyle modifications, such as weight loss, increased physical activity and dietary modifications, and drugs, such as (long-term) blockade of angiotensin receptor- 1 antagonists and angiotensin receptor-2 agonists. These drugs may lower arterial stiffness, possibly beyond any blood-pressure lowering effects. Currently, no trials on arterial stiffness and microvascular and endothelial dysfunction take into account depression or depressive symptoms. We will try to create awareness of this issue among researchers in the field via publication of articles in peer-reviewed journals on this topic and via presentations at scientific meetings, such as the meetings of the Artery Society and the European Society of Hypertension.

\section{New treatment strategies for late-life depression}

Currently, treatment for almost all older patients with depression or depressive symptoms includes antidepressant medication and/or psychotherapy. The findings of the present thesis, if confirmed in The Maastricht Study and clinical trials, indicate that favourably influencing arterial stiffness and microvascular and endothelial dysfunction are new strategies for prevention and treatment of depression, in particular in older individuals. It is, however, likely that only a subset of older patients with depression have vascularrelated disease. Determination of arterial stiffness (e.g. carotid-femoral pulse wave velocity) or endothelial dysfunction (e.g. brachial artery flow-mediated dilatation) in older patients may help to select these individuals, which could help guide treatment choices and tailor interventions to the individual patient. Thereby, the identification and treatment of vascular-related depression can be a first step toward personalized medicine in (geriatric) psychiatry. 



\section{Dankwoord}

Als $u$ het hele proefschrift gelezen heeft (en waarom zou ik daaraan twijfelen) dan zal het u niet verbazen dat dit proefschrift tot stand is gekomen door de inzet van velen. Graag wil ik een aantal personen in het bijzonder bedanken.

Mijn promotor, prof. dr. C.D.A. Stehouwer.

Beste Coen, ik heb in de afgelopen vier jaar onzettend veel van je geleerd. Je scherpe en glasheldere formuleringen (in manuscripten én emails én tijdens discussies), betrouwbaarheid, hoge (wetenschappelijke) integriteit en streven naar perfectie werken enorm inspirerend. Het is een grote eer om één van jouw promovendi te mogen zijn.

Mijn co-promotoren, dr. R.M.A. Henry en dr. M.T. Schram.

Beste Ronald, tijdens mijn promotie heb ik onze samenwerking als zeer waardevol ervaren. Je positieve en constructieve houding en je enthousiasme voor de wetenschap zijn aanstekelijk en onvervangbaar. Je "schrijfsessies" zijn inmiddels een begrip onder je promovendi.

Beste Miranda, mede dankzij jou heb ik mij bij De Maastricht Studie altijd thuis gevoeld. Je was altijd aanspreekbaar, beoordeelt manuscripten kritisch en regelt snel praktische zaken.

De leden van de beoordelingscomissie.

Prof. dr. H.A.J. Struijker-Boudier, prof. dr. R.J. van Oostenbrugge, prof. dr. B.W.J.H. penninx en dr. K.D. Reesink: bedankt voor het beoordelen van mijn proefschrift. Dear prof. dr. J.K. Cruickshank, thank you for your participation in the Assessment Committee of my thesis.

Alle medewerkers van De Maastricht Studie en de afdeling Interne Geneeskunde.

Mijn collega promovendi: allereerst Marcelle, José en Dennis. Jullie waren de eerste promovendi bij De Maastricht Studie en hebben het meeste werk verricht om de studie op gang te krijgen (ere wie ere toekomt!). Stefan en Pauline, mijn kamer- en reisgenoten. Jullie zijn gezellige collega's en goede vrienden! Yvo, dank voor alle ontspanning naast het werk. Het was een luxe dat ik vaak bij jou en Lianneke doordeweeks mocht aanschuiven aan tafel. Nordin, het is geweldig dat we op dezelfde dag promoveren! Alle andere promovendi en postdocs, bedankt voor de gezellige en leerzame tijd (in alfabetische volgorde): Bas, Ben, Dionne, Eline, Elisabeth, Fleur, Frank, Jean, Jeroen, Julianne, Katrien, Kristiaan, Lian, Marja, Marnix, Nick, Olaf, Peggy, Remy, Roel, Stijn, Teba en Veronica. 
Dr. A. Koster, beste Annemarie. Bedankt dat ik van je contacten gebruik mocht maken om een stage in Amerika te regelen. Dat er nog vele gezellige etentjes met Daniella en Marieke mogen volgen!

Prof. dr. N.C. Schaper, beste Nicolaas. Jij hebt in mijn studententijd mijn eerste stappen op het wetenschappelijke vlak begeleid, en bij mijn eerste stappen nu in de kliniek voelt dat weer zo. Veel dank hiervoor.

Jos en Jeroen, de uitvoer en analyse van de vele echo-onderzoeken was (en is) een enorme klus. Zonder jullie lukt het niet om deze klus gedaan te krijgen: bedankt voor jullie onmisbare hulp.

Collega's van de afdelingen Biomedische Technologie, Fysiologie en Radiologie: prof. dr. A.P. Hoeks, prof. dr. R.S. Reneman, dr. K.D. Reesink en dr. E. Hermeling. Beste Arnold, Robert, Koen en Evelien, jullie technische kennis is cruciaal voor het slagen van de "vaattak" van De Maastricht Studie. Ik heb onze samenwerking de afgelopen jaren als zeer prettig ervaren.

Dr. A.D. Protogerou, dear Athanase. I admire your enthusiasm for science and your positive view (although the results are not always easy to grasp), and your great ability to think out of the box. I am looking forward to continue our collaboration in the future (the better navigation to "Ithaca" exists!).

Alle andere medewerkers van De Maastricht Studie en de afdeling Interne Geneeskunde: bedankt voor de zeer prettige samenwerking.

All collaborators in the Netherlands and abroad.

If I have seen further than others, it is by standing upon the shoulders of giants (Isaac Newton). During my PhD project, I was fortunate to work with top researchers in the field. Prof. dr. S. Laurent, prof. dr. P. Boutouyrie, dr. G.F. Mitchell and dr. L.J. Launer: it was a great honour for me to work with you.

I would like to express my gratitude to dr. L.J. Launer in particular. Dear Lenore, thank you for giving me the opportunity to work in your lab for several months. It was a great experience. You and all your colleagues at the National Institute on Aging (Tamara, Tad, Jennifer, Robert, Ming, Julia, Melissa, Caroline, and, last but not least, Ilse) really made me feel at home in Bethesda.

I would like to thank all other co-authors for their great inputs and collaborations: prof. dr. J.M. Dekker, prof. dr. G. Nijpels, dr. M.C. Adriaanse, dr. T. Teerlink, dr. P.G. Scheffer and prof. dr. F. Pouwer (The Hoorn Study); dr. S. Sigurdsson, prof. dr. M.A. van Buchem, dr. P.V. Jonsson, dr. V. Gudnason, dr. A.S. Levey and dr. T.B. Harris (The AGESReykjavik Study); S. Sedaghat, dr. M.A. Ikram, dr. M. Kavousi, dr. F. Mattace-Raso and prof. dr. O.H. Franco (The Rotterdam Study); prof. dr. G.M. London and dr. B. Pannier (Paris); 
and prof. dr. S. Czernichow, prof. dr. J. Blacher, prof. dr. S. Hercberg and prof. dr. B.I. Levy (The SUVIMAX2 Study).

Mijn nieuwe collega's op de afdeling Interne Geneeskunde in het ziekenhuis wil ik bedanken voor de zeer fijne samenwerking en begeleiding bij mijn eerste stappen in de kliniek.

Mijn vrienden wil ik bedanken voor de vele gezellige momenten die we hebben gehad in de afgelopen jaren. In het bijzonder Michiel, Steven, Marco, Jochem, Kees, Sinh, Cyriel en Lennart. Dat er nog vele etentjes en vakanties mogen volgen!

Als laatste, de belangrijkste mensen in mijn leven.

Pap en mam, door jullie heb ik kunnen doen wat ik graag wil. Jullie zijn mijn grootste voorbeeld. Pap, het allermooiste compliment dat ik kan krijgen, is als mensen zeggen dat ik zoveel op jou lijk. Rinske en Aart Jan, jullie zijn de beste zus en broer van de wereld. Robert en Eefje, jullie maken de familie compleet. Abel, Hanna, Rebecca en Charlotte, ik ben heel erg trots op jullie!

Theo, Loes, Jasper, Becky, bedankt dat jullie mij in jullie familie hebben opgenomen. Het voelt echt als een $2^{\mathrm{e}}$ thuis.

Marieke, het mooiste in het leven, dat ben jij! Bedankt voor alles.

Thomas 



\section{Curriculum Vitae}

Thomas Teije van Sloten was born on December 6, 1985 in The Hague, the Netherlands. He graduated from secondary school in 2004 (VWO, Zandvliet college, The Hague, cum laude). In the same year, he started his medical training at Maastricht University, and in July 2010 he obtained his medical degree. Studying medicine, he was awarded several times the top 3\% student award (academic years 2007 to 2010; grant awarded to top 3\% students of the Faculty of Health, Medicine and Life Sciences). In August 2010, he started his PhD research under supervision of Prof. Coen Stehouwer at the Department of Internal Medicine of Maastricht University Medical Centre, within the CARIM School for Cardiovascular Disease and the NUTRIM School for Nutrition, Toxicology and Metabolism. In June 2013, he obtained a Master of Health Sciences in Clinical Epidemiology at VU University, Amsterdam. During his PhD project, Thomas worked several months at the National Institutes of Health / National Institute on Aging, Bethesda, Maryland, under supervision of dr. Lenore Launer. From November 2014, Thomas has worked as a resident Internal Medicine at Maastricht University Medical Centre. 
